\title{
Development of a tagged scFv based immunoprecipitation method for protein -protein interaction studies
}

\author{
Maria Rosa Valero Aracama \\ West Virginia University
}

Follow this and additional works at: https://researchrepository.wvu.edu/etd

\section{Recommended Citation}

Valero Aracama, Maria Rosa, "Development of a tagged scFv based immunoprecipitation method for protein -protein interaction studies" (2007). Graduate Theses, Dissertations, and Problem Reports. 4346. https://researchrepository.wvu.edu/etd/4346

This Dissertation is protected by copyright and/or related rights. It has been brought to you by the The Research Repository @ WVU with permission from the rights-holder(s). You are free to use this Dissertation in any way that is permitted by the copyright and related rights legislation that applies to your use. For other uses you must obtain permission from the rights-holder(s) directly, unless additional rights are indicated by a Creative Commons license in the record and/ or on the work itself. This Dissertation has been accepted for inclusion in WVU Graduate Theses, Dissertations, and Problem Reports collection by an authorized administrator of The Research Repository @ WVU. For more information, please contact researchrepository@mail.wvu.edu. 


\title{
Development of a Tagged scFv Based Immunoprecipitation Method for Protein-protein interaction Studies
}

\author{
Maria Rosa Valero Aracama
}

Dissertation Submitted to the Eberly College of Arts and Sciences at West Virginia University in partial fulfillment of the requirements for the degree of

Doctor of Philosophy

in

Chemistry

Fred King, Ph.D., Chair

Aaron Timperman, Ph.D.

Bjorn Soderberg, Ph.D.

Patrick Callery, Ph.D.

Ronald Smart, Ph.D.

Department of Chemistry

Morgantown, West Virginia

2007 


\section{ABSTRACT \\ Development of a Tagged scFv Based Immunoprecipitation Method \\ for Protein-protein interaction Studies \\ Maria Rosa Valero Aracama}

Knowledge of the interaction between proteins is necessary for understanding how the cellular machinery operates. Multiple methods have been developed to study proteinprotein interaction, but only two have been able to provide large amount of information for S. cerevisae protein-protein network. Due to the multiple spurious data coming from these methods, there is a need of new complementary methods to improve confidence of the interactions. A new method is developed in this dissertation where the protein complexes are pulled down (immunoprecipitated) using tagged single chain antibodies specific for one of the proteins. Different affinity tags are chosen as candidates for immunoprecipitation and tested that they don't interfere with the antibody-antigen binding while remaining active and providing a clean purification in mild conditions. Strep tag II is chosen to be used as a first step in a double tag immunoprecipitation process, followed by purification using SV5 tag. Immunoprecipitation using the described method is proved in a model system. Multiple single chain antibodies are selected against proteins from a real system, Shewanella oneidensis MR-1, and checked for specificity and diversity. These single chains are used in an immunoprecipitation experiment. 


\section{Dedication:}

This dissertation is dedicated to my parents. 


\section{Acknowledgements:}

I would like to take this opportunity to thank my advisor Professor F.L. King for his guidance and support during the years of my PhD studies. His encouragement made me realize that I was capable of obtaining a $\mathrm{PhD}$ and always supported me with the decisions I took to change the course of my research. I would also like to thank Dr. Andrew Bradbury and his group at Los Alamos National Laboratory for introducing me to molecular biology and help me through out the course of my thesis. I want to thank Leslie Chasteen for being a good friend and a continuous help with my experiments and to Joanne Ayriss for being not only a great friend but for mentoring and teaching me from basic lab-book keeping to experimental planning and analysis, all with great enthusiasm and patience.

I also would like to thank to my friends in West Virginia, Carol, Nuria, Ana, Natalia, Lei and in Los Alamos, Josemari, Virginia and Priya, for all the great times we had together and all the encouragement I got in every moment of my dissertation.

But most of all I would like to thank my parents, Jose Luis and Isabel, and my brother and sisters for believing in me. Every new goal I set was received by them with great excitement and enthusiasm, never thinking that it was too high for me to reach, and enjoying with me every little success along the way.

I specially want to thank Carmen, Luca and McNair for being an example of happiness, strength and love. McNair, you helped me finish and I wish you were here to celebrate together this and many future successes to come.

I finally want to thank my husband Cris for supporting me through good and bad times, and to my two daughters, Clara and Rosa for making these years much more fun 
and being the reason to reach the end of the day with a big smile. Clara I want to thank you for making me relax after some tough days when I was trying to find a path forward on my work. Rosa I want to thank you for being a great baby while I was writing the dissertation, I don't think I could have done it without your smile from the other side of the room. 


\section{Table of Contents}

Dedication:

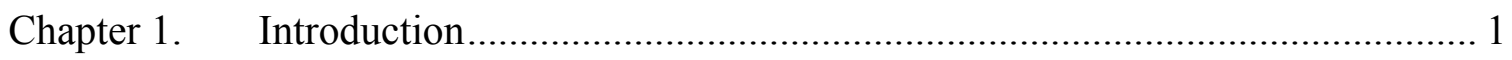

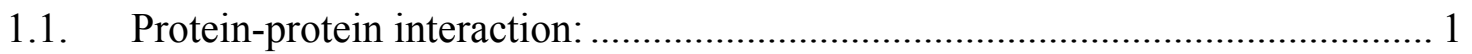

1.2 Protein-protein interaction study using single chain antibodies: ....................... 6

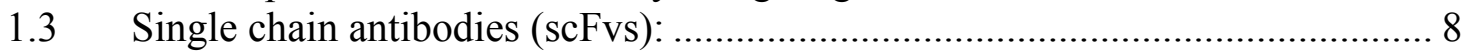

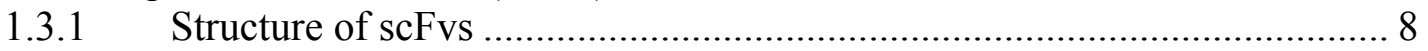

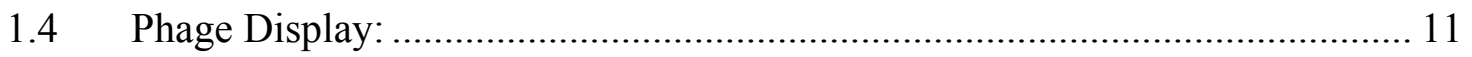

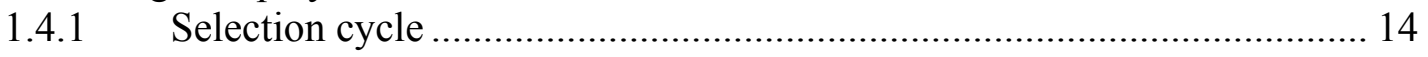

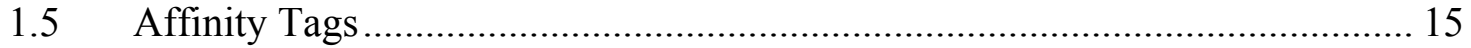

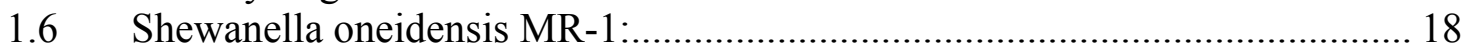

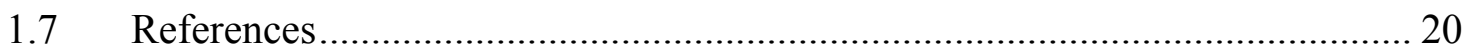

Chapter 2. Materials and Methods........................................................................ 30

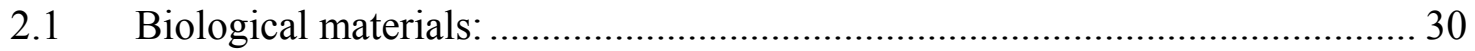

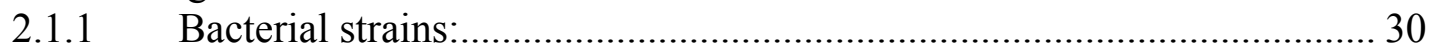

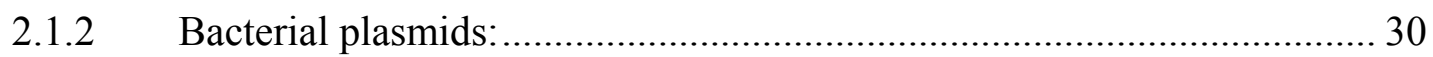

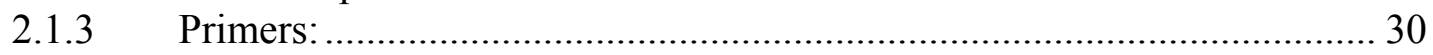

2.1.4 Primers used for tag amplification........................................................ 31

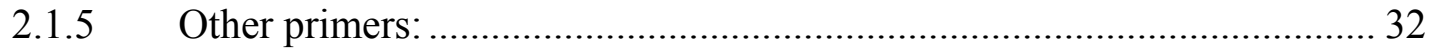

2.2 Polymerase Chain Reaction PCR: .............................................................. 32

2.3 Antibiotic concentrations: (stocks 1000x concentrated)................................... 33

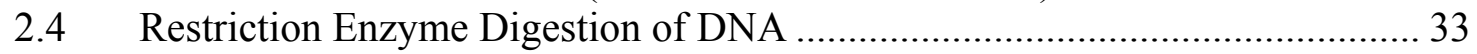

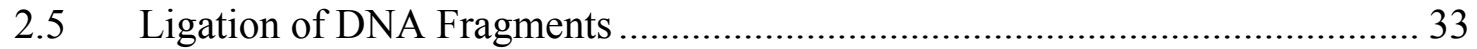

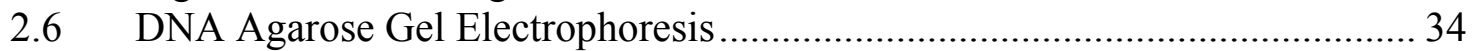

2.7 DNA recovery from agarose gel ................................................................. 35

$2.8 \quad$ Plasmid DNA preparation from Bacteria........................................................... 35

2.9 Transformation of DNA (ligation or plasmid) into competent cells................. 35

2.9.1 Electrocompetent: .............................................................................. 35

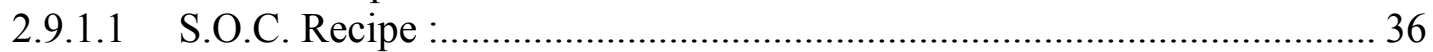

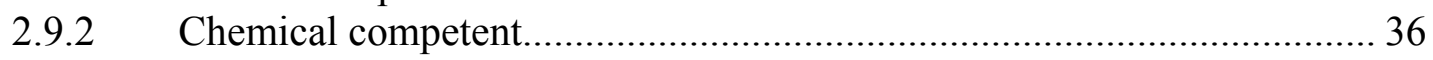

2.10 Selection of antibodies using streptavidin magnetic beads: .............................. 36

2.11 Immunotube selections: .......................................................................... 39 


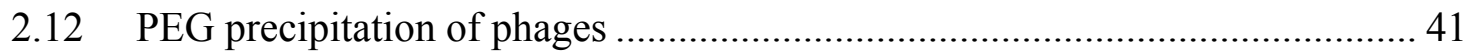

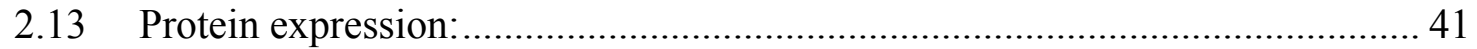

2.13.1 Expression of proteins using IPTG as inductor ...................................... 41

2.13.2 Expression of proteins using autoinducing media ................................... 42

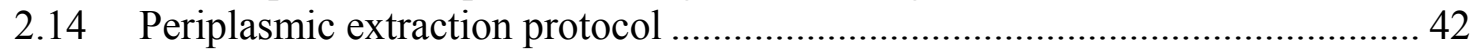

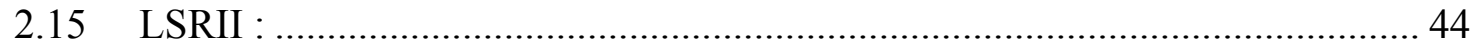

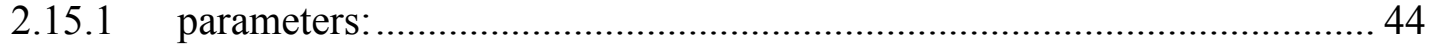

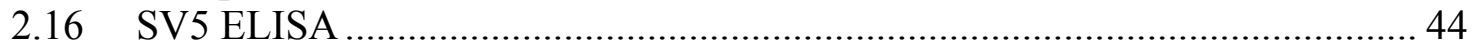

2.17 SDS polyacrylamide gel electrophoresis .................................................... 46

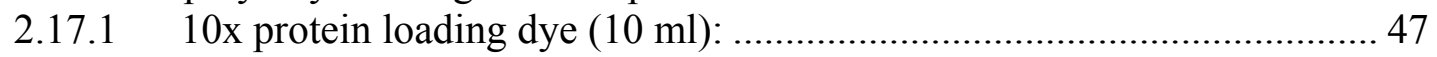

2.17.2 Gel Code staining.............................................................................. 47

2.17.3 Silver staining ................................................................................. 48

2.17.4 Gel transference into membrane (blotting): ............................................... 48

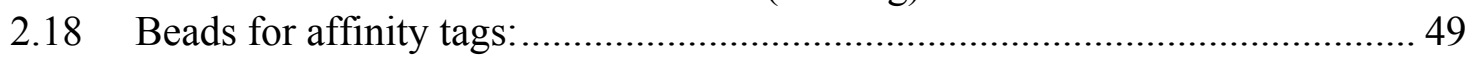

2.19 Tag purification; buffers and protocols: ..................................................... 49

2.19.1 Purification using Ni-NTA beads: ....................................................... 50

2.19.2 Purification with Strep-Tactin Beads:.................................................... 50

2.19.3 Purification with chitin Beads:................................................................ 51

2.19.4 Purification with Amylose beads:......................................................... 52

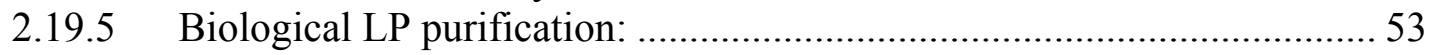

2.19.6 SV5 purification using protein A tips (Phynexus):..................................... 54

2.20 Preparation of Shewanella oneidensis MR-1 cell pellet .................................. 56

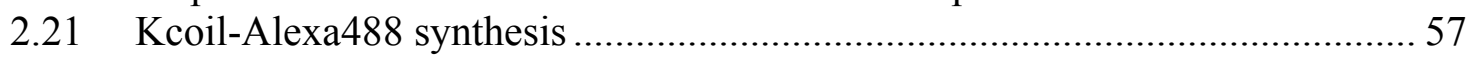

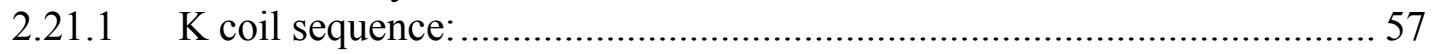

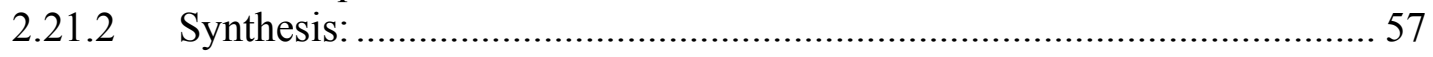

2.21.3 Deprotection/purification:................................................................... 57

2.21.4 Regeneration of the thiol after extended storage: .....................................58

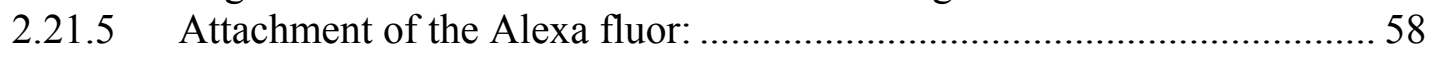

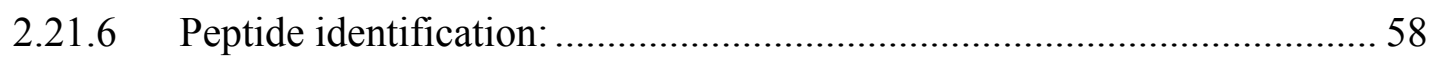

Chapter 3. Vector construction and testing ............................................................. 60

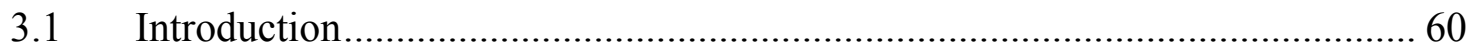

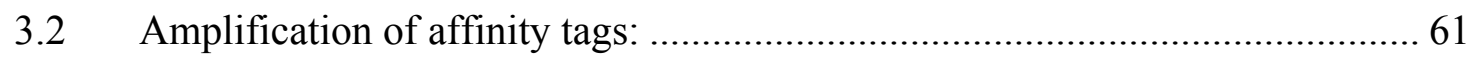

3.3 Creation of expression vector for scFv-tag fusions (pRosa6):...........................63

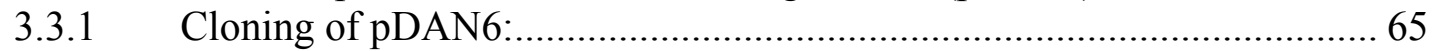

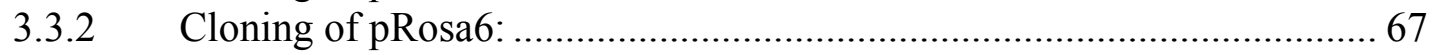

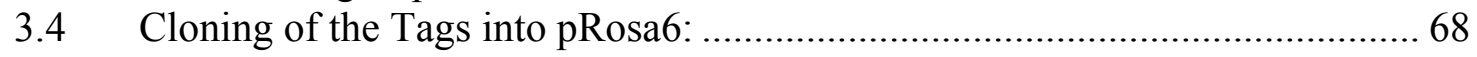

3.5 Evolution of the pRosa6 vector: ................................................................... 71

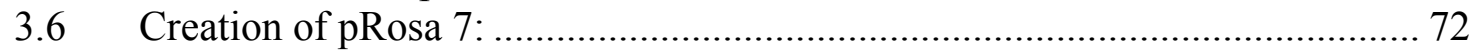

3.6.1 Cloning of scFvs into the pRosa7 vector:............................................... 76

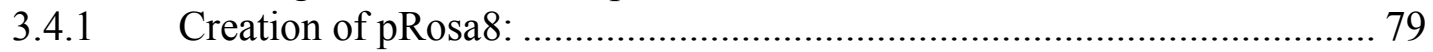

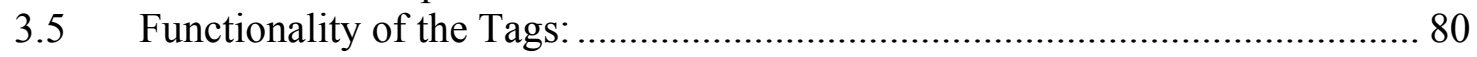

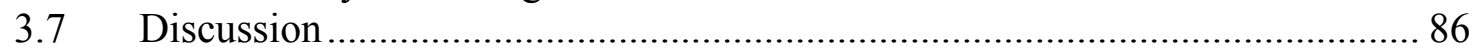

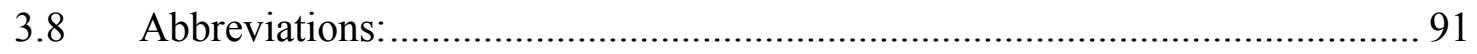

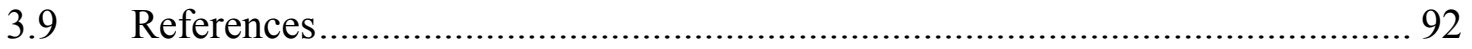

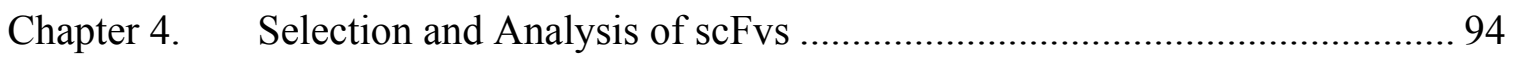




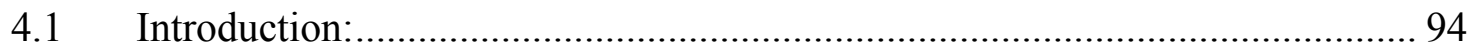

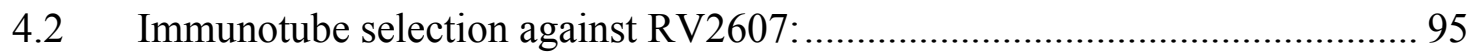

4.3 Selection against Shewanella antigens "in solution"..................................... 98

4.4 Biotinylation: ...................................................................................... 99

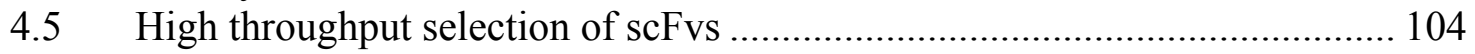

4.6 High-throughput screening of scFv ......................................................... 107

4.6.1 Labeling of the single chains (K coil-Alexa 488):................................... 108

4.6.2 Flow cytometry and ELISA analysis:.............................................. 115

4.6.3 Cloning of the scFvs into the tag vector (pRosa8-scFv-strep)................ 124

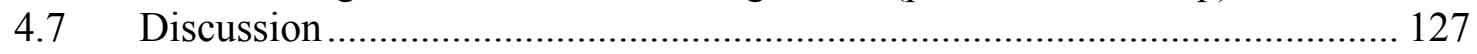

$4.8 \quad$ Appendix to Chapter 4 .............................................................................. 133

Chapter 5. Immunoprecipitation of protein complexes ............................................ 135

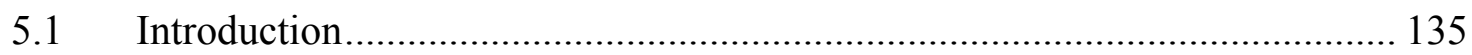

5.2 Immunoprecipitation of D1.3-lysozyme and Hyhel-10 using strep tag II and

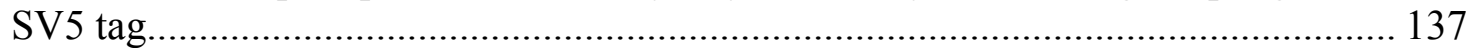

5.3 Immunoprecipitation of S.oneidensis MR-1 protein complexes .................... 141

5.3.1 Expression and purification of scFv-Tag fusions: .................................. 141

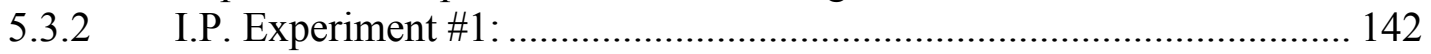

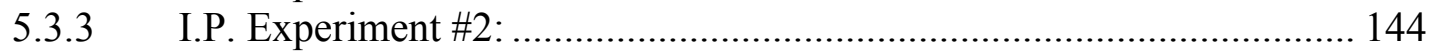

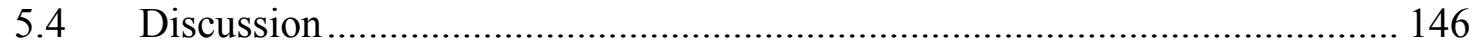

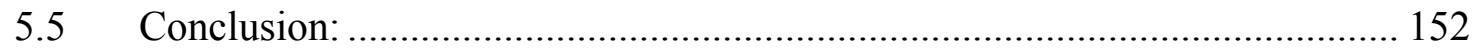

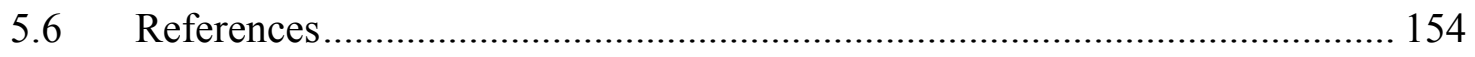

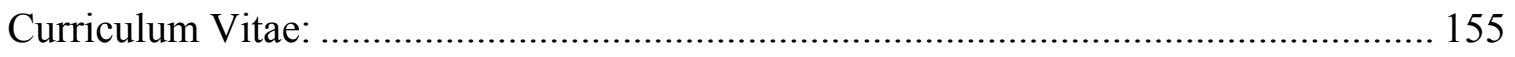




\section{List of Tables}

Table 1-1 Technical differences on the two high-throughput methods for detection of protein complexes ……................................................................................. 3

Table 1-2. Characteristics of the ideal affinity tag for immunoprecipitation purposes .... 15

Table 1-3. Characteristics of the Tags used in this study ............................................... 17

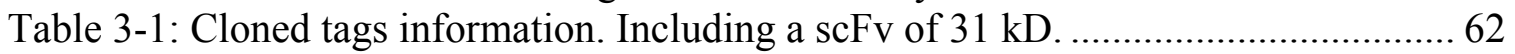

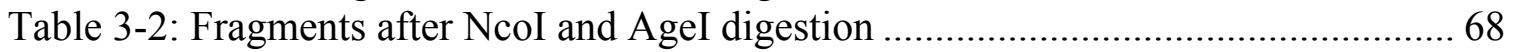

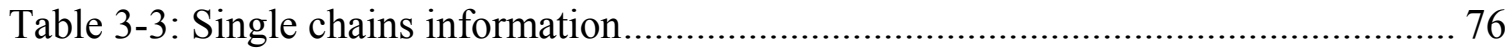

Table 3-4: bead capacity and amounts used for the experiments..................................... 81

Table 3-5: Fusions of G4 to tags and beads used to compare purifications and expected interaction with the matrices................................................................................. 82

Table 4-1. Shewanella proteins information (concentrations before dialysis) ................. 99

Table 4-2. Excess of biotin linker used for each protein .............................................. 100

Table 4-3. Antigen concentrations calculated by BCA assay......................................... 102

Table 4-4. Number of biotins per protein molecule for each of the antigens ................. 103

Table 4-5. Variation in concentration and washes between the three rounds of selection 106

Table 4-6. Ratio between numbers of colonies obtained from third round output over antigen coated beads versus the one over empty beads (negative) .......................... 106

Table 4-7: Antigens and microspheres used in flow analysis. (b- Stands for biotinylated)

Table 4-8: Analysis of binders by LSR II flow cytometec. Positive corresponds to the number of scFvs with $\mathrm{S} / \mathrm{N}$ three or larger............................................................. 118

Table 4-9: Summary of results from ELISA assays on Shewanella proteins ................. 122

Table 5-2. Components added in each of the I.P. experiments. Approximately $0.7 \mu \mathrm{g}$ of

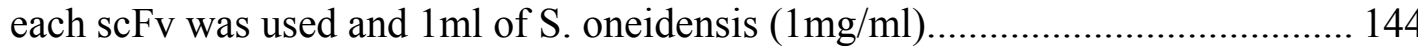




\section{List of Figures}

Figure 1-1 : General scheme of complex purification and identification in Ho and Gavin's

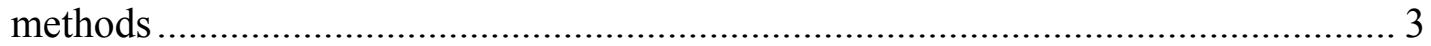

Figure 1-2. comparison of the precipitation methods in the literature and the one proposed

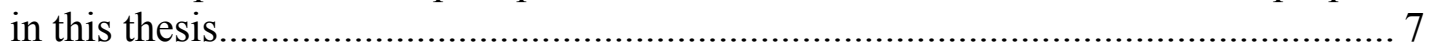

Figure 1-3. Full length antibody and detail of the binding site corresponding to the single

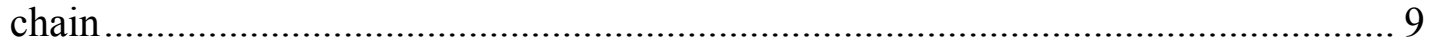

Figure 1-4. Microscopy picture of filamentous phage .................................................. 11

Figure 1-5. Phage life cycle …………………………............................................ 12

Figure 1-6:. ScFv antibody displayed on bacteriophage as a P3 fusion. .......................... 13

Figure 1-7: Phage display selection cycle.................................................................. 15

Figure 1-8. Microscopy picture of Shewanella ……….............................................. 18

Figure 3-1. Amplification of the tags by pcr; CBP, Strep-tag II, CBD, GST and MBP... 62

Figure 3-2. Schematic of the cloning process to engineer pRosa6................................... 64

Figure 3-3. Schematic of the creation of pRosa6 and fingerprint..................................... 66

Figure 3-4. Purified fragments of pET-28 (1) and pDAN6 (2) vectors, digested with

EcoRI and NcoI. Ligation of both fragments produce pRosa6................................ 67

Figure 3-5. Fingerprint of 4 colonies from pRosa6 ligation. Undigested (U), $\mathrm{NcoI}$ and EcoRI digestion (N/E), EcoRI and ClaI digestion (E/C) ........................................6 68

Figure 3-6. Digestion of pRosa6-scFv-CBP, pRosa6-scFv -chitin, pRosa6-scFv-GST, pRosa6-scFv -MBP and pRosa6-scFv -strep with NcoI and AgeI RE. .................. 69

Figure 3-7. Expression of pRosa6 vectors with different tags. Samples analyzed by SV5 Western blot and ELISA. Refer to Table 1 for sizes ............................................... 70

Figure 3-8. Evolution of the pRosa vectors ............................................................... 71

Figure 3-9. Schematic of the mutation introduced to remove the BssHII site from the lacI

gene.

Figure 3-10. BssHII digestion of pRosa7 and pRosa6................................................ 73

Figure 3-11. Diagram showing the extra R.S. found in the DNA encoding the MBP tag 73

Figure 3-12. pRosa7-scFv-chitin and $\mathrm{pRosa} 7-\mathrm{scFv}$-strep vectors checked by digestion with NcoI/EcoRI (N/E) and with BssHII (B) ................................................... 74

Figure 3-13. pRosa7-scFv-MBP vector checked by digestion with XbaI/EcoRI (X/E)... 75

Figure 3-14. Mutagenesis on the MBP gene to remove the BssHII restriction site.......... 75

Figure 3-15. BssHII digestion in mutated pRosa7-scFv-MBP (mut), and non mutated

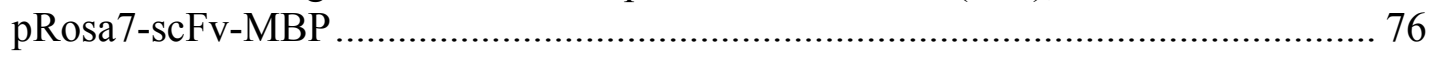

Figure 3-16. D.13 and G4 single chains digested with NheI and BssHII R.E; $\alpha$-FITC single chain digested with XbaI and NheI R.E .................................................... 77

Figure 3-17. SV5 western and ELISA assay on the tagged single chains D1.3, 4M5.3 and

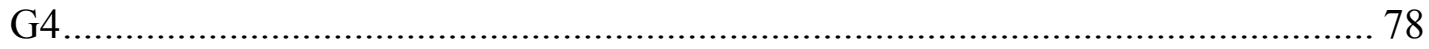

Figure 3-18. ELISA assay in D1.3-tag fusions expressed from pRosa8 vectors.............. 80

Figure 3-19. schematic of the expressed protein-tag fusions............................................ 81

Figure 3-20. Schematic of the experiment designed to check for the affinity and

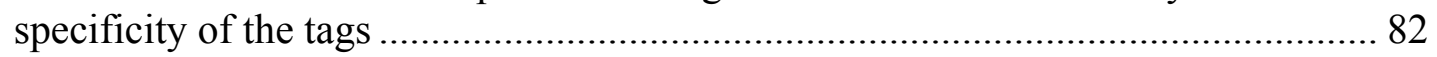

Figure 3-21. SDS-PAGE gel and SV5 western to prove the specificity of the affinity tags and the possibilities to use them as purification tags ............................................... 84 
Figure 4-1. Schematic of immunotube selection: a)Incubation of phage-scFv library with antigen coated immunotube, b) Removal of unbound phages, c) Wash in immunotube to remove non-specific binders, d) Removal of wash buffer.

Figure 4-2. $\mathrm{pDpH} 5$ '/pDpH3' amplification of ELISA positives 95

Figure 4-3. BsTN1 R.E. digestion of the full length single chains 2 different fingerprints were identified.

Figure 4-4. : SV5 westerns on clones G4 and G12 (marker: kaleidoscope Prestained Standard (BIO-RAD))

Figure 4-5. Schematic of interaction of antibodies with biotinylated antigens bound to neutravidin beads

Figure 4-6. Western blot analysis of the biotinylated antigens. Loaded approximately 200ng of each protein

Figure 4-7. SDS- PAGE analysis for protein quantification by colorimetry..... 102

Figure 4-8. Schematic of the KingFisher ${ }^{\circledR}$ working principle. (From http://www.thermo.com).

Figure 4-9 Schematic of the KingFisher ${ }^{\circledR}$ instrument and plate set up for the selection of scFvs

Figure 4-10:: Excitation of a Luminex bead with green and red lasers (Luminexcorp.com).

Figure 4-11: Coiled coils

Figure 4-12: Coil mediated labeling of single chain.

Figure 4-13: Mass spectrometric spectra of the synthesized K coil, before and after labeling.....

Figure 4-14: Western Blot Analysis of purified single chain-E/K coil fusions.............. 111

Figure 4-15: pEP-scFv-E vector diagram

Figure 4-16: BssHII/NheI digestion of scFv DNA from selections against proteins 1, $2,3,4,5$.

Figure 4-17: Fingerprint of single chains coming from selections against Shewanella antigen targets, 2, 3, 4, 5 .

Figure 4-18: Schematic of a multiplex experiment.

Figure 4-19: Data output coming from LSRII representing a positive binding event for a single chain selected against a Shewanella antigen.

Figure 4-20: Fingerprint of single chains against antigen \#4 that were positive in ELISA shown besides the corresponding ELISA and flow signals. Background is represented by an average of the signals coming from ubiquitin and neutravidin only beads. 96 clones were tested by ELISA, 46 of them also by flow.

Figure 4-21: Fingerprint of single chains against antigen \#5 that were positive in ELISA shown besides the corresponding ELISA and flow signals. Background is represented by an average of the signals coming from ubiquitin and neutravidin only beads. 96 clones were tested by ELISA, 46 of them also by flow.....

Figure 4-22: Fingerprint of positive single chains against antigen \#1 represented beside the ELISA signals. Each signal corresponds to the average of two replicates. (Neutravidin coated ELISA plate and biotinylated antigen).

Figure 4-23: ELISA against Shewanella cell extract using scFvs that were positive against antigen \#5. Each signal is the average of two replicates. 
Figure 4-24: BssHII/NheI digested fragments of pRosa8-strep vector and of single chains against antigens $\# 5$ and $\# 1$.

Figure 4-25: Alignment and phylogenetic tree of amino acid sequences of single chains selected against antigen \# 1

Figure 4-26: Alignment and phylogenetic tree of amino acid sequences of single chains selected against antigen \# 5 . ....

Figure 4-27: Raw ELISA and flow cytometry data corresponding to the scFvs selected against antigen \#4. Flow data has been represented in two different graphs in order to be able to use different scales.

Figure 4-28: Raw ELISA and flow cytometry data corresponding to the scFvs selected against antigen $\# 5$. Flow data has been represented in two different graphs in order to be able to use different scales.

Figure 5-1: Schematic of the double tag purification process.

Figure 5-2: Gel quantitation of the purified single chains. Proteins were recovered at a concentration of $0.3 \mu \mathrm{g} / \mu \mathrm{l}$ (Strep-D1.3-SV5) and $0.1 \mu \mathrm{g} / \mu \mathrm{l}$ (HyHel10-AP).

Figure 5-3: SDS Page analysis of model complex immunoprecipitation, comparison of double and single tag purifications. Left figure is the expected bands, right hand one is the actual experiment. $1 \mu \mathrm{g}$ of each scFv and equimolar amounts of lysozyme was added to $50 \mu 1$ of cell extract. Elution from strep Tactin matrix was in $100 \mu 1$ and in $30 \mu 1$ from the $\alpha$-SV5 column. Samples were loaded in equal volumes $(10 \mu 1)$. Controls of each component were added HyHel10 (1 $\mu \mathrm{g}), \alpha-\mathrm{SV} 5(2.5 \mu \mathrm{g}), \mathrm{D} 1.3(3 \mu \mathrm{g})$. Gel was developed by silver staining (chapter 2: silver staining) 139

Figure 5-4: Purified scFvs against antigens \# 5 and \#1 in Western Blot and gel code stained gel. B8 and A3 samples purified using Ni-NTA tips and all the others with agarose beads in column.

Figure 5-5: SDS PAGE gel of I.P experiments. First lane marker, second, I.P. experiment using protein \#1 and specific scFvs, third, I.P. experiment using protein \#5 and specific scFvs. $1 \mu \mathrm{g}$ of each scFv was added to $10 \mathrm{ml}$ of $\mathrm{S}$. oneidensis $(1 \mathrm{mg} / \mathrm{ml})$. Samples were incubated over night with strep Tactin beads

Figure 5-6: Analysis of the Elutions produced in I.P. experiment \#2. Figures 6A and 6B correspond to samples 1-4 analyzed by SDS PAGE and stained with either Coomassie Blue or Silver respectively. Samples 3 and 4 are those spiked with the corresponding target protein for each scFv set. Figure 6C and 6D shows analysis of samples 5 and 6 by Western Blot and silver staining respectively. Samples 5 and 6 were spiked with the corresponding non-target protein for each scFv set. 


\section{Chapter 1. Introduction}

\subsection{Protein-protein interaction:}

After completion of the Human Genome project, it was realized that the genome is composed of fewer protein-coding genes than had previously been thought ${ }^{1}{ }^{2}$. The proteome is much more dynamic than the genome, changing while it develops and due to external stimuli. Proteins form complicated interaction networks in which they regulate and support each other ${ }^{3}{ }^{56}$. Because interacting proteins are thought to be functionally related at the cellular level ${ }^{7}$, knowledge of protein complexes provides insight into the operation of the cellular machinery. Developing an improved understanding of the molecular and functional circuitry connecting protein complexes in human cells, is the basis of systems in biology and can afford benefits in medicine as well as in fundamental biomedical sciences ${ }^{8}$.

Multiple methods for protein-protein interaction identification have been developed, but the most widely used are the following:

- Co-immunoprecipitation: The most common method used to detect and purify protein-protein interactions. It is a variation of immunoprecipitation, but in this case not only the antigen binding to the antibody is identified but also other proteins interacting with it. Antibody interacts with a target antigen from a cell lysate; antigen and antibody are precipitated usually by interaction of the antibody with a protein A or G affinity matrix, washed, and eluted with any other proteins bound in complex to the antigen. 910111213

- Pull-down: Same principle as immunoprecipitation but using tagged bait proteins instead of antibodies ${ }^{14} 15$ to precipitate species of interest. 
- Yeast two hybrid: This method identifies the interaction between only two recombinant proteins at a time, expressed in yeast cells. The transcription factor is split into two fragments, a deoxyribonucleic acid (DNA) binding domain (BD) and an activation domain (AD). Pairs of proteins are expressed as fusions ('hybrids') of either of the domains. Any interaction between them leads to the formation of a functional transcription factor. ${ }^{16} 171819$

- 'In silico' (computed): Computer based interaction predictions using available experimental data and methods of prediction based on gene context analysis. $^{720}$

- Crosslinking: Proteins are covalently linked using chemical crosslinkers prior to isolation and identification of the interacting proteins ${ }^{21} 22$.

Two recent high throughput studies of multi-protein (more than two proteins) complex composition have been carried out, using co-immunoprecipitation (as shown in Figure 1.1), with the model organism Saccharomyces cerevisae by Gavin et $a^{14}$ and Ho et $a^{15}$, Both studies have provided an unprecedented amount of protein interaction information $^{23}$ using tagged S. cerevisae proteins as baits to generate complexes that are subsequently identified using mass spectrometry. The main characteristics of each coimmunoprecipitation method used by Ho and Gavin are highlighted in Table 1.1. 


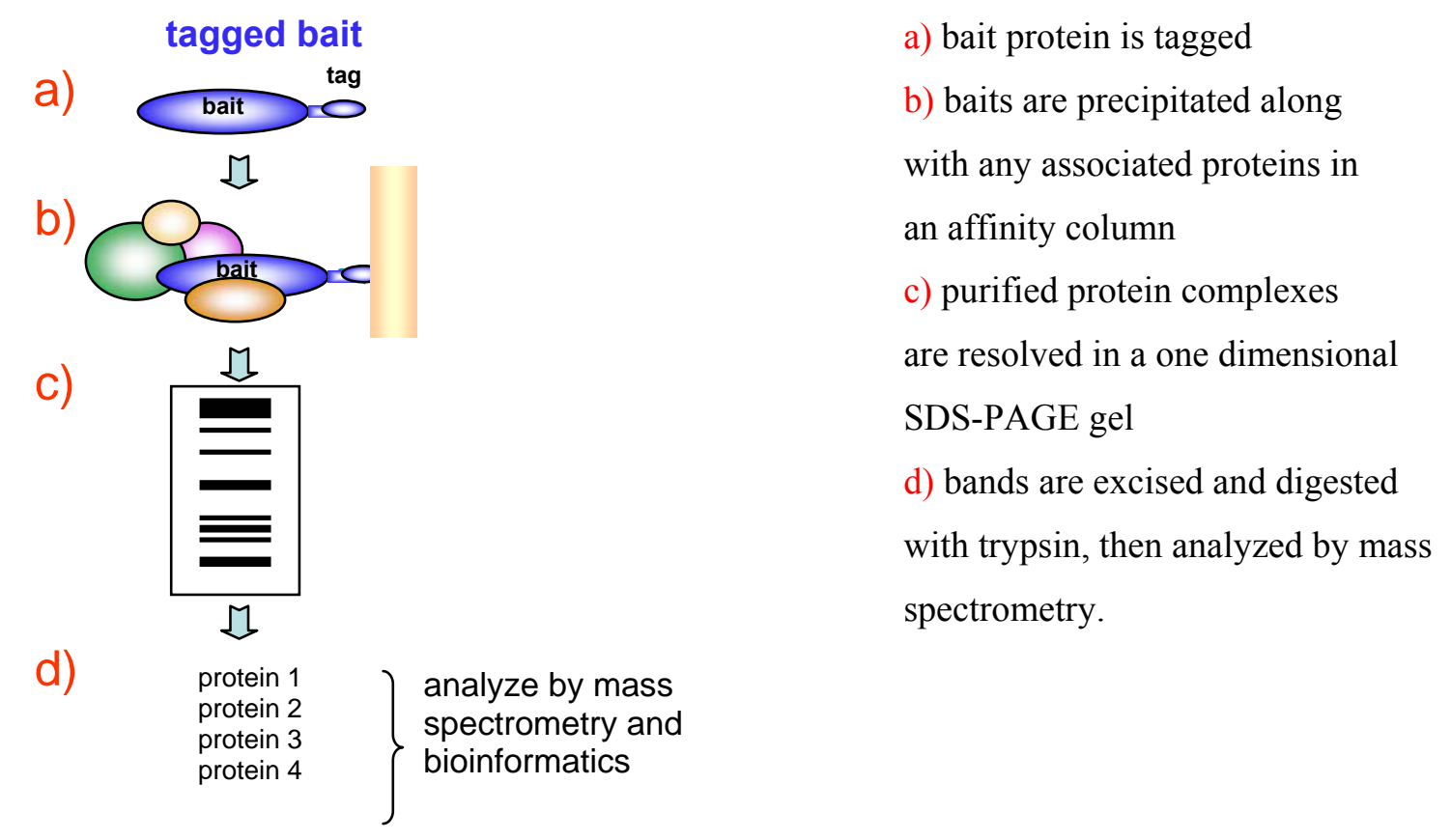

Figure 1-1 : General scheme of complex purification and identification in Ho and Gavin's methods ${ }^{24}$

\begin{tabular}{|c|c|c|}
\cline { 2 - 3 } \multicolumn{1}{c|}{} & Ho & Gavin \\
\hline bait expression & overexpressed bait proteins & wild-type expression levels \\
\hline purification & one-step purification (FLAG) & two-step purification (TAP) \\
\hline detection & LC-MS/MS & MALDI-TOF \\
\hline
\end{tabular}

Table 1-1 Technical differences on the two high-throughput methods for detection of protein complexes

Ho's method included the over-expression of bait protein to all ensure that the protein complexes were captured and available for analysis. The disadvantage is that over-expression of the bait protein could lead to the formation of artifacts because the stochiometry of the bait protein might change. As a result, it is possible that the formation of protein complexes with the bait may not occur. It is also possible that the protein interactions occurring with the bait protein are not truly representative of the complexes that are formed naturally. In an attempt to ensure bait proteins were produced with the correct stochiometry, Gavin introduced a method to include the use of an endogenous 
promoter that allowed bait proteins to be expressed at their natural level. However, the disadvantage is that if the bait protein fails to express or if the expression levels are below the critical level required for analysis, then the immunoprecitation experiment will fail.

Ho recovered the protein complexes using the FLAG ${ }^{25}$ affinity tag with a single purification step. In contrast, Gavin's method included a double purification method (Tandem Affinity Purification) ${ }^{26} 27$ using calmodulin binding peptide and protein A. Single purification allows the possibility of detecting transient protein interactions while double purification produces purer complexes with very low background. ${ }^{28}$ Because of the high degree of specificity conferred by the tandem affinity purification, stringent washes are not necessary, more effectively preserving less stable protein complexes ${ }^{29}$.

The protein complexes were detected using either liquid chromatography coupled with tandem Mass Spectrometry (Ho's method) or MALDI-TOF (Matrix-Assisted Laser Desorption/Ionization-Time-of-Flight i.e. Gavin's method).

Although both reported methods captured and identified hundreds of S. cerevisae protein complexes, the consistency of the compared data was poor. Similar inconsistencies were found when comparing results obtained using other methods such as the yeast two-hybrid technique ${ }^{18}{ }^{19}$ where only pairs of proteins, and no complexes, are identified. These inconsistencies can be explained by the fact that each method investigates different properties of the protein complexes. Therefore the data obtained is more useful as a collective resource, providing complementary information that could lead to a more complete biological overview when integrated ${ }^{8}$. Other studies, where multiple methods are compared, estimated that more than half of all current highthroughput data are spurious. This suggests that to improve confidence in detected or 
predicted protein interactions it is necessary to use as many different but complementary methods as possible. ${ }^{17}$

Both methods described by Ho and Gavin require the introduction of a genetic modification to the bait protein. The bait protein is expressed as a fusion to an unnatural protein tag in order for the protein complex to be captured for analysis. When studying protein-protein interactions it is best to form the complexes in conditions as near to their natural state as possible. Modification of the bait proteins with a tag fusion can be problematic as it is possible that the modification could affect protein conformation and ultimately generate unnatural or false protein complexes. 


\subsection{Protein-protein interaction study using single chain antibodies:}

The standard co-immunoprecipitation method (described in Figure 1.1) is an invaluable tool for the capture, detection and analysis of protein complexes. However, the method could be further improved by allowing the bait protein to remain in its natural state by eliminating the requirement to pair it with a tag for capture of the protein complex. The aim of this project is to introduce and research a new method that allows the bait protein to remain in its natural conformational state during complex formation. This proposed method includes the capture of the protein complex via a tagged single chain antibody $(\mathrm{scFv})$ that is specific for the bait protein. A schematic of the proposed method is described in Figure 1.2-1. The characteristics of this proposed method are:

- There is no genetic modification of the bait protein, as no tag is required

- Bait protein is not over expressed so real stochiometry is conserved

- Complexes are double purified providing a cleaner final product

- This method is amenable to high throughput analysis 


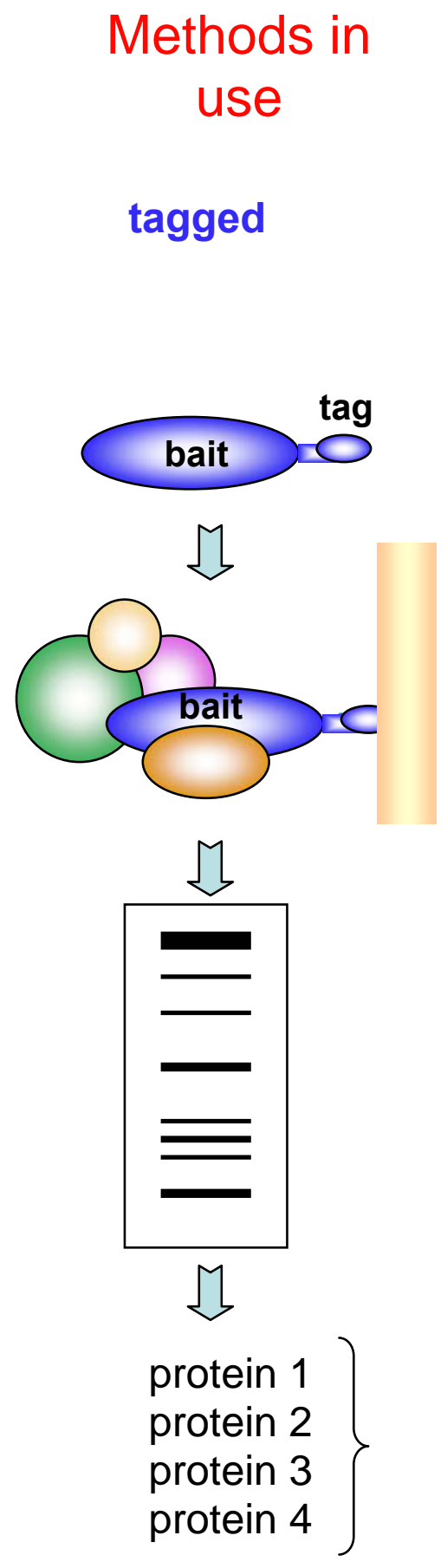

\section{Proposed method}

tagged
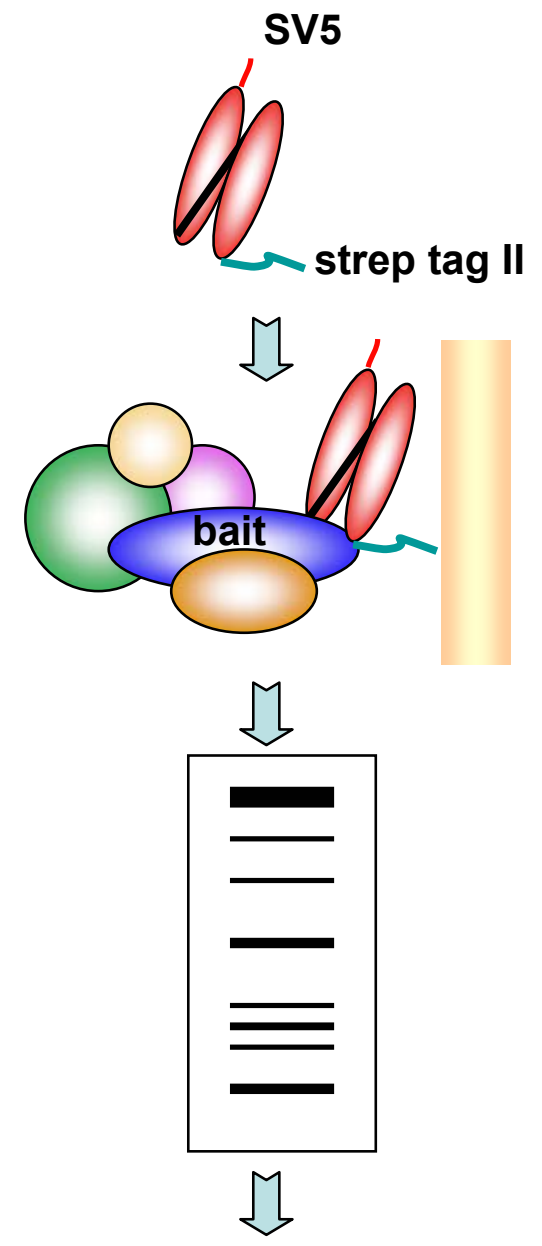

analyze by mass
spectrometry and
bioinformatics $\left\{\begin{array}{c}\text { protein } 1 \\ \text { protein } 2 \\ \text { protein } 3 \\ \text { protein } 4\end{array}\right.$

Figure 1-2. comparison of the precipitation methods in the literature and the one proposed in this thesis. 


\subsection{Single chain antibodies (scFvs):}

Antibodies are proteins that are produced by B-cells, of the immune system, in response to a target antigen (a protein or polysaccharide to which an antibody binds). Immunization of a mammalian host with target antigen stimulates an array of different Bcells to make different antibodies thus producing polyclonal antibodies. Polyclonal antibodies comprise of a mixture of multiple different antibody binders; where each different binder recognizes a different epitope on the same target antigen. The production of polyclonal sera is not reproducible even when it comes from the same animal. ${ }^{30}$ It is also a source of high background as sera contain the entire repertoire of antibodies found in the host animal at the time it was collected. ${ }^{31}$

Monoclonal antibodies are produced using hybridoma technology ${ }^{32}$. Hybridomas are generated from a single B-cell fused to a cancerous immune cell (myeloma cell). Individual hybridoma cells produce the same monoclonal antibody that recognizes the same epitope on the target antigen. Monoclonal antibodies can present problems of toxicity $^{30}$ at the high doses required to deliver sufficient antibody to the site of the tumor, can show poor penetration on tumor masses and can induce human antimouse antibody responses. $^{33}$

\subsubsection{Structure of scFvs}

Immunoglubulins are naturally occurring antibodies that are comprised of two light and two heavy polypeptide chains connected by disulfide bonds. Each of the four heavy and light chains has a constant and a variable region. The constant region provides the effector function to the antibody, while the variable region is responsible for antigen binding. ScFv antibodies are genetically engineered fusions comprising the variable 
regions of an antibody variable light-chain $\left(\mathrm{V}_{\mathrm{L}}\right)$ attached to a variable heavy chain $\left(\mathrm{V}_{\mathrm{H}}\right)$ by a polypeptide linker from the carboxyl terminus of the $\mathrm{V}_{\mathrm{L}}$ sequence to the amino terminus of the $\mathrm{V}_{\mathrm{H}}$ sequence ${ }^{46}{ }^{34}$ or vice versa $\left(\mathrm{V}_{\mathrm{H}}-\mathrm{V}_{\mathrm{L}}\right)$. The scFvs are correctly processed and are soluble ${ }^{45}$ in the oxidizing environment of the periplasm as they contain intramolecular disulfide bonds which are required for their stability.
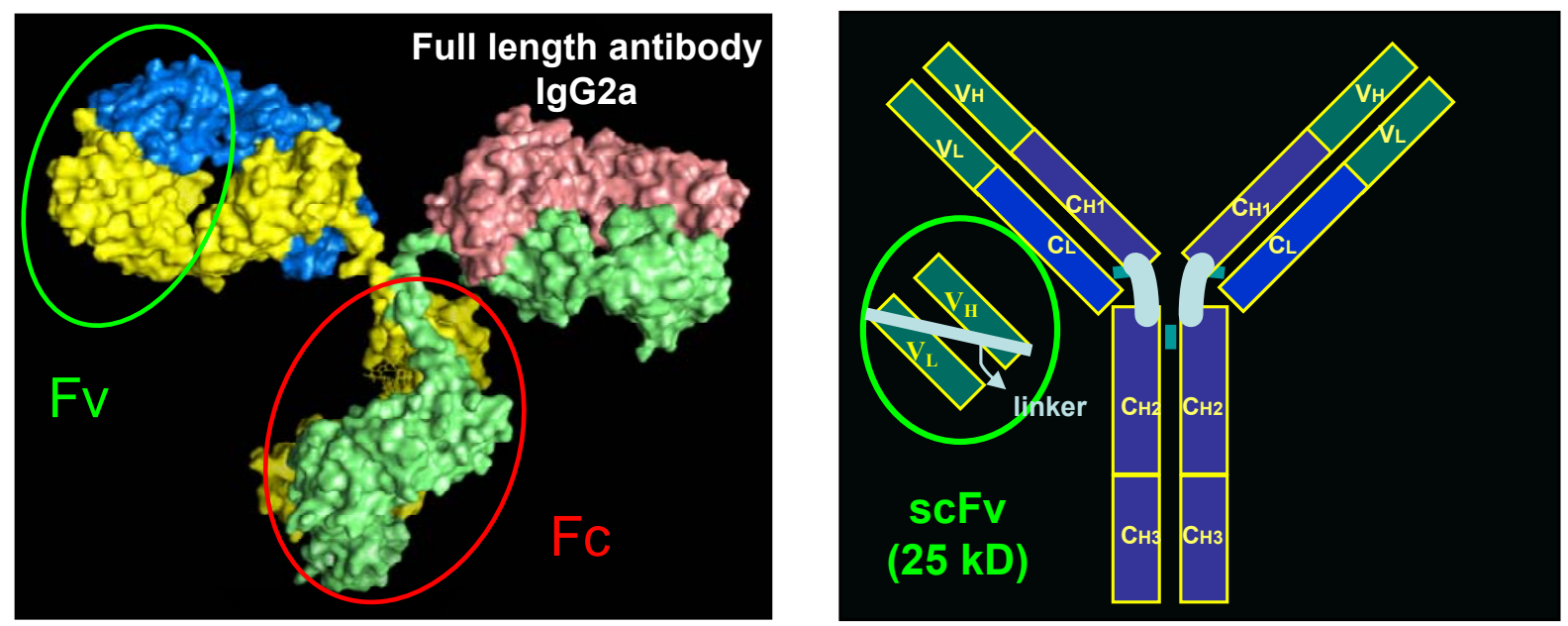

Figure 1-3. Full length antibody and detail of the binding site corresponding to the single chain

ScFvs are utilized in multiple applications including tumor targeting in vivo ${ }^{35} 36$, HIV testing of serum ${ }^{37}$, selection of antibodies against toxic antigens ${ }^{38}$, removal of toxic agents $^{34}$, antibody micro-arrays ${ }^{39}{ }^{40}$, identification of unknown cellular receptors ${ }^{41}$, detection of infection and potentially block pathogenesis ${ }^{42}$ and as a vehicle for drug delivery. ${ }^{43}$ 
Single chain antibodies (scFvs) were initially developed by two separate groups ${ }^{46}$ ${ }^{44}$ as the smallest fragments ${ }^{45}$ having the same specificities and affinities for their antigens as the parent monoclonal antibody ${ }^{46}$.

Some advantages of the use of scFvs versus the use of monoclonal antibodies are: ${ }^{4748}$

- Lower production costs.

- Higher capacity for antigen on weight basis.

- Rapid and uniform penetration in small pore matrix.

While disadvantages are:

- Decreased stability.Short half lives.

- In some cases have lower affinities to the antigen than IgGs due to the linker interfering with the binding ${ }^{49}$.

A mixture of different scFvs selected against a target antigen will produce stable multivalent interactions being equivalent to those observed by polyclonal antibodies. At the same time, background will be reduced. One of the problems with monomeric antibodies is their varied affinities for protein A; which is normally used to precipitate the complex. Using a tagged $\mathrm{scFv}$ will assure the same affinity independently of the scFv. Using pools of different scFvs in immunoprecipitation will take advantage of the properties of both polyclonal and monoclonal antibodies. 


\subsection{Phage Display:}

Filamentous bacteriophages (Genus Inovirus) are viruses that contain a circular single- stranded DNA genome encased in a long protein capsid cylinder ${ }^{50}$.

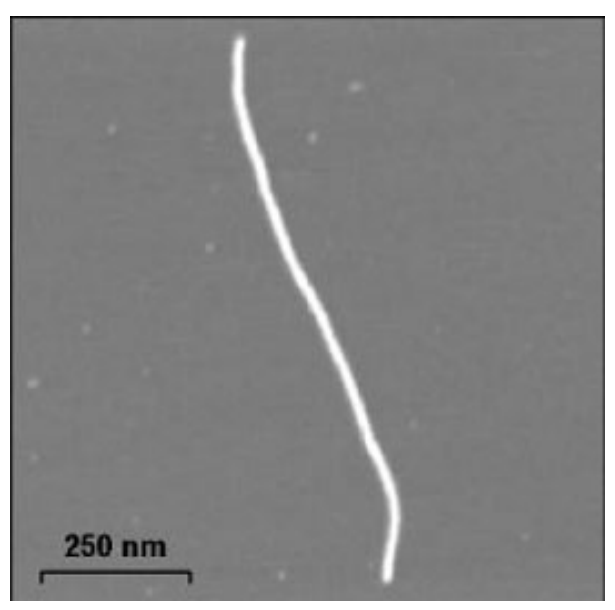

Figure 1-4. Microscopy picture of filamentous phage $\mathrm{e}^{51}$

The Ff bacteriophage infects E. coli bacteria via the F pili found in those E. coli strains that contain the F plasmid. The Ff phage does not kill the host but rather induces a state in which the infected bacterium produces and secretes phage particles ${ }^{54}$. Stages of phage life cycle: Bacteria are infected via the F pilus, viral genome is replicated, new viral particles are assembled and the progeny particles are released from the host. 


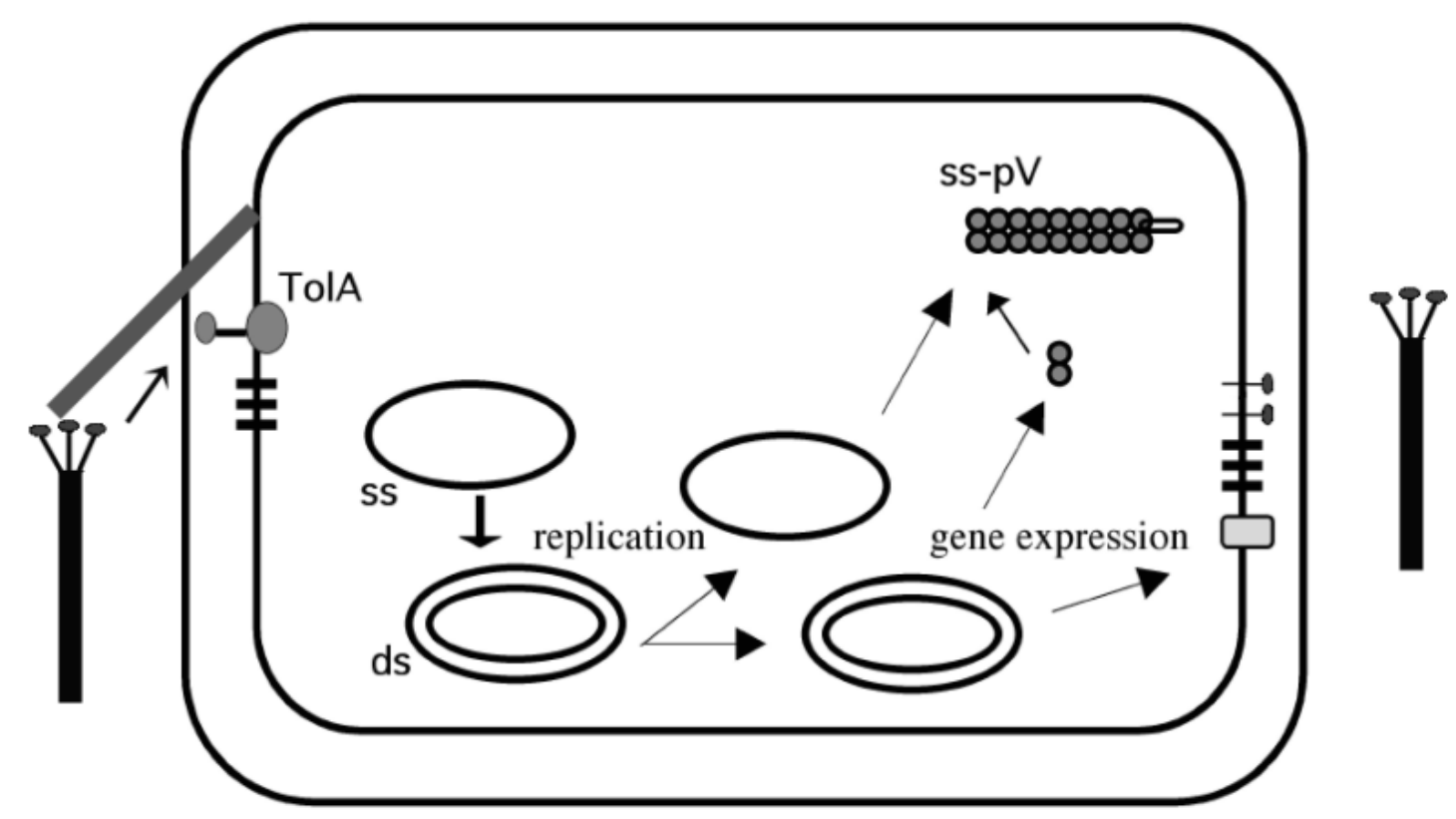

Figure 1-5. Phage life cycle ${ }^{52}$

The bacteriophage genome consists of 11 genes of which 5 encode coat proteins and the remaining six are involved in phage maturation inside the bacterial cell. The single major coat protein is encoded by gene VIII whereas 4 additional genes encode the minor coat proteins; $\mathrm{pIII}, \mathrm{pV}, \mathrm{pVII}$ and $\mathrm{pIX}$.

Smith first introduced phage display in $1985^{53}$ as a molecular diversity technology that allowed display of large protein or peptide libraries on the surface coat protein of filamentous phage ${ }^{54}$. DNA encoding the protein of interest is fused to the DNA encoding the phage-coat protein. Following infection into the host bacterial cell, the phage particle is packaged and, as a result, the protein of interest is displayed upon the surface of the bacteriophage. Out of five coat proteins, the gene 3 protein ( $\mathrm{pIII}$ or $\mathrm{p} 3$ ) is the one most commonly used for display ${ }^{55}$ being present in up to 5 copies per phage (Refer to Figure 16) 


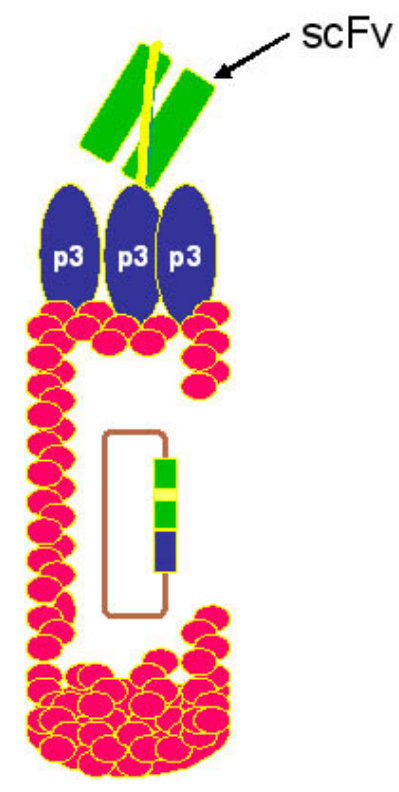

Figure 1-6:. ScFv antibody displayed on bacteriophage as a $P 3$ fusion.

The large scFv phage display library used for the work presented in this thesis was developed by Sblattero and Bradbury ${ }^{56}$. VH and VL genes were amplified by PCR from complementary DNA (cDNA) derived from human blood lymphocytes. Individual VH and VL genes were joined via a short DNA linker containing a lox recombination site using PCR primers ${ }^{57}$ and the subsequent primary library was created by cloning the assembled VH and VL fragments into the pDAN5 vector. The secondary library was created by shuffling the individual VH and VL genes by recombination and creating new $\mathrm{VH}$ and VL functional combinations. As a result of recombination, the diversity of the final tertiary scFv library is increased. In the final tertiary library, the genotype (DNA) and phenotype (displayed protein) are coupled ensuring that the gene within the phage encodes the $\mathrm{scFv}$ displayed on its surface, and providing the advantage that the $\mathrm{scFv}$ can be fused to other proteins or tags 


\subsubsection{Selection cycle}

The selection cycle is a technical process designed to recover one or more unique proteins that specifically bind to the target antigen. Specific antigen binding clones are recovered from the antibody phage library by a process called 'panning'. In this technique the phage library is incubated with a target antigen that is bound to a surface either directly or by a ligand (e.g. streptavidin) ${ }^{58} 59$. Unbound antibodies are washed off and bound ones are eluted, amplified and used as input for the subsequent selection cycle (Refer to Figure 1-7). During the panning process, the stringency of washes can be increased and the amount of target antigen decreased from one selection cycle to the next in order to enrich for the population with desired binding characteristics. Following three rounds of selection, the eluted clones are recovered and analyzed, by either ELISA or flow cytometry, for antigen binding. The aim of the panning process is to 'select' and 'enrich' for those $\mathrm{scFv}$ clones that bind specifically to the target antigen. 


\section{Phage display selection cycle}

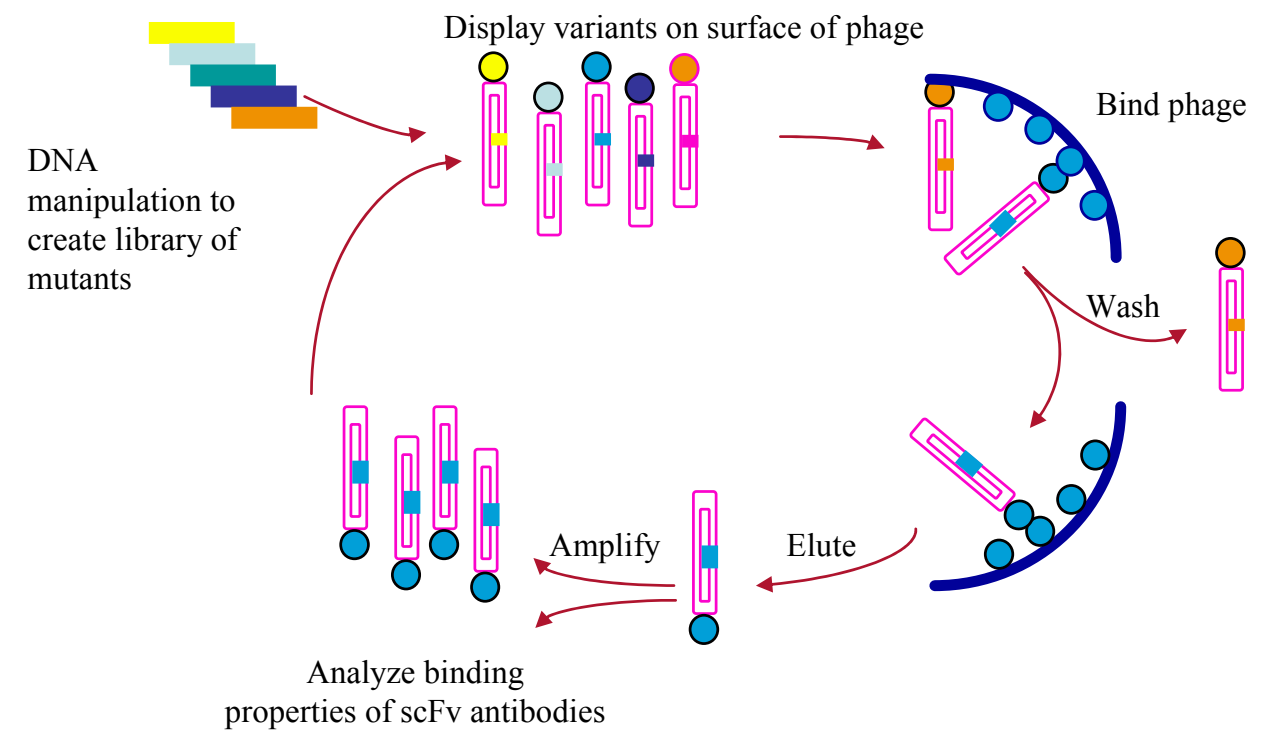

Figure 1-7: Phage display selection cycle

\subsection{Affinity Tags}

One of the aspects of this work was to study the effect of expressing scFv proteins as fusions with different protein affinity tags, the goal of which was to discover tags which could be used in immunoprecipitation. It was necessary to demonstrate that the $\mathrm{scFv}$ proteins retained their specific antigen binding properties when fused to the affinity tags, for the recovery of intact protein complexes following immuno-precipitation. Initially the tags were chosen for their biochemical properties that allowed the protein complex to be recovered by treatment with mild elution buffers.

\begin{tabular}{|c|}
\hline Properties of the ideal affinity tag for I.P. \\
\hline has small size \\
\hline is easily eluted \\
\hline has minimal effect on binding activity \\
\hline doesn't hinder or enhances expression and/or folding \\
\hline
\end{tabular}

Table 1-2. Characteristics of the ideal affinity tag for immunoprecipitation purposes 
Affinity tags are normally used to aid in the expression and purification of recombinant proteins ${ }^{60}$. There are basically two varieties of affinity tags: small peptides and larger proteins or protein domains. Small peptide tags are mainly used as purification tags whereas proteins or large peptide tags are used to enhance some characteristics of the fused protein, solubility for example. The use of a small peptide tag assures no interference with the fused protein or any other proteins that the fused protein is in complex with. Some of the most commonly used small peptide affinity tags include His $\operatorname{tag}^{61}$, myc tag ${ }^{62}$, Flag epitope ${ }^{63}$, Strep tagII ${ }^{64}$. Polyclonal and monoclonal antibodies have been raised against many of these tags, including the Flag epitope and myc tag. Because the elution conditions required to disrupt the antibody/peptide tag interaction are harsh, their use as the first step in a double purification process would not be ideal, as all the protein-protein interactions would be disrupted.

SV5 is a small tag that has been used for protein purification ${ }^{65}$ but is more generally used as a detection tag in ELISA or Western Blot assays. The SV5 tag is recognized by the monoclonal anti-SV5 $\left(\mathrm{SV} 5-\mathrm{P}-\mathrm{k}^{66}\right)$ antibody with high affinity and requires harsh buffers and denaturing conditions to disrupt the protein-protein complex for elution.

Protiens or protein domains are usually used to aid protein purification (Glutathione S-transferase $\operatorname{tag}^{67}$, protein $\mathrm{A}^{68}$, cellulose binding domains ${ }^{69}$ ), improve protein expression levels (Calmodulin-binding peptide ${ }^{70}$, chitin binding domain ${ }^{71}$ ) or increase the solubility of the fusion partner $^{72}$ (maltose binding protein $^{73}$, thioredoxin ${ }^{74}$ ). All have high specificity for their matrices. 
$\mathrm{ScFv}$ proteins are known to poorly express, have a tendency to aggregate and are not very $\mathrm{stable}^{75}$. In order to aid $\mathrm{scFv}$ production, maltose binding protein (MBP), Glutathione S-transferase tag (GST), chitin binding domain (CBD) and calmodulin binding peptide (CBP) tags were chosen as fusion tags to improve the expression and stability of the $\mathrm{scFv}$ proteins recovered in this study, in addition to allowing purification using mild elution conditions. Strep tag II was also chosen as a purification tag as it has a very high specificity for its matrix but requires mild elution conditions. It is known to produce highly pure products at moderate $\operatorname{cost}^{76}$. Due to the small size of Strep tag II $(2.5 \mathrm{kDa})$, it would not be expected to affect the functionality of the $\mathrm{scFv}$ through steric hinderence. Studies have demonstrated that the Strep tag II remains functional and does not affect the binding properties of $\mathrm{scFv}$ protein independently of the terminus that it has been fused to. ${ }^{77} 78$ Table 1-3 summarizes the main characteristics of the affinity Tags used in this study.

\begin{tabular}{|c|c|c|c|}
\hline tags & size(kD) & elution & commentaries \\
\hline MBP & 40 & maltose & enhances solubility, large \\
\hline GST & 26 & reduced glutathione & mild elution, forms dimers \\
\hline CBD & 7.7 & & cannot be eluted, good 2nd step \\
\hline CBP & 4 & EGTA & high specificity \\
\hline Strep tag II & 2.5 & biotin & high specificity \\
\hline
\end{tabular}

Table 1-3. Characteristics of the Tags used in this study

The expression vector pRosa (refer to Chapter 3) was constructed for this study to allow the Tags to be cloned either upstream (N-terminus) or downstream (C-terminus) of the $\mathrm{scFv}$ protein. All of the Tags used in this study were cloned upstream of the $\mathrm{scFv}$ protein, as $\mathrm{N}$-terminal affinity tags are known to improve the yield of recombinant protein by providing a reliable context for efficient translation initiation. ${ }^{79}$ Functional characterization of recombinant protein affinity tag fusions was required, because some 
tags have been proven to negatively affect protein function depending on the terminus to which they are fused. For example, MBP behaves a as a solubility enhancer only when it is expressed as an $\mathrm{N}$-terminal fusion. ${ }^{80}$

\subsection{Shewanella oneidensis MR-1:}

Shewanella oneidensis MR-1 (S. oneidensis) is a bacterium that can grow aerobically or anaerobically, using different electron acceptors ${ }^{81}$. This bacterium enzymatically reduces metals and radio nuclides altering their ionic properties, which affects their solubility and the solubility of other potentially harmful chemicals in their proximity. It is widely distributed in the environment and is harmless to humans or any other organism. These characteristics make $S$. oneidensis a main candidate for future bioremediation efforts. Shewanella oneidensis MR-1 was initially isolated from the anaerobic sediments of the Oneida Lake in New York State in 1988 by Myers and Nielson ${ }^{82}$ whereas other species have been isolated from marine sediments, marine waters, and a variety of other environments ${ }^{83}$.

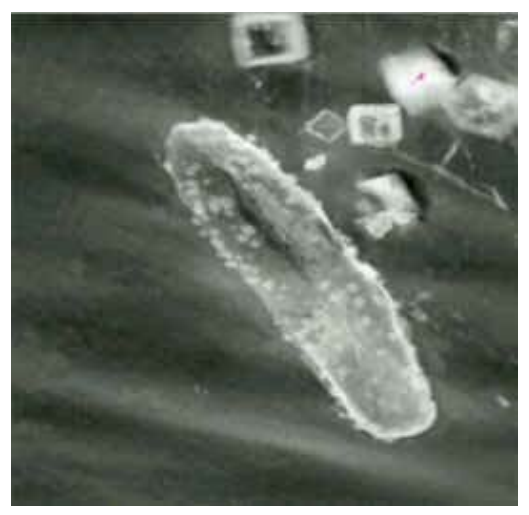

Figure 1-8. Microscopy picture of Shewanella ${ }^{84}$

The genome of Shewanella oneidensis MR-1 is comprised of approximately 5000 genes and has been recently sequenced and published ${ }^{85}$ but little is known about how it 
operates at a cellular level. A federation (Shewanella Federation) was formed to enable the sharing of information among investigators and thereby, to enable a better understanding of the microorganism at a whole-system level ${ }^{81}$.

The aim of this research project is to develop a novel immunoprecipitation method that can be used to generate and identify protein complexes. The rationale of the immunoprecipitation method is different to those currently described for multi-protein complex analysis. The method involves the generation of 'Tagged' single-chain ( $\mathrm{scFv}$ ) antibodies (using phage display) against a target antigen in order to recover a final protein complex for analysis. All components of the proposed immunoprecipitation method were tested to ensure that they were functional. A proof of principle immunoprecipitation was performed with a known antibody and antigen complex and the system was then applied to the generation and capture of S. oneidensis complexes. The proposed method can be used to generate data that compliments information generated from other IP experiments; where the bait protein is tagged and used to pull down the complexes for subsequent analysis. 


\subsection{References}

${ }^{1}$ Lander, E. S.; Linton, L. M.; Birren, B.; Nusbaum, C.; Zody, M. C.; Baldwin, J.; Devon, K.; Dewar, K.; Doyle, M.; FitzHugh, W.; Funke, R.; Gage, D.; Harris, K.; Heaford, A.; Howland, J.; Kann, L.; Lehoczky, J.; LeVine, R.; McEwan, P.; McKernan, K.; Meldrim, J.; Mesirov, J. P.; Miranda, C.; Morris, W.; Naylor, J.; Raymond, C.; Rosetti, M.; Santos, R.; Sheridan, A.; Sougnez, C.; StangeThomann, N.; Stojanovic, N.; Subramanian, A.; Wyman, D.; Rogers, J.; Sulston, J.; Ainscough, R.; Beck, S.; Bentley, D.; Burton, J.; Clee, C.; Carter, N.; Coulson, A.; Deadman, R.; Deloukas, P.; Dunham, A.; Dunham, I.; Durbin, R.; French, L.; Grafham, D.; Gregory, S.; Hubbard, T.; Humphray, S.; Hunt, A.; Jones, M.; Lloyd, C.; McMurray, A.; Matthews, L.; Mercer, S.; Milne, S.; Mullikin, J. C.; Mungall, A.; Plumb, R.; Ross, M.; Shownkeen, R.; Sims, S.; Waterston, R. H.; Wilson, R. K.; Hillier, L. W.; McPherson, J. D.; Marra, M. A.; Mardis, E. R.; Fulton, L. A.; Chinwalla, A. T.; Pepin, K. H.; Gish, W. R.; Chissoe, S. L.; Wendl, M. C.; Delehaunty, K. D.; Miner, T. L.; Delehaunty, A.; Kramer, J. B.; Cook, L. L.; Fulton, R. S.; Johnson, D. L.; Minx, P. J.; Clifton, S. W.; Hawkins, T.; Branscomb, E.; Predki, P.; Richardson, P.; Wenning, S.; Slezak, T.; Doggett, N.; Cheng, J. F.; Olsen, A.; Lucas, S.; Elkin, C.; Uberbacher, E.; Frazier, M.; Gibbs, R. A.; Muzny, D. M.; Scherer, S. E.; Bouck, J. B.; Sodergren, E. J.; Worley, K. C.; Rives, C. M.; Gorrell, J. H.; Metzker, M. L.; Naylor, S. L.; Kucherlapati, R. S.; Nelson, D. L.; Weinstock, G. M.; Sakaki, Y.; Fujiyama, A.; Hattori, M.; Yada, T.; Toyoda, A.; Itoh, T.; Kawagoe, C.; Watanabe, H.; Totoki, Y.; Taylor, T.; Weissenbach, J.; Heilig, R.; Saurin, W.; Artiguenave, F.; Brottier, P.; Bruls, T.; Pelletier, E.; Robert, C.; Wincker, P.; Smith, D. R.; Doucette-Stamm, L.; Rubenfield, M.; Weinstock, K.; Lee, H. M.; Dubois, J.; Rosenthal, A.; Platzer, M.; Nyakatura, G.; Taudien, S.; Rump, A.; Yang, H.; Yu, J.; Wang, J.; Huang, G.; Gu, J.; Hood, L.; Rowen, L.; Madan, A.; Qin, S.; Davis, R. W.; Federspiel, N. A.; Abola, A. P.; Proctor, M. J.; Myers, R. M.; Schmutz, J.; Dickson, M.; Grimwood, J.; Cox, D. R.; Olson, M. V.; Kaul, R.; Raymond, C.; Shimizu, N.; Kawasaki, K.; Minoshima, S.; Evans, G. A.; Athanasiou, M.; Schultz, R.; Roe, B. A.; Chen, F.; Pan, H.; Ramser, J.; Lehrach, H.; Reinhardt, R.; McCombie, W. R.; de la Bastide, M.; Dedhia, N.; Blocker, H.; Hornischer, K.; Nordsiek, G.; Agarwala, R.; Aravind, L.; Bailey, J. A.; Bateman, A.; Batzoglou, S.; Birney, E.; Bork, P.; Brown, D. G.; Burge, C. B.; Cerutti, L.; Chen, H. C.; Church, D.; Clamp, M.; Copley, R. R.; Doerks, T.; Eddy, S. R.; Eichler, E. E.; Furey, T. S.; Galagan, J.; Gilbert, J. G.; Harmon, C.; Hayashizaki, Y.; Haussler, D.; Hermjakob, H.; Hokamp, K.; Jang, W.; Johnson, L. S.; Jones, T. A.; Kasif, S.; Kaspryzk, A.; Kennedy, S.; Kent, W. J.; Kitts, P.; Koonin, E. V.; Korf, I.; Kulp, D.; Lancet, D.; Lowe, T. M.; McLysaght, A.; Mikkelsen, T.; Moran, J. V.; Mulder, N.; Pollara, V. J.; Ponting, C. P.; Schuler, G.; Schultz, J.; Slater, G.; Smit, A. F.; Stupka, E.; Szustakowski, J.; Thierry-Mieg, D.; Thierry-Mieg, J.; Wagner, L.; Wallis, J.; Wheeler, R.; Williams, A.; Wolf, Y. I.; Wolfe, K. H.; Yang, S. P.; Yeh, R. F.; Collins, F.; Guyer, M. S.; Peterson, J.; Felsenfeld, A.; Wetterstrand, K. A.; Patrinos, A.; Morgan, M. J.; de Jong, P.; Catanese, J. J.; Osoegawa, K.; 
Shizuya, H.; Choi, S.; Chen, Y. J., Initial sequencing and analysis of the human genome. Nature 2001, 409, (6822), 860-921.

${ }^{2}$ Venter, J. C.; Adams, M. D.; Myers, E. W.; Li, P. W.; Mural, R. J.; Sutton, G. G.; Smith, H. O.; Yandell, M.; Evans, C. A.; Holt, R. A.; Gocayne, J. D.; Amanatides, P.; Ballew, R. M.; Huson, D. H.; Wortman, J. R.; Zhang, Q.; Kodira, C. D.; Zheng, X. H.; Chen, L.; Skupski, M.; Subramanian, G.; Thomas, P. D.; Zhang, J.; Gabor Miklos, G. L.; Nelson, C.; Broder, S.; Clark, A. G.; Nadeau, J.; McKusick, V. A.; Zinder, N.; Levine, A. J.; Roberts, R. J.; Simon, M.; Slayman, C.; Hunkapiller, M.; Bolanos, R.; Delcher, A.; Dew, I.; Fasulo, D.; Flanigan, M.; Florea, L.; Halpern, A.; Hannenhalli, S.; Kravitz, S.; Levy, S.; Mobarry, C.; Reinert, K.; Remington, K.; Abu-Threideh, J.; Beasley, E.; Biddick, K.; Bonazzi, V.; Brandon, R.; Cargill, M.; Chandramouliswaran, I.; Charlab, R.; Chaturvedi, K.; Deng, Z.; Di Francesco, V.; Dunn, P.; Eilbeck, K.; Evangelista, C.; Gabrielian, A. E.; Gan, W.; Ge, W.; Gong, F.; Gu, Z.; Guan, P.; Heiman, T. J.; Higgins, M. E.; Ji, R. R.; Ke, Z.; Ketchum, K. A.; Lai, Z.; Lei, Y.; Li, Z.; Li, J.; Liang, Y.; Lin, X.; Lu, F.; Merkulov, G. V.; Milshina, N.; Moore, H. M.; Naik, A. K.; Narayan, V. A.; Neelam, B.; Nusskern, D.; Rusch, D. B.; Salzberg, S.; Shao, W.; Shue, B.; Sun, J.; Wang, Z.; Wang, A.; Wang, X.; Wang, J.; Wei, M.; Wides, R.; Xiao, C.; Yan, C.; Yao, A.; Ye, J.; Zhan, M.; Zhang, W.; Zhang, H.; Zhao, Q.; Zheng, L.; Zhong, F.; Zhong, W.; Zhu, S.; Zhao, S.; Gilbert, D.; Baumhueter, S.; Spier, G.; Carter, C.; Cravchik, A.; Woodage, T.; Ali, F.; An, H.; Awe, A.; Baldwin, D.; Baden, H.; Barnstead, M.; Barrow, I.; Beeson, K.; Busam, D.; Carver, A.; Center, A.; Cheng, M. L.; Curry, L.; Danaher, S.; Davenport, L.; Desilets, R.; Dietz, S.; Dodson, K.; Doup, L.; Ferriera, S.; Garg, N.; Gluecksmann, A.; Hart, B.; Haynes, J.; Haynes, C.; Heiner, C.; Hladun, S.; Hostin, D.; Houck, J.; Howland, T.; Ibegwam, C.; Johnson, J.; Kalush, F.; Kline, L.; Koduru, S.; Love, A.; Mann, F.; May, D.; McCawley, S.; McIntosh, T.; McMullen, I.; Moy, M.; Moy, L.; Murphy, B.; Nelson, K.; Pfannkoch, C.; Pratts, E.; Puri, V.; Qureshi, H.; Reardon, M.; Rodriguez, R.; Rogers, Y. H.; Romblad, D.; Ruhfel, B.; Scott, R.; Sitter, C.; Smallwood, M.; Stewart, E.; Strong, R.; Suh, E.; Thomas, R.; Tint, N. N.; Tse, S.; Vech, C.; Wang, G.; Wetter, J.; Williams, S.; Williams, M.; Windsor, S.; Winn-Deen, E.; Wolfe, K.; Zaveri, J.; Zaveri, K.; Abril, J. F.; Guigo, R.; Campbell, M. J.; Sjolander, K. V.; Karlak, B.; Kejariwal, A.; Mi, H.; Lazareva, B.; Hatton, T.; Narechania, A.; Diemer, K.; Muruganujan, A.; Guo, N.; Sato, S.; Bafna, V.; Istrail, S.; Lippert, R.; Schwartz, R.; Walenz, B.; Yooseph, S.; Allen, D.; Basu, A.; Baxendale, J.; Blick, L.; Caminha, M.; Carnes-Stine, J.; Caulk, P.; Chiang, Y. H.; Coyne, M.; Dahlke, C.; Mays, A.; Dombroski, M.; Donnelly, M.; Ely, D.; Esparham, S.; Fosler, C.; Gire, H.; Glanowski, S.; Glasser, K.; Glodek, A.; Gorokhov, M.; Graham, K.; Gropman, B.; Harris, M.; Heil, J.; Henderson, S.; Hoover, J.; Jennings, D.; Jordan, C.; Jordan, J.; Kasha, J.; Kagan, L.; Kraft, C.; Levitsky, A.; Lewis, M.; Liu, X.; Lopez, J.; Ma, D.; Majoros, W.; McDaniel, J.; Murphy, S.; Newman, M.; Nguyen, T.; Nguyen, N.; Nodell, M.; Pan, S.; Peck, J.; Peterson, M.; Rowe, W.; Sanders, R.; Scott, J.; Simpson, M.; Smith, T.; Sprague, A.; Stockwell, T.; Turner, R.; Venter, E.; Wang, M.; Wen, M.; Wu, D.; Wu, M.; Xia, A.; Zandieh, A.; Zhu, X., The sequence of the human genome. Science 2001, 291, (5507), 1304-51. 
${ }^{3}$ Tucker, C. L.; Gera, J. F.; Uetz, P., Towards an understanding of complex protein networks. Trends Cell Biol 2001, 11, (3), 102-6.

${ }^{4}$ Zamir, E.; Geiger, B., Molecular complexity and dynamics of cell-matrix adhesions. J Cell Sci 2001, 114, (Pt 20), 3583-90.

${ }^{5}$ Vidal, M., A biological atlas of functional maps. Cell 2001, 104, (3), 333-9.

${ }^{7}$ Marcotte, E. M.; Pellegrini, M.; Thompson, M. J.; Yeates, T. O.; Eisenberg, D., A combined algorithm for genome-wide prediction of protein function. Nature 1999, 402, (6757), 83-6.

${ }^{8}$ Gavin, A. C.; Superti-Furga, G., Protein complexes and proteome organization from yeast to man. Curr Opin Chem Biol 2003, 7, (1), 21-7.

${ }^{9}$ Birge, R. B.; Fajardo, J. E.; Reichman, C.; Shoelson, S. E.; Songyang, Z.;

Cantley, L. C.; Hanafusa, H., Identification and characterization of a high-affinity interaction between v-Crk and tyrosine-phosphorylated paxillin in CT10transformed fibroblasts. Mol Cell Biol 1993, 13, (8), 4648-56.

${ }^{10}$ Warne, P. H.; Viciana, P. R.; Downward, J., Direct interaction of Ras and the amino-terminal region of Raf-1 in vitro. Nature 1993, 364, (6435), 352-5.

${ }^{11}$ Harlow, E.; Whyte, P.; Franza, B. R., Jr.; Schley, C., Association of adenovirus early-region $1 \mathrm{~A}$ proteins with cellular polypeptides. Mol Cell Biol 1986, 6, (5), 1579-89.

${ }^{12}$ Liebler, D. C., Identifying protein-protein interactions and protein complexes. In Introduction to proteomics, tools for the new biology, Humana press: 2002; pp 151-165

${ }^{13}$ Kolodziej, P. A.; Young, R. A., Epitope tagging and protein surveillance. Methods Enzymol 1991, 194, 508-19.

${ }^{14}$ Gavin, A. C.; Bosche, M.; Krause, R.; Grandi, P.; Marzioch, M.; Bauer, A.; Schultz, J.; Rick, J. M.; Michon, A. M.; Cruciat, C. M.; Remor, M.; Hofert, C.; Schelder, M.; Brajenovic, M.; Ruffner, H.; Merino, A.; Klein, K.; Hudak, M.; Dickson, D.; Rudi, T.; Gnau, V.; Bauch, A.; Bastuck, S.; Huhse, B.; Leutwein, C.; Heurtier, M. A.; Copley, R. R.; Edelmann, A.; Querfurth, E.; Rybin, V.; Drewes, G.; Raida, M.; Bouwmeester, T.; Bork, P.; Seraphin, B.; Kuster, B.; Neubauer, G.; Superti-Furga, G., Functional organization of the yeast proteome by systematic analysis of protein complexes. Nature 2002, 415, (6868), 141-7. 
${ }^{15}$ Ho, Y.; Gruhler, A.; Heilbut, A.; Bader, G. D.; Moore, L.; Adams, S. L.; Millar, A.; Taylor, P.; Bennett, K.; Boutilier, K.; Yang, L.; Wolting, C.; Donaldson, I.; Schandorff, S.; Shewnarane, J.; Vo, M.; Taggart, J.; Goudreault, M.; Muskat, B.; Alfarano, C.; Dewar, D.; Lin, Z.; Michalickova, K.; Willems, A. R.; Sassi, H.; Nielsen, P. A.; Rasmussen, K. J.; Andersen, J. R.; Johansen, L. E.; Hansen, L. H.; Jespersen, H.; Podtelejnikov, A.; Nielsen, E.; Crawford, J.; Poulsen, V.; Sorensen, B. D.; Matthiesen, J.; Hendrickson, R. C.; Gleeson, F.; Pawson, T.; Moran, M. F.; Durocher, D.; Mann, M.; Hogue, C. W.; Figeys, D.; Tyers, M., Systematic identification of protein complexes in Saccharomyces cerevisiae by mass spectrometry. Nature 2002, 415, (6868), 180-3.

${ }^{16}$ Oliver, S., Guilt-by-association goes global. Nature 2000, 403, (6770), 601-3.

${ }^{17}$ Fields, S.; Song, O., A novel genetic system to detect protein-protein interactions. Nature 1989, 340, (6230), 245-6.

${ }^{18}$ Ito, T.; Chiba, T.; Ozawa, R.; Yoshida, M.; Hattori, M.; Sakaki, Y., A comprehensive two-hybrid analysis to explore the yeast protein interactome. Proc Natl Acad Sci U S A 2001, 98, (8), 4569-74.

${ }^{19}$ Uetz, P.; Giot, L.; Cagney, G.; Mansfield, T. A.; Judson, R. S.; Knight, J. R.; Lockshon, D.; Narayan, V.; Srinivasan, M.; Pochart, P.; Qureshi-Emili, A.; Li, Y.; Godwin, B.; Conover, D.; Kalbfleisch, T.; Vijayadamodar, G.; Yang, M.; Johnston, M.; Fields, S.; Rothberg, J. M., A comprehensive analysis of protein-protein interactions in Saccharomyces cerevisiae. Nature 2000, 403, (6770), 623-7.

${ }^{20}$ Enright, A. J.; Iliopoulos, I.; Kyrpides, N. C.; Ouzounis, C. A., Protein interaction maps for complete genomes based on gene fusion events. Nature 1999, 402, (6757), 86-90.

${ }^{21}$ Ishiguro, A.; Kimura, M.; Yasui, K.; Iwata, A.; Ueda, S.; Ishihama, A., Two large subunits of the fission yeast RNA polymerase II provide platforms for the assembly of small subunits. J Mol Biol 1998, 279, (4), 703-12.

${ }^{22}$ Mullins, R. D.; Stafford, W. F.; Pollard, T. D., Structure, subunit topology, and actin-binding activity of the Arp2/3 complex from Acanthamoeba. J Cell Biol 1997, 136, (2), 331-43.

${ }^{23}$ Bader GD, H. C., Analyzing yeast protein-protein interaction data obtained from different sources. Nat Biotechnol. 2002, 20, (10), 991-997.

${ }^{24}$ Kumar, A.; Snyder, M., Protein complexes take the bait. Nature 2002, 415, (6868), 123-4. 
${ }^{25}$ Knappik, A.; Pluckthun, A., An improved affinity tag based on the FLAG peptide for the detection and purification of recombinant antibody fragments. Biotechniques 1994, 17, (4), 754-61.

${ }^{26}$ Rigaut, G.; Shevchenko, A.; Rutz, B.; Wilm, M.; Mann, M.; Seraphin, B., A generic protein purification method for protein complex characterization and proteome exploration. Nat Biotechnol 1999, 17, (10), 1030-2.

${ }^{27}$ Puig, O.; Caspary, F.; Rigaut, G.; Rutz, B.; Bouveret, E.; Bragado-Nilsson, E.; Wilm, M.; Seraphin, B., The tandem affinity purification (TAP) method: a general procedure of protein complex purification. Methods 2001, 24, (3), 218-29.

${ }^{28}$ von Mering, C.; Krause, R.; Snel, B.; Cornell, M.; Oliver, S. G.; Fields, S.; Bork, $P$. ., Comparative assessment of large-scale data sets of protein-protein interactions. Nature 2002, 417, (6887), 399-403.

${ }^{29}$ Gingras A., Aebersold R., Raught B., Advances in protein complex analysis using mass spectrometry. J. Physiol. 2005, 563, 1, 11-21

${ }^{30}$ Bradbury, A.; Velappan, N.; Verzillo, V.; Ovecka, M.; Chasteen, L.; Sblattero, D.; Marzari, R.; Lou, J.; Siegel, R.; Pavlik, P., Antibodies in proteomics I: generating antibodies. Trends Biotechnol 2003, 21, (6), 275-81.

${ }^{31}$ Molecular cloning book (red one)

${ }^{32}$ Kohler, G.; Milstein, C., Continuous cultures of fused cells secreting antibody of predefined specificity. Nature 1975, 256, (5517), 495-7.

${ }^{33}$ Myers, K.; R., M; Stern, P; Shaw, D; Embleton, J; Kingsman, S; Carroll, M Targeting immune effector molecules to human tumor cells through genetic delivery of 5T4-specific scFv fusion proteins. Cancer Gene Therapy 2002, 9, (11), 884-896.

${ }^{34}$ Huston, J. S.; Levinson, D.; Mudgett-Hunter, M.; Tai, M. S.; Novotny, J.; Margolies, M. N.; Ridge, R. J.; Bruccoleri, R. E.; Haber, E.; Crea, R.; et al., Protein engineering of antibody binding sites: recovery of specific activity in an anti-digoxin single-chain Fv analogue produced in Escherichia coli. Proc Natl Acad Sci U S A 1988, 85, (16), 5879-83.

${ }^{35}$ Huston, J. S.; McCartney, J.; Tai, M. S.; Mottola-Hartshorn, C.; Jin, D.; Warren, F.; Keck, P.; Oppermann, H., Medical applications of single-chain antibodies. Int Rev Immunol 1993, 10, (2-3), 195-217.

${ }^{36}$ Hanisch, F. G.; Uhlenbruck, G.; Egge, H.; Peter-Katalinic, J., A B72.3 secondgeneration-monoclonal antibody (CC49) defines the mucin-carried carbohydrate 
epitope Gal beta(1-3) [NeuAc alpha(2-6)]GalNAc. Biol Chem Hoppe Seyler 1989, 370, (1), 21-6.

${ }^{37}$ Lilley, G. G.; Dolezal, O.; Hillyard, C. J.; Bernard, C.; Hudson, P. J., Recombinant single-chain antibody peptide conjugates expressed in Escherichia coli for the rapid diagnosis of HIV. J Immunol Methods 1994, 171, (2), 211-26.

${ }^{38}$ Vaughan, T. J.; Williams, A. J.; Pritchard, K.; Osbourn, J. K.; Pope, A. R.; Earnshaw, J. C.; McCafferty, J.; Hodits, R. A.; Wilton, J.; Johnson, K. S., Human antibodies with sub-nanomolar affinities isolated from a large non-immunized phage display library. Nat Biotechnol 1996, 14, (3), 309-14.

${ }^{39}$ Kusnezow, W.; Syagailo, Y. V.; Goychuk, I.; Hoheisel, J. D.; Wild, D. G., Antibody microarrays: the crucial impact of mass transport on assay kinetics and sensitivity. Expert Rev Mol Diagn 2006, 6, (1), 111-24.

${ }^{40}$ Kusnezow, W.; Syagailo, Y. V.; Ruffer, S.; Klenin, K.; Sebald, W.; Hoheisel, J. D.; Gauer, C.; Goychuk, I., Kinetics of antigen binding to antibody microspots: strong limitation by mass transport to the surface. Proteomics 2006, 6, (3), 794803.

${ }^{41}$ Wang, B.; Chen, Y. B.; Ayalon, O.; Bender, J.; Garen, A., Human single-chain Fv immunoconjugates targeted to a melanoma-associated chondroitin sulfate proteoglycan mediate specific lysis of human melanoma cells by natural killer cells and complement. Proc Natl Acad Sci U S A 1999, 96, (4), 1627-32.

${ }^{42}$ Cirino, N. M.; Sblattero, D.; Allen, D.; Peterson, S. R.; Marks, J. D.; Jackson, P. J.; Bradbury, A.; Lehnert, B. E., Disruption of anthrax toxin binding with the use of human antibodies and competitive inhibitors. Infect Immun 1999, 67, (6), 295763.

${ }^{43}$ Sun, C.; Wirsching, P.; Janda, K. D., Enabling ScFvs as multi-drug carriers: a dendritic approach. Bioorg Med Chem 2003, 11, (8), 1761-8.

${ }^{44}$ Huston, J. S.; Levinson, D.; Mudgett-Hunter, M.; Tai, M. S.; Novotny, J.; Margolies, M. N.; Ridge, R. J.; Bruccoleri, R. E.; Haber, E.; Crea, R.; et al., Protein engineering of antibody binding sites: recovery of specific activity in an anti-digoxin single-chain Fv analogue produced in Escherichia coli. Proc Natl Acad Sci U S A 1988, 85, (16), 5879-83.

${ }^{45}$ Glockshuber, R.; Malia, M.; Pfitzinger, I.; Pluckthun, A., A comparison of strategies to stabilize immunoglobulin Fv-fragments. Biochemistry 1990, 29, (6), 1362-7. 
${ }^{46}$ Bird, R. E.; Hardman, K. D.; Jacobson, J. W.; Johnson, S.; Kaufman, B. M.; Lee, S. M.; Lee, T.; Pope, S. H.; Riordan, G. S.; Whitlow, M., Single-chain antigen-binding proteins. Science 1988, 242, (4877), 423-6.

${ }^{47}$ Kipriyanov, S. M.; Little, M., Affinity purification of tagged recombinant proteins using immobilized single chain Fv fragments. Anal Biochem 1997, 244, (1), 18991.

${ }^{48}$ Leath, C. A., 3rd; Douglas, J. T.; Curiel, D. T.; Alvarez, R. D., Single-chain antibodies: A therapeutic modality for cancer gene therapy (review). Int J Oncol 2004, 24, (4), 765-71.

${ }^{49}$ Gao, C. M., S.; Lo, C.; Wirsching, P.; Lerner, R.; Janda, K., Making artificial antibodies: A format for phage display heterodimeric arrays. Proc Natl Acad Sci U S A 1999, 96, (11), 6025- 6030.

${ }^{50}$ Barbas, C. B., D.; Scott, J; Silverman, G., Phage Display, A Laboratory Manual. 2001.

${ }^{51} \mathrm{Ji}, \mathrm{X} . ;$ Oh, J.; Dunker, A. K.; Hipps, K. W., Effects of relative humidity and applied force on atomic force microscopy images of the filamentous phage fd. Ultramicroscopy 1998, 72, (3-4), 165-76.

${ }^{52}$ Abedon, S. T.; The Bacteriophages, $2^{\text {nd }}$ edition(2004)

${ }^{53}$ Smith, G. P., Filamentous fusion phage: novel expression vectors that display cloned antigens on the virion surface. Science 1985, 228, (4705), 1315-7.

${ }^{54}$ Azzazy, H. M.; Highsmith, W. E., Jr., Phage display technology: clinical applications and recent innovations. Clin Biochem 2002, 35, (6), 425-45.

${ }^{55}$ Chasteen, L.; Ayriss, J.; Pavlik, P.; Bradbury, A. R., Eliminating helper phage from phage display. Nucleic Acids Res 2006, 34, (21), e145.

${ }^{56}$ Sblattero, D.; Bradbury, A., Exploiting recombination in single bacteria to make large phage antibody libraries. Nat Biotechnol 2000, 18, (1), 75-80.

${ }^{57}$ Krebber, A.; Bornhauser, S.; Burmester, J.; Honegger, A.; Willuda, J.; Bosshard, H. R.; Pluckthun, A., Reliable cloning of functional antibody variable domains from hybridomas and spleen cell repertoires employing a reengineered phage display system. J Immunol Methods 1997, 201, (1), 35-55.

${ }^{58}$ Clackson, T.; Hoogenboom, H. R.; Griffiths, A. D.; Winter, G., Making antibody fragments using phage display libraries. Nature 1991, 352, (6336), 624-8. 
${ }^{59}$ Nissim, A.; Hoogenboom, H. R.; Tomlinson, I. M.; Flynn, G.; Midgley, C.; Lane, D.; Winter, G., Antibody fragments from a 'single pot' phage display library as immunochemical reagents. Embo J 1994, 13, (3), 692-8.

${ }^{60}$ Terpe, K., Overview of tag protein fusions: from molecular and biochemical fundamentals to commercial systems. Appl Microbiol Biotechnol 2003, 60, (5), 523-33.

${ }^{61}$ Hochuli, E. B., W; Dobeli, H; Gentz, R; Stuber, D, Genetic approach to facilitate purification of recombinant proteins with a novel metal chelate adsorbent. Bio/Technology 1988, 6, 1321-1325.

${ }^{62}$ Munro, S.; Pelham, H. R., An Hsp70-like protein in the ER: identity with the 78 kd glucose-regulated protein and immunoglobulin heavy chain binding protein. Cell 1986, 46, (2), 291-300.

${ }^{63}$ Hopp, T. P., KS; Price, VL; Libby, RT; March, CJ; Ceretti, DP; Urdal, DL; Conlon, $\mathrm{PJ}$, A short polypeptide marker sequence useful for recombinant protein identification and purification Bio/Technology 1988, 6, 1204-1210.

${ }^{64}$ Schmidt, T. G.; Skerra, A., The random peptide library-assisted engineering of a C-terminal affinity peptide, useful for the detection and purification of a functional Ig Fv fragment. Protein Eng 1993, 6, (1), 109-22.

${ }^{65}$ Randall, R. E.; Hanke, T.; Young, D.; Southern, J. A., Two-tag purification of recombinant proteins for the construction of solid matrix-antibody-antigen (SMAA) complexes as vaccines. Vaccine 1993, 11, (12), 1247-52.

${ }^{66}$ Hanke, T.; Randall, R. E., Variable domain sequences of mAb with high affinity for a linear oligopeptide. Immunogenetics 1995, 42, (5), 442-3.

${ }^{67}$ Smith, D. B.; Johnson, K. S., Single-step purification of polypeptides expressed in Escherichia coli as fusions with glutathione S-transferase. Gene 1988, 67, (1), 31-40.

${ }^{68}$ Nilsson, B.; Abrahmsen, L.; Uhlen, M., Immobilization and purification of enzymes with staphylococcal protein A gene fusion vectors. Embo J 1985, 4, (4), 1075-80.

${ }^{69}$ Xu, Z.; Bae, W.; Mulchandani, A.; Mehra, R. K.; Chen, W., Heavy metal removal by novel CBD-EC20 sorbents immobilized on cellulose.

Biomacromolecules 2002, 3, (3), 462-5. 
${ }^{70}$ Zheng, C. F.; Simcox, T.; Xu, L.; Vaillancourt, P., A new expression vector for high level protein production, one step purification and direct isotopic labeling of calmodulin-binding peptide fusion proteins. Gene 1997, 186, (1), 55-60.

${ }^{71}$ Watanabe, T.; Ito, Y.; Yamada, T.; Hashimoto, M.; Sekine, S.; Tanaka, H., The roles of the C-terminal domain and type III domains of chitinase A1 from Bacillus circulans WL-12 in chitin degradation. J Bacteriol 1994, 176, (15), 4465-72.

${ }^{72}$ Baneyx, F., Recombinant protein expression in Escherichia coli. Curr Opin Biotechnol 1999, 10, (5), 411-21.

${ }^{73}$ di Guan, C.; Li, P.; Riggs, P. D.; Inouye, H., Vectors that facilitate the expression and purification of foreign peptides in Escherichia coli by fusion to maltose-binding protein. Gene 1988, 67, (1), 21-30.

${ }^{74}$ LaVallie, E. R.; Lu, Z.; Diblasio-Smith, E. A.; Collins-Racie, L. A.; McCoy, J. M., Thioredoxin as a fusion partner for production of soluble recombinant proteins in Escherichia coli. Methods Enzymol 2000, 326, 322-40.

${ }^{75}$ Bradbury, A. R.; Marks, J. D., Antibodies from phage antibody libraries. J Immunol Methods 2004, 290, (1-2), 29-49.

${ }^{76}$ Lichty, J. J.; Malecki, J. L.; Agnew, H. D.; Michelson-Horowitz, D. J.; Tan, S., Comparison of affinity tags for protein purification. Protein Expr Purif 2005, 41, (1), 98-105.

${ }^{77}$ Aubrey, N.; Devaux, C.; di Luccio, E.; Goyffon, M.; Rochat, H.; Billiald, P., A recombinant scFv/streptavidin-binding peptide fusion protein for the quantitative determination of the scorpion venom neurotoxin Aahl. Biol Chem 2001, 382, (11), 1621-8.

${ }^{78}$ Kolln, J.; Spillner, E.; Andra, J.; Klensang, K.; Bredehorst, R., Complement inactivation by recombinant human C3 derivatives. J Immunol 2004, 173, (9), 5540-5.

${ }^{79}$ Waugh, D. S., Making the most of affinity tags. Trends Biotechnol 2005, 23, (6), 316-20.

${ }^{80}$ Sachdev, D.; Chirgwin, J. M., Order of fusions between bacterial and mammalian proteins can determine solubility in Escherichia coli. Biochem Biophys Res Commun 1998, 244, (3), 933-7.

${ }^{81} \mathrm{http}: / / \mathrm{www}$. shewanella.org/ 
${ }^{82}$ Myers, C, Nealson, K, Bacterial manganese reduction and growth with manganese oxide as the sole electron acceptor, Science, 1988, 1319-1321

${ }^{83}$ Nealson, K. H., and Scott, J. (2003) in The Prokaryotes: An Evolving Electronic Resource for the Microbiological Community (Dworkin, M., ed) 3rd Ed., SpringerVerlag Inc., New York

${ }^{84}$ http://genomicsgtl.energy.gov/research/shewanella/JIM files/frame.htm

${ }^{85}$ Heidelberg, J. F.; Paulsen, I. T.; Nelson, K. E.; Gaidos, E. J.; Nelson, W. C.; Read, T. D.; Eisen, J. A.; Seshadri, R.; Ward, N.; Methe, B.; Clayton, R. A.; Meyer, T.; Tsapin, A.; Scott, J.; Beanan, M.; Brinkac, L.; Daugherty, S.; DeBoy, R. T.; Dodson, R. J.; Durkin, A. S.; Haft, D. H.; Kolonay, J. F.; Madupu, R.; Peterson, J. D.; Umayam, L. A.; White, O.; Wolf, A. M.; Vamathevan, J.; Weidman, J.; Impraim, M.; Lee, K.; Berry, K.; Lee, C.; Mueller, J.; Khouri, H.; Gill, J.; Utterback, T. R.; McDonald, L. A.; Feldblyum, T. V.; Smith, H. O.; Venter, J. C.; Nealson, K. H.; Fraser, C. M., Genome sequence of the dissimilatory metal ion-reducing bacterium Shewanella oneidensis. Nat Biotechnol 2002, 20, (11), 1118-23. 


\section{Chapter 2. Materials and Methods}

\subsection{Biological materials:}

\subsubsection{Bacterial strains:}

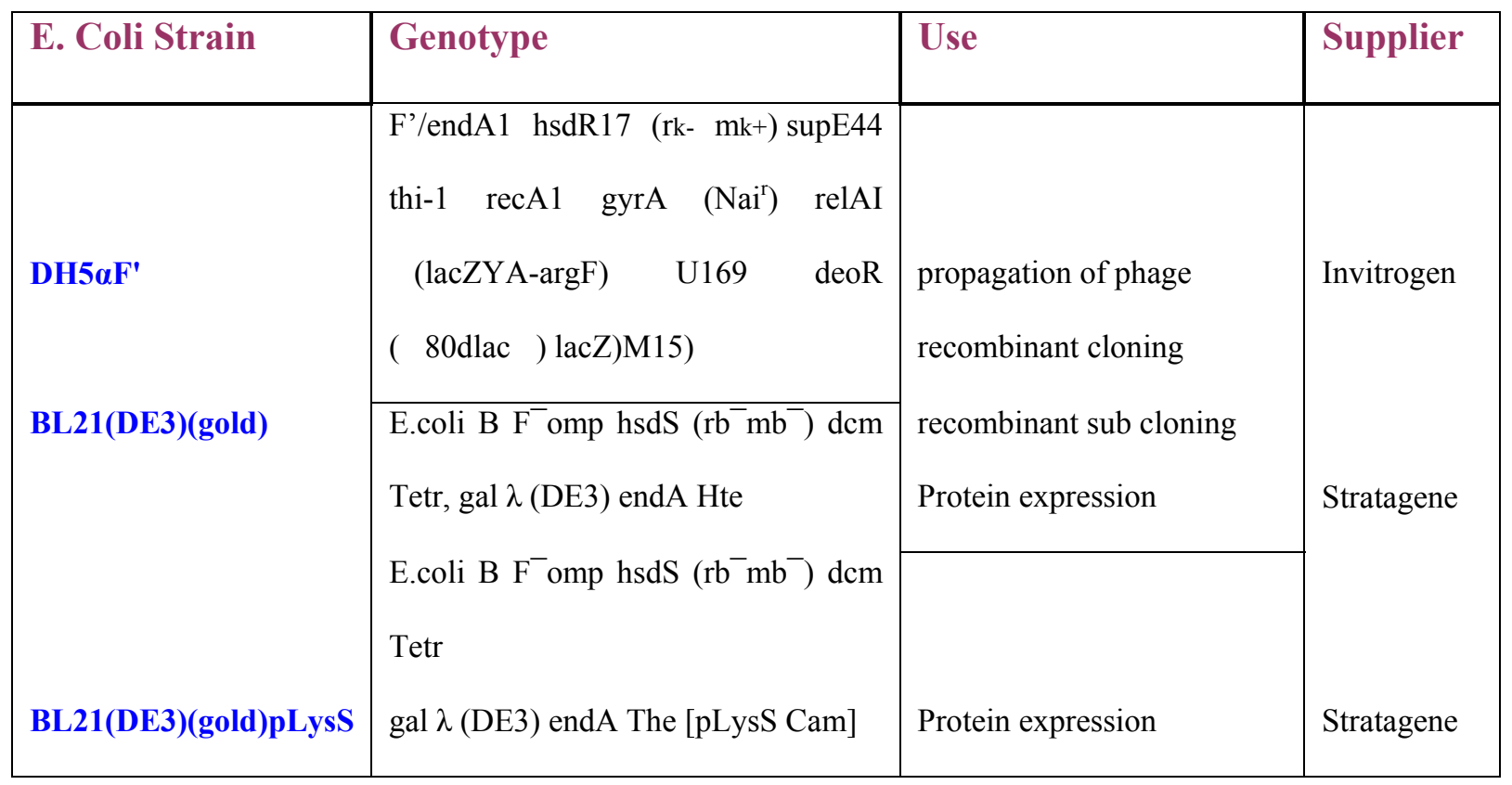

\subsubsection{Bacterial plasmids:}

\begin{tabular}{|l|l|l|}
\hline plasmid & Use & supplier \\
\hline pET-28b(+) & protein expression & Novagen \\
\hline Pmal-p2x & cloning MBP tag & New England Biolabs \\
\hline pET-42 & cloning GST tag & EMB biosciences \\
\hline pET-32 & cloning Thrx tag & EMB biosciences \\
\hline pTYB1 & cloning Chitin tag & New England Biolabs \\
\hline
\end{tabular}

\subsubsection{Primers:}

They were either supplied by Operon technologies or by MWG. 


\subsubsection{Primers used for tag amplification}

\begin{tabular}{|c|c|}
\hline Sfi-Tag & GCAGGGGCCGCCCTGGCC + bp's from beginning of tag \\
\hline SfiI-CBP & $\begin{array}{l}\text { GCAGGGGCCGCCCTGGCCGCAGAAAAGAGAAGATGG AAAAAGAAT } \\
\text { TTCATAGCCGTCTCAGCAGCCAACCGC TTT }\end{array}$ \\
\hline SfiI-MBP & GCAGGGGCCGCCCTGGCCGCCGCCAAAATCGAAGAAGGTAAAGCA \\
\hline SfiI-GST & GGGGCCGCCCTGGCCGCGTCCCCTATACTAGGTTATTGG \\
\hline SfiI-Thrx & $\begin{array}{l}\text { GCAGGGGCCGCCCTGGCCGCGAGCGATAAAATTATTCACCTGCTGG } \\
\text { TAATC }\end{array}$ \\
\hline SfiI-strep & $\begin{array}{l}\text { GCAGGGGCCGCCCTGGCCGCAGCTTGGAGCCACCCGCAGTTCGAA } \\
\text { AAA }\end{array}$ \\
\hline SfiI-chitin & GCAGGGGCCGCCCTGGCCGCGACGACAAATCCTGGTGTATCCGCT \\
\hline AgeI-Tag & $\begin{array}{l}\text { Last bps of the tags + GGAGGCGGTTCAGGCGGAGGTGGCTCTGGCG } \\
\text { GT+ ACCGGTCCGG }\end{array}$ \\
\hline $\begin{array}{l}\text { AgeI- } \\
\text { CBP(rc) }\end{array}$ & $\begin{array}{l}\text { CCGGACCGGTACCGCCAGAGCCACCTCCGCCTGAACCGCCTCCAA } \\
\text { GTGCCCCGGAGGATGAGATTTTCTTAAAGCGGTTGGCTGCTGAGACG } \\
\text { GC }\end{array}$ \\
\hline $\begin{array}{l}\text { AgeI- } \\
\text { MBP(rc) }\end{array}$ & $\begin{array}{l}\text { CCGGACCGGTACCGCCAGAGCCACCTCCGCCTGAACCGCCTCCAG } \\
\text { TCTGCGCGTCTTTCAGGGCTTC }\end{array}$ \\
\hline $\begin{array}{l}\text { AgeI- } \\
\text { GST(rc) }\end{array}$ & $\begin{array}{l}\text { CCGGACCGGTACCGCCAGAGCCACCTCCGCCTGAACCGCCTCCAT } \\
\text { CCGATTTTGGAGGATGGTCGCC }\end{array}$ \\
\hline $\begin{array}{l}\text { AgeI- } \\
\operatorname{Thrx}(\mathbf{r c})\end{array}$ & $\begin{array}{l}\text { CCGGACCGGTACCGCCAGAGCCACCTCCGCCTGAACCGCCTCCGG } \\
\text { CCAGGTTAGCGTCGAGGAACTC }\end{array}$ \\
\hline $\begin{array}{l}\text { AgeI- } \\
\text { strep(rc) }\end{array}$ & $\begin{array}{l}\text { CCGGACCGGTACCGCCAGAGCCACCTCCGCCTGAACCGCCTCCTT } \\
\text { TTTCGAACTGCGGGTGGCTCCA }\end{array}$ \\
\hline AgeI- & $\begin{array}{l}\text { CCGGACCGGTACCGCCAGAGCCACCTCCGCCTGAACCGCCTCCTT } \\
\text { GAAGCTGCCACAAGGCAGGAAC }\end{array}$ \\
\hline
\end{tabular}




\subsubsection{Other primers:}

\begin{tabular}{|c|l|}
\hline T7 promoter & TAATACGACTCACTATAGGG \\
\hline T7 terminator & GCTAGTTATTGCTCAGCGG \\
\hline pDpH5' & GCAGCCGCTGGATTGTTATTA \\
\hline pDpH3' & TTGTCGTCTTTCCAGACGTTA \\
\hline laclseq & CACCGGAAGGAGCTGACTGGGTTG \\
\hline lacmid5 & ATCCTGGTCATCCAGCGGATAGTT \\
\hline lacseq3 & AGCCCAGTAGTAGGTTGAGGCCGTT \\
\hline LacIEcorVlong & $\begin{array}{l}\text { TCGTATCCCACTACCGAGATATCCGCACCAACGCGCAG } \\
\text { CCCGGACTCGGTAATGGCA }\end{array}$ \\
\hline LacIMlul3' & TCTCGCGCAACGCGTCAGTGGGCTGATCATTAACT \\
\hline pelBSfil & $\begin{array}{l}\text { GGCATGCGCGCCACCGGTCTCATCTTTATAATCGGCAG } \\
\text { GGCGGCCCCTGCTGCGAGTAATAA }\end{array}$ \\
\hline pelBNcol & AAGCTTGCCAAATTCTATTTCAAGGAGACAGTCACCATGG \\
& AATACCTATTGCC \\
\hline Lacl & CGCATTGCGCCCAGCGCCATCTGATCGTTGGC \\
\hline LacImut & AGATGGCCATTACCGCGCTGGGCGCAATGCGT \\
\hline MBPmutg5 & GAAAGCGAAAGGTAAGAGCGCTCTGATGTTCAA \\
\hline MBPmutgnew3 & GCGCTCTTACCTTTCGCTTTCAGTTCTTTAT \\
\hline Fwdantifluor & GCTCATGCGCGCATGCCGTCGTTATGACTCAAACACCAC \\
\hline Revantifluor & CGATC \\
\hline MBP5 for sequencing & GATAACGCTAGAGGAGACGGTGACTGAGGTTCCTTG \\
\hline
\end{tabular}

\subsection{Polymerase Chain Reaction PCR:}

PCR was performed using BioRad 8-Tube Strip CLR pcr tubes. The final volume of the reaction was usually $20 \mu$, but scaled up in the case of need of larger amounts for further cloning. The pcr master mix was prepared on ice and the general recipe included $500 \mathrm{nM}$ of each primer, $250 \mu \mathrm{M}$ of each dNTP, 0.1 units of the polymerase (Vent (NEB, $2000 \mathrm{u} / \mathrm{ml}$ ), ex-Taq from TAKARA or NEB taq), proof reading polymerase was used in the cloning steps, $1 \mathrm{x}$ of pcr buffer and in the case magnesium was not into it, it was added to a final concentration of $2.5 \mathrm{mM}$. The target DNA was added in amounts up to $1 \mathrm{ng}$ of plasmid. In the case of using colonies to do pcr on their DNA, each one was picked with a sterile tip and transferred into a sterile pcr tube containing $50 \mu 1$ of 2 xty media. $3 \mu 1$ of this media was used as target DNA. The mix was adjusted in volume with sterilized 
double distilled water. All amplifications were performed using a MJ Research PCT-200 Peltier Thermal Cycler with the following profile: - initiation at $95^{\circ} \mathrm{C}$ for 4 min followed by $1 \mathrm{~min}$ at $92^{\circ} \mathrm{C}$ for denaturation, annealing at optimum temperature $\left(45-65^{\circ} \mathrm{C}\right)$ for 1 min and extension at $72^{\circ} \mathrm{C}$ for 1 min per kbp of final product. The cycle from denaturation to extension was then repeated 29 more times. At the end of the cycles, a final step at $72^{\circ} \mathrm{C}$ for 5 min was carried out and then it remained at $4^{\circ} \mathrm{C}$ until taken out of the machine.

\subsection{Antibiotic concentrations: (stocks 1000x concentrated)}

Carbenicillin: $50 \mu \mathrm{g} / \mu \mathrm{l}$

Kanamycin: $50 \mu \mathrm{g} / \mu 1$

Tetracycline: $15 \mathrm{mg} / \mathrm{ml}$

\subsection{Restriction Enzyme Digestion of DNA}

DNA was digested with restriction enzymes according to the manufacturer's instructions.

\subsection{Ligation of DNA Fragments}

DNA fragments were ligated in a $10 \mu 1$ reaction mix containing; 3 units of T4 DNA ligase (Promega), 1x T4 ligation buffer, cut vector/insert DNA (concentration between 100-250 ng) in a molar ratio of 1:3 and de-ionized water to make up the final volume. Ligation samples were incubated at $16^{\circ} \mathrm{C}$ for $16-20 \mathrm{~h}$ and stored at $4{ }^{\circ} \mathrm{C}$ prior to use. The ligation mix was added directly to competent $E$. coli cells for transformation 


\subsection{DNA Agarose Gel Electrophoresis}

This method was used to quantify and characterise DNA that had been extracted, purified, digested with restriction enzymes and amplified via PCR. Agarose (electrophoresis grade; Gibco BRL ${ }^{\circledR}$ Life Technologies) gels between 0.7-1.2\% (w/v) were made with 1XTAE buffer containing $0.5 \mu \mathrm{g} / \mathrm{ml}$ of ethidium bromide (EtBr). DNA samples were mixed with $10 \mathrm{x}$ DNA loading dye (recipe follows) and loaded into the agarose gel submerged in $1 \times$ TAE buffer. A voltage of $1-5 \mathrm{~V} / \mathrm{cm}$ was applied until the DNA had migrated to the desired position. DNA products were sized against Lambda HindIII (Promega) and phiX Hae III DNA ladder (Promega) markers. DNA Products were visualised with Syngene BioImaging with GeneSnap software from SynGene.

50x TAE (Tris-acetate) (1L)

- Dissolve $242 \mathrm{~g}$ Tris in $500 \mathrm{ml} \mathrm{H}_{2} \mathrm{O}$

- Add $100 \mathrm{ml}$ 0.5 $\mathrm{M} \mathrm{Na}_{2}$ EDTA (pH 8.0) and $57.1 \mathrm{ml}$ glacial acetic acid

- Adjust volume to 1liter with $\mathrm{H}_{2} \mathrm{O}$

- Store at room temperature

10x DNA loading Dye: Final concentrations:

- $50 \%$ glycerol

- $\quad 0.1 \%$ Bromophenol blue (BB)

- $\quad 0.1 \%$ Xylene Cyanole FF (XC)

For $10 \mathrm{ml}:$

- $1 \mathrm{ml} \mathrm{1 \%} \mathrm{BB} 1 \mathrm{ml} \mathrm{1 \%}$

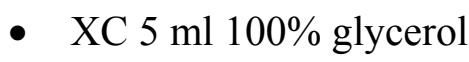

- $3 \mathrm{ml} 2 \mathrm{xdH} 2 \mathrm{O}$ 


\subsection{DNA recovery from agarose gel}

DNA was recovered using the QIAquick ${ }^{\mathrm{TM}}$ Gel Extraction Kit (Qiagen) according to the manufacturer's instructions.

\subsection{Plasmid DNA preparation from Bacteria}

Plasmid DNA (up to $20 \mu \mathrm{g}$ ) was purified from E. coli small-scale culture using the QIAprep $^{\circledR}$ Spin Miniprep Kit (Qiagen). DNA was recovered according to the manufacturer's instructions.

\subsection{Transformation of DNA (ligation or plasmid) into competent cells}

In-house Cells normally used:

- Chemically competent $(\mathrm{DH} 5 \mathrm{aF}-\mathrm{T})=2 \mathrm{X} 10 \mathrm{e} 8$

- Electrocompetent (BL21 DE3) 2.5 X10e10

\subsubsection{Electrocompetent:}

Set the electroporator, BTX Harvard Apparatus Electro Cell Manipulator ECM630

Bio Rad Gene Pulser II and BioRad Pulse Controller II, with the following settings:

- Voltage: 2500V HV

- Resitor: $200 \Omega$

- Capacitor: $25 \mu \mathrm{F}$

Place electroporation cuvettes (BTX Electroporation Cuvettes Plus 2mm Gap) on ice. Place $50 \mu 1$ of electro-competent competent cells inside of the cuvette. Add carefully DNA to the cells (1-10 ng plasmid DNA). Electroporate and add $500 \mu$ lof S.O.C. Incubate for one hour at $260 \mathrm{rpm}$ and $37^{\circ} \mathrm{C}$. 


\subsubsection{S.O.C. Recipe :}

Difco SOB medium+20mM Glucose

Sterilize by autoclaving

\subsubsection{Chemical competent}

-Place $20 \mu \mathrm{l}$ of chemically competent cells into tube ( Fisherbrand Culture Test Tube $12 X 75 \mathrm{~mm}$ polystyrene with cap) while on ice. Add DNA and incubate in water bath at $42^{\circ} \mathrm{C}$ for 30 s. Incubate in ice for two minutes. Add $200 \mu 1$ of S.O.C. Incubate for one hour at $260 \mathrm{rpm}$ and $37^{\circ} \mathrm{C}$.

\subsection{Selection of antibodies using streptavidin magnetic beads:}

The selection process was done using KingFisher ${ }^{\circledR}$ (Thermo-Labsystems)

\section{Consumables/Reagents Required for selection}

- 1xKingfisher 96 well plate (thermo-Labsystems, \#97002080, 100 pieces)

- 1x KingFisher Tips Combs (thermo-Labsystems, \#97002070, 50 pieces)

- Dynabeads (Streptavidin M-280 magnetic beads, $6.7 \times 10^{\wedge} 8$ beads $/ \mathrm{ml}, \# 112.06$ )

-Wash buffer 1xPBS-T (0.1\% tween 20). 100 ul of 50\% Tween in PBS in $50 \mathrm{ml} 1 x$ PBS.

Filter

-Wash buffer 1x PBS-LT (0.01\% tween20). 10 ul of 50\%Tween in PBS in $50 \mathrm{ml} 1 \mathrm{xPBS}$.

Filter

-Elution buffer: $0.1 \mathrm{M} \mathrm{HCl}$

-Neutralizing buffer: $1.5 \mathrm{M}$ Tris $\mathrm{pH}=8.8$

Beads and library preparation prior to selection:

Beads: 
- 30ul of beads are required for each sample tested

- wash twice with PBS-T

- resuspend each set in $200 \mu 1$ of PBS-T

Blocking of the library

- $\quad 2 \% \mathrm{BSA} .1 \mathrm{~g}$ in $50 \mathrm{ml} \mathrm{H} 2 \mathrm{O}$.

- Block the library using 2\% BSA (stock conc.) in PBS-LT.

- per sample $100 \mathrm{ul} \mathrm{library+37ul} \mathrm{PBS-LT+34ul} \mathrm{2 \% BSA.}$

- which will mean $1.010^{\wedge} 2 \mathrm{cfu}$ in $100 \mathrm{ul}$ and $0.4 \% \mathrm{BSA}$ final.

Block rotating at RT for $1 \mathrm{hr}$.

\section{KingFisher plate:}

The plate was set up for selection as shown in the diagram

\begin{tabular}{|c|c|c|c|c|c|c}
\cline { 2 - 6 } \multicolumn{1}{c|}{} & prot\# 1 & prot\# 2 & prot\# 3 & prot\# 4 & prot\# 5 & vol \\
\hline A & $\begin{array}{c}\text { antigen } \\
+\phi\end{array}$ & $\begin{array}{c}\text { antigen } \\
+\phi\end{array}$ & $\begin{array}{c}\text { antigen } \\
+\phi\end{array}$ & $\begin{array}{c}\text { antigen } \\
+\phi\end{array}$ & $\begin{array}{c}\text { antigen } \\
+\phi\end{array}$ & $\begin{array}{c}171 \mu l \\
\text { lib }+19 \mu l \text { antigen } \\
\text { incubate 1hr RT }\end{array}$ \\
\hline B & empty beads & empty beads & empty beads & empty beads & empty beads & $200 \mu \mathrm{l}$ \\
\hline C & PBS-T & PBS-T & PBS-T & PBS-T & PBS-T & $190 \mu l$ \\
\hline D & PBS-T & PBS-T & PBS-T & PBS-T & PBS-T & $190 \mu \mathrm{l}$ \\
\hline E & PBS-LT & PBS-LT & PBS-LT & PBS-LT & PBS-LT & $190 \mu \mathrm{l}$ \\
\hline F & PBS-LT & PBS-LT & PBS-LT & PBS-LT & PBS-LT & $190 \mu \mathrm{l}$ \\
\hline G & PBS-LT & PBS-LT & PBS-LT & PBS-LT & PBS-LT & $190 \mu \mathrm{l}$ \\
\hline H & $0.1 \mathrm{M} \mathrm{HCl}$ & $0.1 \mathrm{M} \mathrm{HCl}$ & $0.1 \mathrm{M} \mathrm{HCl}$ & $0.1 \mathrm{M} \mathrm{HCl}$ & $0.1 \mathrm{M} \mathrm{HCl}$ & $150 \mu \mathrm{l}$ \\
\hline
\end{tabular}

Each round had two parts;

1) Interaction and elution of phages with the antigen

2) Amplification of the output

Library used was a tertiary phage library with a concentration of $1.0 \times 10^{10} \mathrm{cfu} / \mu \mathrm{l}$.

1) Interaction and elution of phages with the antigen

The whole process takes place in KingFisher plates 
- Incubation of the blocked library and antigens for an hour at room temperature

- Incubation of the mixture of library and antigen with the streptavidin magnetic beads (Dynabeads, DYNAL) for $15 \mathrm{~min}$ at room temperature

- Elution with $\mathrm{HCl}$ for $3.5 \mathrm{~min}$ followed by immediate neutralization with $50 \mu 1$ of $1.5 \mathrm{M}$ tris $\mathrm{pH}=8.8$.

- $100 \mu \mathrm{l}$ of the neutralized eluted phages, was used to infect $1 \mathrm{ml}$ of DH5 F cells at $\mathrm{OD}_{600}=0.5$ and left to incubate at $37^{\circ} \mathrm{C}$ for $45 \mathrm{~min}$ in steady state.

- $5 \mu 1$ of the infected bacteria was plated on Ampicillin $(0.1 \mathrm{mg} / \mathrm{ml}$ final $) /$ glucose $(3 \%$ final) agar plates to give us an idea of the order of magnitude of the output.

- The remaining output was plated on a large Ampicillin/glucose agar plate Both plates were incubated over night at $30^{\circ} \mathrm{C}$.

2) Amplification of the output

- Scrape off the cells from the large Ampicillin plate in $2 \mathrm{ml}$ of 2xTy media

- Use $10 \mu 1$ of the resuspended cells to inoculate $10 \mathrm{ml}$ of $2 \mathrm{xTy}(\mathrm{Amp} / \mathrm{Glu})$ media and incubated at $37^{\circ} \mathrm{C}$ to reach a density of $\mathrm{OD}_{600}=0.5$.

- Add $1.3 \mathrm{ml}$ of helper phage to the bacteria and incubate standing for $45 \mathrm{~min}$

- Centrifuge for $10 \mathrm{~min}$ at $3,700 \mathrm{rpm}$

- Resuspend cell pellet in $10 \mathrm{ml}$ of 2xTy, Ampicillin, Kanamycin media

- Incubate over night at $30^{\circ} \mathrm{C}, 260 \mathrm{rpm}$.

- Centrifuge at 3,000rpm for $20 \mathrm{~min}$ and recover the supernatant containing phages

- Remove a $500 \mu 1$ aliquot and use as input for the next selection round.

- PEG precipitate remaining phages (chapter 2: PEG precipitation of phages) 
Each selection was comprised of three rounds where the antigen concentration and the washes were varied as shown in the table

\begin{tabular}{|c|c|c|c|}
\cline { 2 - 4 } \multicolumn{1}{c|}{} & 1st round & 2nd round & 3rd round \\
\hline antigen concentration & $0.6 \mu \mathrm{M}$ & $0.06 \mu \mathrm{M}$ & $6 \mathrm{nM}$ \\
\hline washes & $1 \mathrm{~min}$ & $5 \mathrm{~min}$ & $15 \mathrm{~min}$ \\
\hline
\end{tabular}

\subsection{Immunotube selections:}

\section{Day 1:}

To a 75x12 mm NUC immunotube (Maxisorp) add 10 $\mu \mathrm{g}$ of antigen in 4ml of PBS.

Leave overnight to coat.

Day 2:

Start a $10 \mathrm{ml}$ of DH5 $\alpha$ FT culture in $2 \mathrm{xTy} / 3 \%$ glucose $/ 1 \mathrm{x}$ tetracycline $(15 \mu \mathrm{g} / \mathrm{ml})(\mathrm{per}$ immunotube). $37^{\circ} \mathrm{C}$, shaking

Wash tube 2-3 times with PBS (simply pour in and pour out again immediately)

Block by filling the tube to the brim with $2 \%$ milk in PBS (MPBS). Cover with parafilm.

$37^{\circ} \mathrm{C}$, rotating, $1-2 \mathrm{hrs}$

Pre-blocking the library: Make up $10^{13}$ tu library phage to $4 \mathrm{ml}$ of $2 \%$ MPBS $(2 \mathrm{ml}$ of $4 \%$

MPBS, 1ml PBS, 1ml library phage). Room temperature (RT), 30min, rotating.

Wash immunotube 3 times with PBS (3xPBS)

Add the blocked library to blocked immunotube. $30 \mathrm{~min}$, RT, rotating and then $1.5 \mathrm{hrs}$,

RT, standing.

Wash tube:

Round $1 \rightarrow 2 x$ PBST (PBS with $0.1 \%$ Tween 20) then, 2x PBS.

Round $2 \rightarrow$ 10x PBST, then, 10x PBS. 
Round $3 \rightarrow$ 20x PBST, then, 20x PBS.

Elute phage with $1 \mathrm{~m}$ of $1 \mathrm{mg} / \mathrm{ml}$ trypsin freshly diluted in PBS. (add $900 \mu 1$ of PBS to the $100 \mu 1$ of trypsin $(10 \mathrm{mg} / \mathrm{ml}) .30 \mathrm{~min}, 37^{\circ} \mathrm{C}$, standing.

Add $10 \mu \mathrm{l}$ of trypsin eluate $\mathrm{t} 10 \mathrm{ml}$ of DH5 $\alpha \mathrm{FT}$ at O.D. $0.5 .30 \mathrm{~min}, 37^{\circ} \mathrm{C}$, standing.

Titrate $10 \mu 1$ on Amp/Tet/Glu plate:

For $2^{\text {nd }}$ and $3^{\text {rd }}$ round:

- Dilute $10 \mu 1$ into $90 \mu 1$ of media and plate on Amp/Tet/Glu plate

- Dilute $1 \mu 1$ into $90 \mu 1$ of media and plate on Amp/Tet/Glu plate

To be able to pick single colonies for ELISA.

Spin down the cells. 10min, 3700rpm.

Decant supernatant and resuspend the pellet on the little liquid left $(\sim 100 \mu 1)$.

Plate the cells on Amp/Tet/Glu. $37^{\circ} \mathrm{C}$, overnight.

Day 3:

Scrape the lawn in approximately $2 \mathrm{ml}$ media with $15 \%$ glycerol.

Use $10 \mu \mathrm{l}$ to grow in $10 \mathrm{ml}$ of $2 \mathrm{xTy}$ Amp/Tet/Glu, till O.D. $0.5 .37^{\circ} \mathrm{C}$, shaking.

Add $2 \times 10^{10}$ of warm helper phage (at $37^{\circ} \mathrm{C}$, $)\left(200 \mu 1\right.$ of $1 \times 10^{11}$ stock) $(20: 1) .37^{\circ} \mathrm{C}$,

30min, standing.

Streak on Amp/Glu, Kan/Glu, plates to check for helper phage infection (1 $\mu 1)$. (library

plasmid has Amp resistance and Helper phage has Kan).

Spin down. 10min, 3000rpm.

Resuspend on Amp/Kan media (50ml). $30^{\circ} \mathrm{C}$, overnight).

Coat a new immunotube for next selection round.

Day 4: 
Double PEG precipitate

Use $100 \mu 1$ for the next selection round.

\subsection{PEG precipitation of phages}

- Add $2 \mathrm{ml}$ of pre-chilled $20 \% \mathrm{PEG}+2.5 \mathrm{M} \mathrm{NaCl}$ to each $10 \mathrm{ml}$ supernatant sample

- Incubate on ice for $1 \mathrm{hr}$ to aid precipitation

- Centrifuge the phage precipitate at 3,000rpm for $30 \mathrm{~min}$ and discard supernatant

- Re-suspend each pellet in 1 $\mathrm{ml}$ PBS

- Centrifuge the phage sample at 13,000 rpm for $15 \mathrm{~min}$ (to pellet bacterial pellet debris)

- Recover the $1 \mathrm{ml}$ supernatant into a fresh microcentrifuge tube containing 167ul $\mathrm{PEG}+\mathrm{NaCl}$

- Mix and incubate on ice to aid precipitation

- Centrifuge at 13,000 rpm for 10min (to pellet precipitated phage) and discard supernatant

Briefly re-centrifuge (for $1 \mathrm{~min}$ ) to recover traces of PEG

Re-suspend the phage pellet in 200ul of PBS and centrifuge at 13,000 rpm for 5 min (to pellet residual cell debris)

Transfer the phage containing supernatant to a fresh microcentrifuge tube and store at $4^{\circ} \mathrm{C}$

\subsection{Protein expression:}

\subsubsection{Expression of proteins using IPTG as inductor}

This protocol can be used to express recombinant proteins from plasmids with lac operon (e.g. pDan5 and pRosa plasmids). The cell strains used in each case was different as the pRosa vectors, being a derivative from $\mathrm{pET} 28^{1}$ vector, needed the presence of the T7 polymerase in order to start expression. The presence of this polymerase is indicated in the genotype by DE3 and the strain 
used in this cases was either BL21(DE3)(gold) or BL21-pLYsS(DE3). In the case of pDAN5 vector the strains used were $\mathrm{DH} 5 \alpha \mathrm{F}$ ' or DH5 $\alpha \mathrm{F}^{\prime} \mathrm{T}$. When using BL21-pLYsS(DE3) growth temperature can be higher, $37^{\circ} \mathrm{C}$, than when using $\mathrm{BL} 21$ (DE3)(gold) as they are more tightly regulated, the possibility of leak is lower and thus toxicity is lower.

Induction of scFvs from pRosa vectors in BL21-pLysS or BL21(gold) with 2xTy media:

Volume of media can vary. The flask should vary accordingly so it is always 5 times larger than the volume of media.

- The growth media will be $2 x$ Ty with $3 \%$ of glucose and $1 x$ of Kan

- inoculate the media using several colonies from over night plate grown at $37^{\circ} \mathrm{C}$

- incubate at $30^{\circ} \mathrm{C}$ shaking at $260 \mathrm{rpm}$ until OD $\sim 0.8$

- prepare induction media: same volume as earlier of 2xTy, 1x Kan and $250 \mu \mathrm{M} \mathrm{IPTG}$

- Spin down and resuspend the pellet in the induction media

- Incubate over night at $25^{\circ} \mathrm{C}$ and $260 \mathrm{rpm}$

- Spin down and keep the pellet in the $-80^{\circ} \mathrm{C}$ freezer of proceed to extraction of the protein.

\subsubsection{Expression of proteins using autoinducing media}

As explained in paper:

Studier, F. W., Protein production by auto-induction in high density shaking cultures.

Protein Expr Purif 2005, 41, (1), 207-34.

\subsection{Periplasmic extraction protocol}

\section{Buffers:}

$-5 \mathrm{mM} \mathrm{MgSO} 4$

- PPB Buffer 


\subsubsection{1-PPB buffer (for a final vol of 1L):}

$-50 \mathrm{mM}$ tris- $\mathrm{HCl}(\mathrm{pH}=7.6)$

$-25 \mathrm{ml}$ of $2 \mathrm{M}$ tris $\mathrm{pH}=7.5$

-1 mM EDTA

- $2 \mathrm{ml}$ of $0.5 \mathrm{M}$ EDTA

$20 \%$ Saccharose (sucrose)

$-20 \mathrm{~g}$ of dry sucrose

Filter sterilize with $0.2 \mu \mathrm{m}$ filter.

Note: This protocol can be scaled up or down depending on the amount of bacteria working with. Some variations on the volume can be done in order to get more or less concentrated extract.

1) Starting with a $50 \mathrm{ml}$ culture of induced bacteria. Centrifuge culture at $4000 \mathrm{rpm}, 10$ min.at $4^{\circ} \mathrm{C}$.

3) Re-suspend pellet in $1.25 \mathrm{ml}$ cold PPB buffer, transfer into micro centrifuge tubes and incubate on ice for $20 \mathrm{~min}$.

4) Spin at $14000 \mathrm{rpm}$ for $7 \mathrm{~min}$ in cold and transfer the supernatant to a sterile tube.

5) Resuspend the bacterial pellet in $500 \mu 1$ of $5 \mathrm{mM} \mathrm{MgSO} 4$ (or $\mathrm{MgCl}$ ). Incubate at $\mathrm{RT}$ for $10 \mathrm{~min}$. Spin at 10,000 rpm for $7 \mathrm{~min}$. Combine the supernatants.

6) Spin combined supernatant at $14000 \mathrm{rpm}$ for $7 \mathrm{~min}$ to remove small particles, and transfer to a new tube.

Final volume $1750 \mu 1$ of p.e. 


\subsection{LSRII :}

\subsection{1 parameters:}

\begin{tabular}{|c|c|c|c|c|c|}
\hline \multirow{2}{*}{ Loader Settings } & & \multicolumn{2}{|c|}{ Parameters } & \multirow[b]{2}{*}{$\mathbf{A}$} & \multirow[b]{2}{*}{$\mathrm{H}$} \\
\hline & Filters & Voltage & Log & & \\
\hline Flow rate $=0.5 \mathrm{ul} / \mathrm{sec}$ & $\mathrm{FSC}$ & 313 & $\mathrm{Y}$ & $\bar{Y}$ & - \\
\hline Sample volume $=25 \mathrm{ul}$ & SSC & 177 & $\bar{Y}$ & $\mathrm{Y}$ & - \\
\hline Mixing Volume $=100 u l$ & FITC & 650 & $\bar{Y}$ & $\mathrm{Y}$ & $\bar{Y}$ \\
\hline Mixing Speed $=250 \mathrm{ul} / \mathrm{min}$ & APC-Cy7 & 381 & $\bar{Y}$ & $\mathrm{Y}$ & $\bar{Y}$ \\
\hline Number of mixes $=5$ & APC-Cy 7 & 266 & $\mathrm{Y}$ & $\mathrm{Y}$ & $\bar{Y}$ \\
\hline Wash volume 800ul & & & & & \\
\hline
\end{tabular}

\subsection{SV5 ELISA}

\section{Consumables:}

- Mouse immunoglobulins/AP (D0486, Dakocytomation)

- SV5 antibody

- $\quad$ NUNC Maxisorp ${ }^{\mathrm{TM}}$ plates (96 and 384 wells) (eBiosciences)

- $\quad$ PBS (Fisher)

- Tween-20 (SIGMA)

- Neutravidin (Pierce)

- Wonder block: skim milk, fish gelatin and BSA, all of them at $0.3 \%$ dissolved in 1x PBS.

- NBT/BCIP, Pierce 
Volumes and concentrations:

\begin{tabular}{|c|c|c|}
\cline { 2 - 3 } \multicolumn{1}{c|}{} & \multicolumn{2}{c|}{ volume in plates } \\
\cline { 2 - 3 } \multicolumn{1}{c|}{} & $\begin{array}{c}384 \text { well } \\
(\mu \mathrm{l})\end{array}$ & $\begin{array}{c}\mathbf{9 6} \text { well } \\
(\mu \mathrm{l})\end{array}$ \\
\hline antigen & 50 & 100 \\
\hline washings & 100 & 200 \\
\hline blocking agent & 100 & 200 \\
\hline antibodies & 50 & 100 \\
\hline app-substrate & 80 & 160 \\
\hline
\end{tabular}

\begin{tabular}{|c|c|c|}
\cline { 3 - 3 } \multicolumn{1}{c|}{} & final & \multicolumn{1}{c|}{} \\
\cline { 3 - 3 } \multicolumn{1}{c|}{} & concentration & amount/well \\
\hline neutravidin & & $0.5-1 \mu \mathrm{g}$ \\
\hline antigen & & $0.1-1 \mu \mathrm{g}$ \\
\hline$\alpha$-SV5 & $5 \mu \mathrm{g} / \mathrm{ml}$ & $50-100 \mu \mathrm{l}$ \\
\hline$\alpha$-mouse & $5 \mu \mathrm{g} / \mathrm{ml}$ & $50-100 \mu \mathrm{l}$ \\
\hline
\end{tabular}

All the steps are done at room temperature with no shaking unless indicated.

1- Coat over night with neutravidin at $4^{\circ} \mathrm{C}$. If coating the plate directly with the antigen, start in step \# 1.1 and skip steps \#4 \& 5

1.1- Coat with antigen over night at $4^{\circ} \mathrm{C}$ or $1 \mathrm{hr}$ at $37^{\circ} \mathrm{C}$ (skip this step for neutravidin coated plates)

2- Wash twice with PBS-LT (1xPBS with $0.01 \%$ tween-20)

3- Block with wonder block for $1 \mathrm{hr}$

4- Incubate plates with biotinylated antigen for 1 hour at $37^{\circ} \mathrm{C}$ (skip this step for no neutravidin coated plates).

5- Wash two times with PBS-LT

6- Add expressed scFvs and incubate for $1.5 \mathrm{hrs}$.

7- Wash two times with PBS-T and two more times with PBS-LT

8- Add anti-SV5 antibody and incubate for $1 \mathrm{hr}$

9- Wash three times with PBS-T and three more with PBS-LT

10- Add anti-mouse-ap and incubate for $1 \mathrm{hr}$

11- Wash three times with PBS-T and three more with PBS-LT

12- Develop the assay with alkano phosphatase susbstrate was used NBT/BCIP

13- Read absorbance at $405 \mathrm{~nm}$ using the SPECTRAfluor Plus spectrometer. 
Some modifications that sometimes were done to this procedure were:

- Use of $\alpha$-mouse-HRP as secondary antibody. The way to develop the assay is by the use of $100 \mu 1$ of TMB-ELISA substrate (Pierce); the signal was quenched with $50 \mu \mathrm{l}$ of $1 \mathrm{M} \mathrm{H}_{2} \mathrm{SO}_{4}$. Readings were performed at $450 \mathrm{~nm}$.

\subsection{SDS polyacrylamide gel electrophoresis}

\section{Supplies:}

- NuPAGETM 4-12\% Bis-Tris gels, Invitrogen, with either 12 or 17 wells.

- The gel apparatus was Novex Mini-cell, Invitrogen.

- Power supply BioRad PowerPac HC or BioRad MODEL 200/2.0 Power Supply.

- 1x MES running buffer (20x buffer having the following composition 1M MES, $1 \mathrm{M}$ tris Base, 69.3mM SDS, 20.5mM EDTA (acid free).

- $4 \mathrm{x}$ denaturing loading dye (recipe at the en of the protocol)

\section{Procedure:}

Samples with $4 \mathrm{x}$ denaturing loading dye were boiled at $100^{\circ} \mathrm{C}$ for $7 \mathrm{~min}$, volume of sample plus loading dye was usually around $20 \mu$. The gel was pre-rinsed with distilled water and placed in the gel apparatus, buffer compartments were then filled and comb removed to uncover the wells. The samples were then loaded in gel. $7 \mu$ l of protein marker used was (Precision-plus Kaleidoscope (BIORAD.)) Power supply was set to apply 200V for $35 \mathrm{~min}$. The gel was then taken out of the plastic case and rinsed with double distilled water and developed by staining with gel code of silver. (Recipe follows)

Note: In case of the purification using affinity tags (Chapter 3, section 3.5). it was loaded on the gel $10 \mu 1$ of eluted fraction with $5 \mu 1$ of loading dye. 


\subsubsection{0x protein loading dye $(10 \mathrm{ml})$ :}

$2 \mathrm{ml} 1 \mathrm{M}$ Tris-HCL; pH 6.8

$0.8 \mathrm{~g}$ SDS

$4 \mathrm{ml} \mathrm{10 \%} \mathrm{glycerol}$

$0.4 \mathrm{ml} 14.7 \mathrm{~b}$-mercaptoethanol

$1 \mathrm{ml} 0.5 \mathrm{M}$ EDTA

$8 \mathrm{mg}$ Bromophenol Blue

\subsubsection{Gel Code staining}

\section{Consumables and buffers:}

- Fix Buffer: 50\% Methanol, 10\% Acetic acid

- Gel-Code stain (Pierce, cat\# 24590)

To a polyacrilamide protein gel.

- Rinse with nano-pure water; three times for $5 \mathrm{~min}$ each.

- Incubate the gel in Fix Buffer for 10 min.

- Re-hydrated gel in nano-pure water, three times for $5 \mathrm{~min}$ each. Not need to shake.

- Add Gel-code solution to cover the gel. Incubate for up to an hour.

- Destain gel with large volume of nano pure water.

All the steps were done at room temperature with slow rotation. Stained gels were then scanned. 


\subsubsection{Silver staining}

To a polyacrilamide protein gel.

\begin{tabular}{|c|c|c|c|c|}
\hline Step & Solutions & Recipes & Operation & Time \\
\hline 1 & Sol A & $50 \% \mathrm{MeOH} / 7 \% \mathrm{AcOH} / 43 \% \mathrm{H} 2 \mathrm{O}$ & Fix & $\begin{array}{l}2 \times 15 \\
\min \end{array}$ \\
\hline 2 & Sol B & $50 \% \mathrm{MeOH} / 50 \% \mathrm{H} 2 \mathrm{O}$ & Incubate & $10 \mathrm{~min}$ \\
\hline 3 & & $\mathrm{ddH} 2 \mathrm{O}$ & wash $2 x$ & $\begin{array}{l}2 \times 5 \\
\min \end{array}$ \\
\hline 4 & Sol C (fresh) & $0.02 \%$ Sodium thiosulfate $(\mathrm{Na} 2 \mathrm{~S} 2 \mathrm{O} 3)$ & Sensitize & $3 \min$ \\
\hline 5 & & $\mathrm{ddH} 2 \mathrm{O}$ & wash $2 x$ & $2 \times 1 \mathrm{~min}$ \\
\hline 6 & Sol D & $1.2 \%$ of Silver Nitrate $(\mathrm{AgNO} 3) 1 \mathrm{~N}$ & Incubate & $25 \mathrm{~min}$ \\
\hline 7 & & $\mathrm{ddH} 2 \mathrm{O}$ & wash $2 \mathrm{x}$ & $2 \times 1 \mathrm{~min}$ \\
\hline 8 & Sol E (fresh) & $\begin{array}{l}3 \% \text { Sodium Carbonate }(\mathrm{NaCO} 3) \\
0.05 \% \text { of } 37 \% \mathrm{HCOH} \text { (formaldehyde) } \\
2 \% \text { Sol C } \\
\mathrm{H} 2 \mathrm{O}\end{array}$ & Develop & $\sim 10 \mathrm{~min}$ \\
\hline 9 & Sol F & $5 \% \mathrm{AcOH} / 95 \% \mathrm{H} 2 \mathrm{O}$ & Stop & $10 \mathrm{~min}$ \\
\hline 10 & Sol G & $1 \% \mathrm{AcOH} / 99 \% \mathrm{H} 2 \mathrm{O}$ & Store & \\
\hline
\end{tabular}

\subsubsection{Gel transference into membrane (blotting):}

The SDS protein gel with the samples resolved on it was incubated for $10 \mathrm{~min}$ in $1 \mathrm{x}$ transfer buffer (Invitrogen). The gel was transferred to $0.2 \mu \mathrm{m}$ nitrocellulose membrane (Protran BA83; Schleicher \& Schuell Bioscience). The gel was placed over the membrane and in between 6 layers 
of filter paper (BioRad) in the XCell II ${ }^{\mathrm{TM}}$ Blot Module (Invitrogen). The current was set depending of the area of transference calculating the $\mathrm{mA}$ needed as $0.8 \mathrm{x} \mathrm{\textrm {cm } ^ { 2 }}$. The gel transfer was run for 50 $\min$.

\subsubsection{SV5 western}

Proteins blotted in a membrane.

All the steps are done at room temperature shaking. The development is done with no shaking.

The solutions are prepared in 1\% fish gelatin (F.G.) in 1x PBS

- $\quad$ xx anti-SV5 (1:1000)

- 1x anti-Mouse IgG-Alkaline Phosphatase (AP) (1:2000) (Dakocytomation)

Block the membrane for an hour with $4.5 \%$ fish gelatin in $1 \mathrm{xPBS}$. Incubate with $1 \mathrm{x}$ antiSV5 for $1 \mathrm{hr}$. Wash for 10 min with PBS-T and for 10 more min with PBS. Incubate for 1 hr with $1 \mathrm{x}$ anti-Mouse-ap. Wash for $15 \mathrm{~min}$ with PBS-T and for 15 min with PBS. Develop with 1 step NBT/BCIP substrate (Pierce).

\subsection{Beads for affinity tags:}

\begin{tabular}{|c|c|c|}
\cline { 2 - 3 } \multicolumn{1}{c|}{} & matrix & from \\
\hline MBP & amylose resin & New England Biolabs \\
\hline CBD & Chitin beads & New England Biolabs \\
\hline Strep-tag II & Strep-Tactin $\circledast$ Sepharose & IBA \\
\hline
\end{tabular}

\subsection{Tag purification; buffers and protocols:}

Consumables:

- Ni-NTA beads (BioRad)

- Chitin beads (NEB) 
- $\quad$ Strep- tactin agarose beads (IBA)

- $\quad$ Amylose beads(NEB)

- Micro Bio-spin Chromatography columns (Bio-Rad)

- Chemicals from SIGMA and Fisher

Each set of beads was pre-washed twice with the corresponding washing buffer, spinning at 5000rpm for 1 minute between washes.

\subsubsection{Purification using Ni-NTA beads:}

Add to the samples the same salts as in the wash buffer and in the same concentrations

\subsubsection{Wash buffer:} 1x PBS

$0.5 \mathrm{M} \mathrm{NaCl}$

$5 \mathrm{mM}$ imidazole

\section{Elution buffer:}

1x PBS

$0.5 \mathrm{M} \mathrm{NaCl}$

0.5 M imidazole

\subsection{Purification protocol:}

- wash beads 2 times with $1 \mathrm{ml}$ of wash buffer 1

- incubate sample and beads for 1 hour rotating at $4{ }^{\circ} \mathrm{C}$

- place beads in column and let sample flow by gravity

- wash with $3 \mathrm{ml}$ of wash buffer

- elute with three fractions of $100 \mu 1$ of elution buffer.

\subsubsection{Purification with Strep-Tactin Beads:}

\subsubsection{Washing buffers:}

Wash buffer 1: PBS-T (1x PBS with 0.1\% tween-20) 
Wash buffer 2: PBS-LT (1x PBS with $0.01 \%$ tween-20)

Wash buffer 3: 1x PBS

\subsubsection{Elution Buffer:}

$2.5 \mathrm{mM}$ desthiobiotin in 1xPBS. Use warm PBS to be able to dissolve the desthiobiotin.

\subsection{Purification protocol:}

- wash beads 2 times with $1 \mathrm{ml}$ of wash buffer 1

- incubate sample and beads for 1 hour rotating at $4{ }^{\circ} \mathrm{C}$

- place beads in column and let sample flow by gravity

- wash with $3 \mathrm{ml}$ of wash buffer 1

- wash with $3 \mathrm{ml}$ of wash buffer 2

- wash with $1 \mathrm{ml}$ of wash buffer 3

- elute in 3 fractions of $100 \mu 1$ of $2.5 \mathrm{mM}$ desthiobiotin

\subsubsection{Purification with chitin Beads:}

\subsubsection{Wash buffer 1: $10 \mathrm{mM}$ phosphate buffer}

- $\quad$ 10X Sodium Phosphate Buffer (100mM Sodium Phosphate pH7), 1L

- $\quad 500 \mathrm{mls}$ of $1 \mathrm{M}$ Dibasic Sodium Phosphate $\left(\mathrm{Na}_{2} \mathrm{HPO}_{4}\right) \mathrm{Add} 71 \mathrm{~g}$ and make up to $500 \mathrm{mls}$ with $\mathrm{dH}_{2} \mathrm{O} 500 \mathrm{mls}$ of $1 \mathrm{M}$ Monobasic Sodium Phosphate $\left(\mathrm{NaH}_{2} \mathrm{PO}_{4}\right)$

- $\quad$ Add 69g and make up to 500mls with $\mathrm{dH}_{2} \mathrm{O}$

- $\quad$ Add 50mls of 1M Dibasic Sodium Phosphate to 50mls of 1M Monobasic Sodium Phosphate and make up to $1 \mathrm{~L}$ with $\mathrm{dH}_{2} 0$.

1X Sodium Phosphate Buffer (10mM Sodium Phosphate pH7), 1L 
- $\quad$ Add $100 \mathrm{mls}$ of $10 \mathrm{X}$ Sodium Phosphate Buffer to $900 \mathrm{mls}$ of $\mathrm{dH}_{2} \mathrm{O}$

\subsubsection{Wash buffer 2: $10 \mathrm{mM}$ phosphate buffer $+1 \mathrm{M} \mathrm{NaCl}$}

$1 \mathrm{X}$ Sodium Phosphate Buffer $+1 \mathrm{M} \mathrm{NaCl}(10 \mathrm{mM}$ Sodium Phosphate $\mathrm{pH} 7), 500 \mathrm{ml}$

- $\quad$ 50ml of 10X Sodium Phosphate Buffer

$-\quad 29.2 \mathrm{~g}$ of $\mathrm{NaCl}$

- $\quad$ Make up to $500 \mathrm{ml}$ with $\mathrm{dH}_{2} \mathrm{O}$

\subsubsection{Wash buffer 3: 0.1 M acetic acid}

$[$ stock $]=17.4 \mathrm{~N}=17.4 \mathrm{M}$

- $\quad 11.4 \mathrm{ul}$ conc acetic acid + H2O till $20 \mathrm{ml}$

- $\quad$ Wash buffer 4: 1x PBS

\subsection{Purification protocol:}

- $\quad$ wash beads with $2 \mathrm{ml}$ of wash buffer 1

- $\quad$ incubate sample and beads for 1 hour rotating at $4{ }^{\circ} \mathrm{C}$

- $\quad$ place beads in column and let sample flow by gravity

- $\quad$ wash beads $2 \mathrm{ml}$ of wash buffer 1

- $\quad$ wash beads $2 \mathrm{ml}$ of wash buffer 2

- $\quad$ wash beads $2 \mathrm{ml}$ of wash buffer 3

- $\quad$ wash beads $3 \mathrm{ml}$ of wash buffer 4

- $\quad$ add $100 \mu \mathrm{l}$ of loading dye to the beads and remove them out of the column by spinning

\subsubsection{Purification with Amylose beads:}

Add to the sample the same salts in the same concentrations as in the wash buffer. 
2.19.4.1 Wash buffer

$20 \mathrm{mM}$ Tris- $\mathrm{HC}(\mathrm{pH}=7.4)$

$0.2 \mathrm{M} \mathrm{NaCl}$

$10 \mathrm{mM} \beta$-mercaptoethanol (added right before use)

\subsection{Purification protocol:}

- wash beads 2 times with $1 \mathrm{ml}$ of wash buffer 1

- incubate sample and beads for 1 hour rotating at $4{ }^{\circ} \mathrm{C}$

- place beads in column and let sample flow by gravity

- wash the beads with $3 \mathrm{ml}$ of wash buffer

- elute with 3 fractions of elution buffer $(100 \mu l)$

\subsubsection{Biological LP purification:}

Used for Ni-NTA purification of His tagged proteins:

Consumables:

Econo-columns (BioRad)

Ni-NTA beads (BIORAD); volume used as indicated by manufacturer.

Buffers specific for Ni purification (see section buffers)

Procedure:

The flow will always be kept at $1 \mathrm{ml} / \mathrm{min}$

Salts are added to the sample in such a way the final concentration will be the same as in the washing buffer. The sample will then beintroduced on to the column. Washing will be performed until the absorbance $(280 \mathrm{~nm})$ reaches the base line and at that point elution will be started, being 
a gradient of elution buffer form $0-100 \%$ in 35 min. Elution fractions will be collected by the autosampler in $1.5 \mathrm{ml}$ microcentrifuge tubes, being each fraction of $500 \mu 1$.

\subsubsection{SV5 purification using protein A tips (Phynexus):}

Consumables:

Protein A tips (Phynexus, PTR 92-05-01)

PhyTip ME 200 Purification System (Phynexus, PHR 12-020-00)

anti-SV5 antibody

Buffers from the protein A purification kit: purification and enrichment

PCR plates

PBS-T

PBS-LT

Procedure:

Each sample and washing buffer was placed in a well of a PCR plate. Volume of washing buffer was $200 \mu \mathrm{l}$ in each well. Volume of elution buffer was $40 \mu 1$. First well contained the -SV5 mixture to be captured by the protein A tips.

Single purification with a-SV5 was performed by running the step \#4 of capture over the initial sample (mixture of scFvs and cell extract with antigens). Double purification was done by running this step over each of the strep-tactin eluted fractions.

The programs used are the ones designed by the manufacturer for the 200 protein A tips. For the elution the capture program was modified to aspirate $30 \mu 1$.

protocol " $200+$ protein A" 
capture: $200+$ default proA capture

wash 1:200+ default proA Wash 1

wash 2: $200+$ default proA Wash 2

elution: $200+$ default proA enrichment

Prepare the anti-SV5 sample to coat the tips:

$2 \mu 1$ of anti-SV5 $(5 \mu \mathrm{g} / \mu \mathrm{l})+128 \mu \mathrm{l}$ 1xPB

$40 \mu 1$ of $5 \mathrm{x}$ capture buffer (PBS)

$30 \mu 1$ deionized water

1-2x "capture: $200+$ default proA capture "- on the a-SV5 sample

2-1x wash 1: 200+ default proA Wash 1- purification buffer

3-1x wash 2: 200+ default proA Wash 2- purification buffer

4- $2 \mathrm{x}$ "capture: $200+$ default proA capture “- on each of the strep-tactin eluted fractions

5- 1x wash 1:200+ default proA Wash 1- PBS-T

6- 1x wash 2: 200+ default proA Wash 2- PBS-T

7- 1x wash 1: 200+ default proA Wash 1- PBS-LT

8-1x wash 2: 200+ default proA Wash 2- PBS-LT

9- 3x elution: $200+$ default proA enrichment

10- Add $5 \mu 1$ of neutralization buffer 


\subsection{Preparation of Shewanella oneidensis MR-1 cell pellet}

(By Shu-mei W. Li on behalf of Dr. James K. Fredrickson)

\section{Culture medium:}

Bacto tryptic soy broth w/o dextrose (TSB) (Ref \#286220, Becton, Dickson and Company, Sparks, MD)

Pre-culture:

- $10 \mathrm{~mL}$ TSB, inoculate with $0.1 \mathrm{~mL}$ cryopreserved material of S. oneidensis MR-1 (stored at $-80^{\circ} \mathrm{C}$ freezer).

- Incubation: $30{ }^{\circ} \mathrm{C}, 150 \mathrm{rpm}$ gyratory shaking, overnight (18-20 h)

Culture:

- $500 \mathrm{~mL}$ TSB (in a $1000 \mathrm{~mL}$ size flask) inoculate with $5.0 \mathrm{~mL}$ pre-culture

- Incubation: $30^{\circ} \mathrm{C}, 150 \mathrm{rpm}$ gyratory shaking, overnight $(18-20 \mathrm{~h})$

Harvesting:

- 5,000 g x 10 min at $4{ }^{\circ} \mathrm{C}$, decant supernatant, and store the pellet at $-80{ }^{\circ} \mathrm{C}$ until use.

- Optional: re-suspend pellet with suitable volume of phosphate-buffered-saline (PBS)* and centrifuged again. Decant supernatant and store the pellet at $-80{ }^{\circ} \mathrm{C}$ until use.

*Phosphate-Buffered-Saline (PBS): per liter

$\begin{array}{ll}\mathrm{Na}_{2} \mathrm{HPO}_{4} .7 \mathrm{H}_{2} \mathrm{O} & 2.22 \mathrm{~g} \\ \mathrm{NaH}_{2} \mathrm{PO}_{4} . \mathrm{H}_{2} \mathrm{O} & 0.223 \mathrm{~g} \\ \mathrm{NaCl} & 8.5 \mathrm{~g} \\ \mathrm{pH} \text { to } 7.0 & \end{array}$




\subsection{Kcoil-Alexa488 synthesis}

\subsubsection{K coil sequence:}

KVSALKEKVSALKEKVSALKEKVSALKEKVSALKEC

\subsubsection{Synthesis:}

Peptide was synthesized on an ABI 433A synthesizer using standard FastMoc Chemistry in $0.25 \mathrm{mmol}$ scale and double coupling of all residues. Reagents, solvents and aminoacids were purchased from ABI, Fisher, Aldrich and NovaBiochem; all in in synthesis grade. The resin was a Fmoc Cys Cl-trityl resin at $0.4 \mathrm{mmol} / \mathrm{g}$ loading from ACT.

\subsubsection{Deprotection/purification:}

1. Add $10 \mathrm{~mL}$ of a solution of $92 \%$ TFA (sequencing grade), water $5 \%$ and $3 \%$

Triisopropylsilane to $1 \mathrm{~g}$ resin. Incubate the resin for $8 \mathrm{~h}$ at $\mathrm{rt}$ on an orbital shaker under Argon.

2. Precipitate peptide into $25 \mathrm{~mL}$ ice cold ether

3. Centrifuge, discard supernatant ether, then add fresh ether, re-suspend and centrifuge repeat his wash step 3 times.

4. Dry peptide under vacuum.

5. Redissolve in water with $0.1 \%$ TFA

6. HPLC purification of the peptide on a semi preparative Waters 600 system using a Waters Symmetry column C18, 300 A (this is Angstrom), $7 \mu \mathrm{m}, 19 \times 150 \mathrm{~mm}$ at 20 
$\mathrm{mL} /$ min running a gradient from $92 \% \mathrm{ACN} / 8 \% \mathrm{H} 2 \mathrm{O} / 0.1 \%$ TFA to $50 \% \mathrm{ACN} / 50 \%$ $\mathrm{H} 2 \mathrm{O} / 0.1 \%$ TFA with a linear gradient in $12 \mathrm{~min}$ (rt 10.42).

7. Remove the $\mathrm{ACN}$ on a rotary evaporator and freeze dry the peptide on a lyophilizer.

Peptide when stored under argon can be used directly for derivatization of the Cys thiol.

\subsubsection{Regeneration of the thiol after extended storage:}

Using ReduceImm columns (Pierce/ immobilized and activated DTT on agarose) samples for direct attachment of a thiol reactive dye can be prepared following the manufacturer's protocol.

\subsubsection{Attachment of the Alexa fluor:}

Label the reduced peptide (from HPLC purification/ or from RedImm) with Alexa Fluor 488 (C5 maleimide). Ratio Alexa: peptide of 1:2 at pH 7.8 (PBS or RedImm buffer). Purify the Alexa labeled peptide from unreacted Alexa fluor and excess peptide by gel filtation (Bio-Gel P-6)

Larger peptide quantities were labeled as described above but purified on a semi preparative Waters 600 system using a Waters Symmetry column C18, $300 \AA \AA, 7 \mu \mathrm{m}$, $19 \times 150 \mathrm{~mm}$ at $20 \mathrm{~mL} / \mathrm{min}$ running a gradient from $94 \% \mathrm{ACN} / 6 \% \mathrm{H} 2 \mathrm{O} / 0.1 \% \mathrm{TFA}$ to $60 \% \mathrm{ACN} / 40 \% \mathrm{H} 2 \mathrm{O} / 0.1 \%$ TFA with a step gradient (5) in $15 \mathrm{~min}$ (rt $17 \mathrm{~min}$ ).

\subsubsection{Peptide identification:}

Peptides were identified on a Thermo LCQ ESI mass spectrometer in the positive mode with $0.1 \%$ Formic acid / $\mathrm{H} 2 \mathrm{O}$ and showed peaks at $\mathrm{M}+2$ and higher ionization. The peptides show a high portion of $\mathrm{Na}$ and $\mathrm{K}$ ion peaks in the ESI which are often observed as counterions for Alexa fluorophores. 
${ }^{1}$ Studier, F. W.; Moffatt, B. A., Use of bacteriophage T7 RNA polymerase to direct selective high-level expression of cloned genes. J Mol Biol 1986, 189, (1), 113-30. 


\section{Chapter 3. Vector construction and testing}

\subsection{Introduction}

This chapter describes the construction of a set of vectors, pRosa, and demonstrates their functionality. The aim of the thesis is to develop an alternate method for immunoprecipitation of protein complexes. This method is based on the use of single chain antibodies (scFvs) to interact with the target protein and pull down the protein complexes formed.

The pRosa series of vectors were constructed to allow the directional cloning of scFvs selected from a pDAN5 library selection ${ }^{1}$. These multifunctional expression vectors provided the possibility of introduction of tags to be expressed as single chain fusions. A set of affinity tags, CBP (Calmodulin binding protein), GST (Glutathione-Stransferase), strep tagII, CBD (Chitin Binding Domain) and MBP (Maltose Binding Protein), were chosen and studied as fusions to the amino terminus of the scFv. These tags were introduced to be used as purification handles of protein complexes bound to the scFvs.

ScFv-tag fusions were checked for expression and interaction with the antigen, with the conclusion that the presence of strep tag II, CBD and MBP do not have a negative effect on either. Purification experiments demonstrated the use of the tags as purification handles however strep tagII was the one selected for immunoprecipitation experiments due to the mild washes and elution needed. 


\subsection{Amplification of affinity tags:}

The tags used in this study include the Strep tagII (strep), calmodulin binding protein (CBP), chitin binding domain (CBD), glutathione-S-transferase (GST) and maltose binding protein (MBP). These tags will be labeled "immunoprecipitation tags" (I.P. tags) during this study to differentiate them from other tags already present in the vectors.

The tags were cloned by PCR using primers containing specific restriction enzyme (RE) sites and different sources as templates.

The destination vector was designed with the flexibility of being able to clone tags at either the amino $(\mathrm{N})$ or carboxy $(\mathrm{C})$ termini of the single chain. However, the studies were only completed with tags fused to the $\mathrm{N}$ terminus.

Primers were designed to introduce SfiI and AgeI restriction sites flanking the tags for cloning at the amino terminus of the $\mathrm{scFv}$. The primers were also designed to include an additional non-encoding $33 \mathrm{bp}$ sequence to prevent steric hinderence at the protein level, due to the location of the $\mathrm{N}$ terminus at the antigen interaction site of the scFv. The genes encoding the tags MBP, GST, CBD, were amplified from the commercial vectors (Refer to

1). In contrast, the genes encoding the tags StrepII and CBP were created by linker annealing, due to their small sizes (91 and 142 bp respectively). 


\begin{tabular}{|c|c|c|c|c|c|}
\hline Origin & from & TAG & R.S. (5) & SIZE(bp) & $\begin{array}{c}\text { expressed protein } \\
(\mathbf{k D})\end{array}$ \\
\hline & & flag & SfiI/AgeI & 21 & 31.8 \\
\hline Primers & Primers & Strep tag II & SfiI/AgeI & 91 & 33 \\
\hline Primers & Primers & CBP $(1)$ & SfiI/AgeI & 142 & 35 \\
\hline PTYB1 & NEB, Beverly, Mass & Chitin (2) & SfiI/AgeI & 222 & 37.5 \\
\hline PET-42 & EMD biosciences & GST (3) & SfiI/AgeI & 723 & 57.4 \\
\hline Pmal-p2x & NEB, Beverly, Mass & MBP (4) & SfiI/AgeI & 1167 & 72.2 \\
\hline
\end{tabular}
(1) Calmodulin binding protein
(2) Chitin binding domain
(3) Glutathione-S-transferase
(4) maltose binding protein

(5) Restriction sites

Table 3-1: Cloned tags information. Including a scFv of 31 kD.

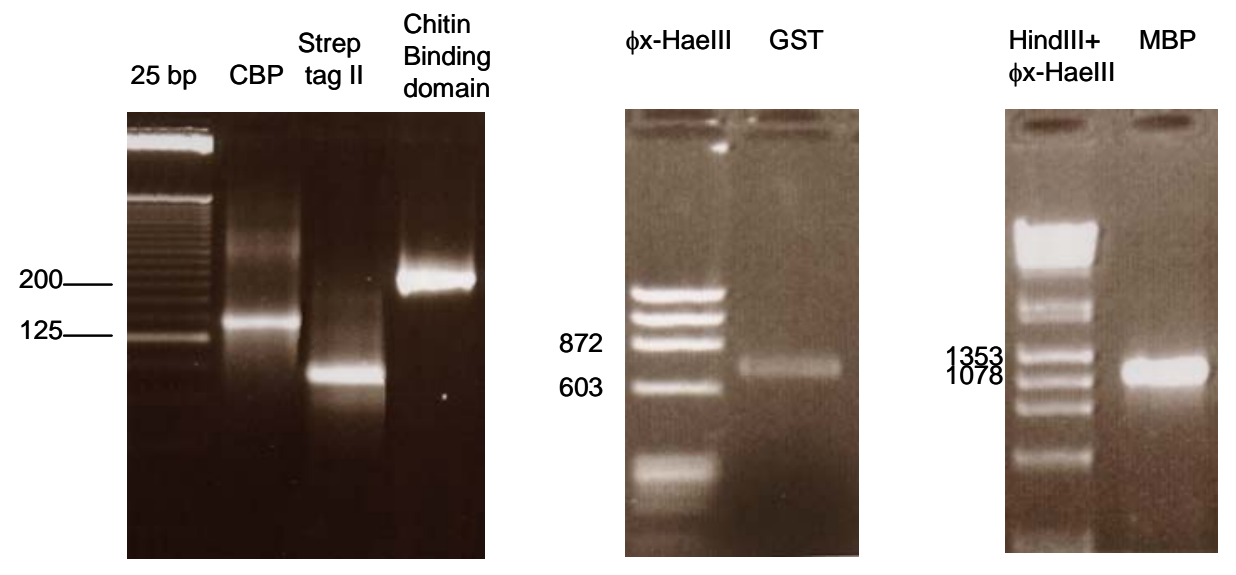

Figure 3-1. Amplification of the tags by pcr; CBP, Strep-tag II, CBD, GST and MBP

The PCR reaction and linker annealing amplification method is described in chapter 2: PCR. PCR amplification produced DNA fragments of the expected size in all cases (refer to Table 3-1). Following gel excision, the DNA was extracted (QIAGEN gel extraction kit), digested with restriction enzymes AgeI and SfiI, and purified in preparation for subsequent cloning steps. The prepared DNA was then stored at $-20^{\circ} \mathrm{C}$. 


\subsection{Creation of expression vector for scFv-tag fusions (pRosa6):}

The expression vector, pRosa6, was created for compatible sub-cloning of DNA encoding single chain antibodies originating from the pDAN5 library. In addition, the vector was designed to include one or more affinity tags to aid protein solubility, expression and purification.

The commercial vector pET28b ${ }^{2}$ (Novagen) was modified by introducing restriction enzyme sites to allow directional cloning of genes encoding both the tags and single chain antibodies. The expression vector, pRosa6, was created by sequential modifications of the display vector, pDAN5, and subsequent amalgamation with pET28. A theoretical scheme of the cloning is shown (Refer to Figure 3-2). All the preliminary experiments were performed using $3 \mathrm{~A} \mathrm{scFv}$ as a model single chain. $3 \mathrm{~A}$ is a scFv that recognizes the SNV-N (sin nombre virus nucleocapsid) protein ${ }^{3}$. 
- Introduction of restrictions sites (R.S.) SfiI, AgeI, NcoI and a flag gene.

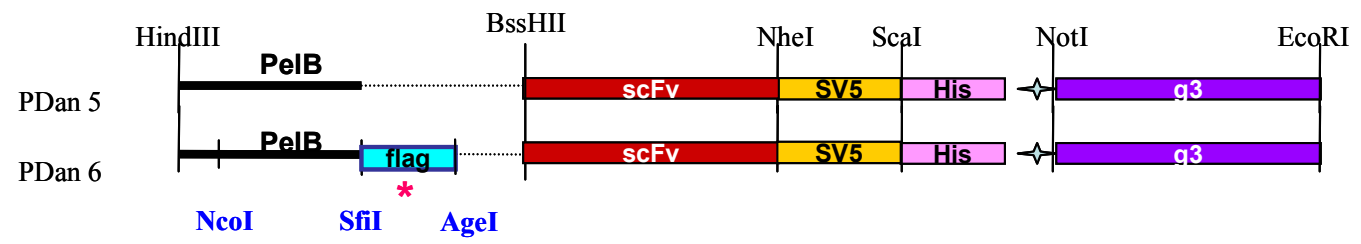

* flag epitope will remain attached to the scFv after cleavage of the pel $\mathrm{b}$ leader in the periplasmic membrane

-Cloning into the $\mathrm{pET}-28$ vector

NcoI/EcoRI insert from pDan6

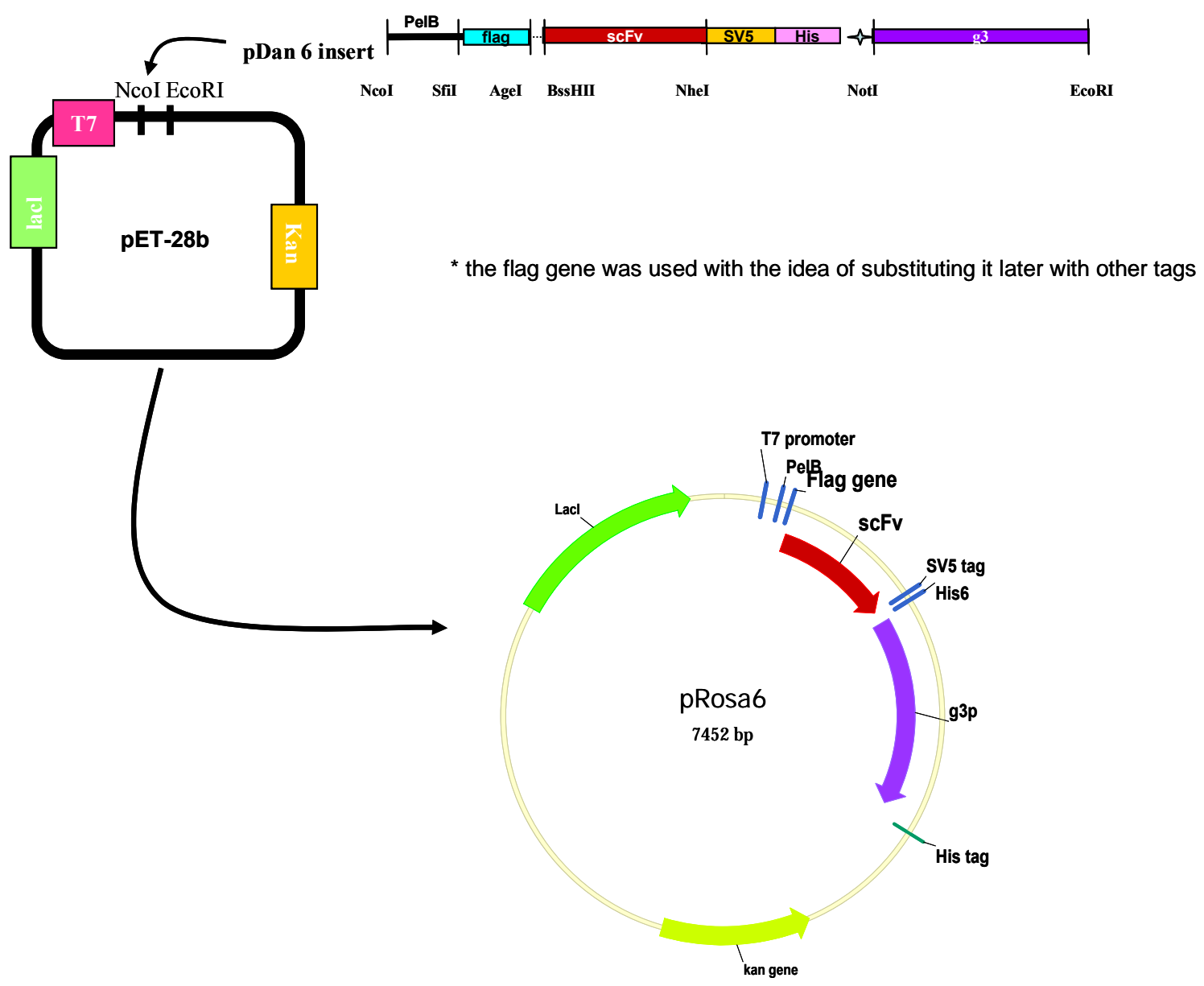

Figure 3-2. Schematic of the cloning process to engineer pRosa6. 


\subsubsection{Cloning of pDAN6:}

The first step in the creation of pRosa6 was the introduction of a FLAG tag gene and restriction enzyme sites SfiI, AgeI and NcoI into the pDAN5 vector.

This gene, flanked by AgeI and SfiI restriction sites, was introduced upstream of the single chain with the plan of substituting it later for different tags. An NcoI restriction site was also introduced for future cloning steps. This created the pDAN6 vector.

The sequence encoding the FLAG tag was PCR amplified from the pDAN11 vector ${ }^{4}$ using the primers pelBNcoI/pelBsfiI. Following PCR amplification (Chapter 2, PCR), the 143 bp DNA product was digested with BssHII and HindIII restriction enzymes and was cloned directionally into BssHII/HindIII digested pDAN5 vector.

Following ligation and transformation, the DNA produced from six transformants was digested with a range of restriction enzymes to obtain a fingerprint for each clone (Refer to Figure 3-3) 

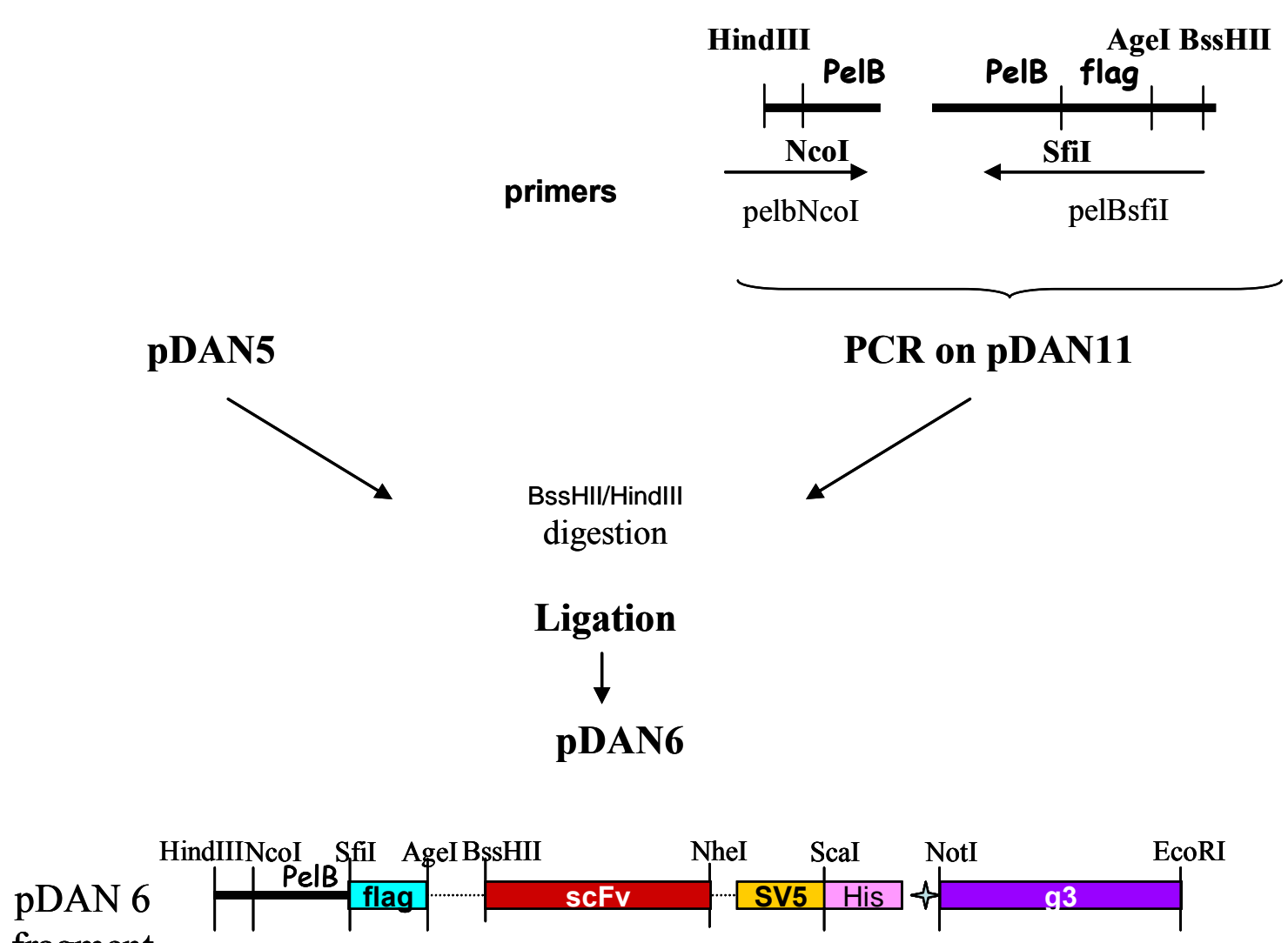

fragment

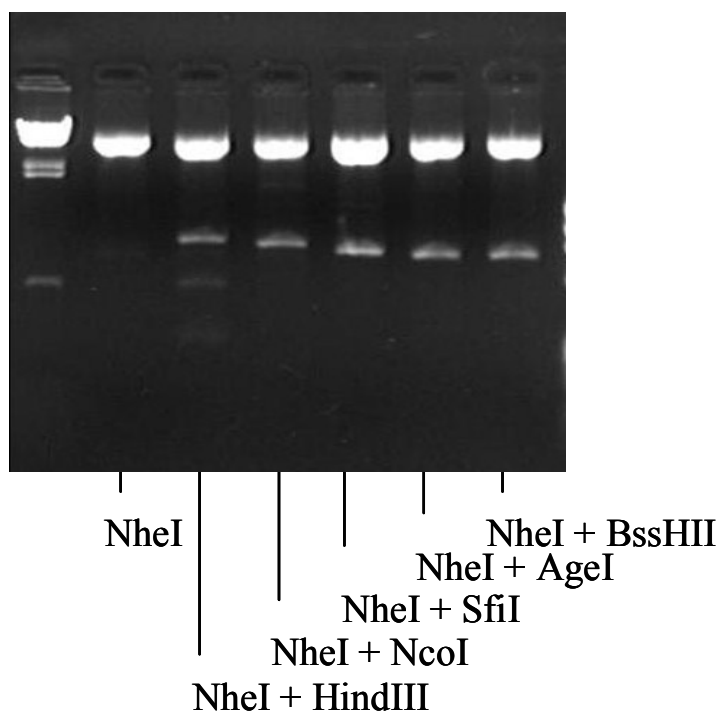

Figure 3-3. Schematic of the creation of pRosa6 and fingerprint 


\subsubsection{Cloning of pRosa6:}

The expression vector pRosa6, was constructed by directionally cloning the $2.1 \mathrm{kbp}$ NcoI/EcoRI fragment from pDAN6 into NcoI/EcoRI digested pET28b (Refer to Figure 3-4). To construct the pRosa6-3A vector, the pDAN6 vector was digested with NcoI and EcoRI restriction enzymes (R.E.). The 2.1 Kbp fragment was recovered and sub-cloned into NcoI/EcoRI restricted pET-28b to create pRosa6. The pRosa6 vector was characterized by fingerprinting with a panel of restriction enzymes as shown in Figure $3-4)$.

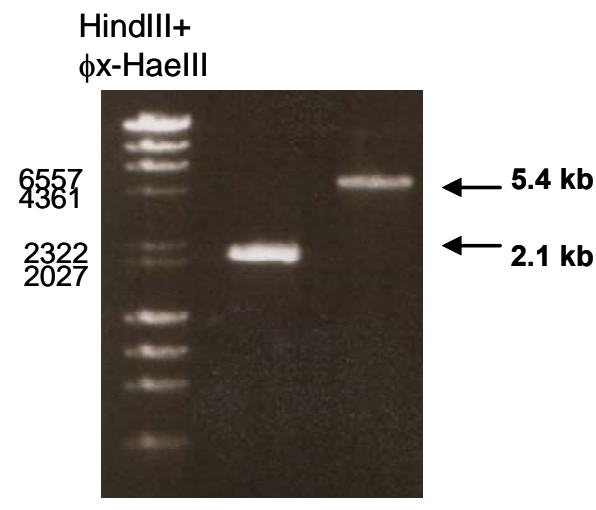

(2) (1)

Figure 3-4. Purified fragments of pET-28 (1) and pDAN6 (2) vectors, digested with EcoRI and NcoI. Ligation of both fragments produce pRosa6 


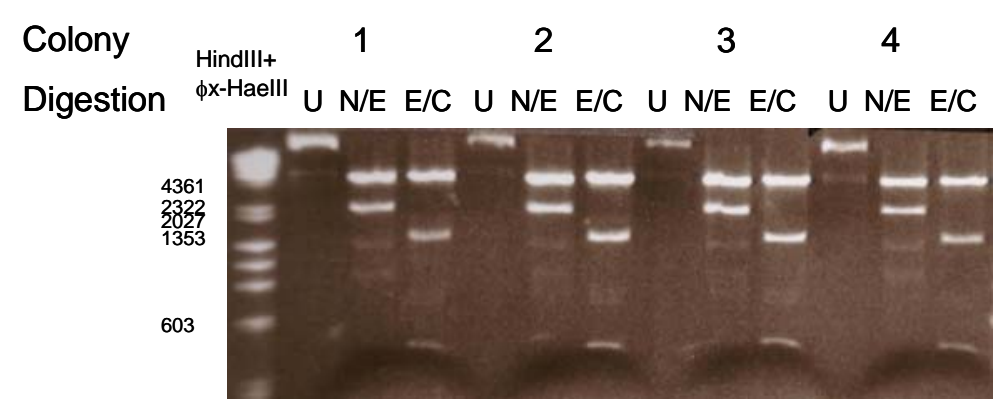

Figure 3-5. Fingerprint of 4 colonies from pRosa6 ligation. Undigested (U), NcoI and EcoRI digestion $(\mathrm{N} / \mathrm{E})$, EcoRI and ClaI digestion (E/C)

Digestion with NcoI/EcoRI (N/E), produced two fragments of 2187 and $5265 \mathrm{bp}$.

Digestion with EcoRI and ClaI (E/C), produced fragments of 366, 1446 and $5640 \mathrm{bp}$. Restriction enzyme analysis demonstrated that all cloned features of pRosa6 were present. Sequence analysis confirmed the DNA sequences were correct and in frame.

\subsection{Cloning of the Tags into pRosa6:}

A panel of expression vectors was produced by interchanging the Flag tag gene of PRosa6 with the I.P. tags described in section 3.2. The DNA fragments encoding the tags (Refer to section 3.2), previously digested with SfiI and AgeI restriction enzymes, were directionally ligated into the $7.4 \mathrm{~kb}$ AgeI/SfiI digested pRosa6. The resulting clones were checked for the presence of cloned DNA fragments via restriction enzyme digestion with NcoI and AgeI. The expected DNA fragments are described in Table 3-2 and the digestions shown in Table 3-2.

\begin{tabular}{|c|c|}
\hline & Ncol/Agel fragments (bp) \\
\hline pRosa6-scFv-Strep & 7369,131 \\
\hline pRosa6-scFv-CBP & 7369,185 \\
\hline pRosa6-scFv-Chit & $7369, \mathbf{2 6 0}$ \\
\hline pRosa6-scFv-GST & 7369,761 \\
\hline pRosa6-scFv-MBP & $7369,1032,173$ \\
\hline
\end{tabular}

Table 3-2: Fragments after NcoI and AgeI digestion 


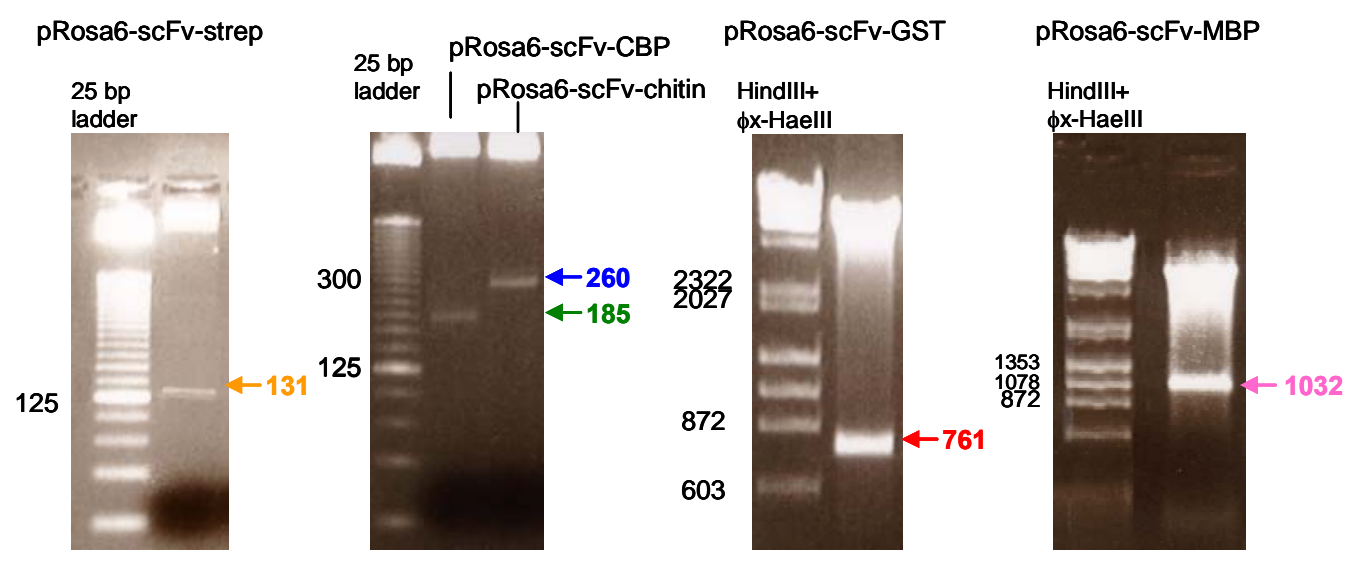

Figure 3-6. Digestion of pRosa6-scFv-CBP, pRosa6-scFv -chitin, pRosa6-scFv -GST, pRosa6-scFv MBP and pRosa6-scFv -strep with NcoI and AgeI RE.

ELISA (Enzyme Linked Immunosorbant Assay) was used to demonstrate that the presence of tags did not affect the ability of the single chain to bind its antigen. Expression of the right size single chain fused to tags was checked by Western blot.

When examined by western blotting (Refer to Figure 3-7) all the tags, except GST gave bands of the appropriate size. However, in addition to GST, CBP also gave very low expression levels (Refer to Figure 3-7), and as a result, further work with these two tags was discontinued. All tags gave two additional prominent bands at 17-19 kD. Similar bands have been seen with $\mathrm{scFv}$ without fusion tags, and are thought to represent the $\mathrm{VH}$ chain fused to the SV5 tag. 


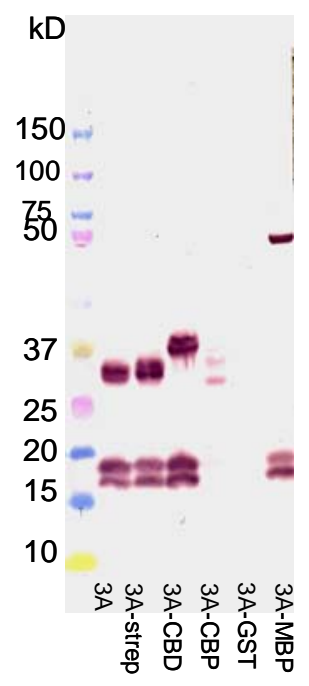

SV5 ELISA on scFvs-tag proteins expressed from pRosa6 vectors

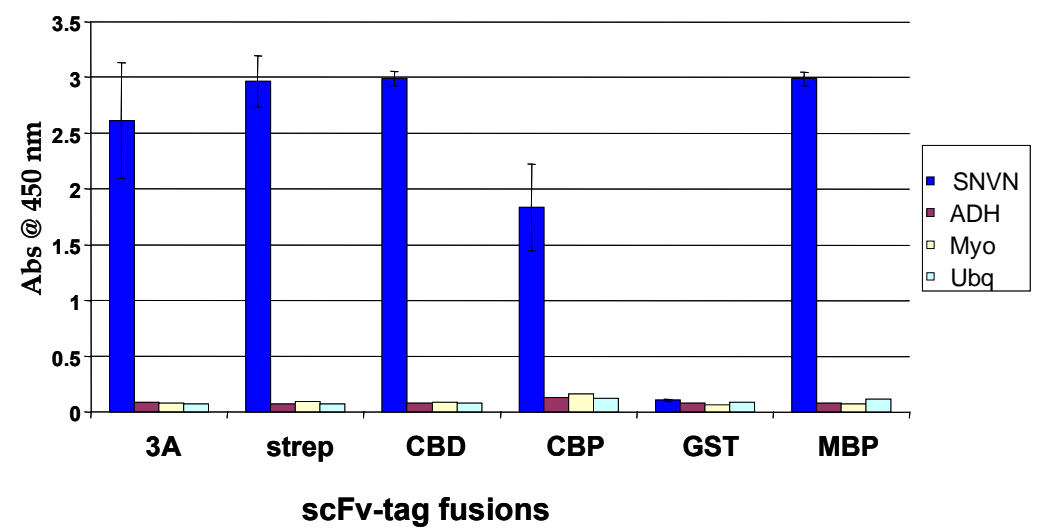

Figure 3-7. Expression of pRosa6 vectors with different tags. Samples analyzed by SV5 Western blot and ELISA. Refer to Table 1 for sizes

$\mathrm{ScFv}$ fusions to Strep tagII, CBD and MBP expressed from pRosa6 vectors gave good ELISA signals comparable to the ones obtained from the non tagged vector. They also showed specificity for the selected antigen. CBD and GST fusions gave little or no signal corresponding to the low expression levels found on the Western blot. 


\subsection{Evolution of the pRosa 6 vector:}

The expression vector pRosa6 underwent different modifications in order to make it more versatile and to improve the quality of the final expressed protein. A scheme provides information of the modifications and the engineered vectors.

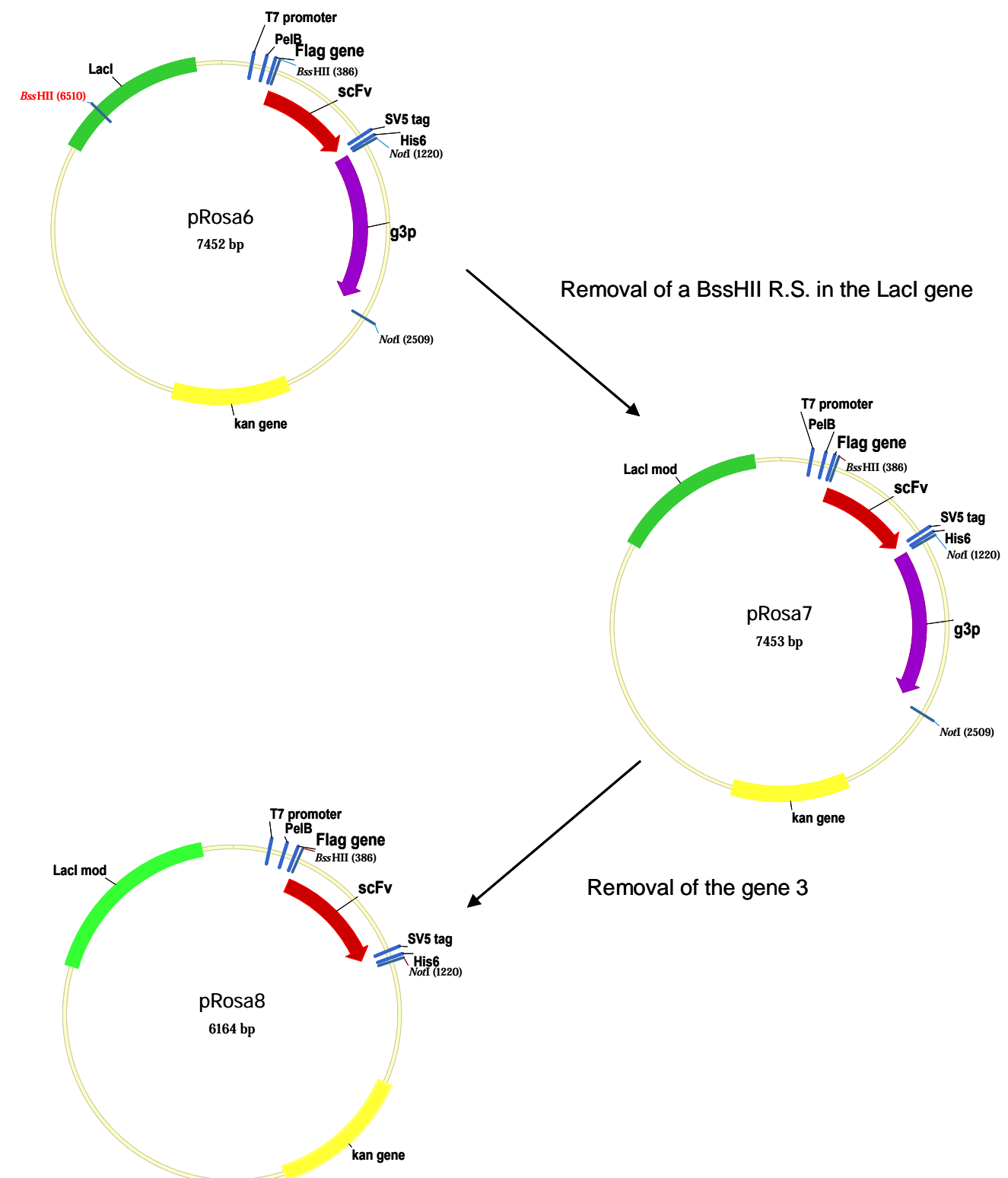

Figure 3-8. Evolution of the pRosa vectors 


\subsection{Creation of pRosa 7:}

In order to exchange single chains, a new modification needed to be done to the tagged expression vector pRosa6. The strategy for cloning scFvs into pRosa6 involved digestion with NheI and BssHII restriction enzymes. However, a second BssHII site present in the LacI gene, needed to be removed before cloning was possible (Refer to Figure 3-8). Mutagenesis was the method chosen to remove it, and was performed using the GeneTailor ${ }^{\mathrm{TM}}$ Site-Directed Mutagenesis System (Invitrogen). The primers, LacI and LacImut (Refer to chapter 2: Primers), were designed to introduce a base pair mutation at the LacI BssHII site so that the restriction site would disappear whilst not changing the amino acid composition.

\begin{tabular}{lll} 
& & \multicolumn{2}{c}{ BSSHII } \\
& & $\sim \sim \sim \sim \sim$ \\
GGA CTC GGT AAT GGC GCG CAT \\
CCT GAG CCA TTA CCG CGC GTA \\
Ser Glu Thr Ile Ala Arg Met
\end{tabular}

Mutated gene

$\begin{array}{llllll}\text { GGA } & \text { CTC } & \text { GGT } & \text { AAT } & \text { GGC ACG } & \text { CAT } \\ \text { CCT GAG CCA TTA CCG TGC GTA } \\ \text { Ser Glu Thr Ile Ala Arg Met }\end{array}$

Figure 3-9. Schematic of the mutation introduced to remove the BssHII site from the lacI gene.

The mutated clones were checked by digestion of the DNA with BssHII. The presence of two BssHII restriction sites in pRosa6 produced two fragments of 6124 and $1328 \mathrm{bp}$ while mutated vector is linearized. The new vector with mutated LacI gene was named pRosa7. 


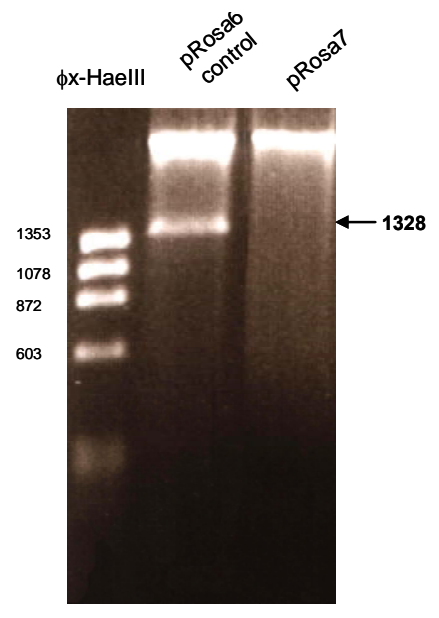

Figure 3-10. BssHII digestion of pRosa7 and pRosa6

All the tags were then transferred from pRosa6 into pRosa 7 by two different cloning strategies.
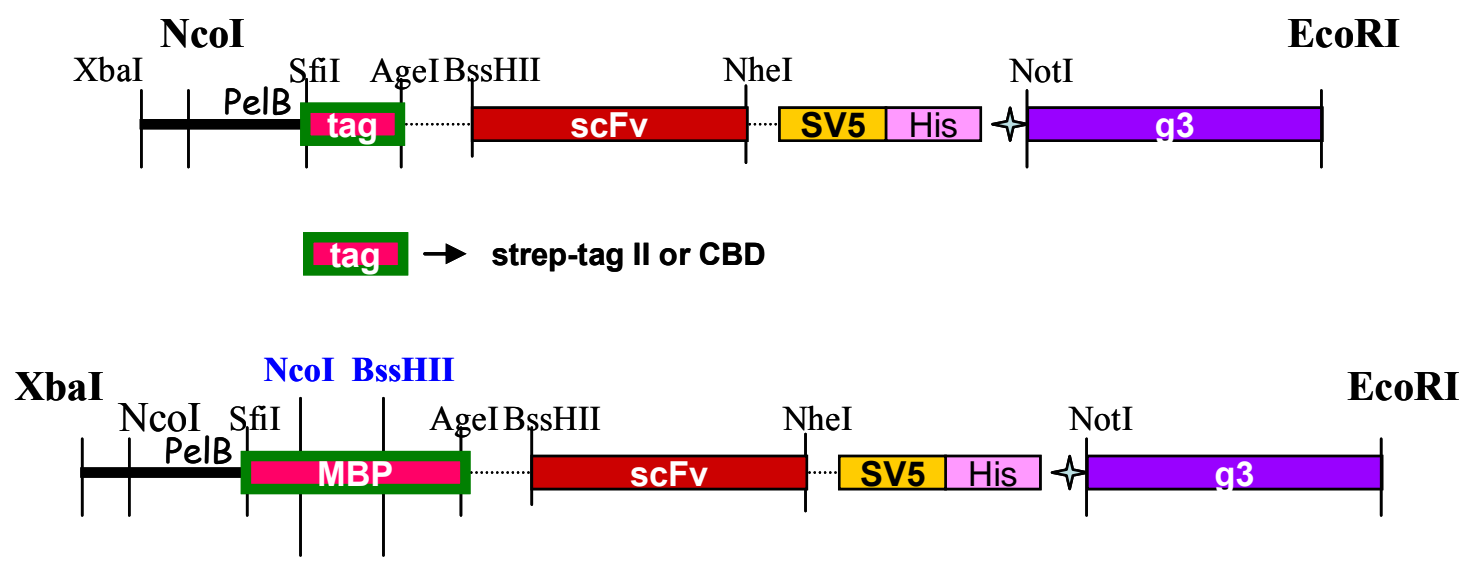

Figure 3-11. Diagram showing the extra R.S. found in the DNA encoding the MBP tag 
DNA encoding the Strep-tag II and the CBD were cloned into pRosa7 vector by digestion of the correspondent pRosa6-tag plasmids with NcoI and EcoRI restriction enzymes (Refer to Figure 3-11). The 2364 bp CBD fragment and 2235 bp strep tag NcoI/EcoRI fragments were cloned into NcoI/EcoRI digested pRosa7.

Due to the presence of an NcoI site inside of the gene encoding the MBP tag, a different cloning strategy was used. The $3348 \mathrm{bp} \mathrm{XbaI/EcoRI} \mathrm{fragment,} \mathrm{containing} \mathrm{the}$ MBP gene, was cloned into XbaI/EcoRI digested pRosa7 (Refer to Figure 3-11).

The resulting clones were characterized by restriction enzyme digestion, as shown in figures 3-12 and 3-13.
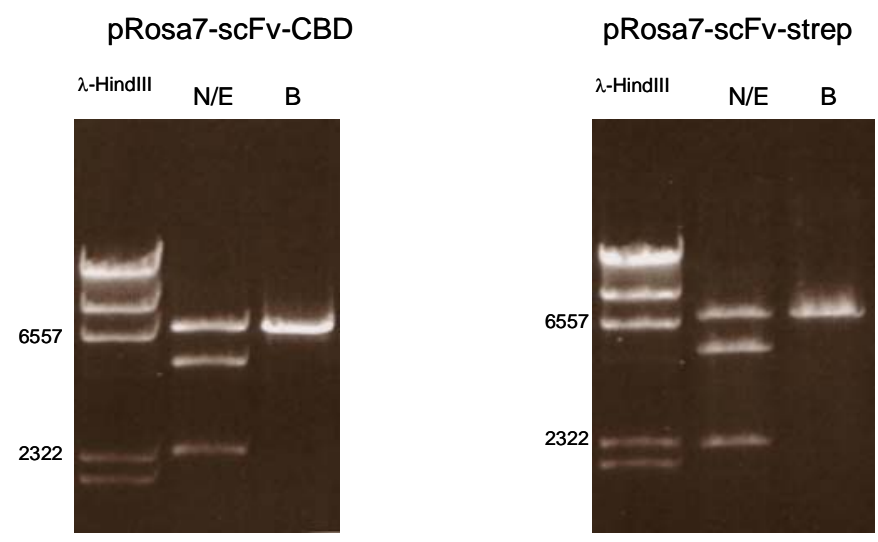

Figure 3-12. pRosa7-scFv-chitin and pRosa7-scFv-strep vectors checked by digestion with NcoI/EcoRI (N/E) and with BssHII (B) 
Figure 3-13. pRosa7-scFv-MBP vector checked by digestion with XbaI/EcoRI (X/E)

With the pRosa7 vectors engineered to contain three different I.P. tags, a new mutation had to be done to the pRosa7-scFv-MBP vector in order to be able to switch single chains using BssHII and NheI restriction sites The pRosa7-scFv-MBP vector gene contained a second BssHII restriction site within the MBP gene that interfered with the cloning plan of new single chains (Refer to Figure 3-11). This site was removed by mutagenesis using the GeneTailor ${ }^{\mathrm{TM}}$ Site-Directed Mutagenesis System (Invitrogen), with the primers MBPmutg5 and MBPmutgnew3 (Chapter2, primers)
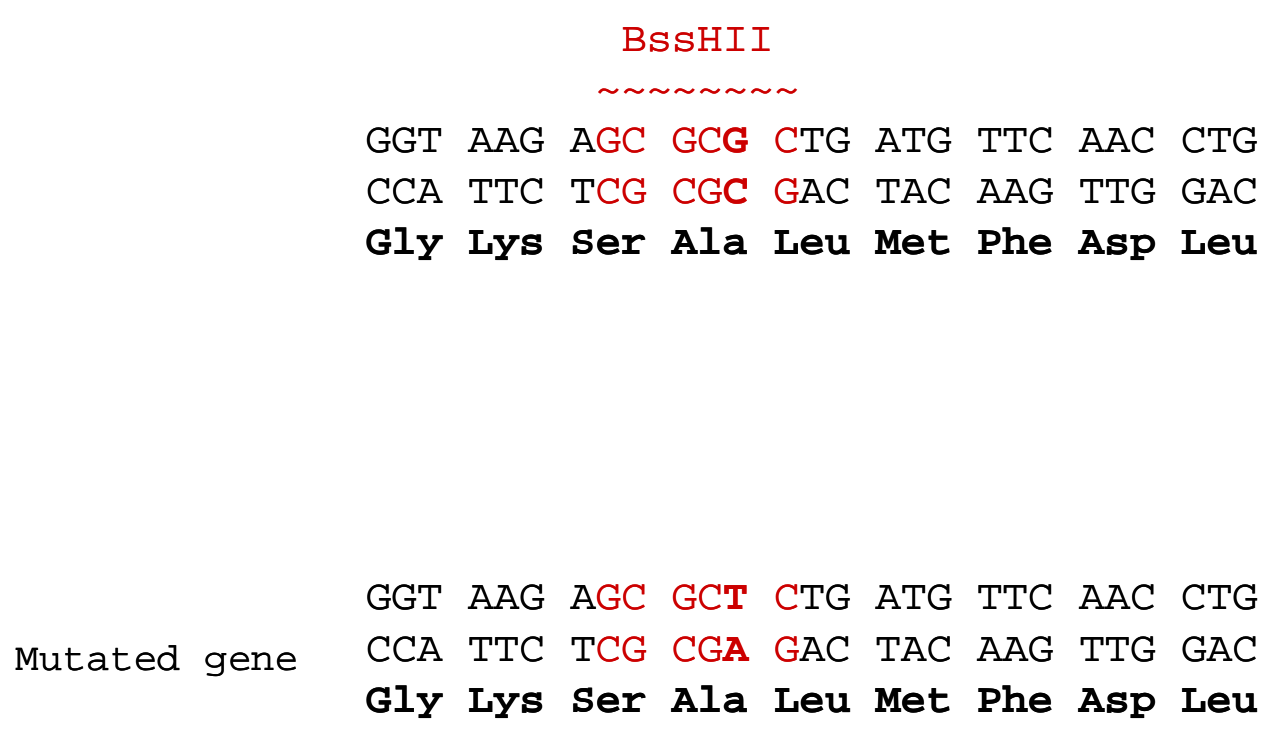

Figure 3-14. Mutagenesis on the MBP gene to remove the BssHII restriction site 
The DNA of the mutagenized clones was checked by restriction enzyme digestion with BssHII. The non-mutated, containing two BssHII sites produced a fragment of 705 bp while the mutated ones, containing only one BssHII site, linearized (Refer to Figure $3-15)$.

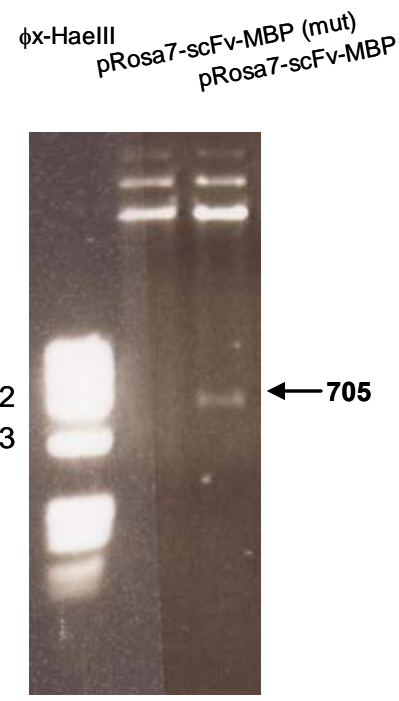

Figure 3-15. BssHII digestion in mutated pRosa7-scFv-MBP (mut), and non mutated pRosa7-scFvMBP

\subsubsection{Cloning of scFvs into the pRosa7 vector:}

Three different single chains recognizing three different targets were used to check the influence of the tags in the new expression vector D1.3 ${ }^{5}, 4 \mathrm{M} 5.3^{6}$, and G4 (selected against TB protein RV2607, Refer to Chapter 4).

\begin{tabular}{|c|c|c|}
\hline scFvs & Antigen & from \\
\hline D1.3 & Lysozyme & mouse monoclonal \\
\hline G4 & RV2607 (1) & human from scFv library \\
\hline 4M5.3 & Fluorescein & mouse monoclonal and yeast display \\
\hline
\end{tabular}

(1) TB protein

Table 3-3: Single chains information 
The 728 and 770bp BssHII/NheI fragments corresponding to the D1.3 and G4 single chain genes respectively, were sub-cloned from pDAN5 into the BssHII/NheI digested pRosa7 vectors. In contrast, the single chain 4M5.3 was PCR amplified from pDAN1 $11^{4}$ using Fwdantifluo and Revantifluo primers (Chapter 2: Primers). The PCR product was then digested with BssHII and XbaI restriction enzymesand the resulting 770 bp fragment was cloned into the BssHII/NheI digested pRosa7 vectors (digestion with XbaI and NheI restriction enzymes produces fragments with compatible overhangs).

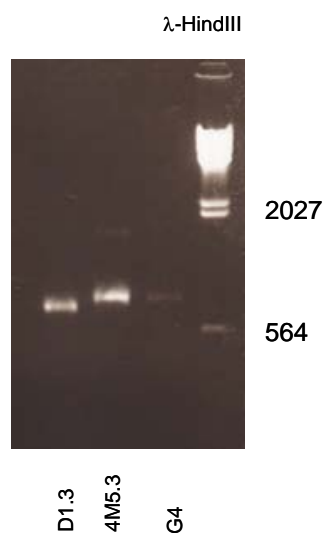

Figure 3-16. D.13 and G4 single chains digested with NheI and BssHII R.E; $\alpha$-FITC single chain digested with XbaI and NheI R.E

Each DNA clone was sequenced with the primers $\mathrm{pDpH5}$ ' and $\mathrm{pDpH} 3$ ', and in the case of the plasmids with MBP with also the MBP5 primer. The protein produced by each pRosa7-scFv-tag clone, was expressed. The proteins recovered within the periplasmic extract were analyzed by ELISA to determine interaction with the appropriate antigen (Refer to Figure 3-17). The expressed proteins were also analyzed by Western Blot analysis, to check the size and stability of the proteins. 
1)

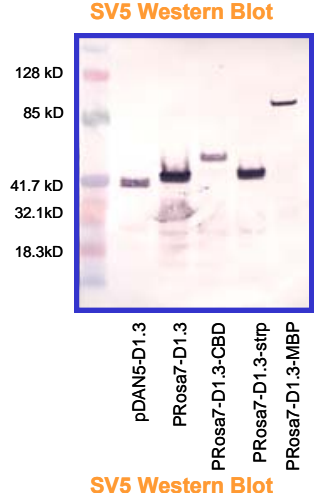

1)

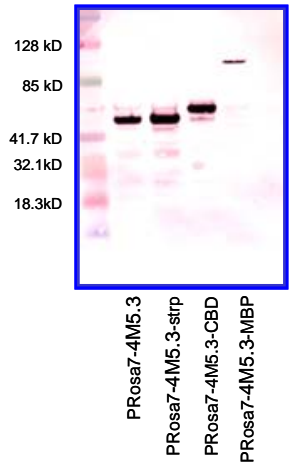

1)

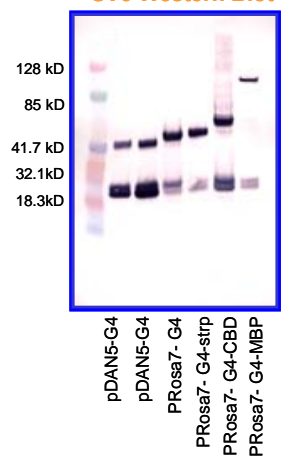

2)

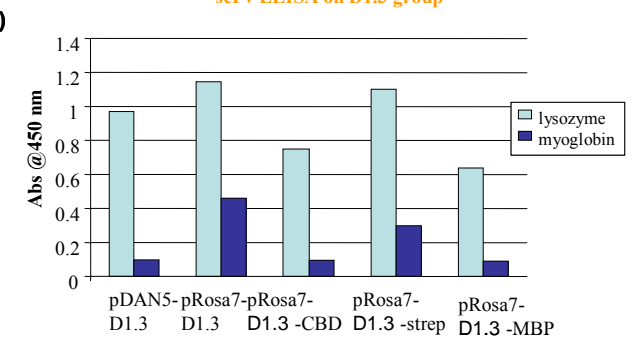

ScFv ELISA on D1.3 group

D1.3 D1.3 D1.3 -CBD D1.3 -strep D1.3 -MB

scFv ELISA on 4M5.3 group

2)

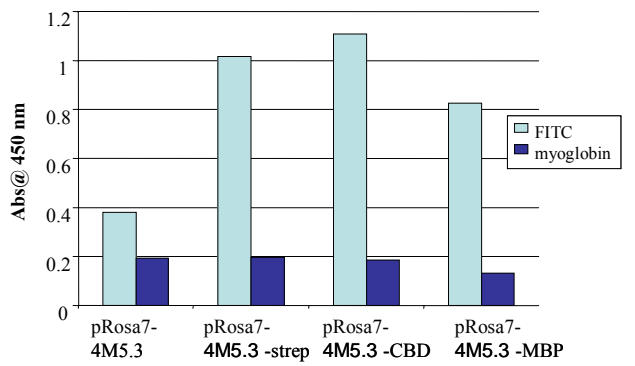

scFv ELISA on G4 Group

2)

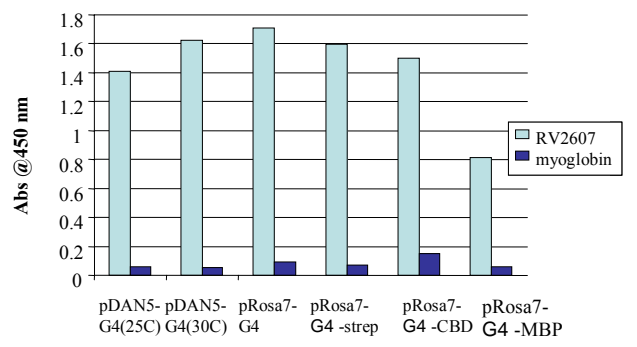

Figure 3-17. SV5 western and ELISA assay on the tagged single chains D1.3, 4M5.3 and G4 
Western Blot analysis demonstrated that all scFv-tag fusions produced protein of the expected size; and ELISA data confirmed that the protein interactions between D1.3, 4M5.3 and G4 and their respective antigens, lysozyme, fluorescein (FITC) and RV2607 was not affected by the presence of either strep tag II, CBD or MBP tag.

\subsubsection{Creation of pRosa8:}

PRosa 8 was created by removing gene 3 from pRosa7 (Refer to Figure 3-8). Gene 3 encodes g3p, a phage coat protein that is used for the display of proteins (antibodies) on the surface of bacteriophage. The gene was cloned into the expression tagged vectors (pRosa) with the thought of being exchanged by tags to be cloned downstream of the single chain. The expression of the gene 3 fused to a protein is known to be involved in bacterial toxicity. ${ }^{78}$. Gene3 is preceded by an Amber stop codon which should stop the translation of the gene using the appropriate cell strain (e.g.: BL21(DE3)). However as basal expression may occur, it was removed as its presence was not required for the purpose of the studies.

The pRosa7 vectors were digested with NotI, dropping the fragment of $1289 \mathrm{bp}$ corresponding to the gene. The bigger fragment, in each case, was re-ligated creating the pRosa8 plasmid. The resulting DNA was checked by restriction enzyme digestion. Each DNA clone was sequenced with T7prom and T7term primers and in the case of the plasmids with MBP, the MBP5 primer was also used to check that the DNA encoding gene3 was removed and all the vector features were in frame. To demonstrate that the removal of gene 3 protein did not compromise the antigen interaction, ELISA was performed in D1.3 pRosa8 clones. 


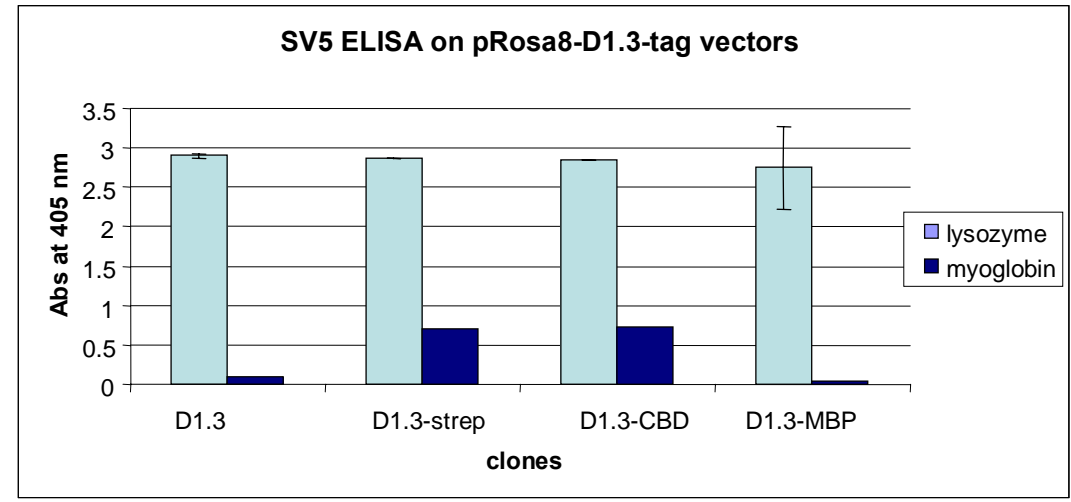

Figure 3-18. ELISA assay in D1.3-tag fusions expressed from pRosa8 vectors

ELISA results indicate that the removal of the gene didn't affect the antigen/antibody interaction.

\subsection{Functionality of the Tags:}

The functionality of each MBP, Strep-tag II and CBD tag was studied by interaction with appropriate bead matrices as G4-tag fusions (G4 is a scFv selected against TB protein RV2607, Refer to Chapter 4, section 4.2). Each G4-tag fusion was at the same time purified using Ni- NTA chromatography utilizing the C-terminus His tag as a control of purification.

The amount of each matrix to use (Chapter2: beads for affinity tags) was determined by the one with the highest binding capacity (Ni-NTA). $25 \mu 1$ of Ni-NTA matrix was used and the corresponding volume to work with the same capacity of the chitin beads, strep Tactin and amylose beads (Refer to Table 3-4). All the beads were in slurry of 50:50 resin: buffer. 


\section{Bead capacity}

\begin{tabular}{|c|c|c|c|}
\hline & & $\begin{array}{c}\text { beads for } \\
\sim 0.25 \mathrm{mg} \text { prot }\end{array}$ & slurry \\
\hline Ni- NTA beads & $\begin{array}{l}0.5-1 \mathrm{mg} \mathrm{prot} / 100 \mu \mathrm{l} \\
\text { resin }\end{array}$ & $25 \mu \mathrm{l}$ beads & $50 \mu \mathrm{l}$ \\
\hline Chitin & $2 \mathrm{mg}$ prot / $1 \mathrm{ml}$ bead & $125 \mu$ l beads & $250 \mu \mathrm{l}$ \\
\hline Amylose & $3 \mathrm{mg}$ prot $/ 1 \mathrm{ml}$ bead & $83.5 \mu \mathrm{l}$ beads & $167 \mu \mathrm{l}$ \\
\hline Strep-tactin & $3 \mathrm{mg}$ prot / $1 \mathrm{ml}$ bead & $83.5 \mu \mathrm{l}$ beads & $167 \mu \mathrm{l}$ \\
\hline
\end{tabular}

Table 3-4: bead capacity and amounts used for the experiments.

The protein produced by each G4-tag clone was expressed (Chapter 2: protein expression). The expressed protein, were recovered from the periplasmic extract, as described in chapter 2. Each construct contained either the strep tag II, MBP or CBD tag at the $\mathrm{N}$ terminus of the G4 scFv. Hexa-Histidine (His tag) and SV5 tags were also located at the $\mathrm{C}$ terminus of the $\mathrm{scFv}$ and are used routinely for purification and detection.

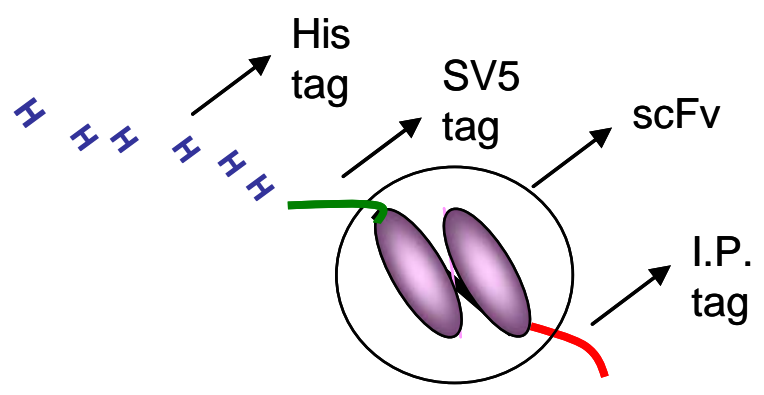

Figure 3-19. schematic of the expressed protein-tag fusions 
The tagged proteins were purified against their corresponding matrices, and in addition were analyzed against a non-specific matrix used as negative control of purification.

\begin{tabular}{|c|c|c|}
\hline scFv-tag & Beads & expected interaction \\
\hline \multirow{3}{*}{ G4-flag } & Ni-NTA & + \\
\cline { 2 - 3 } G4-Strep & Chitin & - \\
\cline { 2 - 3 } & Ni-NTA & + \\
\cline { 2 - 3 } & Strep-tactin & + \\
\hline \multirow{3}{*}{ G4-CBD } & Amylose & - \\
\cline { 2 - 3 } & Ni-NTA & + \\
\cline { 2 - 3 } & Chitin & + \\
\hline \multirow{3}{*}{ G4-MBP } & Amylose & - \\
\cline { 2 - 3 } & Ni-NTA & + \\
\cline { 2 - 3 } & Amylose & + \\
\hline
\end{tabular}

Table 3-5: Fusions of G4 to tags and beads used to compare purifications and expected interaction with the matrices.

Ni beads

specific beads

un-specific beads
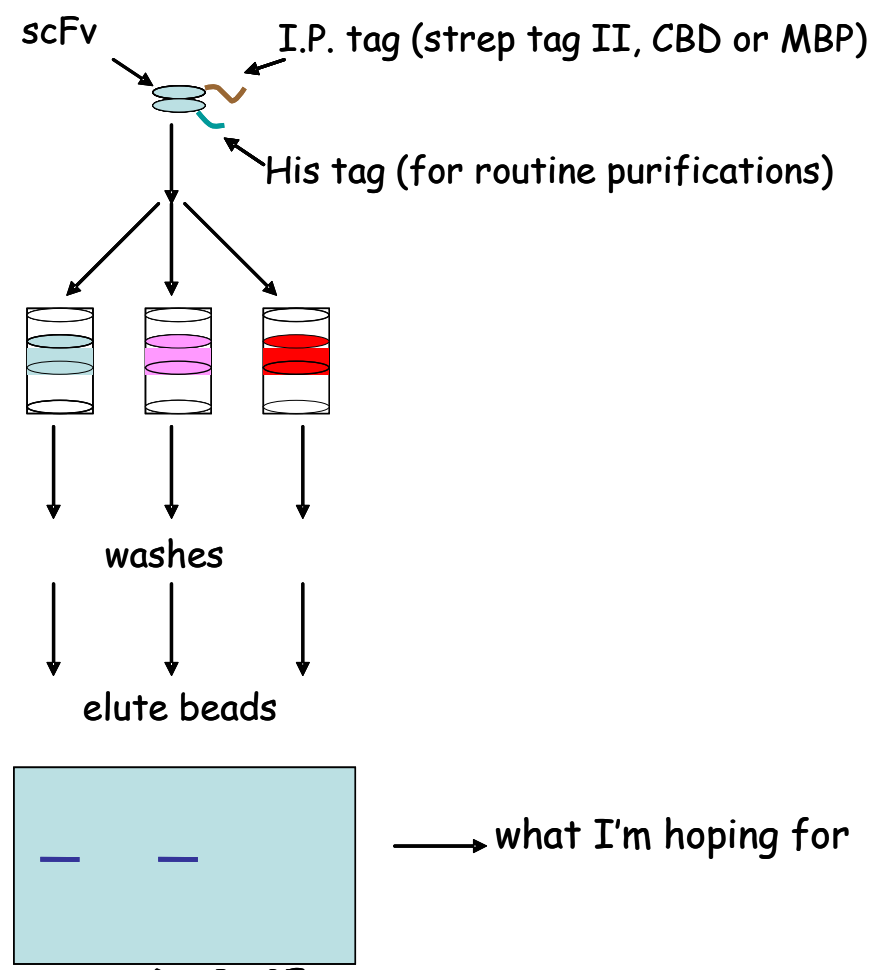

$\longrightarrow$ what I'm hoping for

SDS PAGE

Figure 3-20. Schematic of the experiment designed to check for the affinity and specificity of the tags

The retained protein was eluted from the matrix using specific elution buffer in each case except for the CBD tag (Chapter 2: Tag purification; buffers and protocols). 
The chitin binding domain could not be eluted from the chitin beads as the CBD protein lacks a cleavage site. In this case, the CBD fusion protein was removed from the beads by boiling 9

Three fractions of $700 \mu 1$ of periplasmic extract $\left(1 / 10^{\text {th }}\right.$ of the p.e. derived from $60 \mathrm{ml}$ of culture) from each G4-tag fusion, were used for independent purifications. G4tag fusions were first incubated with the washed beads and then placed into an empty column (BioRad) and allowed to settle by gravity, this first flow sample was kept for analysis (Refer to chapter 2: Tag purifications; buffers and protocols). Each column was washed with the specific washes designed for each type of beads. All the washes were collected, and $2 \mu 1$ of each spotted in two nitrocellulose membranes. The first membrane was stained using MemCode Reversible Protein Stain kit (Pierce). The second was developed as a Western Blot (Chapter2: SV5 western). This was done to determine if the washes were enough to remove un-bound material using $\alpha$-SV5 antibody to detect the presence of SV5 fused protein. The protein staining assay and the Western Blot determined that the washes were enough to remove the non-specific material. Each column (except the one containing the chitin matrix) was treated with the specific buffer (chapter 2: tag purification; buffers and protocols) to elute the captured proteins from the matrix. The eluted fractions were collected for analysis. Each sample produced in the assay (including initial, flow through, wash and elution) was analyzed by protein staining with Memcode and Western Blot analysis. The G4-CBD tag fusion protein was eluted from the column by boiling in $100 \mu \mathrm{l}$ of $4 \mathrm{x}$ loading dye at $100^{\circ} \mathrm{C}$ for $15 \mathrm{~min}, 15 \mu 1$ were used to run in gel. 
amylose beads
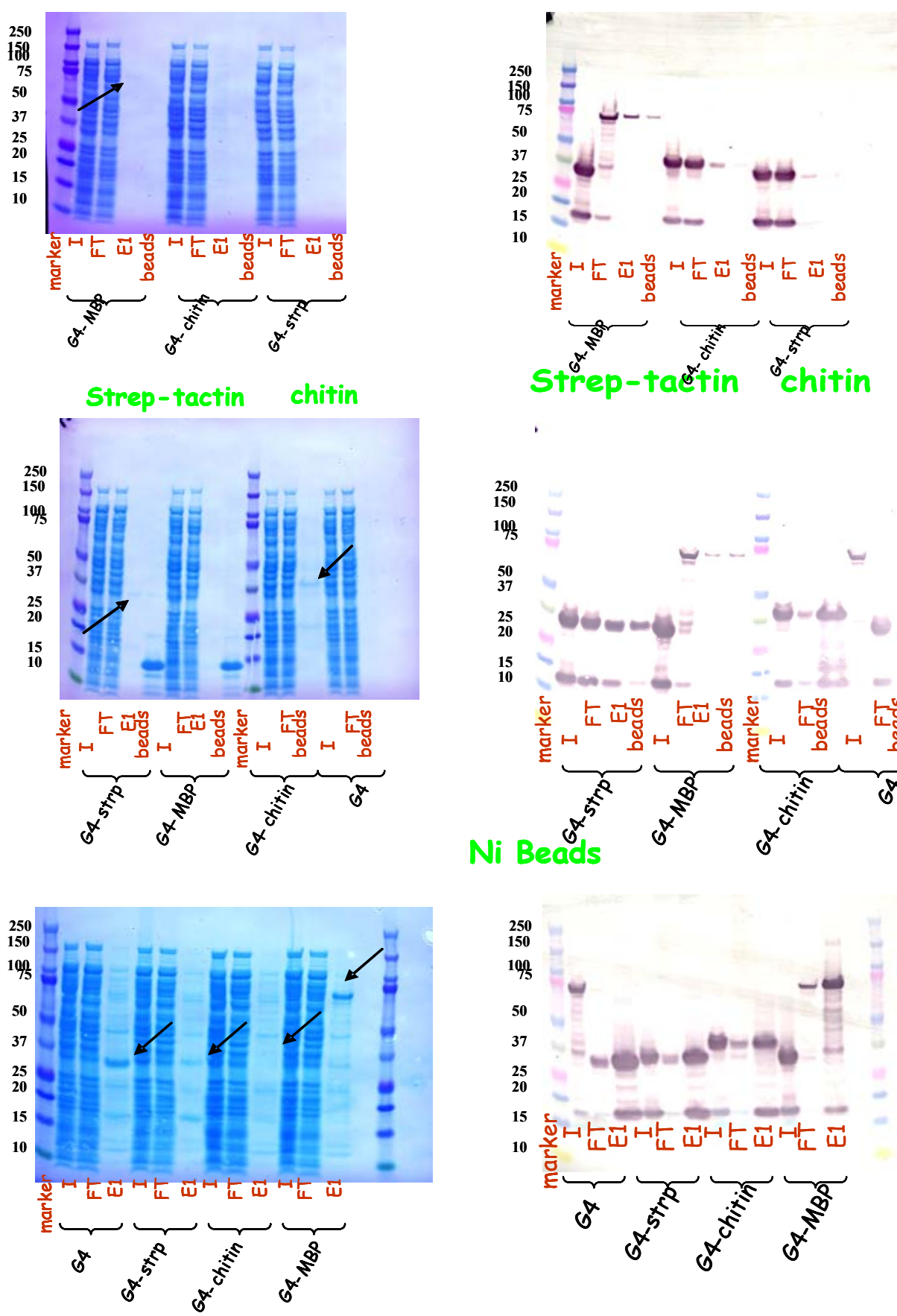

Figure 3-21. SDS-PAGE gel and SV5 western to prove the specificity of the affinity tags and the possibilities to use them as purification tags

I: initial sample; FT: flow through; E: elution; beads: boiled beads

* Initial samples of G4 and G4-MBP were swapped when loading in the gel 
The affinity purifications using the I.P. tags indicated that the tags bound specifically to the specific resin while most of the non-specific material was washed off. Although the hexa-Histidine tag purified more of the $\mathrm{scFv}$, there were also more contaminants present. All the I.P. tags had comparable yields but strep-Tactin gave a very clean product with washes at physiological conditions. A strong band of approximately $14 \mathrm{kD}$ was present in the fraction where the beads were boiled. This band corresponds to material released after boiling the strep Tactin beads, having not relation to the sample previously eluted. Chitin proved to be a high affinity matrix but with a very harsh elution protocol, due to the need of boiling to release the material. 


\subsection{Discussion}

This chapter was concerned with:

- Creation of expression vectors, pRosa, that allowed the simple cloning of single chains selected from pDAN5 libraries.

- Study of the influence of Immunoprecipitation tags, strep tag II, CBP, GST, $\mathrm{MBP}$ and $\mathrm{CBD}$, in the binding to the antigen when expressed fused to the single chains

- Use of the tags as purification handles.

- This chapter describes the creation of the multi-functional protein expression vector pRosa.

The vector was engineered to allow directional sub-cloning of DNA encoding single chain antibody proteins derived from pDAN5. In addition, the vector was designed to allow substitution of different purification or I.P. tags. The pRosa vector, derived from pET28, harbors the strong T7 promoter that enables the over-expression of the gene in T7 polymerase background (e.g. BL21 (DE3)) as well as two tags, hexa-Histidine and SV5. The vector was also designed with the possibility of $\mathrm{C}$ or $\mathrm{N}$ terminus cloning of the I.P. tags by substitution of the gene 3 or the flag tag. The tags for the experiments were cloned at the $\mathrm{N}$ terminus. $\mathrm{N}$ termini tags can facilitate the correct folding of the protein fused to them.

The pDAN5 vector is used for display and is the origin of the scFvs antibodies. The pDAN5 vector contains a hexa-Histidine and an SV5 tag at the C terminus of the scFv. The PDAN5 vector was modified for these studies by the introduction of NcoI, AgeI and SfiI R.S. and the gene encoding the flag tag at the $\mathrm{N}$ terminus of the scFv. The 
introduction of the flag tag was designed to allow easy substitution for any of the I.P. tags under study. The resulting vector was named pDAN6. PRosa6 was created by the introduction of the flag tag-scFv-His tag-SV5 tag encoding DNA fragment form pDAN6 into pET28.

The MBP, GST, CBD, Strep tag II and CBP tags were chosen for these studies. These have a wide range of molecular weight, going from very large (MBP, 43kD) to very small (strep tag II, $2.4 \mathrm{kD}$ ). While small tags should not interfere with the binding of the antigen to the antibody large ones may, however large ones might be of help in solubilizing and stabilizing the protein fused to them ${ }^{10}$. These tags also have high affinity for their correspondent matrices as well as diverse types of elutions, from mild to harsh, which might determine in which step to use them in a double tag purification process.

In some cases the affinity tag has been found to partially cover a biologically relevant binding site of the target protein, thus "masking" a functional ligand binding or cofactor binding site ${ }^{11}$. For that reason each tag while fused to the single chains needs to be checked not only for the right size of final protein and functionality of the tag, but also for no interference on the binding of the antibody to the antigen.

The size of the expressed protein-tag fusions were analyzed by Western Blot analysis using $\alpha$-SV5 antibody as secondary antibody. The clones containing the Strep tag II, CBP, and MBP tags produced expressed protein of the expected size. The GST fusion failed to express. Results show that I.P. fusion proteins that expressed, also bound their corresponding antigen when analyzed by ELISA, single chain antibody-I.P. tag fusions, produced higher ELISA signals compared to $\mathrm{scFv}$ proteins with no tag, 
concluding that the presence of most of the chosen tags was not detrimental to the binding to the antigen.

It is possible that the I.P. tag fusion aids protein solubility and/or folding. The signal obtained from the interaction with the antigen in the ELISA assay was better for the construct with the MBP tag followed by CBD, Strep tag II, construct with no tag and CBP. CBP and GST tags were discarded as the expression yield was not sufficient for these studies.

Further studies were carried out to determine if the tags had similar effect while fused to different single chains. The strategy for switching out the single chains included directional cloning by double digestion with BssHII and NheI enzymes. However, a second BssHII site present in the LacI gene of pRosa6 needed to be removed. The removal of the LacI gene BssHII site created pRosa7. The tags were added into this mutated vector by digestion from the pRosa6 vectors.

The three different single chains, D1.3, 4M5.3 and G4, were cloned into pRosa7 and expressed fusions with each I.P. tag. Expression of the panel of $\mathrm{scFv}$-tag fusion proteins was analyzed by Western Blot assay and ELISA. Results indicated that the tag fusions didn't hinder production of any of the single chain proteins and in some cases lead to an increase, (indicated by a stronger signal in the Western Blot). None of the tag-fusions impeded scFv activity when analyzed by ELISA.

PRosa 8 was created by deletion of gene3. Gene 3 is only needed for the display of the single chain on the surface of M13 bacteriophage and it was included in the vector to give it more versatility with the possibility of substitution with C terminus I.P. tags, but is was not necessary for the present studies. Gene 3 was deleted by simple digestion with 
NotI restriction enzyme. Expression of the scFv-tag panel in pRosa8 gave similar results to pRosa7 proving that gene 3 was not required.

It has been demonstrated that the functionality of the scFvs is not impeded by the I.P. tag fusions. In contrast, it was necessary to show that the I.P. tags were also functional when expressed as $\mathrm{scFv}$ fusions.

The aim of this analysis was not only to prove functionality of the tags but even more to determined which I.P. tag would perform best on the capture and purification of I.P. complexes. The requirements needed on the tag function included to enable protein purification in a simple process including mild wash and elution steps.

It was preferable to use a tag that required mild wash and elution conditions and therefore with a greater chance of recovering intact protein complexes. The idea was to try a double purification process where the first purification step could release intact complexes and achieve a cleaner product with a second purification step.

The $\mathrm{scFv}$ protein purification using the I.P. tags fused to them was compared to a standard purification method using the hexa-Histidine tag (located at the $\mathrm{C}$ terminus of the $\mathrm{scFv}$ ) (Refer to Figure 3-19). The scFv-tag fusions were extracted from the periplasm, captured by the specific beads of each tag, washed and eluted, using gravity flow. The proteins captured by the strep-tactin and amylose beads were eluted using specific buffers. In contrast, proteins captured by the chitin matrix were released by boiling (due to the lack of cleavage site in the CBD). All of the bead matrices captured and purified the specific tagged fusion proteins. There was no cross-reactivity between I.P. tags and non-specific matrices. 
In conclusion, the purification showed that MBP and strep tagII required mild washed and elutions in order to purify tagged scFv. Strep tag II required the mildest conditions. Chitin matrix was very effective for purifying CBD protein fusions. However, the use of CBD tags is limited to either the second stage of a double purification or a single purification, due to the harsh elution conditions that cause complete denaturation of the complex.

All the cloned tags, MBP, strep tagII, and CBD were proven to be functional when expressed as $\mathrm{scFv}$ fusions.

Strep tag II stands out as the tag with closer characteristics to the ideal tag for the purification of protein complexes (Refer to Chapter 1, Table 1-2). It is a very small tag, therefore no interference with the binding of the antigen to the antibody is expected. Purification is simple and provides clean product in a single step. It is done under physiological conditions, being able to keep the protein complex intact, therefore allowing for a second purification step. Based in all these reasons strep tagII was the tag of choice for purification of I.P. complexes in this thesis.

SV5 tag, although not tested in these previous experiments, will be the second tag chosen for protein complex purification in a double purification format (Refer to Chapter 5). SV5 is a very small tag (14 aminoacids) that interacts with high affinity with anti-SV5 antibody $^{1}$. It is cloned downstream of the scFv in the pDAN5 and pRosa vectors, opposite to the I.P. tags. Purification using two tags, each at opposite site of the scFv, will assure the purification of complexes that interact only with full length scFvs. Its presence doesn't interfere with the antibody function and it is usually used as detection tag in Western blots and ELISA. Elution is done in harsh conditions with high salt and low $\mathrm{pH}$ 
buffers which disrupt the complex, for this reason it will be used as a second step in a double purification process.

\subsection{Abbreviations:}

R.E: restriction enzyme

CBP: calmodulin binding protein

CBD: chitin binding domain

MBP: maltose binding protein

scFv: Single chain variable fragment

kbp: kilo base pairs

TB: tuberculosis

ELISA: Enzyme-Linked ImmunoSorbant Assay

HRP:Horseradishperoxidase. 


\subsection{References}

${ }^{1}$ Sblattero, D.; Bradbury, A., Exploiting recombination in single bacteria to make large phage antibody libraries. Nat Biotechnol 2000, 18, (1), 75-80.

${ }^{2}$ Studier, F. W.; Moffatt, B. A., Use of bacteriophage T7 RNA polymerase to direct selective high-level expression of cloned genes. J Mol Biol 1986, 189, (1), 113-30.

${ }^{3}$ Velappan, N.; Martinez, J. S.; Valero, R.; Chasteen, L.; Ponce, L.; BonduHawkins, V.; Kelly, C.; Pavlik, P.; Hjelle, B.; Bradbury, A. R., Selection and characterization of ScFv antibodies against the Sin Nombre hantavirus nucleocapsid protein. J Immunol Methods 2007, 321, (1-2), 60-9.

${ }^{4}$ Siegel, R. W.; Velappan, N.; Pavlik, P.; Chasteen, L.; Bradbury, A., Recombinatorial cloning using heterologous lox sites. Genome Res 2004, 14, (6), 1119-29.

${ }^{5}$ Mariuzza, R. A.; Jankovic, D. L.; Boulot, G.; Amit, A. G.; Saludjian, P.; Le Guern, A.; Mazie, J. C.; Poljak, R. J., Preliminary crystallographic study of the complex between the Fab fragment of a monoclonal anti-lysozyme antibody and its antigen. J Mol Biol 1983, 170, (4), 1055-8.

${ }^{6}$ Boder, E. T.; Midelfort, K. S.; Wittrup, K. D., Directed evolution of antibody fragments with monovalent femtomolar antigen-binding affinity. Proc Natl Acad Sci U S A 2000, 97, (20), 10701-5.

${ }^{7}$ Chasteen, L.; Ayriss, J.; Pavlik, P.; Bradbury, A. R., Eliminating helper phage from phage display. Nucleic Acids Res 2006, 34, (21), e145.

${ }^{8}$ Rampf, B.; Bross, P.; Vocke, T.; Rasched, I., Release of periplasmic proteins induced in $\mathrm{E}$. coli by expression of an $\mathrm{N}$-terminal proximal segment of the phage fd gene 3 protein. FEBS Lett 1991, 280, (1), 27-31.

${ }^{9}$ Arakane, Y.; Zhu, Q.; Matsumiya, M.; Muthukrishnan, S.; Kramer, K. J., Properties of catalytic, linker and chitin-binding domains of insect chitinase. Insect Biochem Mol Biol 2003, 33, (6), 631-48.

${ }^{10}$ Hanke, T.; Randall, R. E., Variable domain sequences of mAb with high affinity for a linear oligopeptide. Immunogenetics 1995, 42, (5), 442-3.

${ }^{11}$ Casey, J. L.; Keep, P. A.; Chester, K. A.; Robson, L.; Hawkins, R. E.; Begent, R. H., Purification of bacterially expressed single chain Fv antibodies for clinical applications using metal chelate chromatography. J Immunol Methods 1995, 179, (1), 105-16. 
${ }^{1}$ Randall, R. E.; Young, D. F.; Goswami, K. K.; Russell, W. C., Isolation and characterization of monoclonal antibodies to simian virus 5 and their use in revealing antigenic differences between human, canine and simian isolates. $J$ Gen Virol 1987, 68 ( Pt 11), 2769-80. 


\section{Chapter 4. Selection and Analysis of scFvs}

\subsection{Introduction:}

Several selections of scFvs antibodies against different targets were carried out to obtain multiple single chains for these studies. Two different types of selection were used.

- Immunotube selection, in which the target antigen is bound to a plastic surface

- In solution selection, in which the biotinylated target antigen is captured by streptavidin-coated magnetic beads.

Immunotube selection against tuberculosis TB antigen RV2607 produced a scFv, G4. This scFv was used to study the influence of the tags (Refer to chapter 3, section 3.6.1). Other selections were performed "in solution" against five additional Shewanella proteins (SO0256, SO0316, SO2603, SO0090, SO1268). Multiple single chains were obtained by this manner and the specificity of the antibody for its corresponding antigen was checked using flow cytometry and ELISA assays. Singles chain antibodies selected against two of the five Shewanella antigens were cloned into pRosa8-strep vector for further immunoprecipitation experiments. All the selections were done using a large phage antibody library ${ }^{1}$. 


\subsection{Immunotube selection against RV2607:}

ScFv antibodies were selected against TB RV2607 antigen in immunotubes as described in chapter 2: immunotube selections. During immunotube selection, the antigen is coated over the immunotube surface and the library is incubated inside the tube. Wash and elution processes are carried out within the immunotube to recover the scFvs that bind specifically to the target antigen (Refer to Figure 4-1).

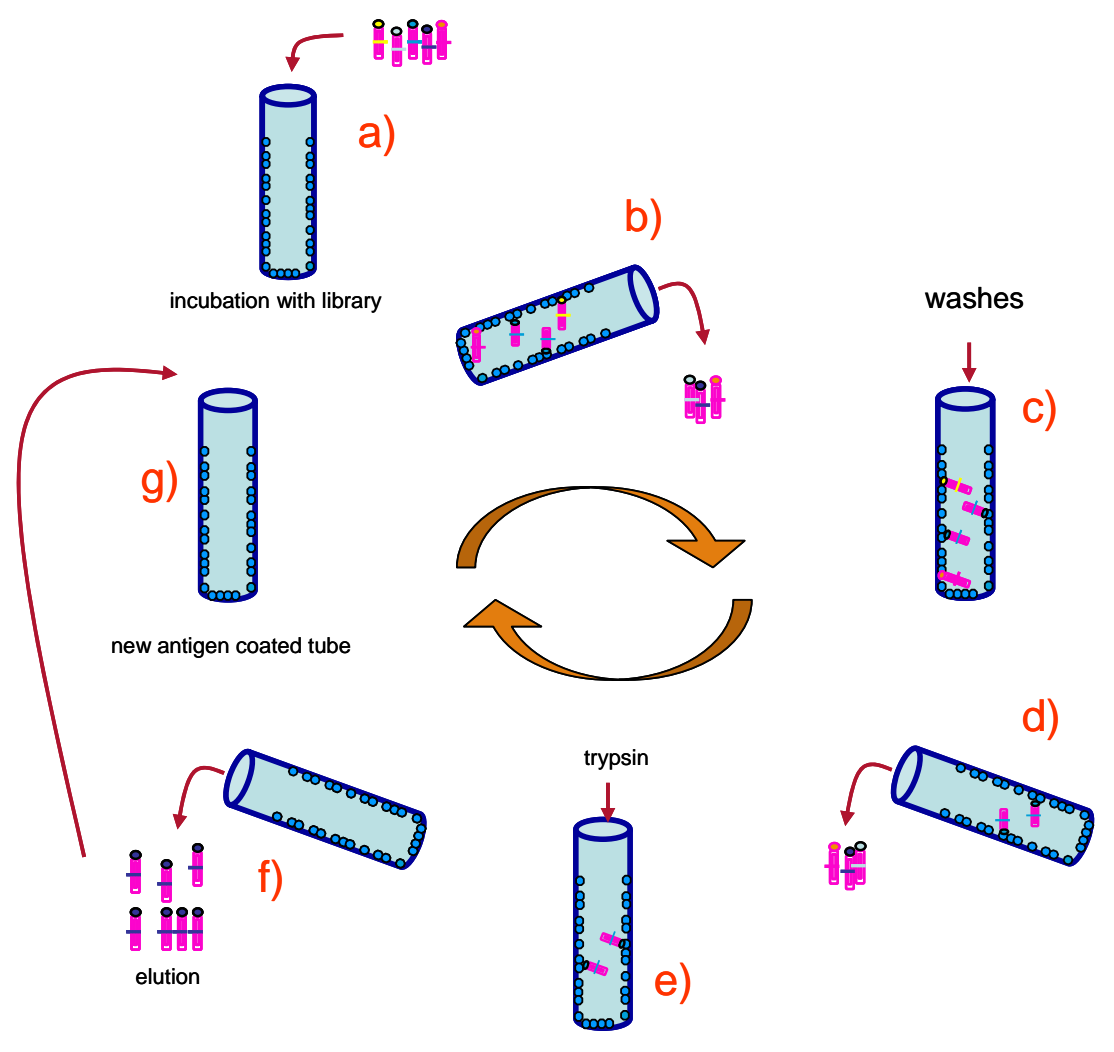

Figure 4-1. Schematic of immunotube selection: a)Incubation of phage-scFv library with antigen coated immunotube, b) Removal of unbound phages, c) Wash in immunotube to remove non-specific binders, d) Removal of wash buffer. 
Following three rounds of selection, 94 clones were isolated for further screening to test the binding activity of the recovered scFvs against the target antigen and alcohol dehydrogenase (negative control), by scFv ELISA. Binding events were reported using horseradish peroxidase (HRP) conjugated to anti-mouse immunoglobulin. Positive clones were identified as those producing an HRP signal at least 3 fold higher than the negative control (data not shown). $\mathrm{PCR}$ amplification with $\mathrm{pDpH}$ ' and $\mathrm{pDpH} 3$ ' primers (flanking antibody gene) determined which of the selected clones contained full length scFvs (containing both, VH and VL genes). The clones, A7, G4, G12, gave PCR product of the expected size (approximately $800 \mathrm{bp}$ ) whereas clone B2 gave a PCR product of smaller size indicating VL or VH deletions. (Refer to Figure 4-2). The full length clones, A7, G4 and G12 were fingerprinted with BstNI restriction enzyme. Results show that A7 and G4 are the same scFv clone while G12 has a different pattern, corresponding to a different single chain (Refer toFigure 4-3)

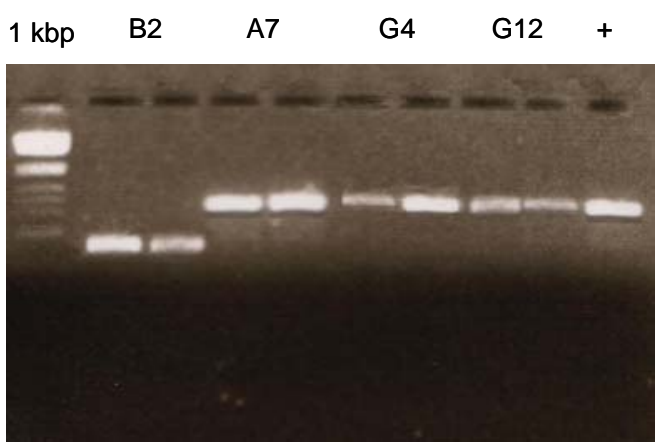

Figure 4-2. pDpH5'/pDpH3' amplification of ELISA positives 

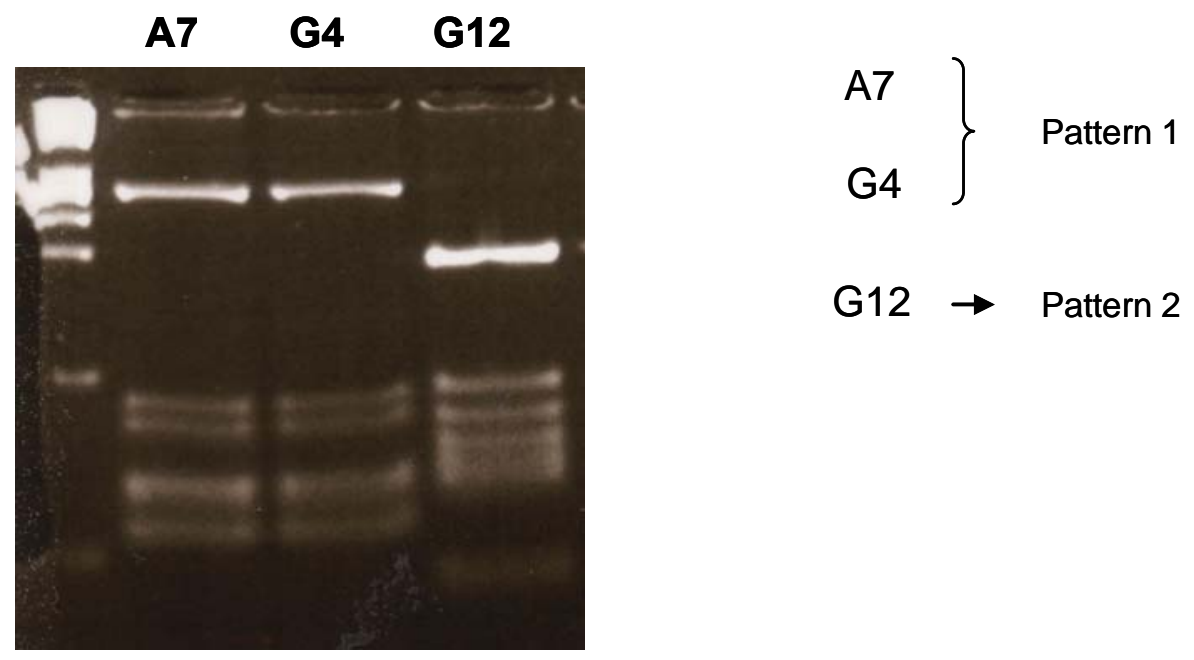

Figure 4-3. BsTN1 R.E. digestion of the full length single chains. 2 different fingerprints were identified.

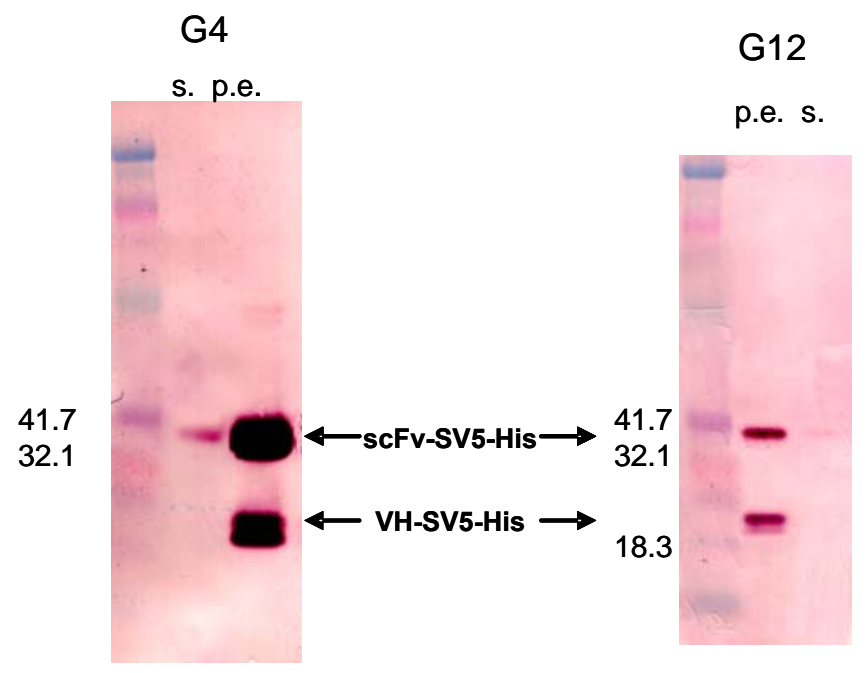

Figure 4-4. : SV5 westerns on clones G4 and G12 (marker: kaleidoscope Prestained Standard (BIORAD))

Each of the two different scFv clones, G4, and G12, were grown in $50 \mathrm{ml}$ of 2xTy media and induced with IPTG (Chapter 2: protein expression). The expressed protein was recovered from the cell periplasm and both, the media supernatant (s) and the periplasmic 
extract (p.e.); (Chapter 2: periplasmic extraction) were analyzed for the presence of $\mathrm{scFv}$ protein by SV5 Western blot (chapter 2: Western blot) (Refer to Figure4-4).

Both scFvs, G4 and G12 produced protein of the expected size, $35 \mathrm{kD}$, within the periplasmic extract. The $\mathrm{G} 4 \mathrm{scFv}$ was chosen as a candidate, because it exhibited higher expression levels and consistently good antigen recognition, to demonstrate that the scFvs remained functional when expressed as tag fusions (Refer to chapter 3, section 3.5).

\subsection{Selection against Shewanella antigens "in solution"}

In contrast to the immunotube selection previously described (chapter 4, section 4.2), $\mathrm{scFv}$ antibodies were selected against target antigens "in solution". ScFv antibodies were selected against five Shewanella antigens in this manner. Antigens were 1) biotinylated, 2) allowed to interact with library phages, 3) incubated with neutravidin coated magnetic beads (Dynabeads, Dynal). (Refer to chapter 2: Selection of antibodies using neutravidin magnetic beads). The bead-antigen-scFv complex is formed in solution and moved to different wells of the selection plate by the use of magnets. (Refer to section 4.6) where it is washed and eluted from the beads. 


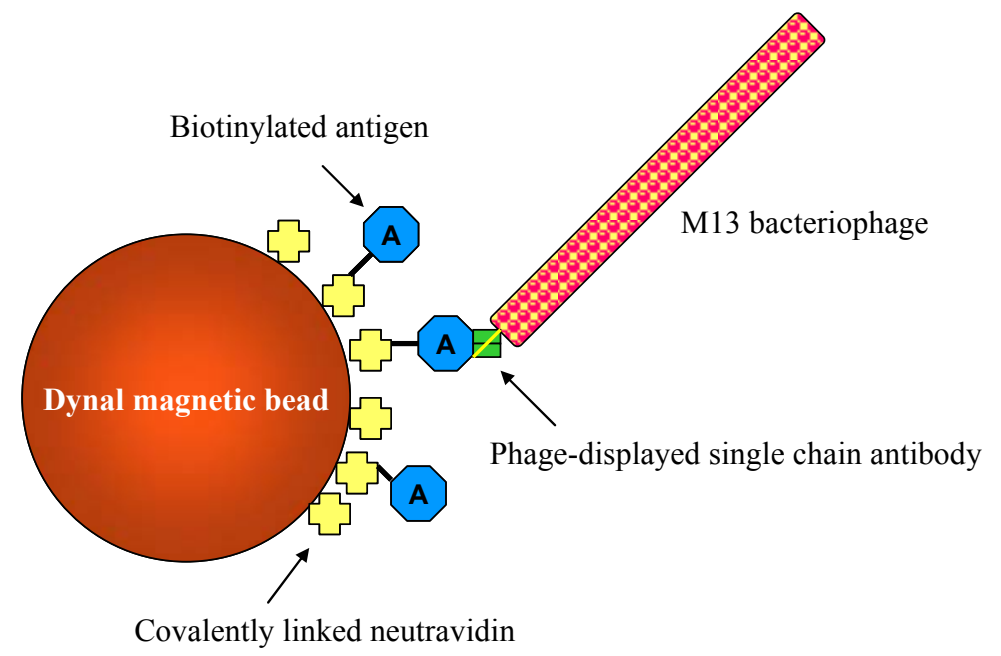

Figure 4-5. Schematic of interaction of antibodies with biotinylated antigens bound to neutravidin beads

\subsection{Biotinylation:}

Each target antigen was biotinylated using the EZ-link Sulfo-NHS-LC-LC-biotin kit (Pierce). The protocol was designed for protein concentrations of at least $2 \mathrm{mg} / \mathrm{ml}$ and suggested an increase of the biotin linker, in the case of more diluted samples, but with no guidance on amounts. The five target antigens were provided at concentrations more diluted than those recommended (as shown in to Table 4-1).

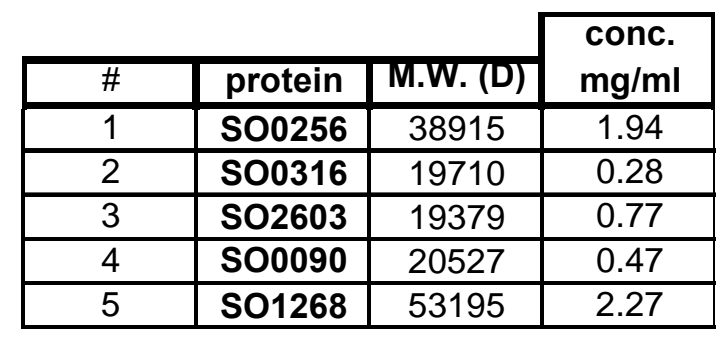

Table 4-1. Shewanella proteins information (concentrations before dialysis)

The conditions for biotinylation were determined as follows. The protocol suggested using at least a 20x excess of biotin linker for concentrations of $2 \mathrm{mg} / \mathrm{ml}$, 
however it was decided to increase the amount linearly. The lowest $(0.28 \mathrm{mg} / \mathrm{ml})$ and the highest $(2.27 \mathrm{mg} / \mathrm{ml})$ protein concentrations (before dialysis) were assigned an excess of 60x and 30x of biotin linker respectively (Refer to Table 4-2). An equation was defined and amount of linker excess, determined for each case. Biotinylation was carried out according to the manufacturer's instructions.

\begin{tabular}{|c|c|c|}
\multicolumn{2}{c|}{} & $\begin{array}{c}\text { times } \\
\text { linker excess }\end{array}$ \\
\hline 1 & protein & linker \\
\hline 1 & SO0256 & 35 \\
\hline 2 & SO0316 & 58 \\
\hline 3 & SO2603 & 51 \\
\hline 4 & SO0090 & 55 \\
\hline 5 & SO1268 & 30 \\
\hline
\end{tabular}

Table 4-2. Excess of biotin linker used for each protein

The presence of biotinylated antigen was analyzed via Western blot with streptavidin conjugated to alkaline phosphatase reporter (as shown in Figure 4-6). Results show that each protein was biotinylated and gave signal of expected molecular size.

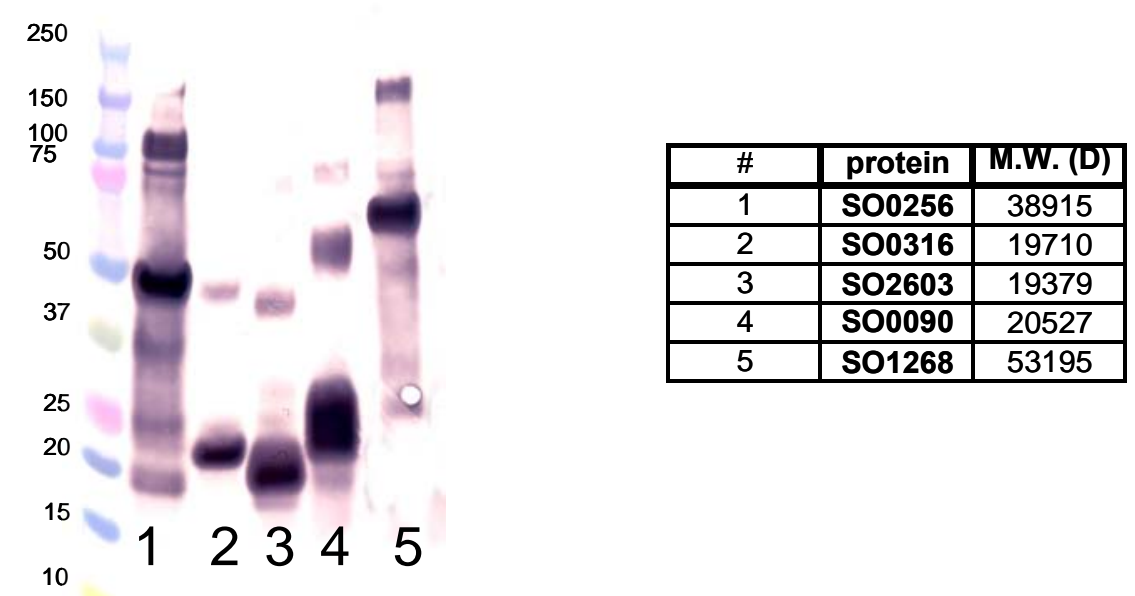

Figure 4-6. Western blot analysis of the biotinylated antigens. Loaded approximately 200ng of each protein. 
The concentration of each biotinylated protein was quantified using two methods. The total protein within each sample was analyzed using BCA Protein assay kit(Pierce). In contrast, the concentration of the purified protein was analyzed by SDS-PAGE to 1) Check for purity and the presence of proteolysis

2) Determine the concentration by colorimetry (comparing the protein band intensity against known BSA standards). Two SDS PAGE gels were used to analyze antigens with numbers 1, 3, 4, 5 against BSA standards (samples 2-5). Protein 2 was not analyzed using this method. 

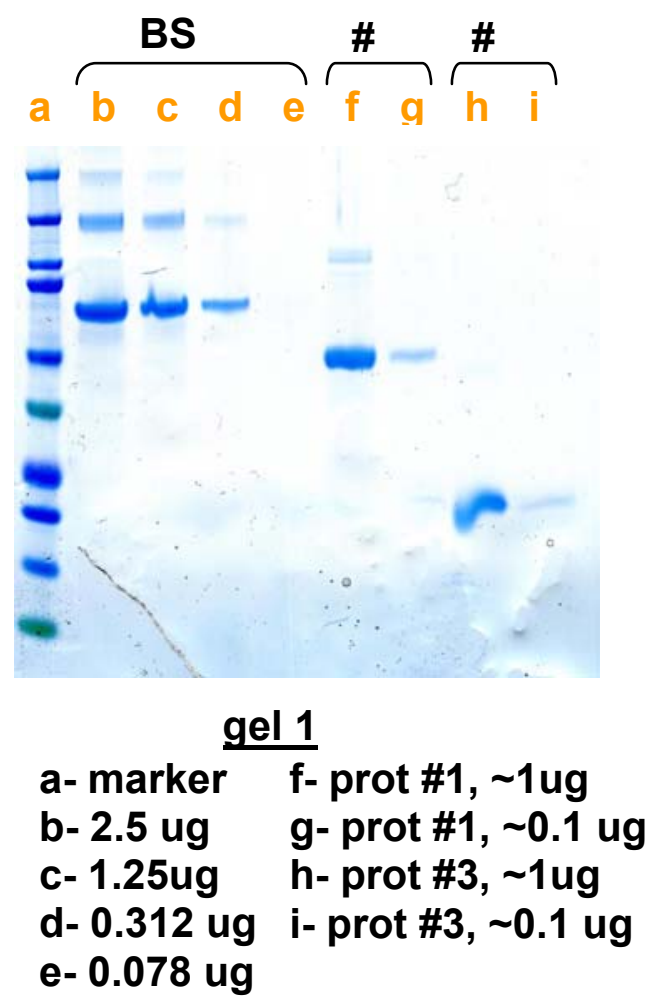
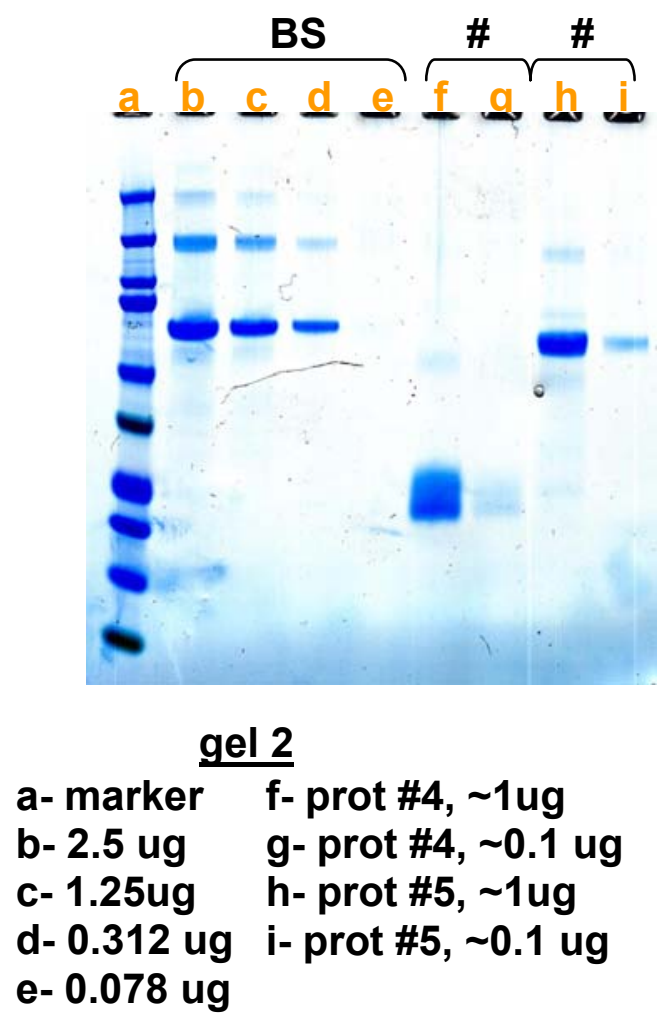

Figure 4-7. SDS- PAGE analysis for protein quantification by colorimetry

The amounts of protein loaded were based on those values produced using the BCA assay kit.

\begin{tabular}{|c|c|c|}
\hline prot\# & ug/ml & ug/ul \\
\hline $\mathbf{1}$ & 1020 & 1.02 \\
\hline $\mathbf{2}$ & 230 & 0.23 \\
\hline $\mathbf{3}$ & 230 & 0.23 \\
\hline $\mathbf{4}$ & 224 & 0.224 \\
\hline $\mathbf{5}$ & 720 & 0.72 \\
\hline
\end{tabular}

Table 4-3. Antigen concentrations calculated by BCA assay.

SDS-PAGE results demonstrate that the proteins remain intact and that the concentration of each protein corresponds to that calculated using the BCA kit. The concentration of the 
antigens must be known in order to calculate the level of Biotinylation. In addition, it allows for control of the amount of antigen used in each selection.

The level of protein Biotinylation was calculated for each antigen using the EZ Biotin Quantification Kit (Pierce) (Refer toTable 4-4).

\begin{tabular}{|c|c|}
\hline protein \# & biotin/ prot. molec. \\
\hline $\mathbf{1}$ & 2.3 \\
\hline $\mathbf{2}$ & 6.3 \\
\hline $\mathbf{3}$ & 21.5 \\
\hline $\mathbf{4}$ & 20.6 \\
\hline $\mathbf{5}$ & 0.7 \\
\hline
\end{tabular}

Table 4-4. Number of biotins per protein molecule for each of the antigens

The ideal biotinylation level was 1 biotin per protein molecule. However the levels ranged from 0.7 to 21 . The study was continued despite having "non-ideal" biotinylation levels in some cases, because of the limited amount of antigen available. 


\subsection{High throughput selection of scFvs}

Selections against the five antigens were carried out simultaneously using KingFisher $^{\circledR}$ (Thermo-Labsystems). Plastic covered magnetic rods allow the transfer of magnetic beads from well to well (Refer to Figure 4-8).

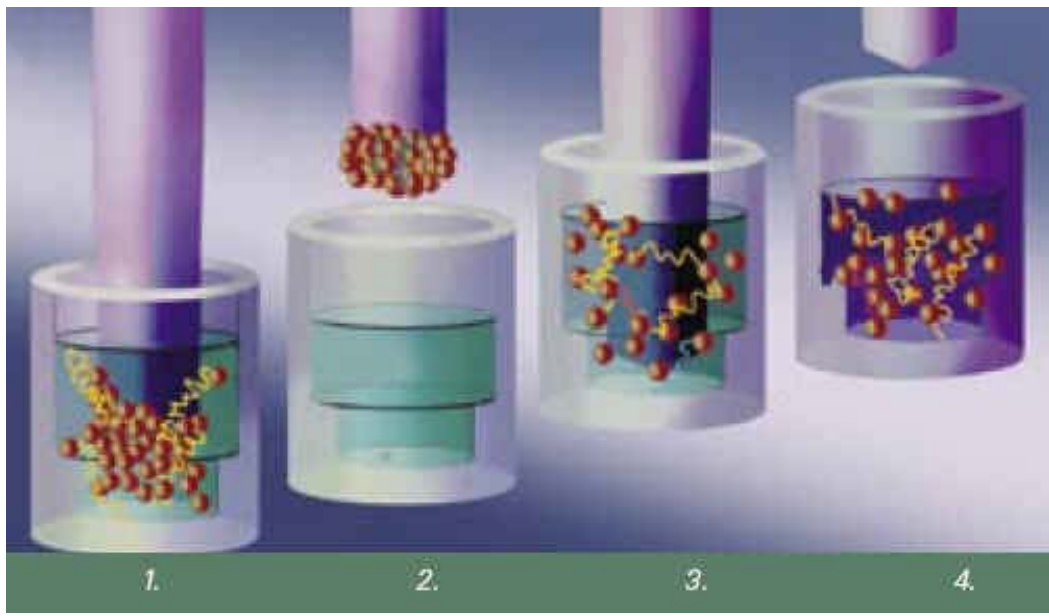

Binding Moving Washing Incubating

Figure 4-8. Schematic of the KingFisher ${ }^{\circledR}$ working principle. (From http://www.thermo.com)

Selection is performed in KingFisher ${ }^{\circledR}$ plates. (Refer to Figure 4-9). During the first step, library phages are incubated with biotinylated antigen, and then the complex is captured upon the neutravidin coated magnetic beads. The phages interacting with the antigen are transferred from well to well, during several washing steps; only phage displaying $\mathrm{scFv}$ that specifically binds to the target antigen is recovered following acid mediated elution. The eluted phage is quickly neutralized by addition of Tris solution and recovered by infection into host bacterial cells, DH5 $\alpha \mathrm{F}$. The infected cells containing the $\mathrm{scFv}$ binding population are grown overnight on agar plates with selective media. The 
number of bacterial colonies produced, gives and indication of the numbers of $\mathrm{scFv}$ binders recovered from the selection.

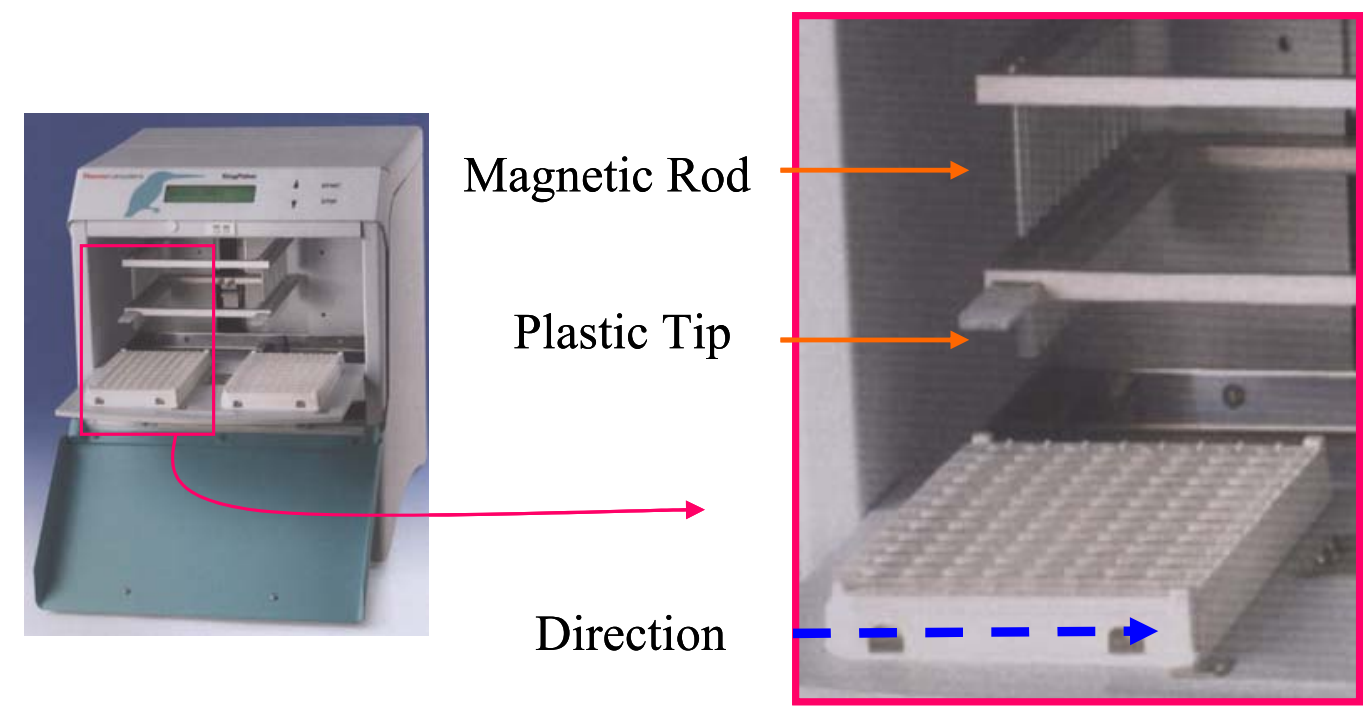

\begin{tabular}{|c|c|c|c|c|c|c|}
\cline { 2 - 6 } \multicolumn{1}{c|}{} & prot\# 1 & prot\# 2 & prot\# 3 & prot\# 4 & prot\# 5 & vol \\
\hline A & $\begin{array}{c}\text { antigen } \\
+\phi\end{array}$ & $\begin{array}{c}\text { antigen } \\
+\phi\end{array}$ & $\begin{array}{c}\text { antigen } \\
+\phi\end{array}$ & $\begin{array}{c}\text { antigen } \\
+\phi\end{array}$ & $\begin{array}{c}\text { antigen } \\
+\phi\end{array}$ & $\begin{array}{c}171 \mu \mathrm{ll} \text { lib }+19 \mu \mathrm{l} \text { antigen } \\
\text { incubate 1hr RT }\end{array}$ \\
\hline B & strep. beads & strep. beads & strep. beads & strep. beads & strep. beads & $200 \mu \mathrm{l}$ \\
\hline C & PBS-T & PBS-T & PBS-T & PBS-T & PBS-T & $190 \mu \mathrm{l}$ \\
\hline D & PBS-T & PBS-T & PBS-T & PBS-T & PBS-T & $190 \mu \mathrm{l}$ \\
\hline E & PBS-LT & PBS-LT & PBS-LT & PBS-LT & PBS-LT & $190 \mu \mathrm{l}$ \\
\hline F & PBS-LT & PBS-LT & PBS-LT & PBS-LT & PBS-LT & $190 \mu \mathrm{l}$ \\
\hline G & PBS-LT & PBS-LT & PBS-LT & PBS-LT & PBS-LT & $190 \mu \mathrm{l}$ \\
\hline H & $0.1 \mathrm{M} \mathrm{HCl}$ & $0.1 \mathrm{M}$ HCl & $0.1 \mathrm{M} \mathrm{HCl}$ & $0.1 \mathrm{M}$ HCl & $0.1 \mathrm{M}$ HCl & $150 \mu \mathrm{l}$ \\
\hline
\end{tabular}

${ }^{*}$ strep. beads: streptavidin coated beads.

Figure 4-9 Schematic of the KingFisher ${ }^{\circledR}$ instrument and plate set up for the selection of scFvs

Three rounds of selection were carried out and the output from each round was input for the following to allow enrichment of the binding population. In order to increase stringency of the selection conditions, the antigen concentration was decreased and the length of the washing steps increased in each subsequent round (Refer to Table 4-5) 


\begin{tabular}{|c|c|c|c|}
\cline { 2 - 4 } \multicolumn{1}{c|}{} & 1st round & 2nd round & 3rd round \\
\hline antigen concentration & $600 \mathrm{nM}$ & $60 \mathrm{nM}$ & $60 \mathrm{nM}$ \\
\hline washes & $1 \mathrm{~min}$ & $5 \mathrm{~min}$ & $15 \mathrm{~min}$ \\
\hline
\end{tabular}

Table 4-5. Variation in concentration and washes between the three rounds of selection

In the third round, a negative control was included to determine the level of $\mathrm{scFv}$ background binding (e.g. scFv that binds to streptavidin on the beads). The output from the second round was left to interact with beads in the absence of target antigen (negative control). In order to determine that scFv-phage with selective binding properties have been recovered; the ratio of scFv-phage that binds antigen is compared to the negative control. (Refer to Table 4-6).

\begin{tabular}{|c|c|}
\cline { 2 - 2 } \multicolumn{1}{c|}{} & $\begin{array}{c}\text { 3rd Round Results } \\
\text { \# colonies } \\
\text { antigen/negative }\end{array}$ \\
\hline Protein \# & $25 x$ \\
\hline 1 & $2 x$ \\
\hline 2 & $50 x$ \\
\hline 3 & $3 x$ \\
\hline 4 & $100 x$ \\
\hline 5 & \\
\hline
\end{tabular}

Table 4-6. Ratio between numbers of colonies obtained from third round output over antigen coated beads versus the one over empty beads (negative)

Results indicate recovery of those scFv-phages that specifically bind to the target antigen in all five cases. After the third round of selection, the bacteria infected with the output phages was recovered and stocked. 


\subsection{High-throughput screening of scFv}

Single chains produced in the third round selection output were tested for binding activity against the target antigen. The use of multiplex flow cytometry ${ }^{2}$, allows the screening of the binding activities of an individual scFv-phage against multiple targets in one analytical sample. These multiple targets include 1) The target antigen, against which the $\mathrm{scFv}$ was selected, 2) An antibody that recognizes the SV5 tag (that all the scFv are fused to and 3) Other antigens which the $\mathrm{scFv}$ is not expected to cross react. This allows comprehensive analysis of each individual $\mathrm{scFv}$ regarding expression levels, binding specificity and cross-reactivity of each clone in a simple measurement.

The multiplex screening assay utilizes Luminex carboxylated beads. The beads are intrinsically color-coded where each has a slightly different amount of either two dyes. Up to 100 different colored beads are available for multiplexing.

Different bead sets are distinguishable when excited with a red laser. $\mathrm{ScFv}$ antibodies are fluorescently labeled and the fluorescence obtained after excitation with green laser provided binding information of the single chains to the different bead sets.(Refer to Figure 4-10).

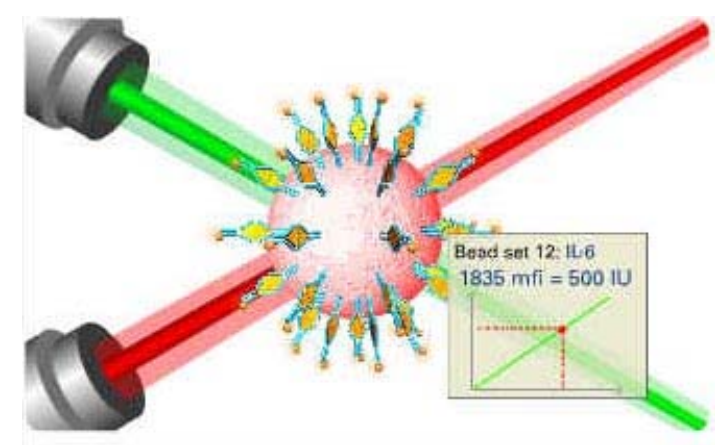

Figure 4-10:: Excitation of a Luminex bead with green and red lasers (Luminexcorp.com). 
Each $\mathrm{scFv}$ antigen produced from the selections, was screened against the four Shewanella antigens in multiplex, using the flow cytometry method described ${ }^{2}$. Each bead set was checked for uniformity in neutravidin coating, using MAG protein (monomeric azami green) as a standard (data not shown).

Each individual set of neutravidin coated beads was incubated with a different biotinylated antigen (antigens $\# 2,3,4,5$ ) and the four different bead sets were coupled as shown in Table 4-7 and Figure 4-18A.

\begin{tabular}{|c|c|}
\hline Bead Set & B-antigen \\
\hline Neutravidin xMAP 129 & b- $\alpha$-SV5 \\
\hline Neutravidin xMAP 142 & b-antigen \\
\hline Neutravidin xMAP 135 & b-ubiquitin \\
\hline Neutravidin xMAP 180 & PBS \\
\hline
\end{tabular}

Table 4-7: Antigens and microspheres used in flow analysis. (b- Stands for biotinylated) xMAP 142 bead set was coupled to the corresponding antigen \#2, 3, 4 or 5 according to the scFv output being screened. xMAP 129 was coupled to $\alpha$-SV5 antibody to indicate the levels of scFv protein expression. xMAP 135 was coupled to ubiquitin as a negative control and xMAP 180 was included to check that the scFv clones did not cross react with neutravidin.

\subsubsection{Labeling of the single chains ( $K$ coil-Alexa 488):}

The single chain antibodies need to be labeled with a fluorescent dye in order to be analyzed by flow cytometry. This was achieved by the use of coiled coils, a bundle of $\alpha$ helices that are wound into a superhelix ${ }^{345}$ (Refer to Figure 4-11). 


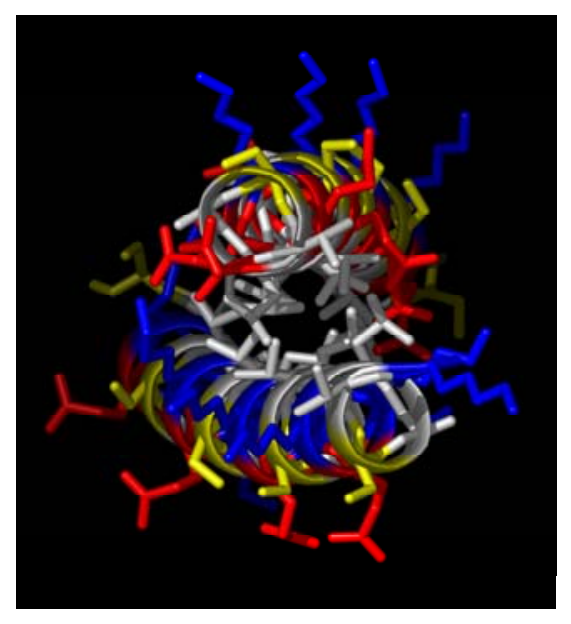

Figure 4-11: Coiled coils

Single chains are fluorescently labeled using synthetic $\mathrm{E} / \mathrm{K}$ coil heterodimeric peptides that bind with an affinity of approximately $60 \mathrm{pM}$. Single chains are expressed as E coil fusions and are mixed with $\mathrm{K}$ coil conjugated with Alexa 488 . The one to one $\mathrm{E} / \mathrm{K}$ coil interaction ensures uniform labeling of each scFv protein.

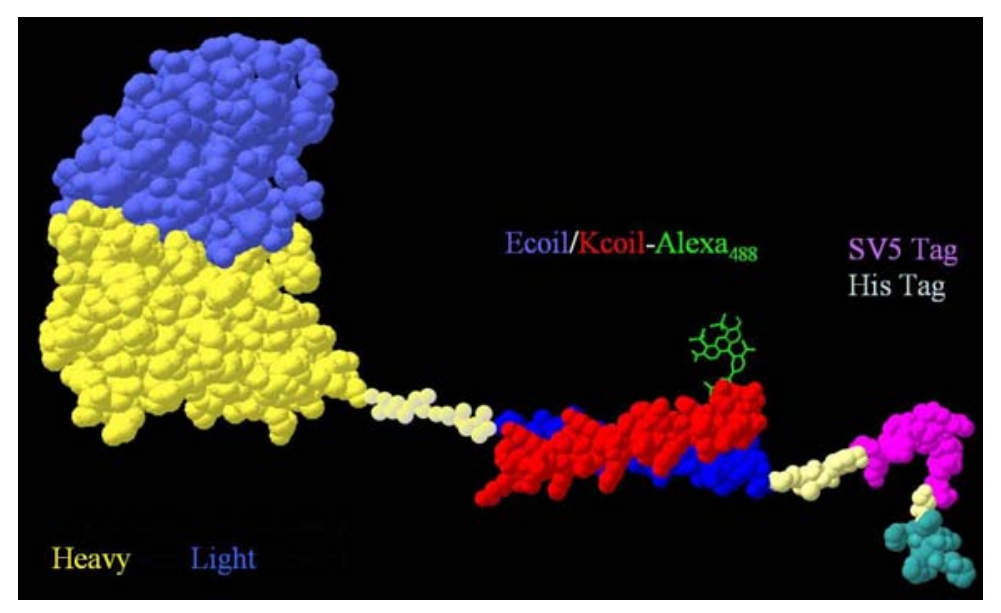

Figure 4-12: Coil mediated labeling of single chain.

E coil sequence: NH2-EVSALEKEVSALEKEVSALEKEVSALEKEVSALEK-OH

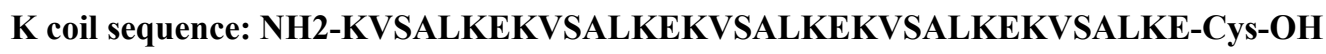


K coil was in-house synthesized in collaboration with Dr. J. Schmidt (LANL) using solid-phase chemistry and labeled at a Cys residue using sulfhydryl-reactive Alexa Fluor 488 maleimide reagent (Molecular Probes) (Chapter 2: K coil-Alexa 488 synthesis). Analysis of the synthesized peptide before and after labeling was done by mass spectrometry.

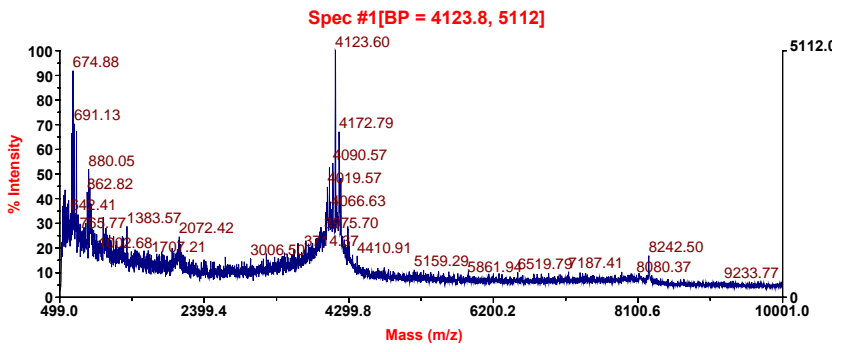

\begin{tabular}{|l|l|}
\cline { 2 - 2 } \multicolumn{1}{c|}{} & M.W. \\
\hline K coli+ Fmoc & 4,122 \\
\hline
\end{tabular}

MALDI spectrum of $\mathrm{K}$ coil before deprotection and labeling
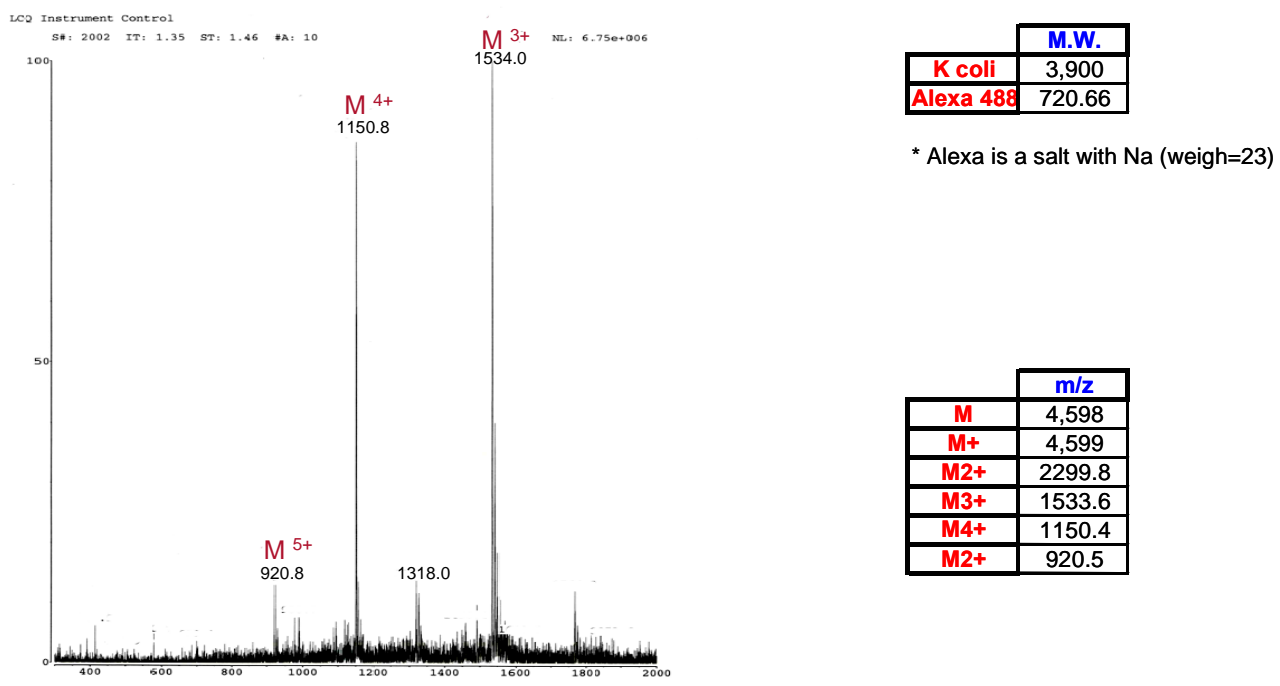

LCQ spectrum of K coil-Alexa 488

Figure 4-13: Mass spectrometric spectra of the synthesized K coil, before and after labeling 
An experiment was designed to demonstrate that the $\mathrm{E}$ coil and $\mathrm{K}$ coil peptides heterodimerize when the $\mathrm{E}$ coil is expressed as a $\mathrm{scFv}$ fusion and when the $\mathrm{K}$ coil is conjugated to Alexa 488. A standard scFv, HyHel10, was expressed alone and as either E coil or K coil fusions. The purified protein of each type, HyHel, HyHel-E coil and HyHel-K coil, was resolved by SDS PAGE gel for Western blot analysis. The proteins were transferred to nitrocellulose membrane and incubated with anti-SV5 and K coil488 simultaneously (Refer to Figure 4-14). Results show that the fluorescently labeled K coil specifically interacts with E coil protein fusions.

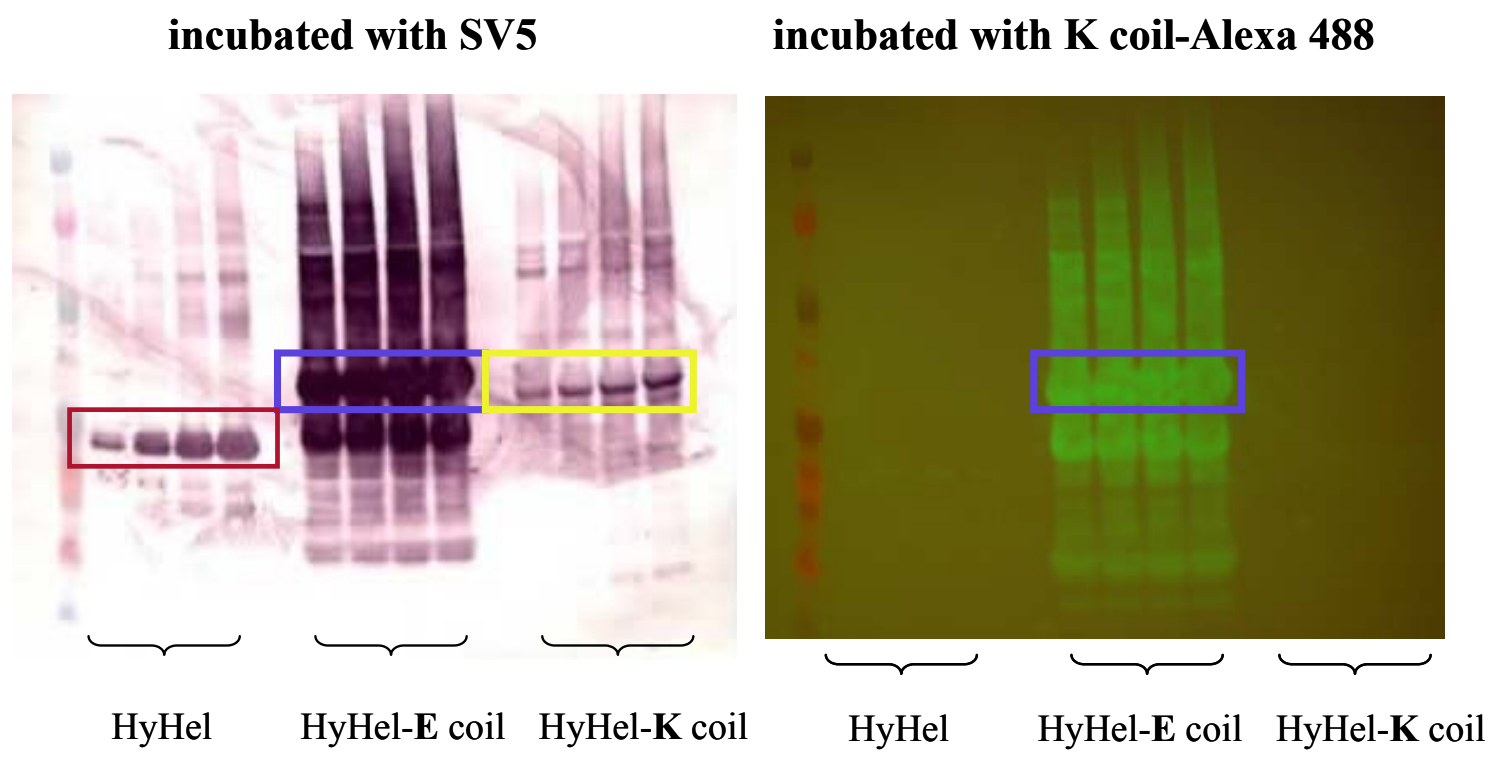

Figure 4-14: Western Blot Analysis of purified single chain-E/K coil fusions. 


\subsubsection{1pEP-scFv-E vector}

The DNA encoding the scFv antibodies produced in each third round selection was cloned into $\mathrm{pEP}$-scFv-E coil vector. PEP-scFv-E ${ }^{6}$ coil vector is derived from $\mathrm{pET} 28$ and allows expression of scFvs as E coil fusion proteins. Expressed scFv-E coil fusions were then directly labeled with K coil Alexa 488 prior to flow cytometry analysis.

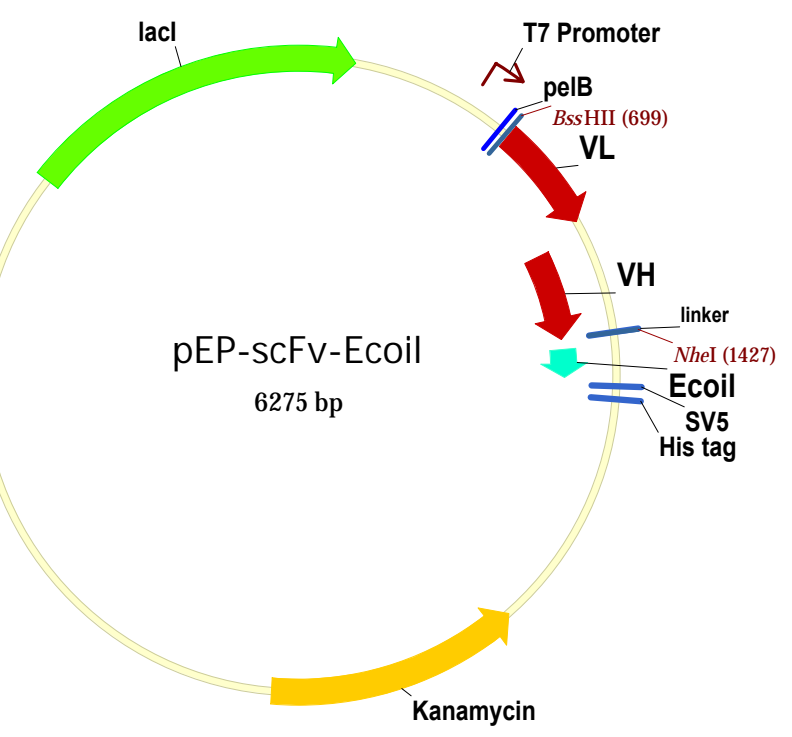

Figure 4-15: pEP-scFv-E vector diagram

\subsubsection{Cloning of the scFvs into the pEP-scFv-E vector:}

DNA encoding the scFvs was recovered from the infected bacterial cells following the third round selection against each of the five target Shewanella antigens. The DNA was digested with BssHII and NheI restriction enzymes and cloned directionally in the BssHII/NheI restricted pEP-scFv-E coil vector. (Refer to Figure 4-16) 


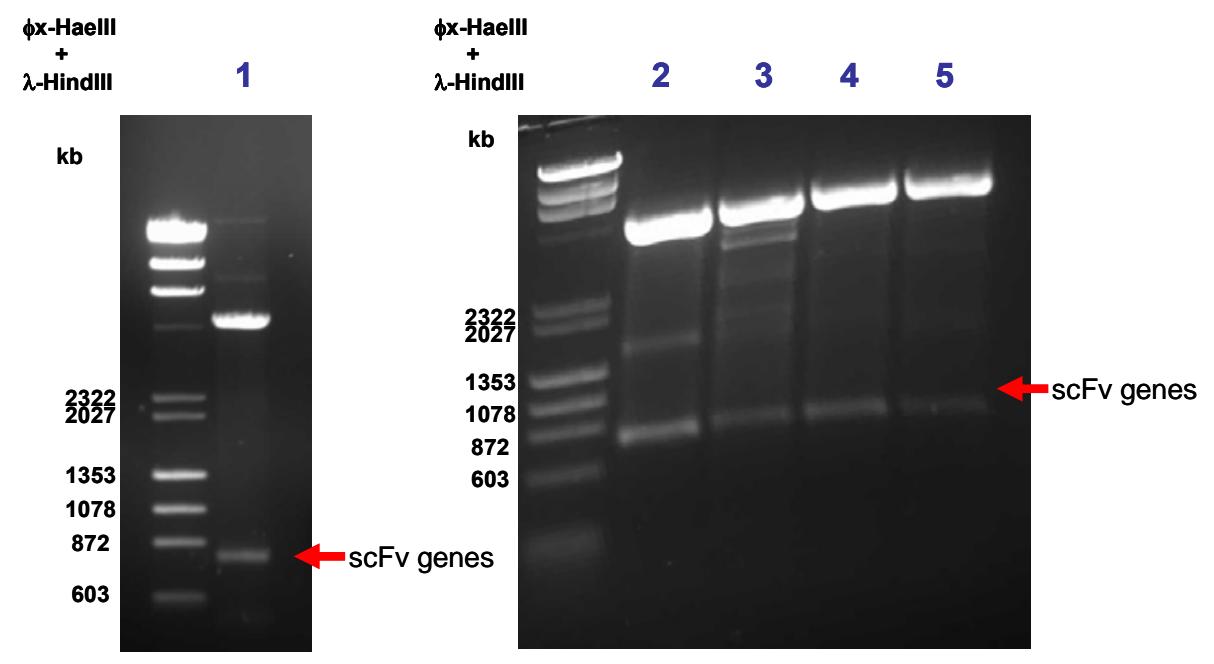

Figure 4-16: BssHII/NheI digestion of scFv DNA from selections against proteins 1, 2,3,4,5.

The fragment size of approximately $800 \mathrm{bp}$, represents full length scFv containing both domains, VL and VH. To get an idea of the single chain diversity in each selection, DNA of 12 randomly picked clones were PCR amplified with $\mathrm{pDpH5}$ ' and Ecoil3' primers. PCR product was digested with BstNI restriction enzyme to produce the DNA fingerprint of each clone. (Refer to Figure 4-17).

The fingerprint of a number of representative clones was determined prior to screening to ensure that the population of scFvs representing the binding population was diverse and did not consist of the same clone (scFvs against antigen \#1 were not fingerprinted at this point). 


\begin{tabular}{|c|c|c|}
\hline antigen \# & different/total & \% different \\
\hline 2 & $3 / 12$ & 25 \\
\hline 3 & $7 / 12$ & 58 \\
\hline 4 & $8 / 12$ & 67 \\
\hline 5 & $10 / 12$ & 83 \\
\hline
\end{tabular}

ScFvs against protein \# 2

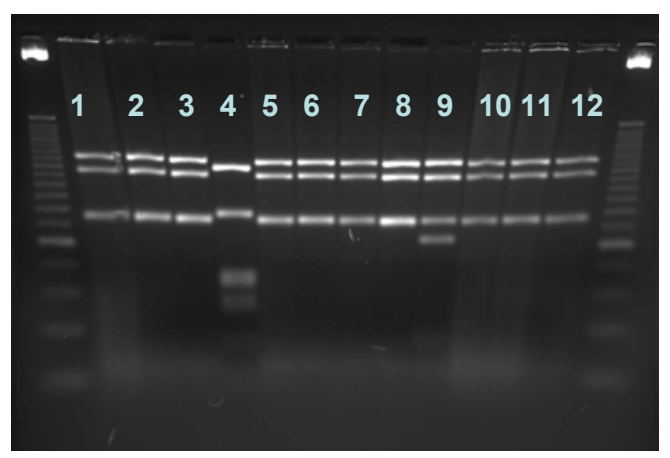

$\underline{\text { ScFvs against protein \# } 4}$

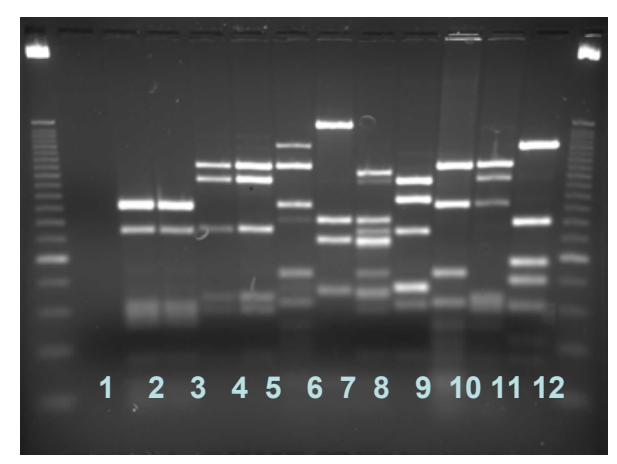

$\underline{\text { ScFvs against protein \# } 3}$

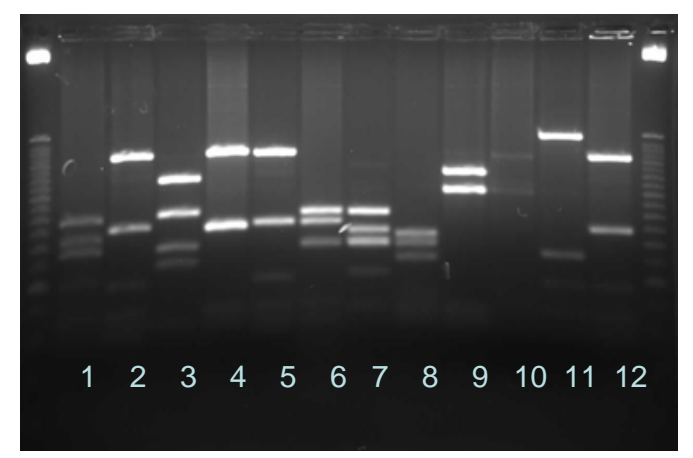

ScFvs against protein \# 5

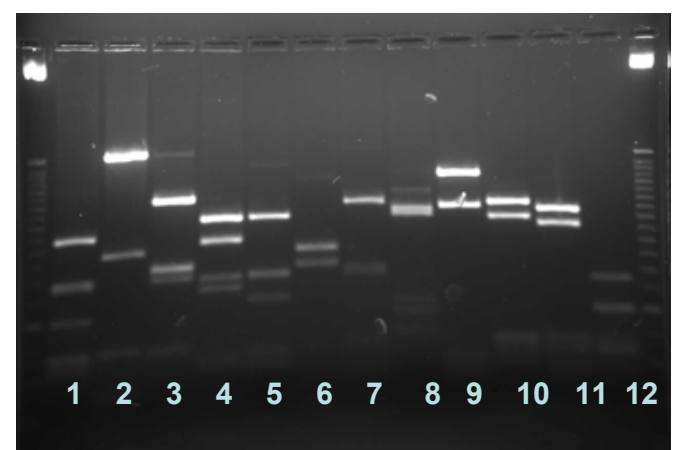

Figure 4-17: Fingerprint of single chains coming from selections against Shewanella antigen targets, $2,3,4,5$ 
The digestion of randomly picked colonies indicated the presence of a large diversity of single chains in all but in the case of protein \# 2 . Because of the large diversity within the potential binding population, 46 individual $\mathrm{scFv}$ clones were expressed and analyzed using multiplex flow cytometry assay (single chains coming from selection against antigen $\# 1$ are not analyzed by this manner)

\subsubsection{Flow cytometry and ELISA analysis:}

Each scFv-E coil clone as expressed in $1 \mathrm{ml}$ of autoinduction media in a 96 deep well plate. Following over night incubation at $25^{\circ} \mathrm{C}$ and $900 \mathrm{rpm}$, the cells were centrifuged to separate them from the media supernatant containing the scFv-E coil fusion proteins. The supernatant containing expressed single chain-E coil fusion was incubated with Kcoil-Alexa488 ${ }^{7}$ to allow the scFv-E coil proteins to become fluorescently labeled (Refer to section 4.6.1). The four bead sets (xMap 129, 142, 135 and 180) were combined into one analytical sample as shown in Figure 4-18B. Each scFv-E coil-K coil-Alexa 488 clone was added to the bead mix and binding events were allowed to take place (Refer toFigure 4-18.C). Each of the samples was analyzed with LSR II flow cytometer (BD Biosciences) (chapter 2: LSRII). 


\section{Components}

↔ neutravidin $\bigcirc$ b-shewanella antigen $\bigcirc$ b- $\alpha$-SV5 $\bigcirc$ b-ubiquitin scFv

\section{Conjugated beads and labeled scFv}
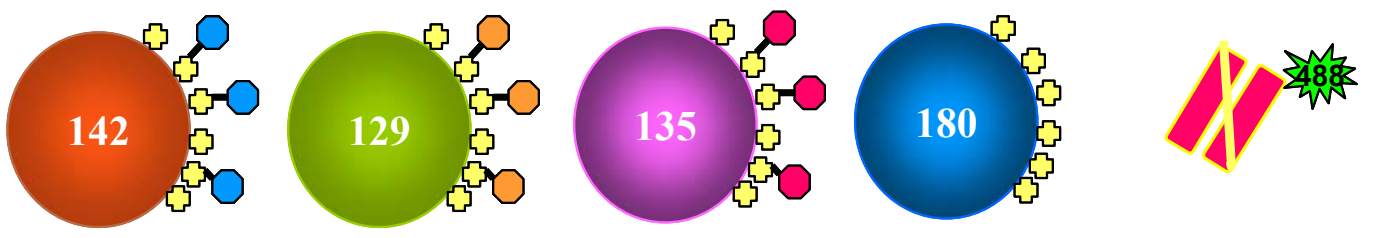

\section{Multiplex Experiment}
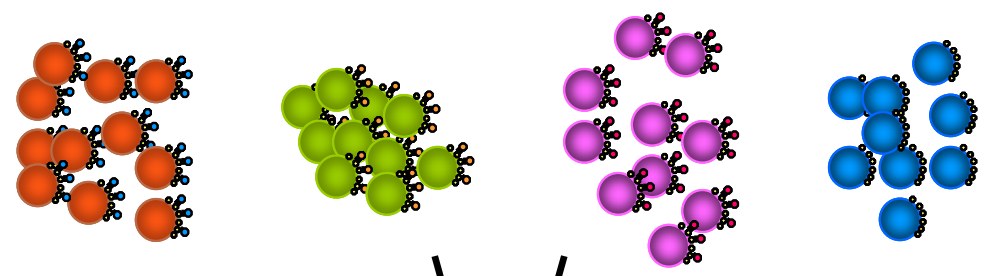

A)

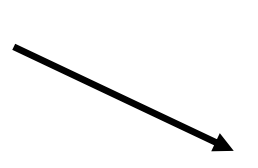

11
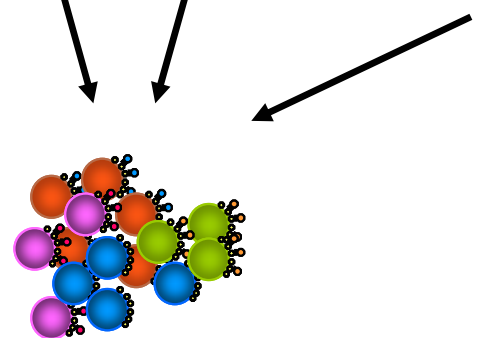

B)

C)
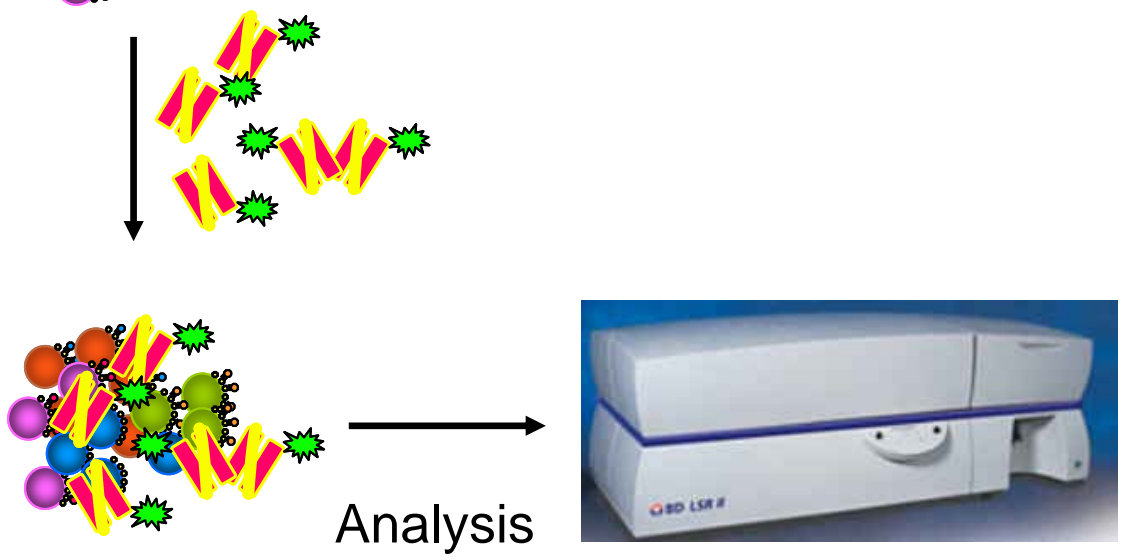

\section{LSRII}

Figure 4-18: Schematic of a multiplex experiment

A) Conjugation of four sets of beads with target antigen, a-SV5, ubiquitin and neutravidin only.

B) Mixing of the four sets of beads

C) Incubation of the beads with the labeled scFvs 
The dye in the beads was excited with a $633 \mathrm{~nm}$ laser separating the bead multiplex in gates after detection with APC-Cy7 and APC filters (Refer to Figure 4-10). A $488 \mathrm{~nm}$ laser excited the fluorescent dye in the beads coming from the single chains and the mean fluorescence of 5000 beads was detected using a FITC filter. Levels of fluorescence measured at the different gates gave us information regarding the different binding events.

\begin{tabular}{|lc|}
\hline population & $\begin{array}{c}\text { FITC-A } \\
\text { Mean }\end{array}$ \\
\hline$\square$ Shew. antigen & 12,296 \\
$\square \alpha$-SV5 & 28,717 \\
$\square$ Ubiquitin & 2,109 \\
$\square$ Empty & 2,568 \\
\hline
\end{tabular}
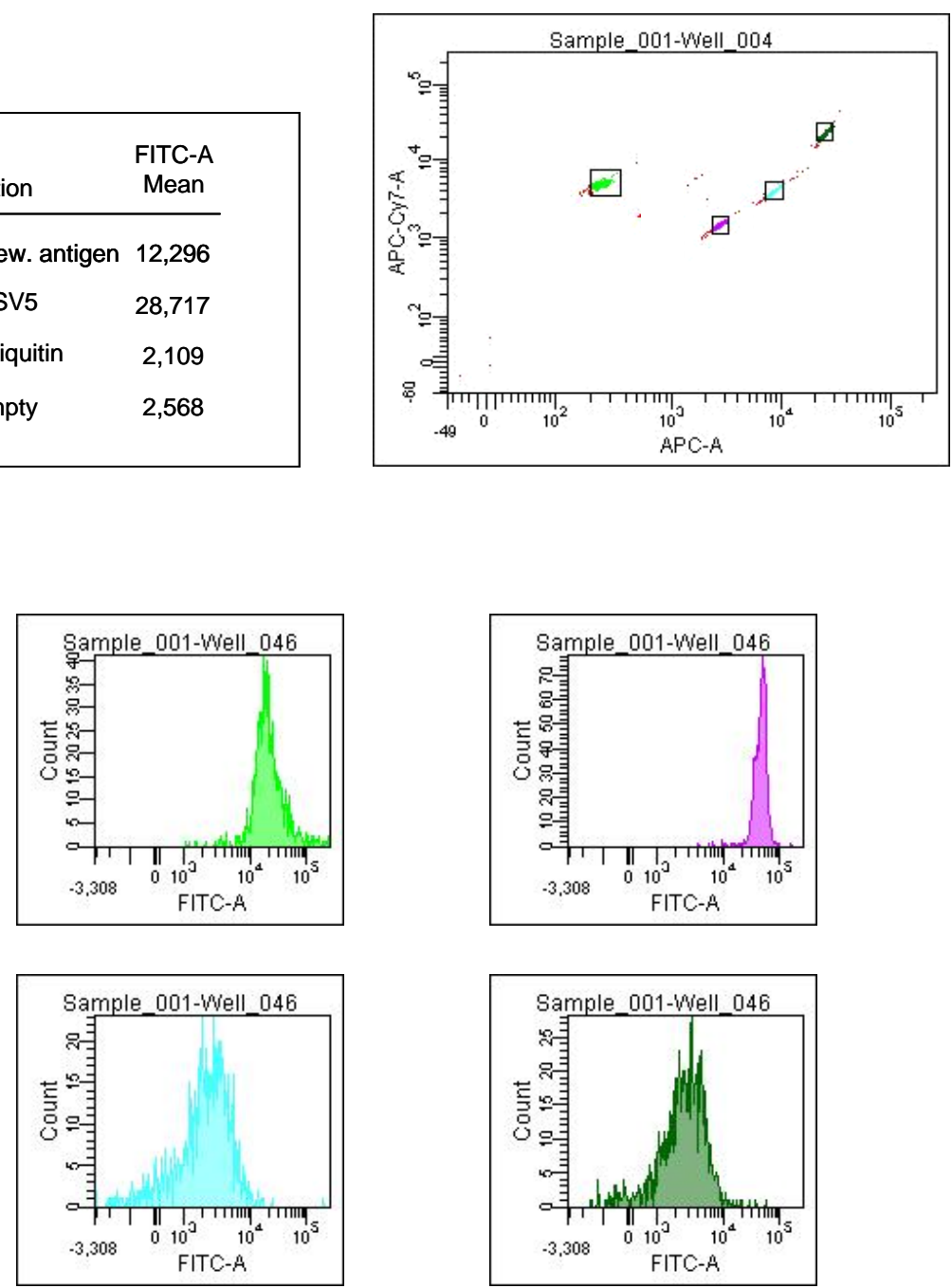

Figure 4-19: Data output coming from LSRII representing a positive binding event for a single chain selected against a Shewanella antigen. 
Fluorescence values coming from the $\alpha$-SV5 conjugated beads, gave an indication of the protein expression level due to the presence of SV5 tag fusion downstream of the scFv-E coil (Refer to Figure 3-19). The background was accounted for by averaging the signal coming from the b-ubiquitin (xMAP 135) and the neutravidin only (xMAP 180) beads. The fluorescent value originating from the XMAP 142 bead set (conjugated to the Shewanella antigen) was normalized by dividing by the corresponding SV5 value. Positive binders were identified as those clones with a fluorescent signal three or more times above the background. (Refer to Table 4-8)

\begin{tabular}{|c|c|c|c|}
\hline Shewanella & population & \multicolumn{2}{|c|}{} \\
\cline { 3 - 4 } antigen & tested & negative & positive \\
\hline$\# 2$ & 46 & 46 & 0 \\
\hline$\# 3$ & 46 & 46 & 0 \\
\hline$\# 4$ & 46 & 36 & 11 \\
\hline$\# 5$ & 46 & 40 & 8 \\
\hline
\end{tabular}

Table 4-8: Analysis of binders by LSR II flow cytometec. Positive corresponds to the number of scFvs with $\mathbf{S} / \mathbf{N}$ three or larger.

No positive binders were identified against Shewanella proteins numbers 2 and 3 . On the contrary, multiple binders were determined for proteins 4 and 5. To confirm the results, the same single chain clones and 46 extra more coming from the selection outputs were tested using ELISA assay. ELISA plates were coated with neutravidin prior to the addition of the biotinylated antigen (chapter 2: SV5 ELISA). ELISA analysis of the scFv binders against antigens \#2 and \#3 gave identical results to flow cytometry analysis; no positive binders were identified Comparison of the flow cytometry and ELISA results produced similar trends in binding activity against the target antigen for antigens \#4 and \#5. 
Those single chain clones that gave a signal of three or more times above background, when analyzed by ELISA, were fingerprinted to check for diversity. DNA originating from these clones was PCR amplified with $\mathrm{pDpH5}$ ' and Ecoil3' primers and the product digested with BstN1 restriction enzyme (Refer to Figure 4-20). (Raw data coming from ELISA and flow cytometry analysis is reported in Appendix to chapter 4). The fingerprint of the ELISA positives shows great diversity of single chains against both antigens \#4 and 5
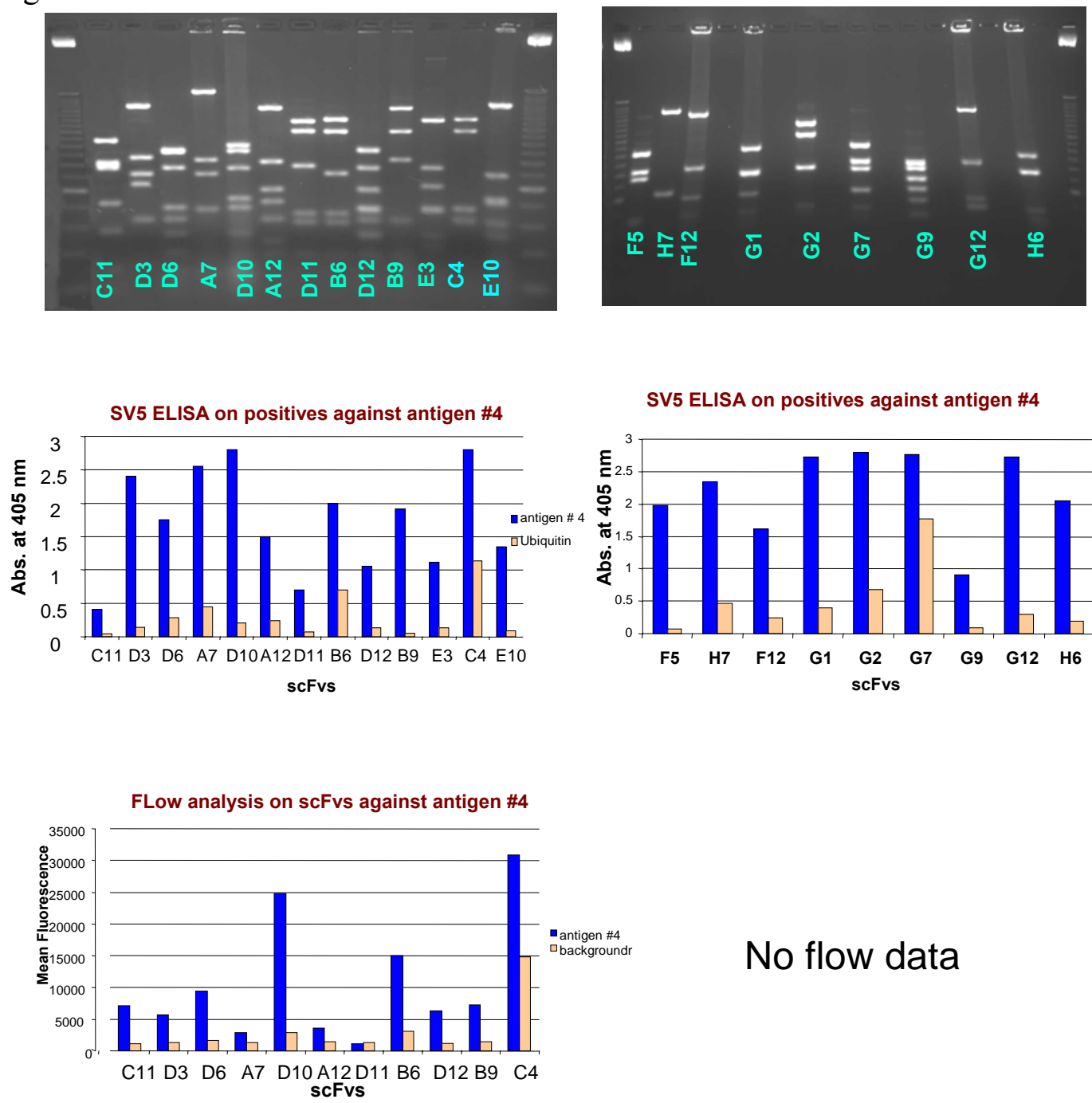

\section{No flow data}

Figure 4-20: Fingerprint of single chains against antigen \#4 that were positive in ELISA shown besides the corresponding ELISA and flow signals. Background is represented by an average of the signals coming from ubiquitin and neutravidin only beads. 96 clones were tested by ELISA, 46 of them also by flow. 


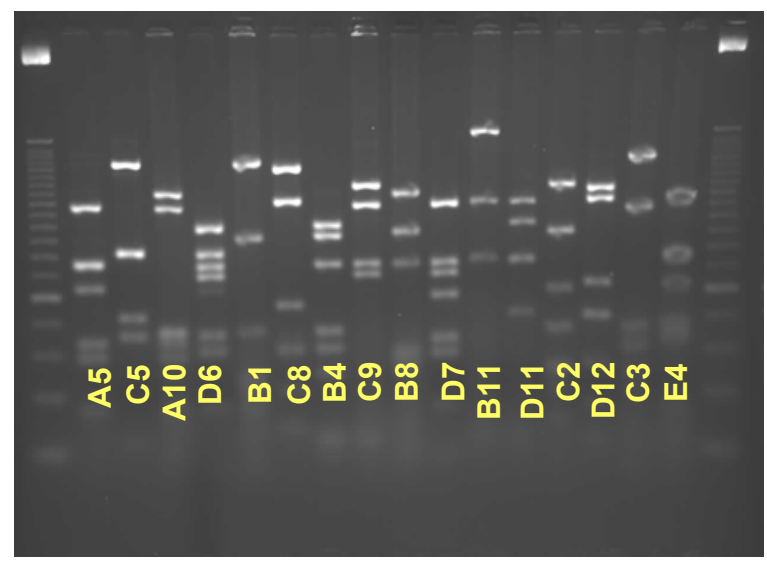

SV5 ELISA on scFvs against antigen \#5

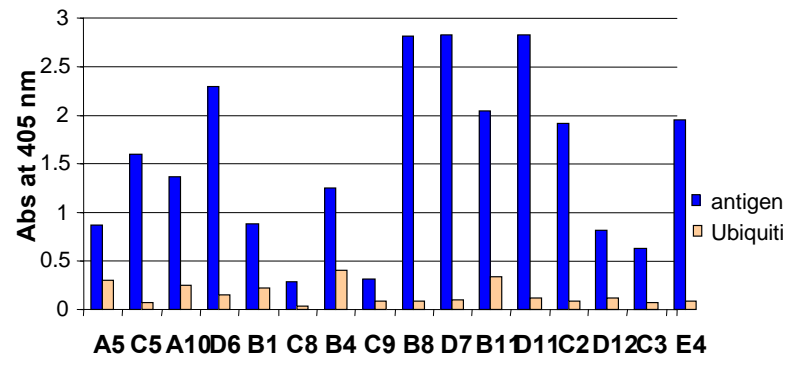

scFvs

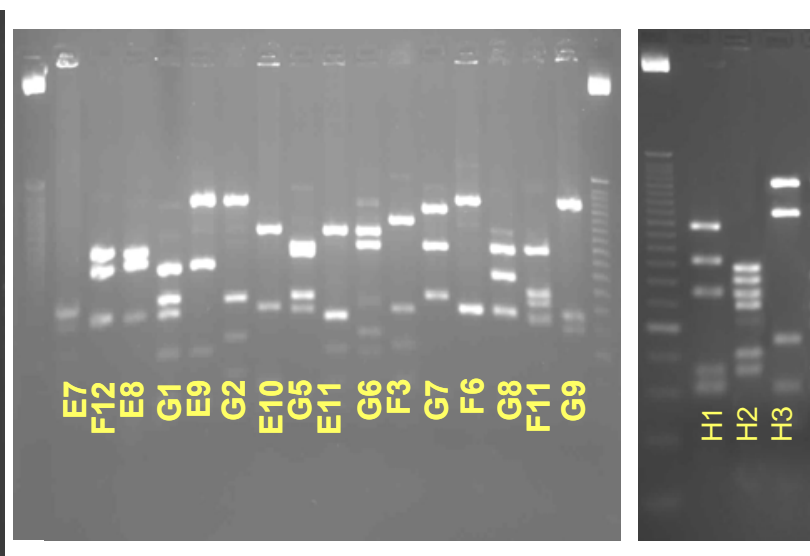

SV5 ELISA on positives against antigen \#5

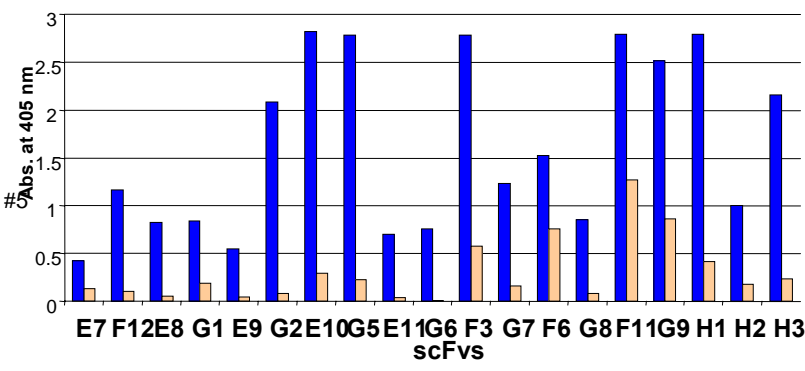

Flow analysis of scFvs against antigen \#5

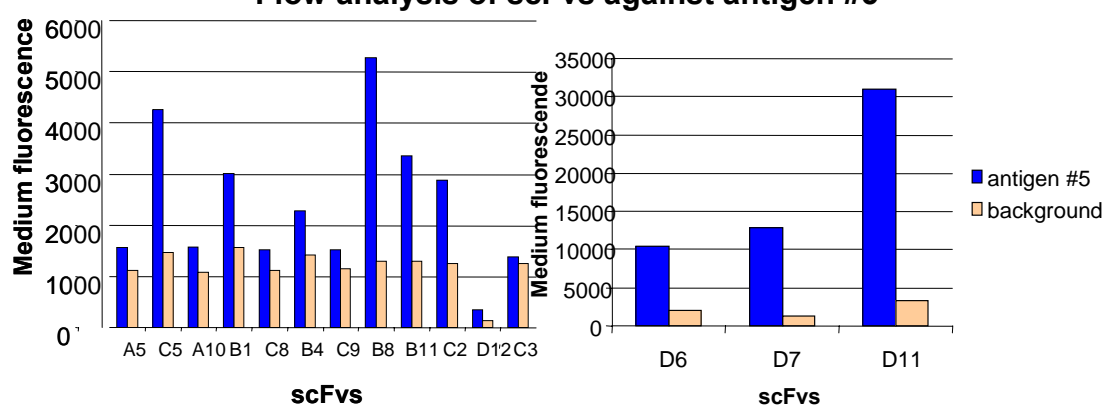

No flow data

Figure 4-21: Fingerprint of single chains against antigen \#5 that were positive in ELISA shown besides the corresponding ELISA and flow signals. Background is represented by an average of the signals coming from ubiquitin and neutravidin only beads. 96 clones were tested by ELISA, 46 of them also by flow. 
96 single chains coming from the selection output against antigen \#1 were analyzed by ELISA (Refer to Appendix, Figure 3) only. A summary of ELISA results for each of the five antigens are shown in Table 4-9. Multiple single chains gave ELISA signals at least three times above that of the negative control (ubiquitin). Those clones that gave positive ELISA signal were fingerprinted to check diversity (Refer to Figure 4-22 ). Diversity of the single chains is large, with very few of them having the same fingerprint.
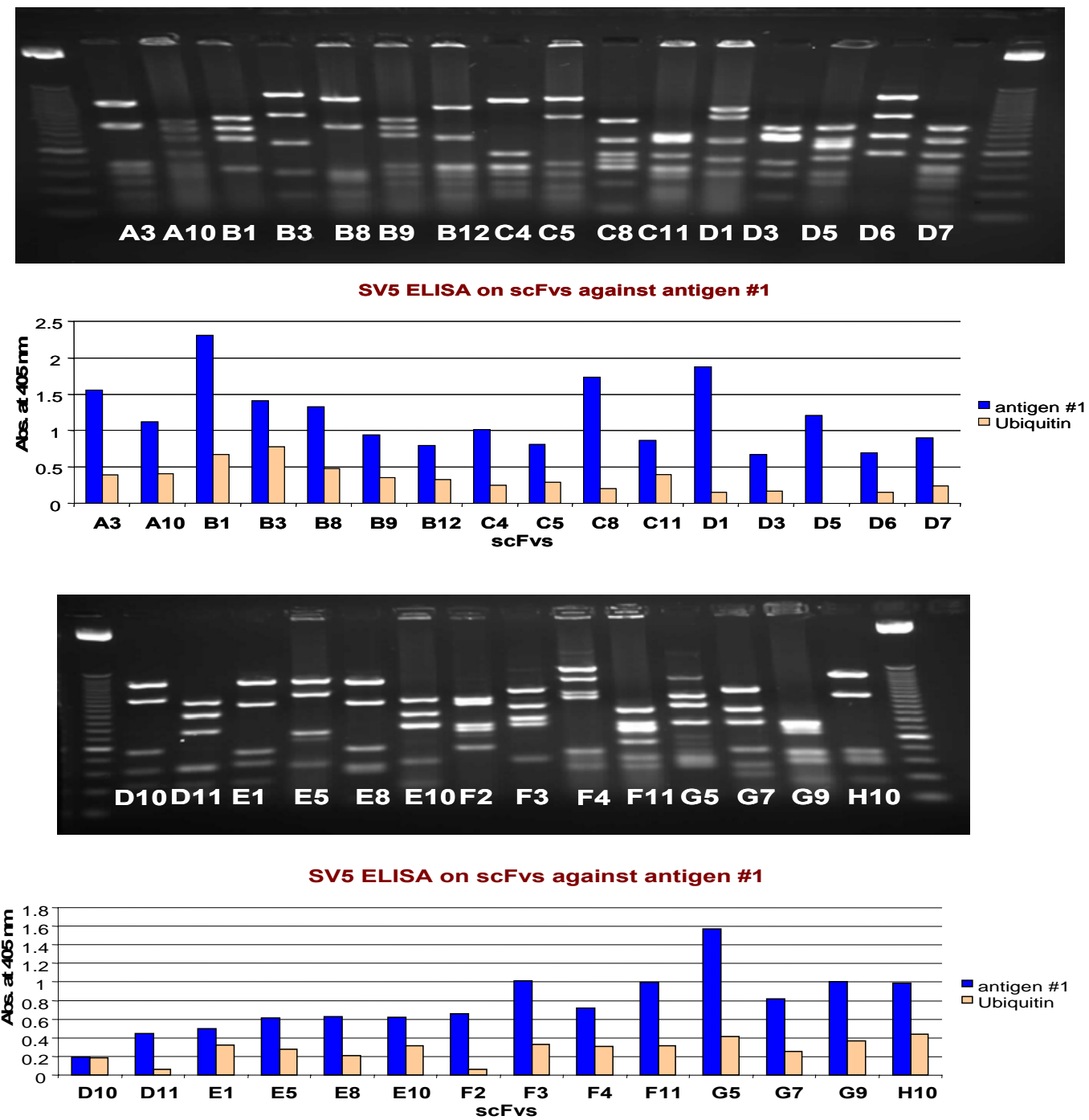

Figure 4-22: Fingerprint of positive single chains against antigen \#1 represented beside the ELISA signals. Each signal corresponds to the average of two replicates. (Neutravidin coated ELISA plate and biotinylated antigen). 


\begin{tabular}{|c|c|c|c|}
\cline { 2 - 4 } \multicolumn{1}{c|}{} & population & positives & \% positives \\
\hline$\# 1$ & 96 & 22 & 22.9 \\
\hline$\# 2$ & 96 & 0 & 0.0 \\
\hline$\# 3$ & 96 & 0 & 0.0 \\
\hline$\# 4$ & 96 & 35 & 36.5 \\
\hline$\# 5$ & 96 & 56 & 58.3 \\
\hline
\end{tabular}

Table 4-9: Summary of results from ELISA assays on Shewanella proteins

The single chains giving positive interaction with antigen \#5 were also checked by ELISA against Shewanella cell extract in place of the protein antigen. Bacterial sample of S. oneidensis MR-1 was provided by Dr Frederickson (Pacific Northwest National Laboratory). Bacteria was grown and stored as indicated (chapter 2: Preparation of Shewanella oneidensis MR-1 cell pellet). Escherichia Coli (E. coli, DH5 $\alpha$ F) was also grown and stored and used as a negative control. Bacterial pellets were thawed an sonicated. Concentration of the total cell protein released to the supernatant was estimated by BCA assay (Pierce). ELISA plates were coated with S. oneidensis and E.coli (Chapter 2: SV5 ELISA) (Refer to Figure 4-23). ELISA steps were followed as previously described.

SV5 ELISA over bacterial extract on positives against antigen \#5

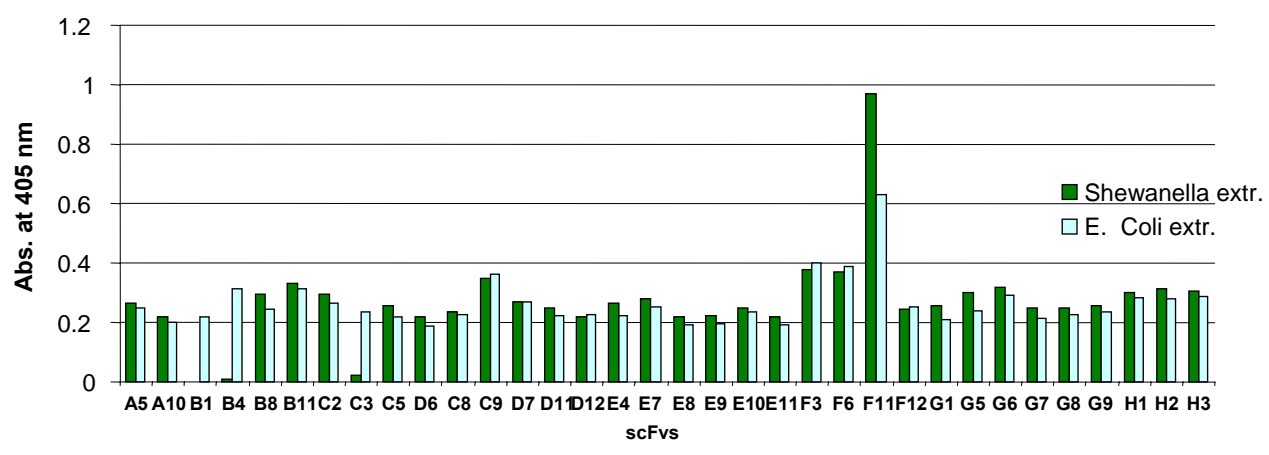

Figure 4-23: ELISA against Shewanella cell extract using scFvs that were positive against antigen \#5. Each signal is the average of two replicates. 
The single chains that gave positive signal when assayed against biotinylated antigen in ELISA did not show specific interaction in the assay with the Shewanella cell extract. Some of the single chains showed, in any case, some specificity for the Shewanella cell extract versus the E. coli one.

The availability of multiple single chains specific for at least three of the Shewanella antigens made the study much more complex. In order to develop the method and with no specific information about the levels of expression of the antigens in the real Shewanella bacteria, the study was continued with two of the Shewanella antigens and the respective single chains. Antigens \#1 and 5 were chosen to continue developing the immunoprecipitation method described in chapter 3. 


\subsubsection{Cloning of the scFvs into the tag vector (pRosa8-scFv-strep)}

Several single chains that were positive against Shewanella antigens \#1 and \#5 were cloned into pRosa8-strep vector in preparation for immunoprecipitation. The single chain candidates were chosen on the basis of being different (identified by fingerprinting) and having specific antigen binding by ELISA. The DNA encoding the single chains was digested with BssHII and NheI restriction enzymes, out of the corresponding pEP-E vector, and directionally ligated to NheI/BssHII digested pRosa8-strep vector (Refer toFigure 4-24).

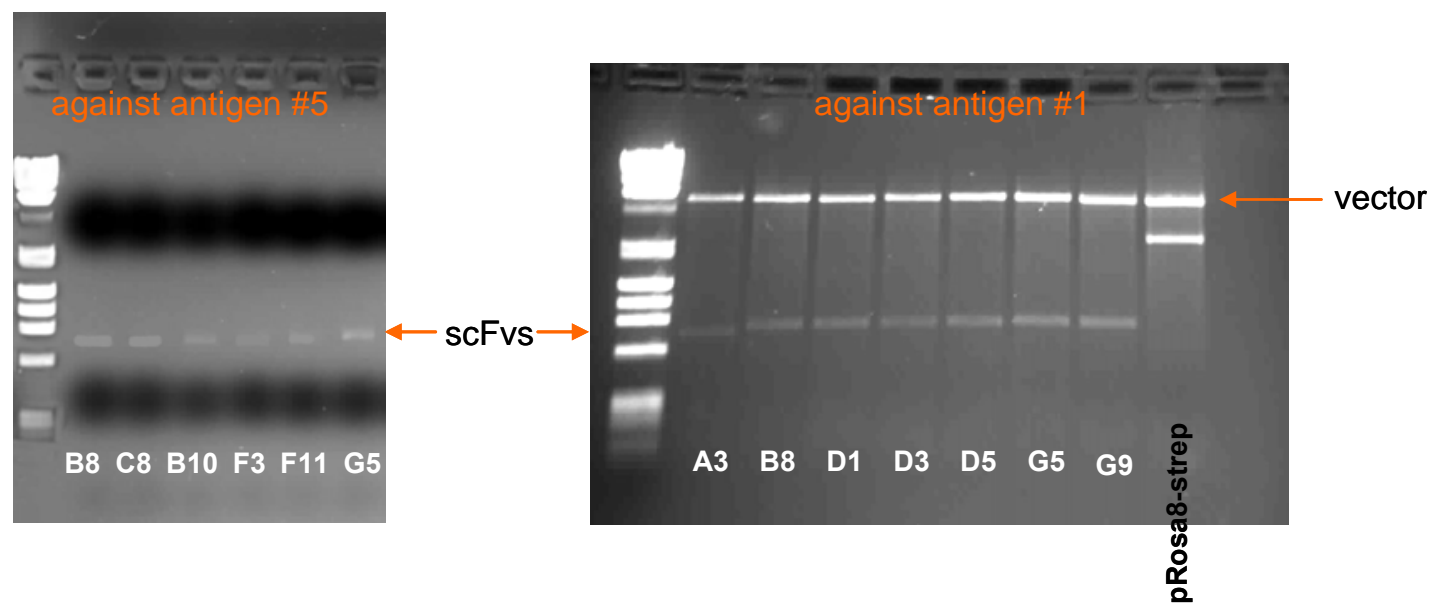

Figure 4-24: BssHII/NheI digested fragments of pRosa8-strep vector and of single chains against antigens \#5 and \#1.

Following ligation and transformation, the DNA was prepared and sequenced using T7 promoter and T7 terminator primers. Sequence data was analyzed using ContigExpress and the alignment of sequences using AlignX (Vector NTI, Invitrogen) software (Refer to Figure 4-25 and Figure 4-26). Seven different scFv candidates were chosen for antigen $\# 1$ and six scFvs for antigen $\# 5$. 


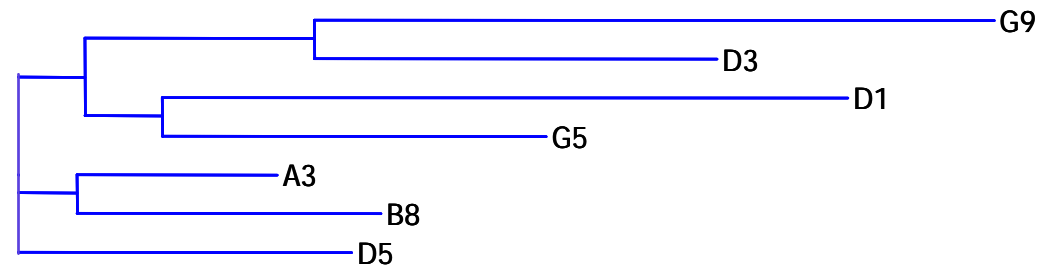

(1) 1

10

20

30

40

50

60

70

80

G9 (1) MEYLLPTAAAGLLLLAAGAALAAAWSHPQFEKGGGSGGGGSGTGGAHAQSVLIQPP-SASGSPGQSVTISCTGTSRDVGG - - - YNHVSWYQKHPGKS

D3 (1) MEYLLPTAAAGLLLLAAGAALAAAWSHPQFEKGGGSGGGSGGTGGAHAQSALIQPP-SASGTPGQRVTISCSGSSSNIG- - - SNYVYWYQLPGTA

D1 (1) MEYLLPTAAAGLLLLAAGALLAAWWHPQFEKGGGSGGGSGGTGGAHADIRMTQSPDSLAVSLGERATINCKSTQSLLYSGNNKNY-LAWYQQKPGQP

G5 (1) -EYLLPTAAAGLLLLAAGAALAAAWSHPQFEKGGGSGGGGSGGTGGAHAEIVLTQSPGTLSLSPGDRATLSCRASQSVGNN - - - - -YLAWFQHKPGQA

A3 (1) MEYLLPTAAAGLLLLAAGAALAAAWSHPQFEKGGGSGGGSGGTGGAHADIVLTQTPATLSVSPGERATLSCRASQSVSSN- - - - - LAWYQQKPGQA

B8 (1) MEYLLPTAAAGLLLLAAGAALAAAWSHPQFEKGGGSGGGSGGTGGAHAEIVMTQSPGTLSLSPGERATLSCRASQSVSSS- - - - YLAWYQQRPGQA

D5 (1) - . - - AAAGLLLLAAGAALAAAWSHPQFEKGGGSGGGGSGGTGGAHADIVMTQSPSTLSASVGDRVTITCRASQSVGSW- . - - LAWYQQKPGKA

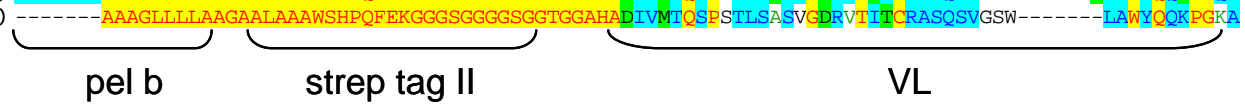

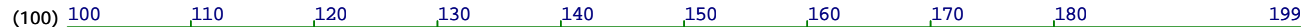
G9 (95) PRLILYDVSKRPSGVSDRFSGSKSGNTASLTISGLQADDEADYYCSSYAGRVIFDIFGGGTKVTVLSGGSTITSYNVYYTKLSSSGTQVQLVQSGAEVKK D3 (94) PKLLIYRNNQRPSGVPDRFSGSKSGTSASLAISGLQSEDEADYYCAAWDDSLNGWVFGGTKLTVLSGGSTITSYNVYYTELSSSGTQVQLVQSGGGLVQ D1 (99) PKLLIYWASTRKSGVPDRFSGSGSGTDFTLTISTLQAEDVAVYYCLQYYS--PPFTFGGGTKVDIKSGGSTITSYNVYYTKLSSSGTQVQLQESGPGLVK G5 (93) PRLLIYDASRRASGIPDRFSASVSGTDFTLTISRLEPEDFAVYYCQQYDR--SPITFGQGTRLEIKSGGSTITSYNVYYTKLSSSGTEVQLVETGGGVVQ A3 (93) PRLLIYGASTRATGIPARFSGSGSGTEFTLTISSLQSDDFAVYYCQQYHN - -WPLTFGGGTKVEIKSGGSTITSYNVYYTKLSSSGTQVQLVESGGGVVQ B8 (94) PRLLIYGASTRATGIPGRFSGSGSGTDFTLTISRLEPEDFAVYYCQQYGS - -APGTFGQGTKVEIKSGGSTITSYNVYYRSYPRAVPRVQLVQSGGGLVQ D5 (86) PKLLMYKASKLERGVPSRFSGSTSGTEFTLTISSLQPDDFATYYCQQYNSY-SPWTFGQGTKVDIKSGGSTITPYNVYYTKLSSSGTEVQLLETGGGLVQ

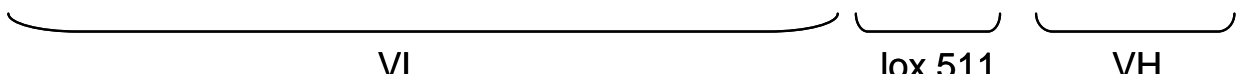

$\mathrm{VL}$

lox $511 \quad \mathrm{VH}$

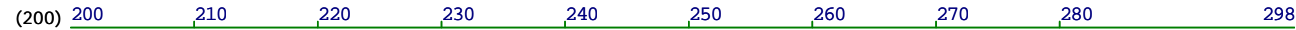
G9(195) PGSSVKVSCKASG- -GTFSSYAISWVRQAPGQGLEWMGIINPPSGGSTSYAQKFQGRVTITADESTSTAYMELSSLRSEDTAVYYCARVRYFDRPVY- - N D3(194) PGGSLRLSCAASG - -FTFNNYAMSWVRQAPGKGLEWVSAISSNGGSTYYADSVKGRFTISRDNSKNTLYLQMSSLRAEDTAVYYCVTS- - - - - -SG - - G DI(197) PSETLSLTCTVSGGSISSSSYYWGWIRQSPGKGLEWLGTMYRSG-SSYRNPSLQSRLTLSMDTSNNQFSLKLTSVTAADTAVYYCARLPRWG - - - - - - W G5(191) PGRSLRLSCAASG - -FTFSSYGMHWVRQAPGKGLEWIGEINHSG-STNYNPSLKSRVTISVDTSKNQFSLKLSSVTAADTAVYYCARRGWYSSGWYPLY A3(191) PGRSLRLSCAASG - -FTFSSYGMHWVRQAPGKGLEWVSAISGSGGSTYYADSVKGRFTISRDNTKNTLYLQMNSLRAEDTAVYYCARAR - - - - - - - B8(192) PGGSLRLSCAASG - -FTFSSYAMSWVROAPGKGLEWVSAISGSGGSTYYADSVKGRFTISRDNSKNTLYLOMNSLRAEDTAVYYCAKLPSSY-YYY- - Y D5(185) PGGSLRLSCAASG - -FTFSSYAMSWVRQAPGKGLEWVSAISGSGGSTYYADSVKGRFTISRDNSENTLYLQMNSLRAEDTAVYYCARGGG - - - - - F- - S

$\mathrm{VH}$

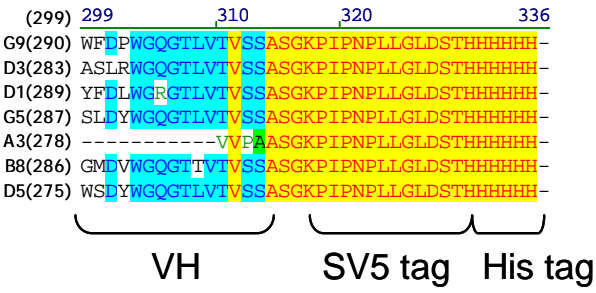

Figure 4-25: Alignment and phylogenetic tree of amino acid sequences of single chains selected against antigen \# 1 


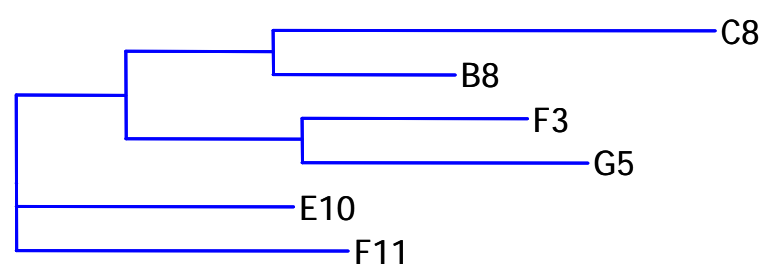

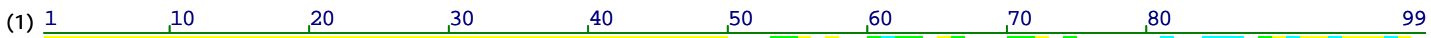

C8 (1) MEYLLPTAAAGLLLLAAGAALAAAWSHPQFEKGGGSGGGGSGTGGAHASSELSQEP-AVSVALGQTVRITCQG - - - - DSLRSSYASWYQQKPGQAPV B8 (1) MEYLLPTAAAGLLLLAAGAALAAAWSHPQFEKGGGSGGGGSGGTGGAHAEIVMTQSPGTLSLSPGERATLSCRAS - - - - QSVSSSHLAWYQQKPGQAPR F3 (1) MEYLLPTAAAGLLLLAAGAALAAAWSHPQFEKGGGSGGGGSGGTGGAHAEIVMTQSPATLSLSPGERATLSCRA - - - - - SQSVSSNLAWFQQKPGQAPR

G5 (1) MEYLLPTAAAGLLLLAAGAALAAAWSHPQFEKGGGSGGGGSGGTGGAHAEIVMTQSPLSSLVTLGQPASISCRSSQSLVHRDGNTYLSWLQQRPGQPPR

E10 (1) MEYLLPTAAAGLLLLAAGAALAAAWSHPQFEKGGGSGGGSSGTGGAHAEIVMTQSPATLSLSPGERATLSCRA- - - - -SQSISRSLAWYQQKPGQAPR

F11 (1) MEYLLPTAAAGLLLLAAGAALAAAWSHPQFEKGGGSGGGGSGGTGGAHAEIVMTQSPLSLPVTLGQPASISCRSSQSLVHSDGNTYLNWFQQRPGQSPR

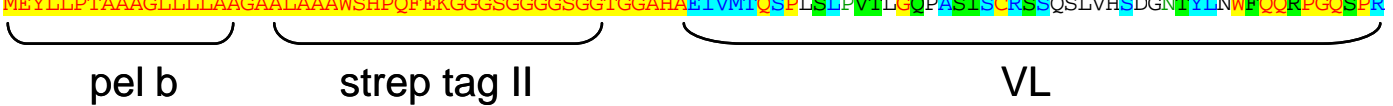

(100) 100

110

120

130

140

150

160

170

180

199

C8 (94) LVIYGKNSRPSGIPDRFSGSTSGNTASLTITRAQAEDEAAYYCHSRDNNNNHLGVFGGRTKLTVLSGGSTITSYNVYYTKLSSSGTQVQLVESGGGLVQP B8 (96) LLIYGASSRATGIPDRFSGSGSGTDFTLTISRLEPEDFAVYYCQQYGSSP- -R-TFGQGTKLEIKSGGSTITSYNVYYTKLSSSGSEVQLVETGGGLVQP F3 (95) LLIYGASTRATGIPARFSGSGSGTEFTLTISSLOSEDFAVYYCQQYNNWPP - - -TFGPGTKLEIKSGGSTITSYNVYYTKLSSSGTQVQLQQSGPGLVKP G5(100) LLIYKVSNRFSGVPDRFSGSGAGTDFTLKISRVEAEDVGVYYCMQATQFPR - - TFGQGTRLEIKSGGSTITSYNVYYTKLSSSGTQVQLQQSGPGLVKP E10 (95) LLIYDASNRATGIPDRFSGSGSGTDFTLTISSLEPEDFAVYYCQQRSNWPR - - TFGQGTKVEIKSGGSTITSYNVYYTKLSSSGTQVQLVQSGAEVKKP F11(100) RLIYKVSNRDSGVPDRFSGSGSGTDFTLKISRVEAEDVGVYYCMQGTHWPP - -WTFGQGTKVEIKSGGSTITSYNVYYTKLSSSGTQVQLVQSGAEVKKP

$\mathrm{VL}$

lox 511

$\mathrm{VH}$

(200) 200

C8 (194) GGSLRLSCAASGFTFSSY - -AMSWVRQAPGKGLEWVS-TISGSGGSTYYADSVKGRFTISRDNSMNTLYLQMNSLRAEDTAVYYCAKQRDYG - . - . - . B8(193) GGSLRLSCAASGFTFSSY - AMSWVRQAPGKGLEWVS-AISGSGGSTYYADSVKGRFTISRDNSKNTLYLQMNSLRAEDTAVYYCAKRVYSSG - . . . F3 (192) SQTLSLTCAISGDSVSSNSAAWNWFROSPSRGLEWLGRTYYRSKWYNDYAVSVKSRITINPDTSKNOFSLOLNSVTPEDTAVYYCARDRGRAP - . . . . . G5(197) SQTLSLTCAISGDSVSSNSAAWNWIRQSPSRGLEWLGRTYYRSKWYNDYAVSVKSRITINPDTSKNQFSLQLNSVTPEDTAVYYCARSRPPCS-STSCYL E10(192) GASVKVSCKASGYTFTGY - YMHWVRQAPGQGLEWMG -WINPNSGGTNYAQKFQGRVTITADKSTSTAYMELSSLRSEDTAVYYCARAGYYYY - - - - - F11(198) GESLKISCKGSGYSFTSY - WIGWVRQMPGQRLEWMG-WINAGNGNTKYSQKFQGRVTITRDTSASTAYMELSSLRSEDTAVYYCAKLRPYYYDSSGYYY

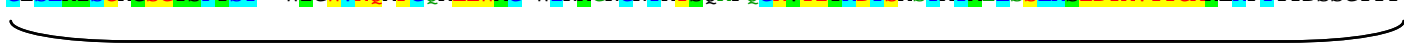

$\mathrm{VH}$

(300) $300 \quad 310 \quad 320 \quad 339$

C8 (283) - - -MDVWGQGTTVTVSSASGKPIPNPLLGLDSTHHHHHH-

B8(283) R- -FDYWGOGTLVTVSSASGKPIPNPLLGLDSTHHHHHH-

F3 (285) YWYFDLWGRGTLVTVSSASGKPIPNPLLGLDSTHHHHHH-

G5(296) GRRFDPWGQGTLVTVSSASGKPIPNPLLGLDSTHHHHHH-

E10(282) G- -TDVWGQGTTVTVSSASGKPIPNPLLGLDSTHHHHHH-

F11(295) YWYFDLWGRGTLVTVSSASGKPIPNPLLGLDSTHHHHHH-

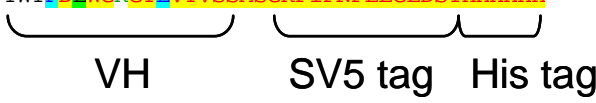

Figure 4-26: Alignment and phylogenetic tree of amino acid sequences of single chains selected against antigen \# 5 
Analysis of the single chains indicated the presence of all the genes expected in the vector with the right sequence. The alignment of single chains indicated the diversity of them, with multiple variations in the sequences of the heavy and light chains from one single chain to another.

\subsection{Discussion}

The work described in this chapter is concerned with the generation of single-chain (scFv) antibodies that selectively bind to TB and Shewanella antigens. Ultimately, these $\mathrm{scFv}$ antibodies will be used to generate scFv-Tag constructs to study protein/protein complexes by immunoprecipitation (IP). Two different types of selections were carried out in order to obtain scFvs for these studies. The first selection experiment used immunotubes coated with the target antigen. This selection was done against protein RV2607 (a TB antigen) and produced two different antibodies, G4 and G12, against the target. Initially, Tuberculosis was the choice organism for IP analysis. More information about protein complexes is needed to be able to understand how TB works and to decipher the protein network. However, in order to develop the new IP method, TB bacteria and proteins were required and the availability of these components was limited. The two anti RV2607 scFvs were not used in the final IP experiments. However, one of the single chains (G4) was used as a model to check the influence of affinity tags in antibody binding (Refer to Chapter 3).

In order to successfully develop scFvs for IP experiments, a different selection method was used and the Shewanella bacterium was adopted as the target organism for these studies. Shewanella is an important organism for remediation and environmental clean-up. However, very little is known about the biological and physiological properties 
of the organism. Developing a new immunoprecipitation method using $\mathrm{scFv}$ antibodies selected against Shewanella proteins could help understand the protein complexes of the organism. One advantage of working with Shewanella includes the fact that the bacteria can be cultured and isolated under Biosafety Level 1 conditions (as the organism poses no risk to human health). In addition, numerous purified proteins were also available from collaborators at the time that these studies were being performed. All of these factors made Shewanella the choice organism to further develop the immunoprecipitation method in place of tuberculosis.

The second selection experiment was carried out using five different Shewanella antigens (proteins renamed \#1, 2, 3, 4 and 5) and selection (panning) in solution. Prior to screening the selection output, fingerprint analysis revealed that the diversity of the scFv output was varied in all cases except for antigen \#2. (Refer to Figure 4-17); indicating that the selection was successful in generating different $\mathrm{scFv}$ clones. Immunotube selection against RV2607 antigen produced only two different selective single chains (G4 and G12). In comparison, 'in solution' panning produced numerous different $\mathrm{scFv}$ antibodies against three of the five antigens under study. Antigen specific binding scFvs were produced against Shewanella antigens \#1, 4 and 5. Positive clones were identified by ELISA and fingerprinted to check the diversity of the $\mathrm{scFv}$ binding population, which proved to be as large as 30,22 and 35 different specific scFv antibodies were generated against the antigen $\# 1,4$ and 5 respectively.

The results given here clearly indicate the superiority of the "in-solution" selection (using magnetic beads) compared to the immunotube method in selecting numerous different $\mathrm{scFv}$ binders. 
In-solution panning utilizes the biotin-neutravidin interaction to capture the target antigen onto the magnetic bead. Biotinylation of the antigen allows the protein to remain in its natural conformation when captured on the neutravidin bead. Furthermore, this allows the $\mathrm{scFv}$ antibodies to interact with the antigen in its native state. During immunotube surface capture of the antigen, the protein undergoes conformational changes that are known to cause protein denaturation ${ }^{8}$.

The uncertainty of the level of coating of antigen in the immunotube could be the reason why more positive binding scFvs were selected and identified using "in solution" panning compared to immunotube selection. Selection "in solution" has proven to yield numerous and more diverse single chain antibodies. Compared to immunotube selections, in solution panning, allows the antigen to remain in its natural conformation state and eliminates background binding of non-specific scFvs.

In addition, the use of the KingFisher ${ }^{\circledR}$ allowed selections to be automated and carried out in high throughput. Automation ensures consistency, reproducibility and eliminates the human error factor. When comparing the "immunotube" to the "in solution" method, the difference is in the number of diverse clones selected ( two for immunotube and more than twenty for in solution) can also be explained by stringency during "in solution" panning. Antigen was decreased 10 times between first and second round, and washes were increased successively from one to five and to fifteen minutes during the "in solution" protocol. On the contrary, the immunotube selection protocol included constant antigen that did not increase the stringency of the selection conditions. The output from the selections carried out against the five Shewanella antigens was analyzed using two different methods, multiplex flow cytometry (scFvs against antigens 
$\# 2,3,4$ and 5) and ELISA (scFvs against antigens \#1, 2, 3, 4 and 5). The scFvs recovered from the third round selection output were cloned into pEP-E vector to produce E coil fusions. The single chains were fluorescently labeled for flow cytometry analysis using $\mathrm{K}$ coil-Alexa 488 that was chemically synthesized and conjugated.

The multiplex flow cytometry method had only been recently developed and apart from the screen that was conducted to generate data for the publication, this was the first time that it had been used to analyze the scFv output from a selection. This method was used to screen the $\mathrm{scFv}$ outputs produced against Shewanella antigens \#2, 3, 4 and 5 and the same clones were also analyzed by ELISA in order to double check the reliability of the flow cytometry analysis.

No scFv binders were identified against antigens $\# 2$ and 3 when analyzed by flow cytometry and this result was confirmed by ELISA. This is unusual for this library, which has usually yielded multiple binders against many different targets, as seen for the other antigens. Re-examination of the antigen (Figure 4-6) shows that there was nothing unusual about these antigens: they were well biotinylated, appeared to be well purified, and were similar in size to some of the other successful antigens.

Out of the 12 randomly picked $\mathrm{scFv}$ clones fingerprinted for antigen $\# 2,10$ were exactly the same (Refer to Figure 4-17). The poor diversity of the scFvs obtained against antigen \#2 explains why no binders were identified for this antigen. It is possible that the same $\mathrm{scFv}$ clone was recovered due to erroneous properties that have allowed it to become enriched during the selection process.

The flow cytometry results were compared to positive ELISA results for antigens 4 and 5. Generally, flow cytometry identified the same positive binders for antigen 
number $4 ; 11$ of the $12 \mathrm{scFvs}$ positive in ELISA were positive by flow cytometry. In contrast, only $8 / 12$ of the 12 ELISA positive binders were identified by flow cytometry analysis for antigen number 5 (refer toTable 4-8). The paper described by Ayriss et al demonstrated that the binding profile (the level of antigen binding) of positive scFvs were identical by ELISA and Flow cytometry. The binding profile of scFvs identified by both ELISA and flow cytometry, in this study, do correlate but the similarities and differences are subtle. This observation could be due to differences in expression levels between the proteins prepared for both the ELISA and flow cytometry experiments. The expressed protein samples were prepared on different days and the expression conditions were different; the length of expression was 3 days for Flow Cytometry and 2 days for ELISA. The length of expression seems to be a critical factor in producing identical yields of protein for obtaining identical binding profiles. Because proteolysis might have degraded some of the expressed scFvs with time, the binding results can only be directly compared between ELISA and flow cytometry when they are corrected for the expression level. The levels of protein expression were not determined for the scFv samples tested by ELISA because it would require doubling the amount of $\mathrm{scFv}$ samples and antigen consumption. In contrast, the multiplexing capability of the flow cytometry allowed the expression levels to be determined at the time of binding analysis. Considering the amount of information that is generated in one analytical sample, the advantages of using multiplex flow cytometry as screen method are numerous and include lower consumption of antigens, time and technical labor. These factors made the screening method very attractive and worth exploring.

An ELISA, using Shewanella cell extract in place of target antigen \#5, was 
carried out using a panel of $\mathrm{scFv}$ antibodies selected against antigen \#5. No specific binders were identified and the negative results could not be concluded because it is not known at what level the amount of 'target antigen $\# 5$ ' naturally exists within the Shewanella cell extract. The success of an ELISA is dependant on both the amount of target antigen available and the affinity of the $\mathrm{scFv}$ for the antigen. If the target antigen \#5 is represented at negligible levels then the ELISA would not be sensitive enough to detect the presence of the target antigen within the cell extract no matter what the affinity of the $\mathrm{scFv}$ was. Furthermore, even if present at reasonable levels, it is possible that insufficient amounts of the antigen were bound to the ELISA plate due to the presence of so many other competing proteins. There was no direct correlation seen between the number of different scFvs recovered and the level of antigen biotinylation.

This study has been successful in producing multiple single chains that are specific against three different Shewanella antigens. The study was continued with 7 and 6 different single chains produced from selections against antigens \#1 and 5 respectively; as they came from populations of scFvs that were the most diverse. The single chains were cloned into pRosa8-strep vector and sequenced. All of the scFv clones were in frame and contained the pelB, strep-Tag, VL, Lox511 recombination site, VH, SV5 and His Tag as expected. The amino acid sequences of the scFvs were aligned and showed the difference in sequence variability between the light and heavy chains, of one scFv compared to the next. These scFv binders will be used to generate scFv-Tag constructs to study protein/protein complexes by immunoprecipitation (IP) 


\subsection{Appendix to Chapter 4}
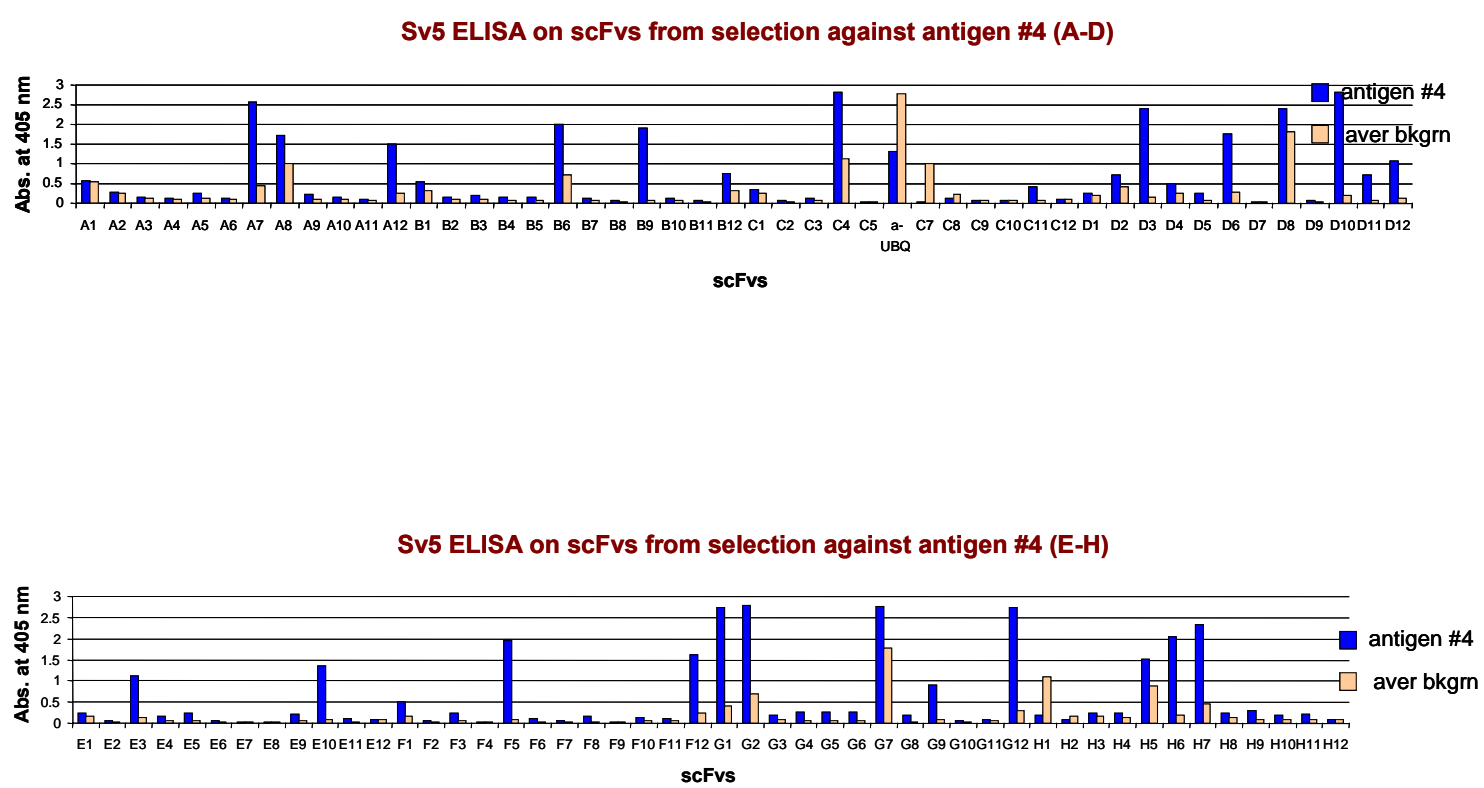

Flow analysis of scFvs against antigen \#4

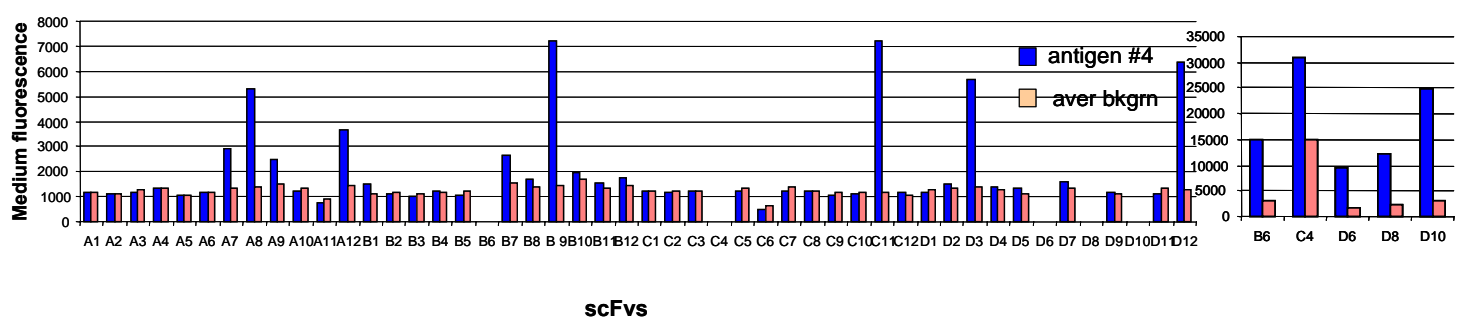

Figure 4-27: Raw ELISA and flow cytometry data corresponding to the scFvs selected against antigen \#4. Flow data has been represented in two different graphs in order to be able to use different scales. 
SV5 ELISA in scFvs from selection against antigen\#5 (A-D)

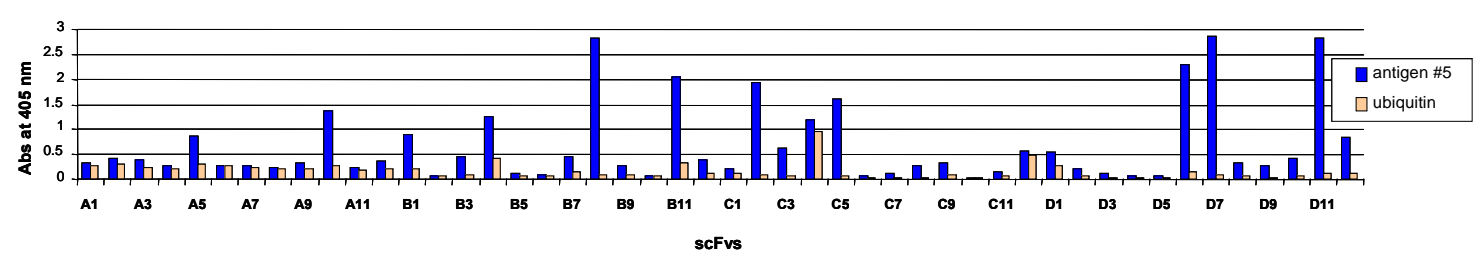

SV5 ELISA in scFvs from selection against antigen\#5 (E-H)

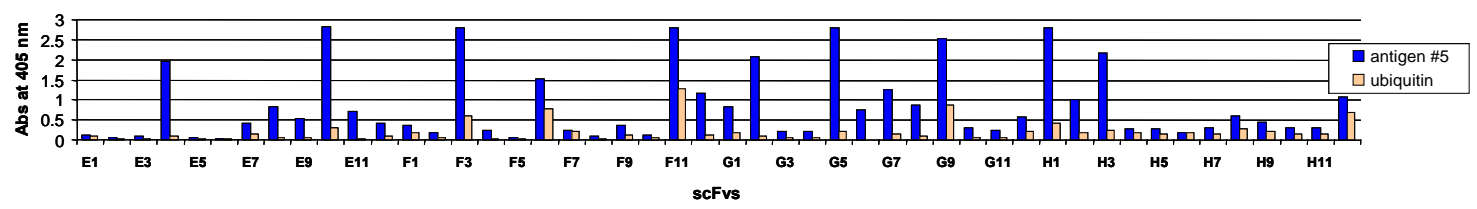

Flow analysis of scFvs against antigen \#5

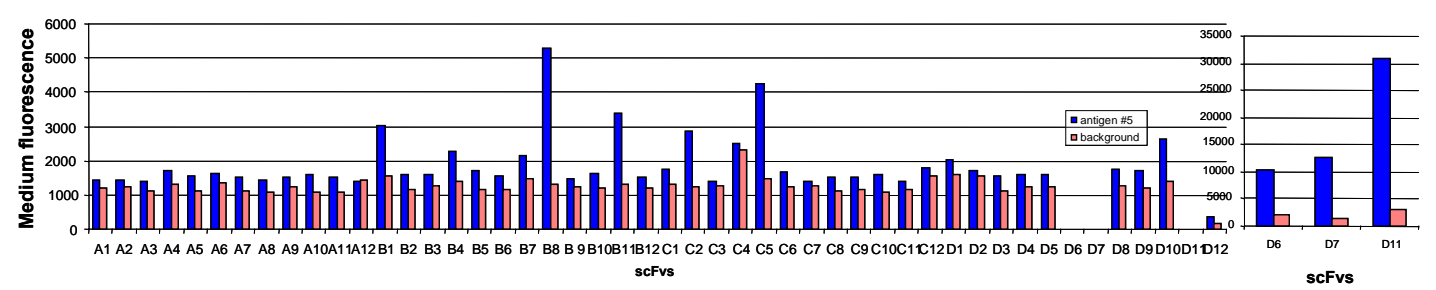

Figure 4-28: Raw ELISA and flow cytometry data corresponding to the scFvs selected against antigen \#5. Flow data has been represented in two different graphs in order to be able to use different scales. 


\section{Chapter 5. Immunoprecipitation of protein complexes}

\subsection{Introduction}

This chapter describes the use of the double tag system, StrepII and SV5 tag fused to single chain antibodies (scFvs), for the capture of immunoprecipitation (IP) complexes. A proof of principle strategy was created to demonstrate how the IP tags, StrepII and SV5, can be used to capture a known antibody/antigen complex comprising two single chains antibodies D1.3, and HyHel10 and their common antigen, chicken lysozyme. The aim of the proof of principle experiment was to demonstrate that the D1.3 $\mathrm{scFv}$ was able to purify in complex with its antigen (Chicken lysozyme) and co-purify with the second anti-lysozyme scFv, HyHel10. A scheme of the proof of principle experiment is shown in Figure 5-1.

The double tag purification system was applied to a real IP experiment using Shewanella oneidensis MR-1 (S. oneidensis) extract for the capture of protein-protein complexes. $\mathrm{ScFv}$ antibodies specific for $\mathrm{S}$. oneidensis antigens \#1 and \#5 (Refer to chapter 4) were selected (using phage display), expressed and purified as SV5 and strep tag fusions (refer to Chapter 3 section 3.4.1). These selected scFvs were used in parallel IP experiments in an attempt to generate protein-protein complexes from Shewanella cell extract. The results and conclusions of the S. oneidensis IP capture experiments are described here. 
$\bigcirc \rightarrow$ lysozyme

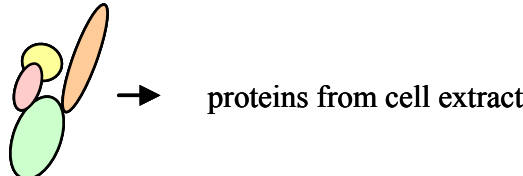
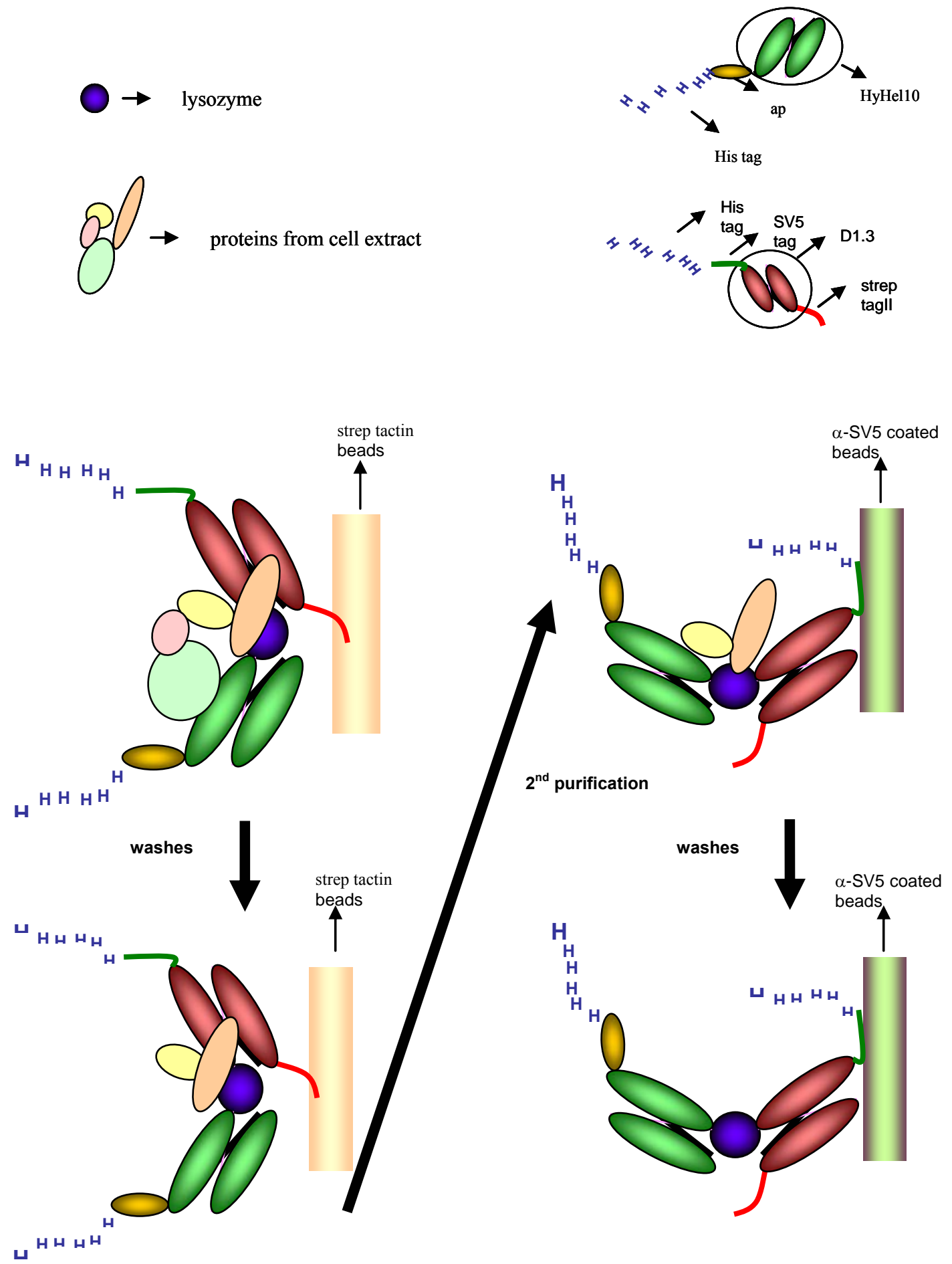

Figure 5-1: Schematic of the double tag purification process. 


\subsection{Immunoprecipitation of D1.3-lysozyme and Hyhel-10 using strep tag II and SV5 tag}

As described in Chapter 3 section 3.4.2, the scFv tag clones were prepared for use in these IP, proof of principle experiments. The Strep and SV5 Tags were cloned at the amino $(\mathrm{N})$ and carboxyl (C) termini of the scFv gene, encoding D1.3, respectively. The gene encoding alkaline phosphatase (AP) was cloned at the $\mathrm{C}$ terminus of HyHel10. The HyHel10-AP clone did not contain either StrepII or SV5 tag.

Both of the scFv fusion proteins, Strep-D1.3-SV5 and the HyHel10-AP, were expressed and recovered from the cell pellet using the Emulsiflex Homogenizer (Avestin). The cell extract was filtered and each $\mathrm{scFv}$ protein was subsequently purified using Ni-NTA chromatography (via the hexa-Histidine tag). Generic elution conditions were used, so the proteins were not very pure. However, for the purposes of the experiments described here, this was not too important, as the purified protein was mixed with E. coli extract to mimic more closely a real IP.

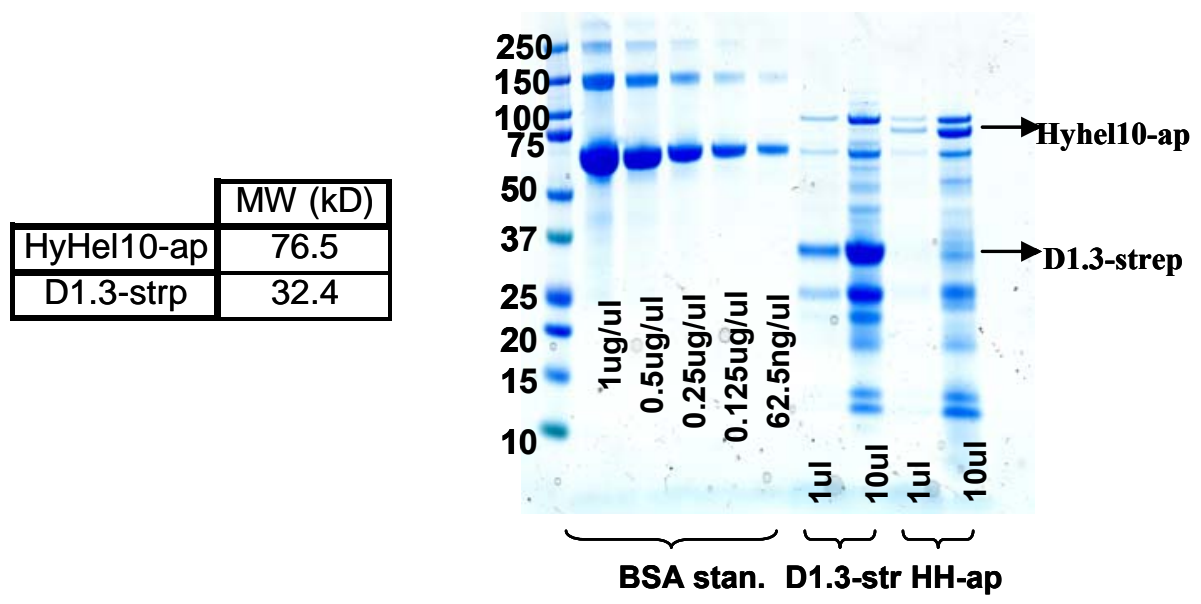

Figure 5-2: Gel quantitation of the purified single chains. Proteins were recovered at a concentration of $0.3 \mu \mathrm{g} / \mu \mathrm{l}$ (Strep-D1.3-SV5) and $0.1 \mu \mathrm{g} / \mu \mathrm{l}$ (HyHel10-AP). 
The IP complex was created by mixing Escherichia coli (E. coli) cell extract with each purified scFv (Strep-D1.3-SV5 and HyHel10-AP) and lysozyme. The sample was incubated with prepared StrepTactin beads (Refer to chapter 2: StrepTactin purification) rotating for one hour at $4^{\circ} \mathrm{C}$. Three samples were prepared in parallel in order to compare the purification of IP complexes following two separate single and one double tag purification.

Two different types of single tag purification were performed. The first single tag purification utilized the Strep tag II using micro columns containing Strep-Tactin and mild elution conditions (Refer to chapter 3, section 3.5). In contrast a second, single tag, purification was carried out using SV5 tag, using Protein A tips (Phynexus) coated with anti-SV5 antibody, and harsh elution conditions (Refer to chapter 2: SV5 purification using protein A tips).

In the case of double tag purification, the mixture of single chains (D1.3 and HyHel10), lysozyme, cell extract and beads was incubated with rotation for an hour at $4^{\circ} \mathrm{C}$. The protein complex was first purified using the Strep tag II in D1.3 and the eluted fraction was recovered and carried through a second purification using protein A tips (Phynexus) coated with $\alpha$-SV5 antibody. The proteins recovered in the eluted samples were analyzed by SDS-PAGE (4-12\% Bis-Tris) and stained with silver for visualization. 


\begin{tabular}{|c|c|}
\cline { 2 - 2 } \multicolumn{1}{c|}{} & MW (kD) \\
\hline HH-ap & 76.5 \\
\hline D1.3-strp & 32.4 \\
\hline$\alpha$-SV5 & $25(\mathrm{LC})$ \\
\hline & $50(\mathrm{HC})$ \\
\hline Lysozyme & 14.3 \\
\hline
\end{tabular}
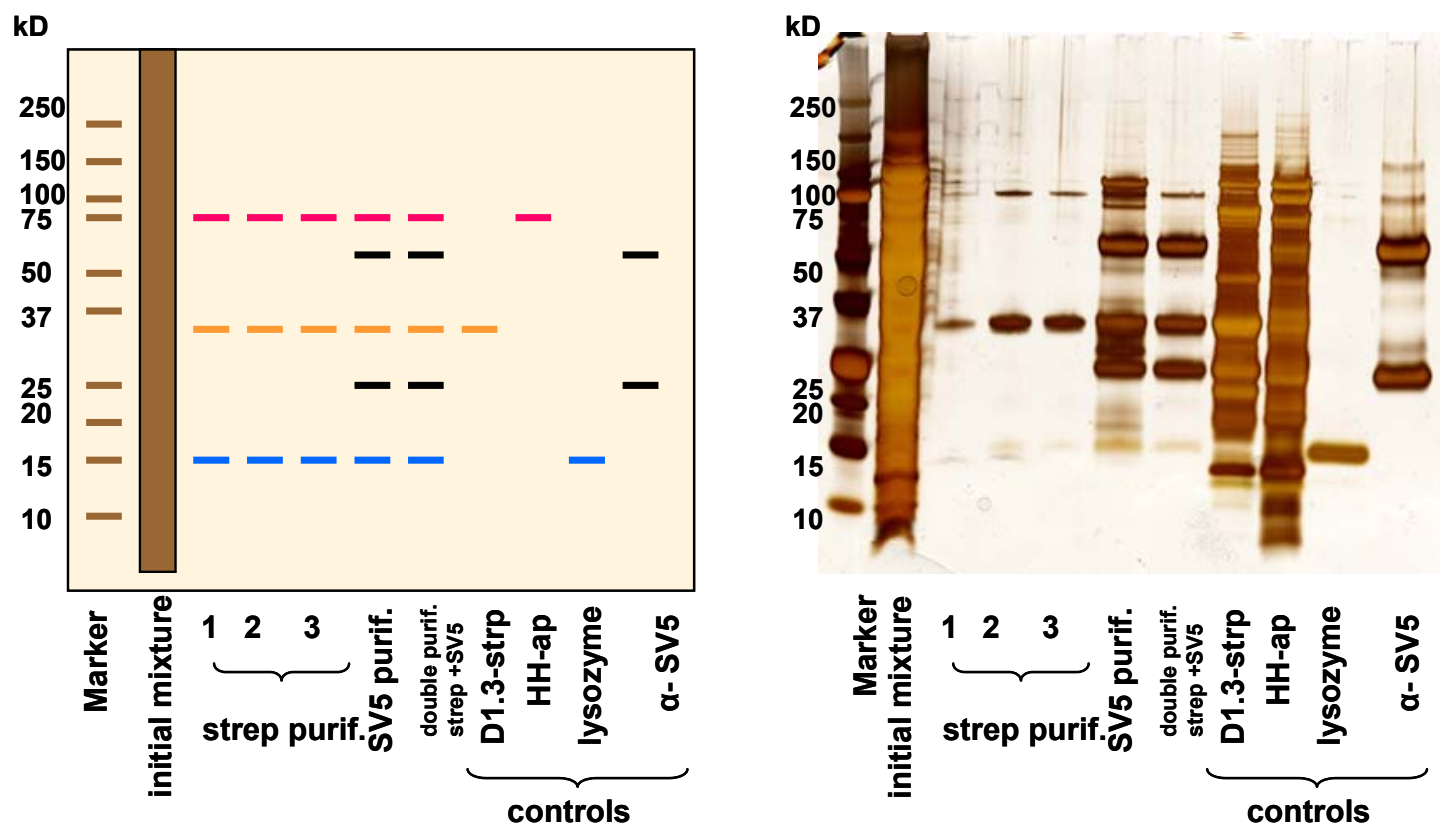

Figure 5-3: SDS Page analysis of model complex immunoprecipitation, comparison of double and single tag purifications. Left figure is the expected bands, right hand one is the actual experiment. $1 \mu \mathrm{g}$ of each scFv and equimolar amounts of lysozyme was added to $50 \mu \mathrm{l}$ of cell extract. Elution from strep Tactin matrix was in $100 \mu \mathrm{l}$ and in $30 \mu \mathrm{l}$ from the $\alpha-S V 5$ column. Samples were loaded in equal volumes $(10 \mu \mathrm{l})$. Controls of each component were added HyHel10 $(1 \mu \mathrm{g}), \alpha-S V 5(2.5 \mu \mathrm{g})$, D1.3 $(3 \mu \mathrm{g})$. Gel was developed by silver staining (chapter 2: silver staining) 
Results from single and double purification are represented in the silver stained protein gel (Refer to Figure 5-3). The initial E.coli cell extract sample shows the large density of proteins present. Samples 1, 2 and 3 show the proteins present in the eluted fractions following single tag purification with strep tag II. The purification is very clean and the only bands present in the eluted samples correspond to the expected products, HyHel10-AP, Strep-D1.3-SV5 and chicken lysozyme. Following single tag purification using SV5 tag (Refer to SV5 purif.) the three components of the model complex, (HyHel10-AP, Strep-D1.3-SV5 and lysozyme) are visible, in addition to bands corresponding to the anti-SV5 heavy and light chain (originating from the anti-SV5 antibody on the Protein A tips). Results show that the single tag purification with SV5 tag is not as clean as the single tag purification using Strep tag II. In contrast, double tag purification produced very clean and well defined bands corresponding to the 3 IP components. This type of purification gave a very clean and concentrated product contained within a small volume (30ul). The proteins shown in lanes 8, 9, 10 and11 correspond to the positive control samples included to allow identification of the three components (HyHel10-AP, Strep-D1.3-SV5 and chicken lysozyme) used in this experiment. 


\subsection{Immunoprecipitation of S.oneidensis MR-1 protein complexes}

\subsubsection{Expression and purification of scFv-Tag fusions:}

Four scFvs (G5, A3, B8-1 and D5) selected against S. oneidensis protein \#1 and three (B8-5, F3 and F11) selected against. S. oneidensis protein \#5 were fused to both Strep tag II (N terminus) and SV5 Tag (C-Terminus) by directional cloning into pRosa8strep (Refer to chapter 3, section 3.4.1). The scFv-tag fusions proteins were expressed within autoinducing or 2xTY-IPTG media (Refer to chapter 2: protein expression). No differences in expression yields were observed depending upon which type of expression media was used (data not shown). The $\mathrm{scFv}$ proteins were extracted from the periplasm (Refer to chapter 2: periplasmic extraction) and purified by affinity chromatography, using the hexaHistidine tag. Two different formats of Ni purification were tested: 1) NiNTA column purification with a large column bed (500 $\mu 1)$ and 2) Ni beads in tips with a small column bed (5ul, Phynexus tips).

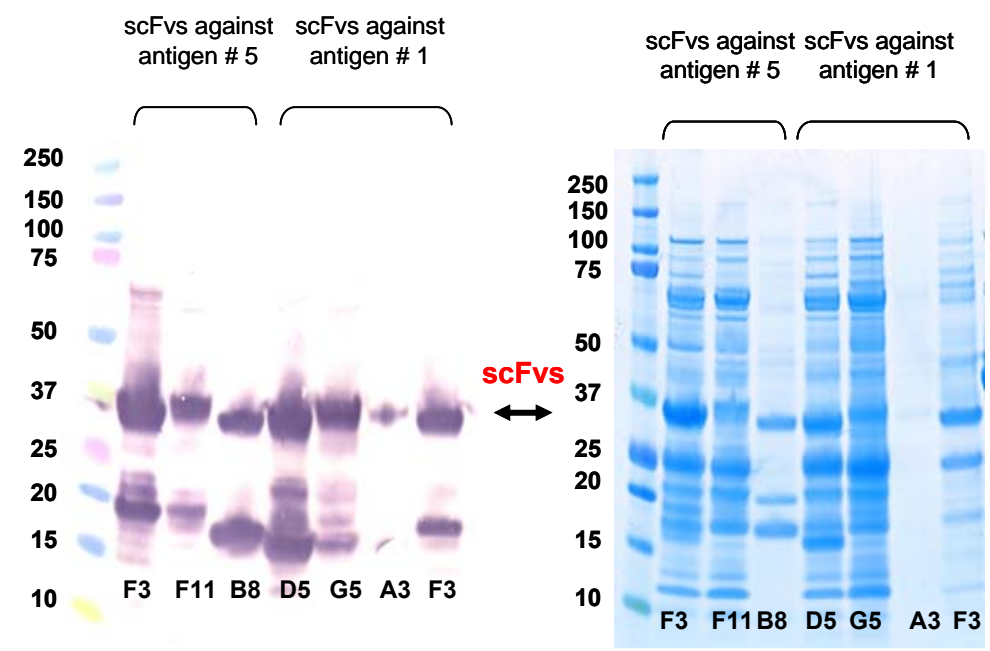

Figure 5-4: Purified scFvs against antigens \# 5 and \#1 in Western Blot and gel code stained gel. B8 and A3 samples purified using Ni-NTA tips and all the others with agarose beads in column. 
The purification results material was analyzed by SDS-PAGE and the proteins were visualized following stain with Gel Code Blue Stain Reagent (PIERCE). Following SDS PAGE separation, the proteins were transferred to nitrocellulose membrane and the scFv proteins were identified by Western Blot with anti-SV5 antibody. (Refer to Figure 5-4). Due to the large volume of periplasmic extract, column purification was the method most regularly chosen.

The S. oneidensis MR-1 bacteria were cultured (Refer to chapter 2: Amplification of S. oneidensis MR-1) and the whole cell extract was prepared by homogenization (Emulsiflex homogenizer, Avestin). The total protein concentration of the whole cell extract was determined using the Bradford assay (BioRad). However, the concentration of each of the two target antigens \#1 and \#5 within the whole cell extract was not known.

\subsubsection{I.P. Experiment \#1:}

The aim of the experiment was to determine whether two different sets of scFvs specific for S. oneidensis antigens \#1 (clones A3, B8-1, D5 and G5) and antigen \#5 (clones B8-5, F3 and F11) could be used collectively to generate and capture IP complexes from S. oneidensis cell extract.

The first set of scFvs comprised those four different clones generated against $S$. oneidensis protein \#1; A3, B8-1, D5 and G5. Whereas the second scFv set consisted of three different clones specific for S. oneidensis protein \#5 (B8-5, F3 and F11). Each of the two different scFv sets was tested, in parallel, for their ability to form IP complexes with S. oneidensis cell extract. The mixture of each $\mathrm{scFv}$ candidate was incubated with Shewanella cell extract for two hours at $4^{\circ} \mathrm{C}$ and then with StrepTactin beads, rotating overnight at $4^{\circ} \mathrm{C}$. The IP complex was purified using the double tag (strep tag II and SV5 
tag) system previously described (Refer to Chapter 3). The flow through and eluted proteins were resolved by SDS-PAGE and analyzed by Western Blot analysis (data not shown).

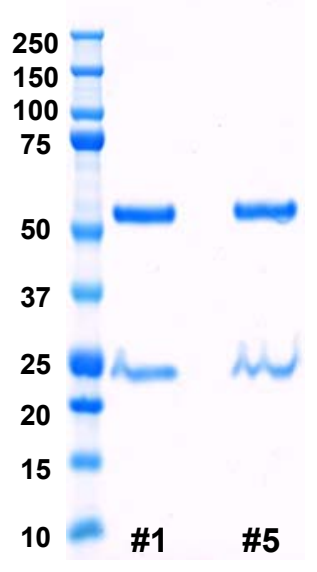

Figure 5-5: SDS PAGE gel of I.P experiments. First lane marker, second, I.P. experiment using protein \#1 and specific scFvs, third, I.P. experiment using protein \#5 and specific scFvs. $1 \mu \mathrm{g}$ of each scFv was added to $10 \mathrm{ml}$ of $S$. oneidensis $(1 \mathrm{mg} / \mathrm{ml})$. Samples were incubated over night with strep Tactin beads

Results of SDS PAGE analysis show that the only proteins present in the eluted fraction are the two proteins corresponding to the Heavy (50kDa) and Light (25kDa) chains of the anti-SV5 antibody used to capture the IP complex. These proteins were also observed in the proof of principle experiment described previously. No other proteins were visible. Due to this negative result, all fractions produced at each step of the IP process were checked for the presence of scFv-Tag protein by SDS PAGE and Western blot analysis (data not shown). None of the 7 different scFvs, or any other protein antigens, was detected. 


\subsubsection{I.P. Experiment \#2:}

The technical procedure of IP experiment \#1 was modified by reducing 1) the $S$. oneidensis cell extract volume from $10 \mathrm{ml}$ to $1 \mathrm{ml}$ and 2) the incubation time with the StrepTactin beads from overnight to 1 hour at $4^{\circ} \mathrm{C}$. The same two scFv sets were prepared as used in experiment \#1. However samples were also prepared, for each $\mathrm{scFv}$ set, where the $S$. oneidensis cell extract was spiked with the target $S$. oneidensis nonbiotinylated antigen \#1 (clones A3, B8-1, D5 and G5) or \#5 (clones B8-5, F3 and F11). In addition, each $\mathrm{scFv}$ set was mixed with $S$. oneidensis cell extract spiked with the nontarget, non-biotinylated antigen.

Each scFv was added to $S$. oneidensis cell extract. The scFv protein set and $S$. oneidensis cell extract were mixed and then either PBS, target or non-target antigen was added depending upon the sample being prepared. The samples were incubated with the StrepTactin beads for one hour at $4^{\circ} \mathrm{C}$ and the resulting complexes were purified as described in section 5.1. A summary of the individual samples prepared and tested in this experiment are shown in Table 5-1

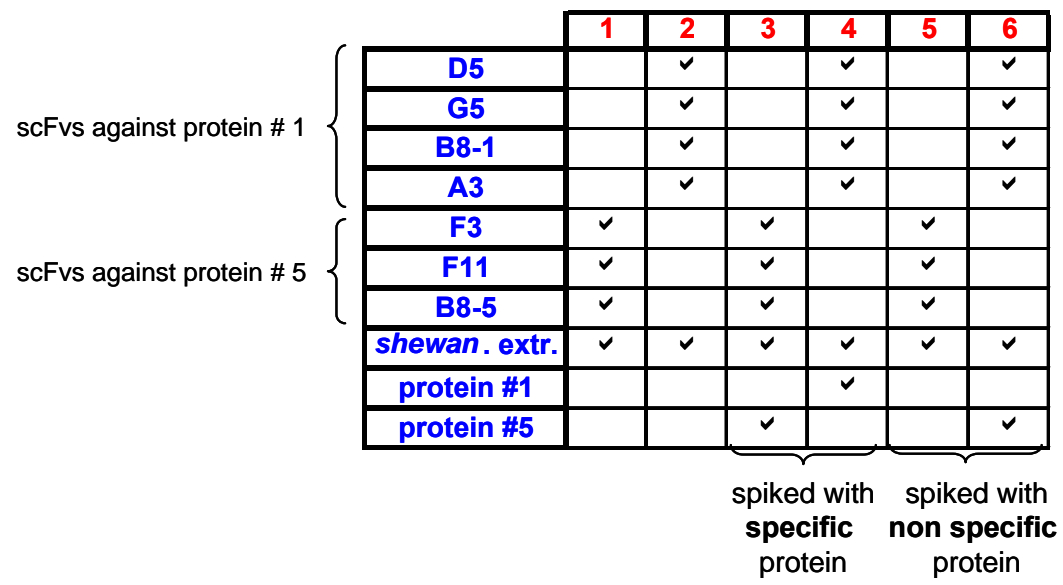

Table 5-1. Components added in each of the I.P. experiments. Approximately 0.7 $\mu \mathrm{g}$ of each scFv was used and $1 \mathrm{ml}$ of $\mathrm{S}$. oneidensis $(1 \mathrm{mg} / \mathrm{ml})$ 
The proteins recovered in samples 1 through 4 were analyzed by Coomassie blue and silver staining. In contrast, the proteins produced in samples 5 and 6 were analyzed by silver staining and Western Blot. The results are shown in Figure 5-6 A-D.

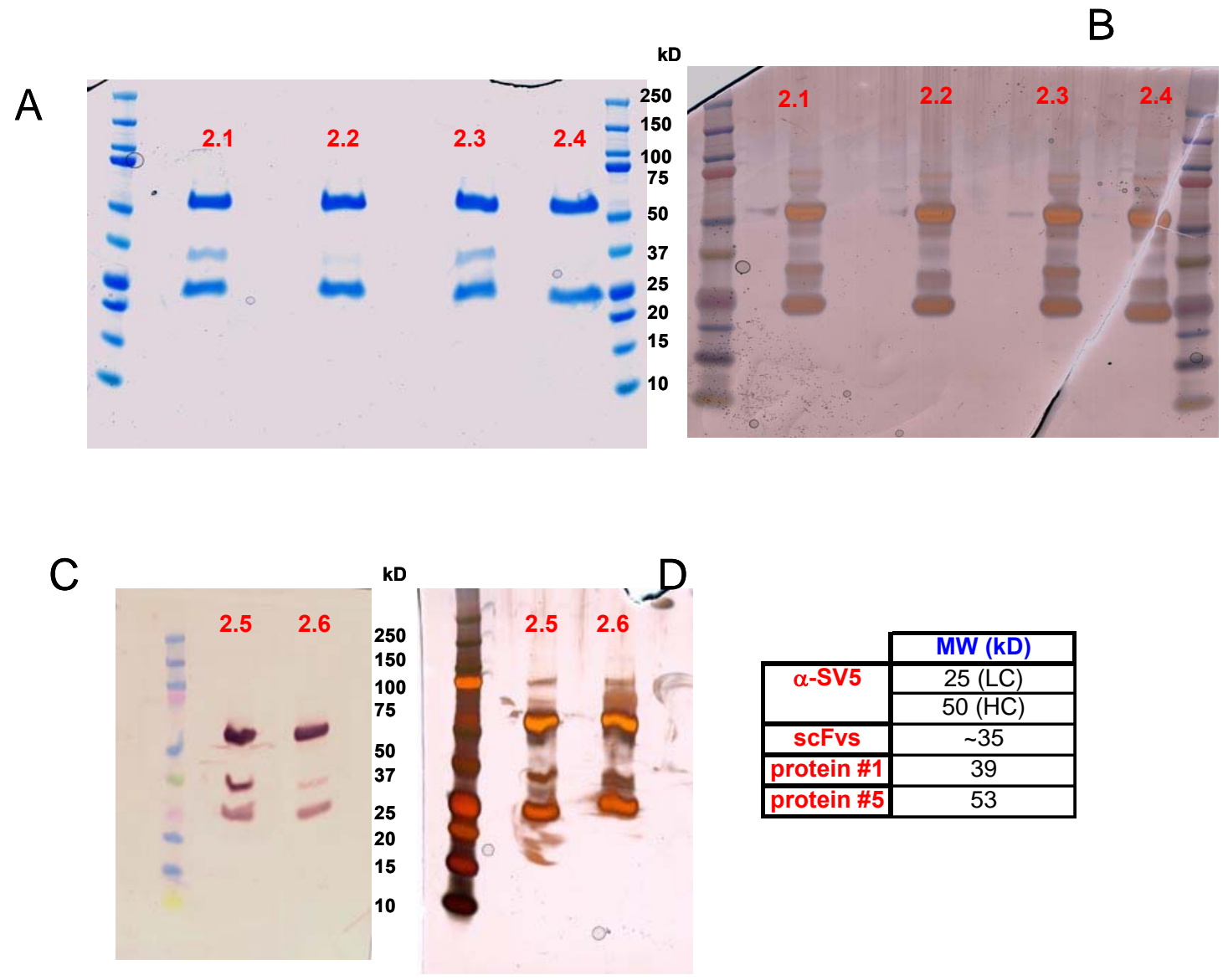

Figure 5-6: Analysis of the Elutions produced in I.P. experiment \#2. Figures 6A and 6B correspond to samples 1-4 analyzed by SDS PAGE and stained with either Coomassie Blue or Silver respectively. Samples 3 and 4 are those spiked with the corresponding target protein for each scFv set. Figure 6C and 6D shows analysis of samples 5 and 6 by Western Blot and silver staining respectively. Samples 5 and 6 were spiked with the corresponding non-target protein for each scFv set. 
The SDS PAGE and silver staining results confirm the presence of the Heavy and Light chains of the $\alpha$-SV5 antibody in all samples 1-6, as expected (refer to Figure 5-6A, 5.6B and 5.6D). The additional protein at approximately $35 \mathrm{kD}$ represents $\mathrm{scFv}$ protein and is visible in all 6 samples when analyzed by SDS PAGE and Silver Staining. Western Blot analysis (refer to Figure 5.6C) confirmed that the scFv proteins from samples 5 and 6 were captured and purified during the IP process. In contrast analysis of the spiked, positive control, samples 3 and 4 do not show recovery of either target antigen \#1 or \#5 at the expected size, $39 \mathrm{kD}$ and $53 \mathrm{kD}$ respectively.

\subsection{Discussion}

Initially a proof of principle experiment was designed to investigate whether single or double tag purification would be advantageous for recovery of IP complexes. The experiment included single or double tag purification of a pre-determined antibody/antigen complex that was created using two different scFvs, D1.3 and HyHel10, which are known to recognize different epitopes of the chicken lysozyme antigen ${ }^{9}$.

The proof of principle experiment was created by mixing the strep-D1.3-SV5 fusion protein with chicken lysozyme and HyHel10-AP. The IP complex was created to demonstrate that all three components of the IP complex can be purified, from E.coli cell extract, using the tag purification system.

Single tag purification was tested using either strep tag II or SV5, fused to D1.3. Results show that Strep Tag II purification successfully captured the IP complex and gave a clean product; where only the bands of the two single chains (strep-D1.3-SV5 and HyHel10-AP) and chicken lysozyme were visible. Single tag purification using SV5 was also successful but gave a less clean product, as the heavy and light chains of the $\alpha$-SV5 
antibody were recovered in addition to strep-D1.3-SV5, HyHel10-AP, chicken lysozyme. The $\alpha$-SV5 antibody heavy and light chains were also eluted because the strong interaction between the SV5 tag and the anti-SV5 antibody ${ }^{10}$ is disrupted by the harsh elution conditions using acidic buffer and a high concentration of salt.

The results of the proof of principle IP experiment demonstrated that double tag purification was the best method for purifying IP complex. Purification using double tag (with each tag at either end of the scFv) ensured that only complexes formed with the target antigen were captured, as the scFv is full length and not truncated. The results also allowed the order, in which to carry out the double tag purification, to be determined; sequential purification using strep tag II followed by SV5. Following the first tag purification using strep tag II, the IP complex was eluted using mild conditions that allowed the components to remain intact for further purification. In addition, the strep tag II purification was cleaner than the SV5 tag purification. The eluted sample produced in the first tag purification was then subsequently re-purified using SV5 tag. Following purification with the SV5 tag, the IP complex was eluted under more harsh conditions that caused the components of the IP complex to dissociate. This was not an issue at this point of the experiment as the final eluted proteins were analyzed under denaturing conditions (SDS PAGE analysis).

The volume of the eluted sample, in addition to purity, was also an important factor in determining the order of the double tag purification. Single purification using strep tag II produced a clean product that was eluted into a very large volume, which then required a second purification that recovered the IP complex in a much smaller sample volume. The combination of the double tag purification provided a very clean product 
(consisting of the 3 IP complex components in addition to the heavy and light chain of anti $\alpha$-SV5 antibody) in a very small final volume. It was necessary to elute into a small volume so the concentration of the IP components would be higher, allowing more accurate identification of the components by mass spectrometry. When analyzing a 'real' IP complex it would be necessary to further identify the individual components of the IP complex using mass spectrometry

After the IP complex had been captured in the proof of principle experiment, the double tag purification system (strep tag II and SV5 tag) was applied to try to immunoprecipitate complexes in a real system using S. oneidensis cell extract. Multiple (30 and 35) different scFvs were previously selected against two different S. oneidensis biotinylated proteins using phage display (Refer to chapter 4). Seven and six scFvs specific for antigen $\# 1$ and $\# 5$ respectively were cloned into pRosa8-strep vector (Refer to chapter 3) to allow each $\mathrm{scFv}$ to be expressed with both strep tag II (N terminus) and SV5 tag (C terminus).

Seven scFv clones originally selected against antigen \#1 and \#5, were expressed and the proteins were subsequently extracted from the periplasm and purified using $\mathrm{Ni}$ NTA agarose beads in two different formats, in column and in tips. Both approaches gave product clean enough to be able to estimate the amount of $\mathrm{scFv}$ extracted. The Ni-NTA purification protocol was not optimized to obtain very pure $\mathrm{scFv}$ protein preparations because the proof of principle experiment required each protein to be spiked into cell extract that contained an array of different proteins. Column format proved to be more suitable for this experiment because it allowed larger volumes of extract to be handled. 
The $\mathrm{scFv}$ purification was performed to concentrate the proteins for quantification so that the amount of scFv spiked into the cell extract could be accurately determined.

S. oneidensis bacterial cells were cultured and the cells were lyzed by homogenization. The amount of protein in the whole cell extract was quantified using the Bradford assay.

In the first experiment, two different sets of scFvs were prepared according to their target antigen; protein \#1 (A3, D5, F3, G5,) or protein \#5 (B8-5, F3, F11). Each $\mathrm{scFv}$ set comprised a number of different $\mathrm{scFv}$ clones that were pooled for the purpose of having a mixture of monoclonal antibodies that could theoretically bind with polyclonal characteristics.

Each scFv set was incubated with a large volume of S. oneidensis cell extract (10 $\mathrm{ml}$ of 1 $\mathrm{mg} / \mathrm{ml}$ ) for two hours at $4^{\circ} \mathrm{C}$ and then with StrepTactin beads overnight. Following double tag purification, SDS PAGE analysis (Refer to Figure 5-5) revealed the presence of two proteins of $50 \mathrm{kDa}$ and $25 \mathrm{kDa}$ that represented the respective Heavy and Light chain of the $\alpha$-SV5 antibody ( $\mathrm{scFv}$ protein) used to capture the IP complex. However, further SDS PAGE and Western blot analysis confirmed that no scFv proteins were recovered following purification. It is likely that no scFv protein was recovered because the proteins were degraded by naturally occurring proteases in the S. oneidensis cell extract. By repeating the experiment with one tenth volume of cell extract and by reducing the incubation time to $1 \mathrm{hr}$, the protease activity could be significantly reduced. Experiment \#2 was repeated with the necessary modifications to prevent degradation of the $\mathrm{scFv}$ proteins. 
In the second experiment the volume of cell extract was reduced to $1 \mathrm{ml}$ and the StrepTactin bead incubation time was decreased to one hour. Four additional control samples were included in the experiment; the cell extract was spiked with target or nontarget antigen for each scFv set being analyzed. These samples were included as controls to confirm the specific interaction of the scFv with target antigen and demonstrate antigen capture

SDS PAGE and silver staining (Figure 5.6A, 5.6B and 5.6D) of the eluted samples showed that all 6 contained two proteins of approximately $50 \mathrm{kDa}$ and $25 \mathrm{kDa}$, that correspond to the respective Heavy and Light chains of the $\alpha$-SV5 antibody used to capture the IP complex. An additional protein at approximately $35 \mathrm{kDa}$ representing $\mathrm{scFv}$ protein was also observed and confirmed by Western Blot analysis (Refer to Figure 5.6C). These results confirm that the scFv proteins were captured and purified from all 6 samples, during the IP process. This result demonstrates that the experimental changes had a positive effect on reducing protease activity and allowing the $\mathrm{scFv}$ proteins to be captured during the double tag purification process.

Despite the success in recovering scFv protein from the cell extract, the results of the 'spiked' positive control samples 3 and 4 (Figure 5.6A and 5.6B) show that the scFvs were not recovered in complex with either target antigen $\# 1$ or $\# 5$ at the expected size, $39 \mathrm{kDa}$ and 53kDa respectively. Silver staining (refer to Figure 5.6B and 5.6D) did reveal extra protein bands, in addition to those representing the Heavy and Light chains of the $\alpha$-SV5 antibody and the scFv protein. However, the banding did not correlate between samples as the same pattern of bands was observed in samples that were spiked with target antigen as well as those that were not. 
There are several reasons why this IP experiment was not successful in the recovery of protein complexes. It is possible that the $\mathrm{scFv}$ protein does not bind to the non-biotinylated form of the target antigen. Originally, the $\mathrm{scFv}$ proteins were selected against biotinylated antigen. The biotin molecule, could in theory, be an important residue at the binding site. However, the biotin molecule is small $(0.2 \mathrm{kD})$ and it is very unlikely its presence or absence would have such a significant impact on the binding interaction between the scFv and biotinylated antigen.

It is possible that target antigen proteins have been captured and recovered at levels that are beyond detection by SDS PAGE and silver stain analysis. If this is the case, methods of IP analysis would require further work to investigate more sensitive ways to detect the presence of the captured proteins. It is also possible that these proteins are not expressed under the culture conditions we used in this experiment.

The affinity of the binding interaction between the target antigen and each individual $\mathrm{scFv}$ is unknown at present. Affinity studies were not carried out due to limited availability of target antigen. The stocks of antigen were used for the phagedisplay biopanning and subsequent screening by ELISA, for the identification of positive binding events. ELISA consumes vast amounts of antigen and this is why some screening was carried out using flow cytometry because the antigen consumption is significantly reduced. As a result the antigen resource was limited to certain experiments and was not available for affinity studies. Nevertheless, the affinity of the scFv for the target antigen is an important factor in the generation of capture antibodies. If a $\mathrm{scFv}$ has a very low affinity for the target antigen then it is possible that the antigen was initially captured in 
complex but was not recovered following purification because it was washed away prior to elution.

\subsection{Conclusion:}

Proteins form part of interaction networks in which the interactions are responsible for regulation and function. The knowledge of the interaction between proteins in these networks can help understand the cellular machinery. Although multiple methods to study protein-protein interaction have been developed, only two groups (Gavin et al and Ho et al) have done high throughput studies based on in vivo complexes (as opposed to yeast two hybrid methods) where they have identified unprecedented amounts of interaction information. Both methods are based on the use of a tagged bait protein which is used to pull down a protein complex. Comparison of the results obtained between these two methods and with other methods, where only single protein-protein interaction was studied (e.g. yeast two hybrid), have yielded poor overlap between them. Although this could be explained due to each method studying different properties, it is also known that half of the current high-throughput data are spurious ${ }^{11}$. For this reason there is a need to develop as many complementary methods as possible, to increase the confidence in identified interactions. The method proposed in this thesis involves the use of $\mathrm{scFv}$ antibodies to capture the bait protein and pull down complexes formed with any other proteins. The main advantage of this system is that the bait protein is not genetically modified and remains in its natural conformation. This is an essential factor when investigating complicated protein interactions and elucidating the significance of their biological role. This method also involves the use of a double tag system (strep tag II, SV5 tag) which allows for the double purification of protein complexes. The first 
purification step is done under physiological conditions allowing the complex to remain intact and undergo a second purification step. In the second purification, the high affinity of the tag (SV5) towards the antibody in the matrix allows the recovery of probably most of the complex, while the format used (Phynexus tips) allows the elution in a very small volume which is ideal for later analysis. Despite the problems faced in these studies, double tag purification using $\mathrm{scFv}$ proteins continues to be a feasible method for the capture of IP complexes, and the work described in this thesis provides the experimental framework and underpinning to further these studies. Although additional analytical experiments will be required to investigate the factors required for successful capture and recovery of IP complexes using this system, we believe the basic structure of the approach has been defined. Further studies will be needed to investigate the interaction between the selected scFvs and purified Shewanella proteins, and more sensitive analytical techniques will have to be applied for the detection and identification of IP complex components, including LC-MS/MS. 


\subsection{References}

${ }^{1}$ Sblattero, D.; Bradbury, A., Exploiting recombination in single bacteria to make large phage antibody libraries. Nat Biotechnol 2000, 18, (1), 75-80.

${ }^{2}$ Ayriss, J.; Woods, T.; Bradbury, A.; Pavlik, P., High-throughput screening of single-chain antibodies using multiplexed flow cytometry. J Proteome Res 2007, 6, (3), 1072-82.

${ }^{3}$ Lupas, A., Coiled coils: new structures and new functions. Trends Biochem Sci 1996, 21, (10), 375-82.

${ }^{4}$ Tripet, B.; Yu, L.; Bautista, D. L.; Wong, W. Y.; Irvin, R. T.; Hodges, R. S., Engineering a de novo-designed coiled-coil heterodimerization domain off the rapid detection, purification and characterization of recombinantly expressed peptides and proteins. Protein Eng 1996, 9, (11), 1029-42.

${ }^{5}$ Chao, H.; Bautista, D. L.; Litowski, J.; Irvin, R. T.; Hodges, R. S., Use of a heterodimeric coiled-coil system for biosensor application and affinity purification. J Chromatogr B Biomed Sci Appl 1998, 715, (1), 307-29.

${ }^{6}$ Pavlik et al., in preparation

${ }^{7}$ Pavlik et al., in preparation

${ }^{8}$ Butler, J. E.; Ni, L.; Nessler, R.; Joshi, K. S.; Suter, M.; Rosenberg, B.; Chang, J.; Brown, W. R.; Cantarero, L. A., The physical and functional behavior of capture antibodies adsorbed on polystyrene. J Immunol Methods 1992, 150, (12), 77-90.

${ }^{9}$ Zhou, H. X., Quantitative account of the enhanced affinity of two linked scFvs specific for different epitopes on the same antigen. J Mol Biol 2003, 329, (1), 1-8.

${ }^{10}$ Hanke, T.; Randall, R. E., Variable domain sequences of $m A b$ with high affinity for a linear oligopeptide. Immunogenetics 1995, 42, (5), 442-3.

${ }^{11}$ Fields, S.; Song, O., A novel genetic system to detect protein-protein interactions. Nature 1989, 340, (6230), 245-6. 


\section{Curriculum Vitae:}

\section{Maria Rosa Valero Aracama}

rosuka@gmail.com

439 Connie Av.

Los Alamos, NM, 87544

Tel: (505) 672-2293

\section{Education:}

West Virginia University, USA

Ph.D. in Chemisty, December 2007

University of Alicante, Spain

B.S. in general Chemistry, December 1996

$\underline{\text { Research Experience: }}$

July- September 1994: Analytical Chemistry research at the Institut Für Mechanische Verfahrenstechnik und Mechanik, University of Karlsruhe, Germany.

July- September 1995: Analytical Chemistry research at the Department of Mineral Resources at the Imperial College of Science, Technology and Medicine, London, United Kingdom.

June 1999-January 2001: Analytical Chemistry, focused in mass spectrometry research at the Department of Chemistry at West Virginia University, W.V, USA.

February 2001- July 2007: Molecular Biology research for PhD degree at Los Alamos National Laboratory, Los Alamos, NM, USA

Honors/Awards:

July 1994: International Association of Student Exchange (IAESTE) scholarship (Germany)

July 1995: International Association of Student Exchange (IAESTE) scholarship (United Kingdom)

Teaching experience:

1998-1999: Teaching assistant in undergraduate physical chemistry laboratories

1999-2000 Teaching assistant in undergraduate general chemistry laboratories

Publications:

Ayriss, J. V., R.; Bradbury, A.; Pavlik, P., Multiplexed Flow Cytometry. HighThroughput Screening of Single-Chain Antibodies. Humana Press: 2007; Vol. Therapeutic Antibodies.(in press) 
Velappan, N.; Martinez, J. S.; Valero, R.; Chasteen, L.; Ponce, L.; Bondu-Hawkins, V.; Kelly, C.; Pavlik, P.; Hjelle, B.; Bradbury, A. R., Selection and characterization of scFv antibodies against the Sin Nombre hantavirus nucleocapsid protein. J Immunol Methods 2007, 321, (1-2), 60-9.

Kiss, C.; Fisher, H.; Pesavento, E.; Dai, M.; Valero, R.; Ovecka, M.; Nolan, R.; Phipps, M. L.; Velappan, N.; Chasteen, L.; Martinez, J. S.; Waldo, G. S.; Pavlik, P.; Bradbury, A. R., Antibody binding loop insertions as diversity elements. Nucleic Acids Res 2006, 34, (19), e132.

\section{Poster Presentations:}

Rosa Valero, Leslie Chasteen, Jennifer Martinez, Peter Pavlik, Nileena Velappan, Andrew Bradbury (2003). Development of functional tag-scFvs fusions, Antibody Engineering, San Diego, CA

\section{Memberships:}

October 1999-currently: Member of The Phi Lambda Upsilon Honorary Society. 